

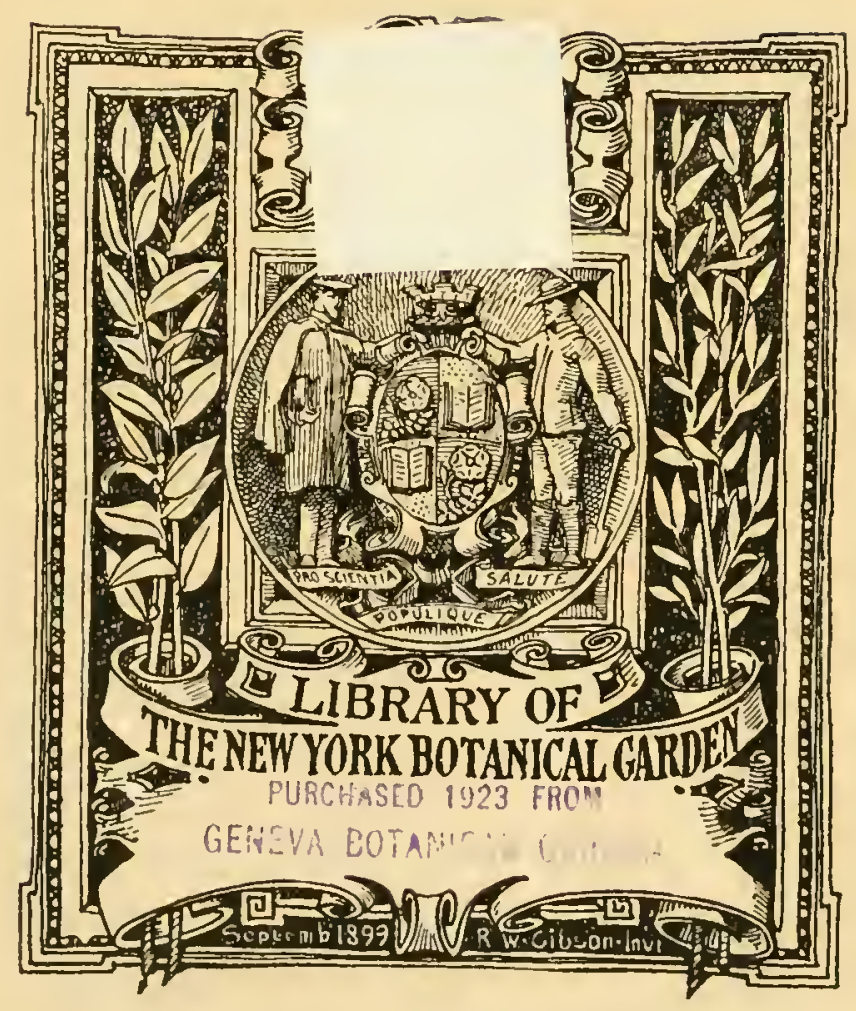


DUPLICATA DE LA BIBLIOTHÊQUE DU CONSERVATOIRE BOTANIQUE DE GENEVI VENDU EN 1922 

v. Halácsy.

\section{BOTANISCHE ERGEBNISSE}

EINER IM AUFTRAGE DER HOHEN KAISERL. AKADEMIE DER WISSENSCHAFTEN UNTERNOMMENEN FORSCHUNGSREISE

IN GRIECHENLAND.

\section{BEITRAG ZUR FLORA VUN EPIRUS}

VON

\section{Dr. EUGEN v. HALÁCSY.}

(9Kit 3 Tafdn.)

BESONDERS ABGEDRUCKT AUS DEM LXI. BANDE DER DENKSCHRIFTEN DER MATHEMATISCH-NATURIVISSENSCHA FTLICHEX CLASSE DER KAISERLICHEN ARADEMIE DER WISSENSCHAFTEN.

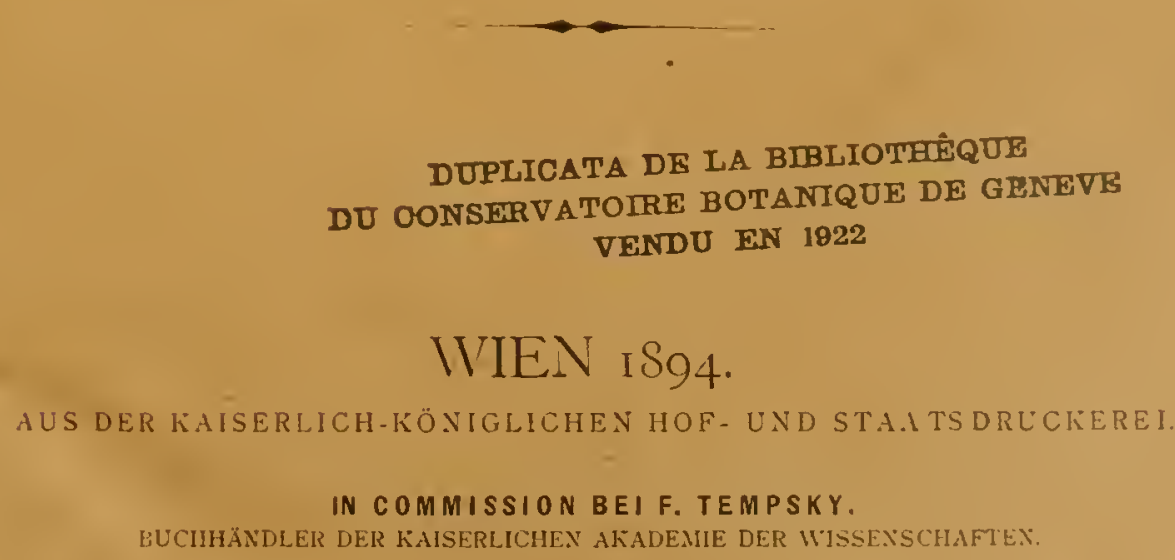

Preis: I fl. $75 \mathrm{kr} .=3 \mathrm{Mk} .50 \mathrm{Pf}$. 



\title{
BOTANISCHE ERGEBNISSE
}

EINER IM AUFTRAGHE DER HOHEN KAISERL AKADEMIE DER WISSENSCHAFTEN UNTERNOMMENEN FORSCHUNGSREISS

\author{
IN GRIECIIENLAND.
}

\section{BEITRAG ZUR FLORA VON EPIRUS}

VON

\section{Dr. EUGEN v. HALÁCSY.}

\author{
(Q) it 3 Jafelu.)
}

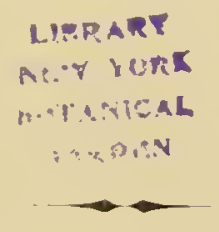

\author{
WIEN IS94.


. 429 


\title{
BOTANISCHE ERGEBNISSE
}

EINER IM AUFTRAGE DER HOHEN KAISERL. AKADEMIE DER WISSENSCHAFTEN UNTERNOMMENEN FORSCHUNGSPEISE

\author{
IN GRIECHENLANI). \\ I. BEITRAG ZUR FLORA V(ON EIPRUS \\ YON \\ Dr. EUGEN v. HA LÁCSY. \\ (OVit 3 Jafin.)

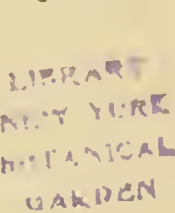

VURGFLEGT IN DER SITZUNG IOM 8. FFBRLAR 189.1

\section{Allgemeine Übersicht.}

Mit dem Namen Epirus wurie ursprünglich die den nördlichen innischen lnseln gegenüberliegende Küste des Festiandes bezeichnet. Erst in späterer Zeit wurde dieser Name auf jene Landschalt des alten Heilas beschrïnkt, welche im Norden durch die acroceraunischen Gebirge (Ergenik, Tsannusi und Mitsikeli), im Westen durch das Jonische Neer, im Süden durch den Golf ron Arta und im Osten durch Thessallicn, bezichungsweise die Pinduskette begrenzt wird.

Seit dem Mittelalter stand das ganze Land unter türkischer Herrschaft und erst in neuester \%eit wurde ein im Osten gelegener, durch den Fluss Arachthos begrenzter schmaler Strich desselben an Griechenland abgetreten und hiedurch die Möglichkeit geboten, bei den in jeder Richtung hin civilisirteren Verhältnissen der griechischen Lïnder, gegenüber den unter türkischer Ferrschaft belindlichen Prorinzen, in denselben behufs wissenschaltlicher Forschungen Reisen zu unternehmen.

Die Vegetationsverhältnisse dieses griechischen Antheils, die Eparchien Arta und Tsumerka umfassend, sind es auch allein, als die im heurigen Sommer durchforschten, die im Folgenden bespruchen IIerden sollen.

Bezüglich seiner Flora gehört Epirus zu den am wenigsten untersuchten Läindern der Palkinhalbinsel: der zu Griechenland gehörige Antheil Wurde bislang überhaupt ron keinem Floristen betreten.

Die ersten horistischen Angaben über das Land finden sich in A. Boué, La T'urquie d'Europe (Paris 18to) enthalten und bestehen in der Aufzihlung einer Anzahl Pflanzenarten, ohne nähere Angabe des Fundortes. Eine zweite Arbeit in den Sitzungsherichten des hotanischen V'ereins der Provinz Brandenhurg, $\therefore \mathrm{N}$, im Jahre 1879 publicirt, hat den für die griechische Flora hocherdienten Furscher Th. V. Heldreich zum Verfasser und besteht aus einem V'erzeichnisse der ron N. K. Chodzes bei Restoration, einem östlich ron Argyrokastron gelegenen Orte, im Sommer 1878 gesammelter Pllanzen. Es werden daselbst b8. zwejtelsohne den unteren Regionen angehöriger Arten aufgezählt. 
Mit diesen beiden Publicationen erscheint die Literatur üher dic Flora des Epirus erschöprt. A. BalJacci, ein Bolngneser Botaniker, hat zwar den nördlich won Prevesa gelegenen Berg Zalonges bestiegen und von demsethen, wie auch von seiner Küstenausbeute Exsiceaten vertheilt, jedoch noch nichts weiteres üher seine Untersuchungen veröfentlicht. Auch lassen jene, soweit diesulben eben bekannt sind, keine genaueren Sehlüsse ïher die Vegetationsterhältnisse des Landes zu.

Dass die V'egetation von Epirus eine reiche und mannigfaltige sein muss, ist schon aus der Lage des Landes am Jonischen Mcere einerseits, wie auch antererseits seiner orographischen Verhältnisse Wegen anzunehmen. Ex analogia mit den benachbarten Ländern wird an ler Küste die ron Quarnero bis zur Siilspitze des Peloponnes verbreitete Mediterrantlora ihre Verbreitung linden, und es wäre von hohem Interesse, festzustellen, wicweit sich dieselbe in das lnncre des Landes erstreckt. Andererseits ist wieder a priori schon anzunchmen, dass die mächtigen Kalkgebirge des Landes, deren höchste Gipfel üher $2000 \mathrm{~m}$ ('Tsumerka $2330 \mathrm{~m}$. Strungula $2018 \mathrm{~m}$, Peristeri'2290 m) messen, cine Hochgebirgsliora heherbergen werden, welche vermöge der Unterlage und ler Nachbarschaft jener der bekannten Hochgehirge Griechenlands ähnlich sein muss. In dieser Richtung hin wäle wieder dic Frage zu lösen, ob und eventucll wic weit andere Florenelemente vom Norden her etwa in diese eindringen.

IV'enn auch zur Aufliärung dieser Verhältunisse ein einmaliger hüchtiger, nur auf cinige Wochen ausgedehnter Besuch sicherlich ungenügend ist und leineswegs hinreicht, um eine vollständige Charaliteristik der Vegetation zu geben, so bietet derselbe doch Anhaltspunkte in hinreichender Zahl, um über dic pllanzengeographischen Verhältnisse des Landes wenigstens einigermassen sich orientiren zu können.

Der griechische Antheil von Epirus ist ein Gebirgsland in strengstem Sinne, welches nach Sülen $2 \mathbf{u}$ terrassenartig in den Golf ron Arta abfillt und durch den im Norden des Landes entspringenden, in den cbengenannten Goll mündenden Arachthos und den erst bei Missolounghi in das lonische Meer sich ergiessenden Acheloos durchströmt wird. An der Mündung des ersten Fiusses breitet sich eine Niederung - Potamia - aus, welche der Hauptmasse nach Meerstrandspflanzen enthalten dürfte. Diesc Nielerung bildet auch zugleich dic Eingangspforte der mediterranen Flora, welche entlang des Arachthos-Thales, allerdings immer mehr und mehr Einbusse erleidend, nördlich bis gegen Vulgarelion hin ihre Repräsentanten sendet und die ganze Ostküste des amhracischen Golfes behertscht.

Wie für die übrigen Länder des mediterranen Becliens, sind auch hier das Charaliteristische die immergrünen Buschwälder wder Macchien. Sie betecken als undurchdringliche Dickichte mit geringen Unterbrechungen die Vorberge der ganzen Ostküste des ambracischen Golfes von Karvassaras in Acarnanien, bis südlich von Arta, ferner nördlich von dieser Stadt sämmtliche Vorberge der Tsumerka-Kette bis oherhalb des borfes lialentini. Sie werden zusammengesetzt durch Pistacia Lontiscus, Myrtus communis,

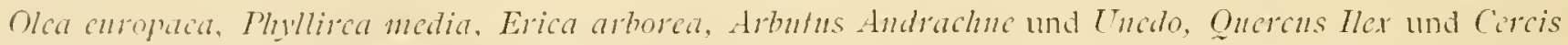
Siliquastrum. Spartium juncem und Juniperus phocnica, welche in anderen Gegenden oft einen Piestandtheil dieser Macchien bilden, Wurden hier nicht beobachtet. Der den Sonnenstrahlen unzugängliche Grund dieser Dickichte wird von Statulen und Kräutern zumeist gemieden, dagegen lindet sich in Lichtungen und an den Ründern derseiben eine reichliche Vegetation vor. Von den Arten, die diese zusammensetzen, wären als mehr minder tonangebende zu erwähnen: Clematis Flammula, Delplinium

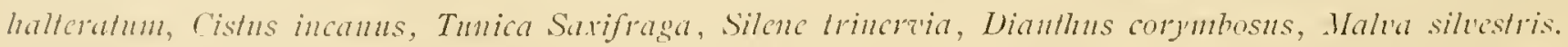
Palimms anstrulis (\#uweiten kleine selbständige Bestände biliend), Rluns Cotinns, Ononis antiqunmm,

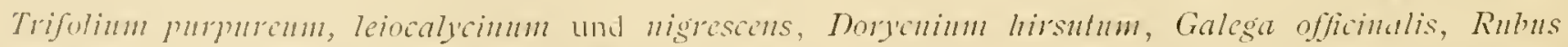

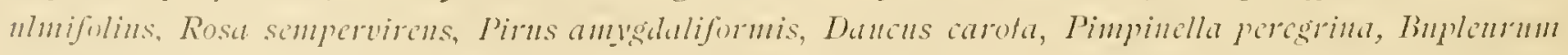
scmidiathanmm, Erynginm campestre und creticum, Lonicera drusca, Callistemma brachiatum, Scabiosa

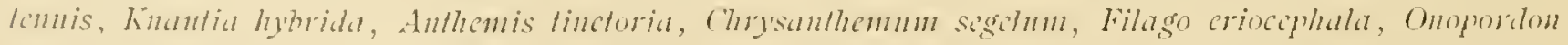

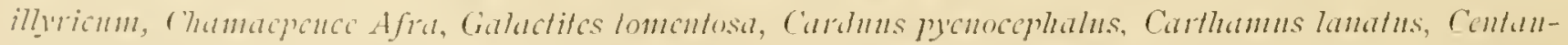

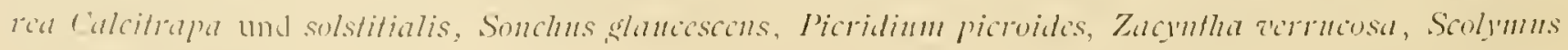




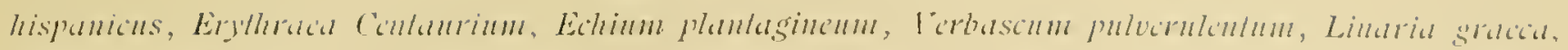

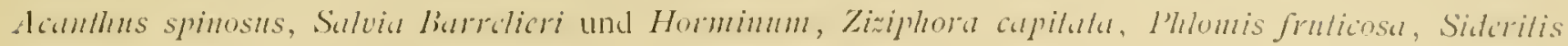

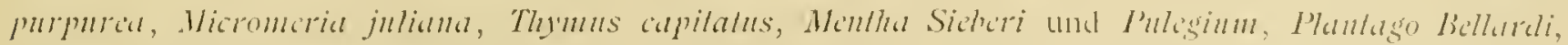

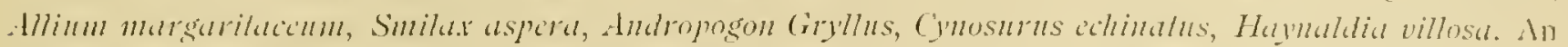

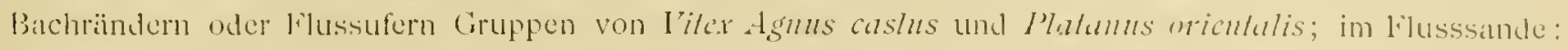

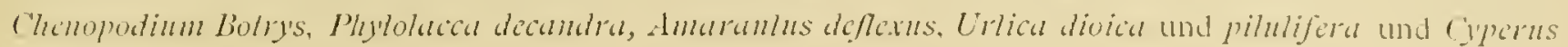
lingsus.

Wie Weit in das Innere des Landes nach Osten zu diese ehen geschilderte Macchienformation vordringt, lionnte nicht festgestellt Werjen; in ler unmittelbaren Umgebung ler Stalt Arta findet sie jeduch eine Hnterhechung und macht einer Staudenvegetation Platz, deren fast alleinigrer liepräsentant Phlomis frulicosa hildet, welche Art in unzählbarer Menge die dic Stadt behersehenden Höhen mit ihren zahlreichen grossen Blüthen gelb färbt, im verblühten Zustande jedoch dem überwucherten Landstriche durch das gratue Culorit ein unfreumaliches Ansehen gewährt und nuch dem Floristen der in ihr vorlindlichen atussesprochen armseligen Vegetation wegen Wenig lnteresse bictet. Palinrus aculallus, Onoporion illyricum,

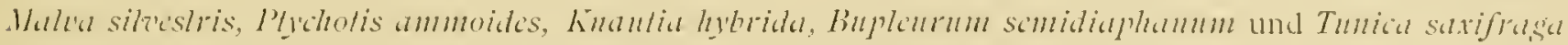
sind fist ausschliesslich dic in diesej Phlomis-lilu. Unterlinft findenden Arten.

Unweit nördlich von Arta entfaltet sich jedoch von Neuem die Macehienvegetation und bedeckt sämmtliche etwal 300- $400 \mathrm{~m}$ hohen Vorberge mit ihrem dichten Gebïsche. Zu den ohen aufgezählten Arten treten hier noch Callecolome villosa, Colmea arborescens und Pteris apulima; letztere stellenweise tunangebend. Mehr minder ansehnliche Mais- und Kornfelder, dann auch unmittełbar um Arta einige Öbaumplantigen machen nur zum kleinsten Theile Concurenz dieser ursprünglichen Vegetation.

Bei kalentimi, etwa $15 \mathrm{~km}$ nördlich von Arta, geht die Vegetation in eincr Seehöhe von 350 - $400 \mathrm{ml}$ in jenc der Bergregion über. Successive bildet sich aus dem immergrünen Buschwalal cin durch hohe Stimme grobildeter Mischwakl aus. Myrthe. Pistacie, Ölbaum und Eriøa arbora verschwinden und nur

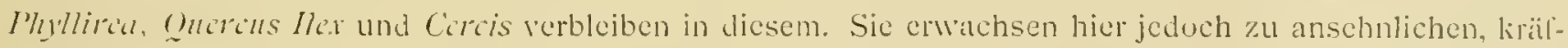

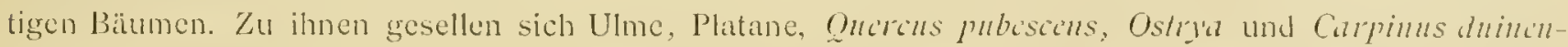

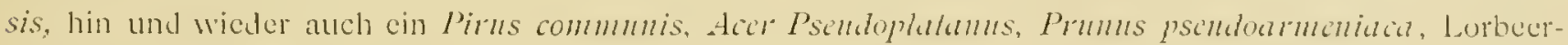
haum viler Fraxims crèlsior. Diese genannten Batmarten bilden ausgedehnte, theilweise recht schattige Wäilder, welche auf ihrem Untergrunde die günstigsten Verhältnisse zur Entwicklung einer reichen Krïuter- und Staudenvegetation schaffen. Zum Theil bestebt diese aus Arten, welche schon zur Biluluner Jer Macchienflora beitrugen, zum Theil aber aus neuen Elementen, wie: Nigalla damascina, Dolphininm

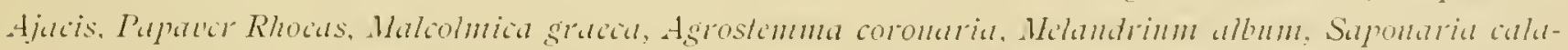

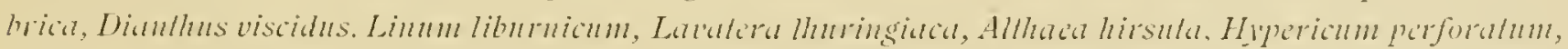

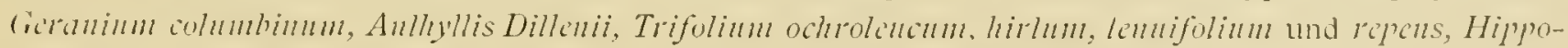

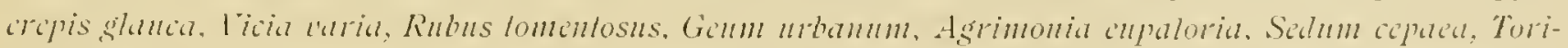

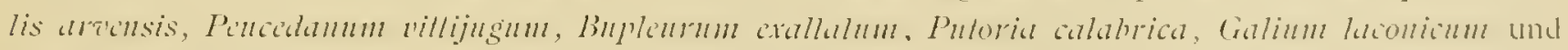

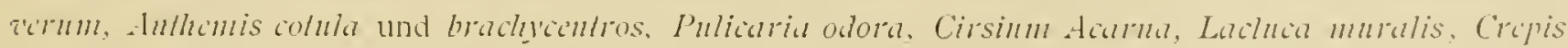

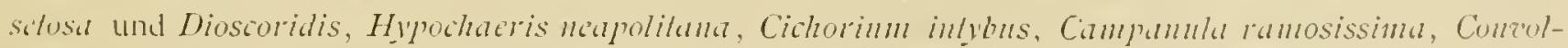

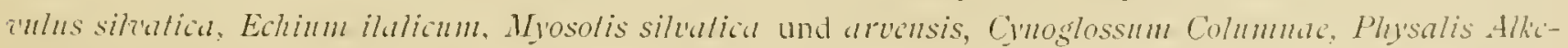

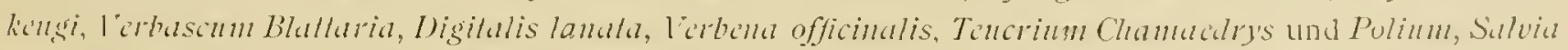

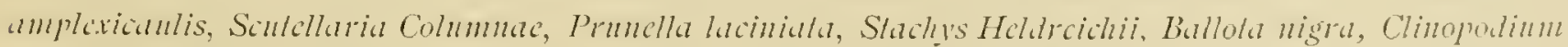

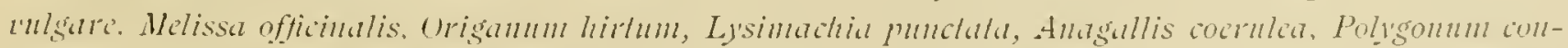

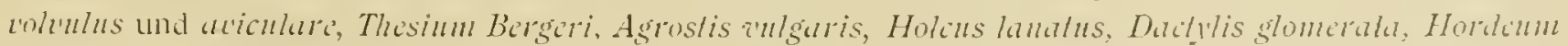
bubesmm und mmrinmm; von Farnen: Celcrach. Pleris aquilina, Adiamtum, Asplenimm Trichomanes und lirgilii, Aspridim Filix mas, nebst einer Mlenge von Rindenflechten und Mloosen. Clematis ritalha und litis silvestris schlingen sich lianenatig bis in die Wipfel der Bäume, während andere Stätmme wicder von Epheu umwuchert erscheinen. Vereinzelt tritt auch Cormus mas und Juniprrus o.jycilms its Unterholz all. W゙leinere oder grüssere llaldwesen sind mit kurzem schütteren Grase bewachsen und beher- 


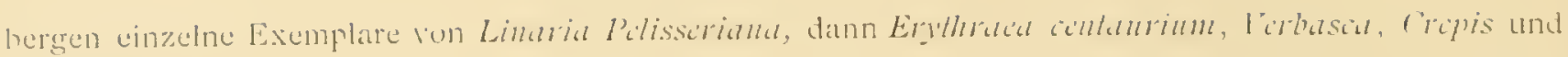
Anthc mis-arten.

In einer Sechühe ron 7.50-900 m macht der Mischwald der dritten Region, jener der ApolloTatnne Platz. Letztere bildet hier mehr minder schüttere Bestünde, umgibt gleichsam als ein Gïrtel das (ichirge etra bis zur Höhe von $1500 \mathrm{~m}$ und greift zungenfömig in cinzulnen Sehluchten und an Abhängen in die wberste Region hinauf. Auf dem üstlichen Abhange des Tsumerka, wherhalb Vulgarelion, Wurde die where Grenze mit $[390 \mathrm{~m}$, an den östlichen Ahfällen des Peristeri mit $1,500 \mathrm{~m}$ und an dem südlichen Ahhange des letzteren bei Kalarrytae mit $1140 \mathrm{~m}$ festgestellt.

Wenn schon in der Bergreugion die Repräsentanten der Mediterranflora sich allmählich an Arten- und Individuenzahl verminderten, so verlieren sie sich in der Tannenregion bis auf einige Arten fast günzlich.

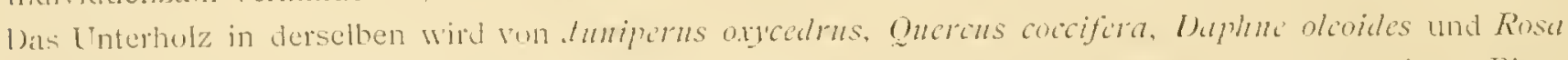

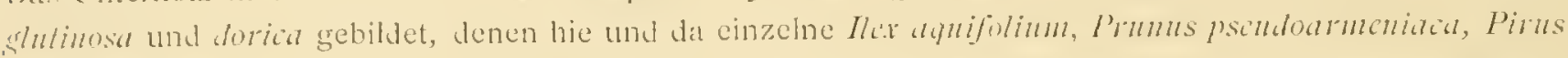
"ambislaliformis und an Gebirgshächen Sumbucus chulus, Salix incalua, purpurad und die Platane, welehe hier bis zu einer Sechöhe von $1200 \mathrm{~m}$ hinaufsteigt, beigemischt erscheinen. Tonangubend in derselben ist wicder der Adleffarn, weleher stellenweise ganze Abhänge dicht bekleidet. Von Stauden und Krïutern sind in ihr, wie in der Bergregion, vorwiegend die Elemente dreier Floren rertreten, der balkanischgrichischen, der baltisch-mitteleuropäischen und der mediterranen. Häufigere Reprïsentanten der ersteren

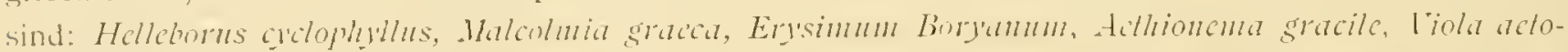

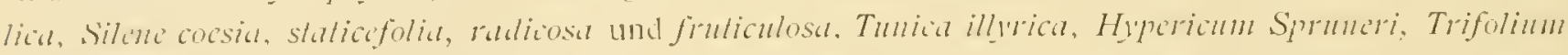

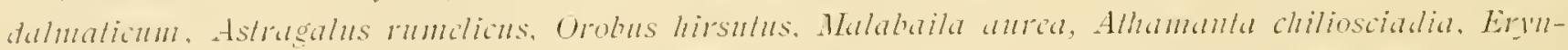

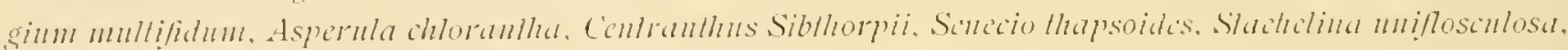

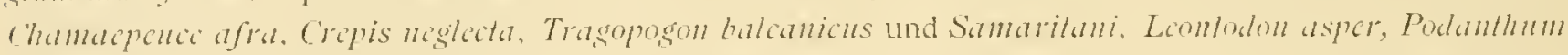

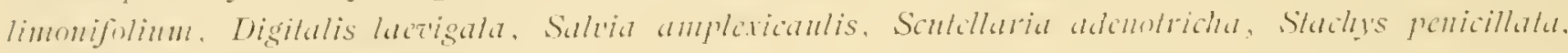

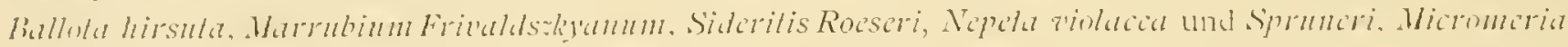

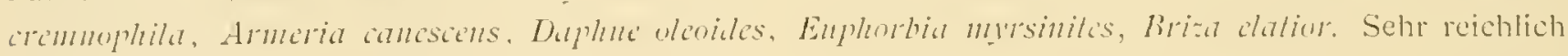
vertreten in der Tannenregion sind die Elemente der baltisch-mitteleuropäischen Frora. Es sind die

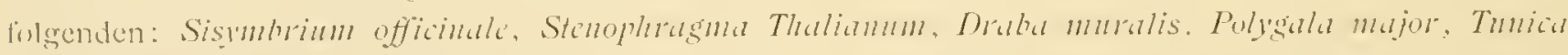

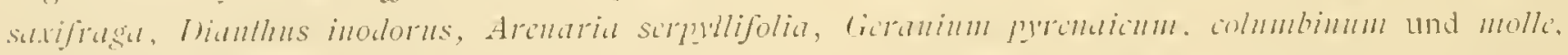

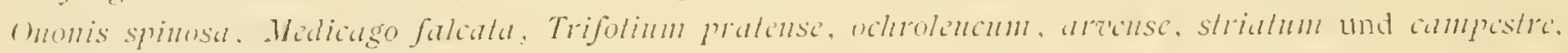

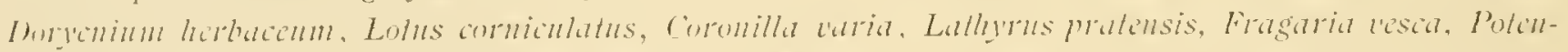

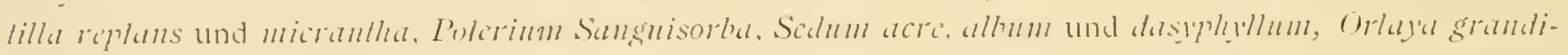

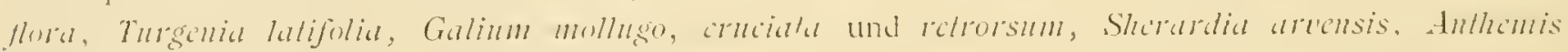

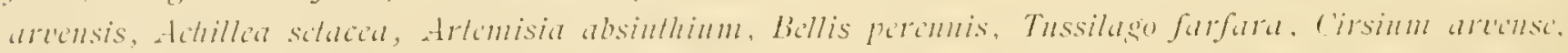

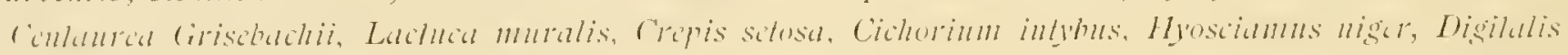

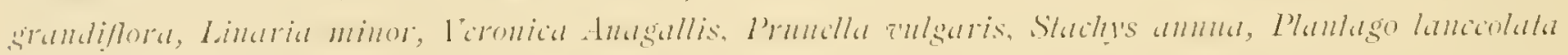

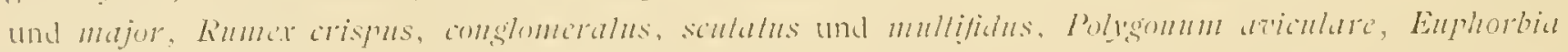

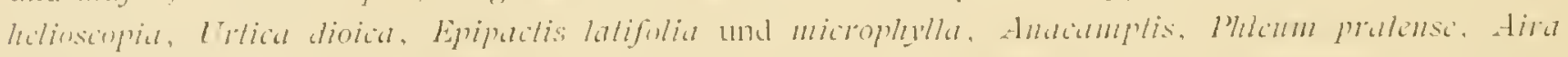

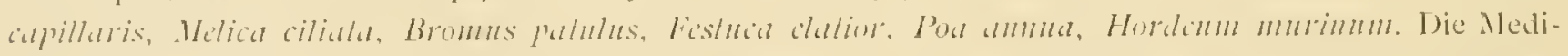

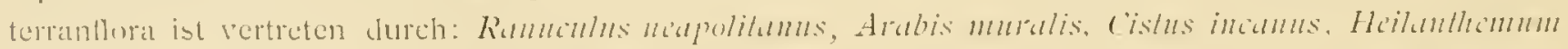

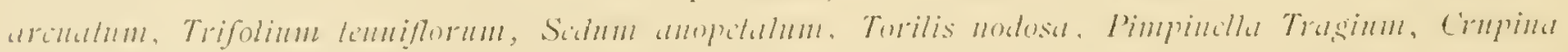

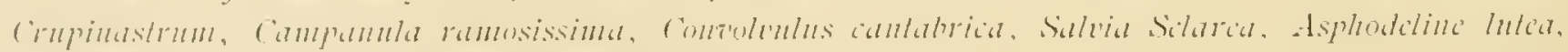

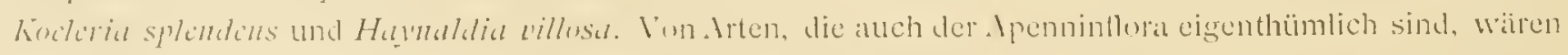

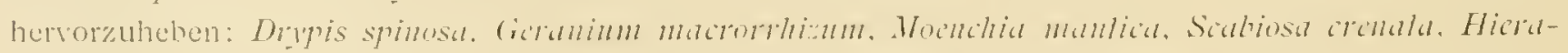

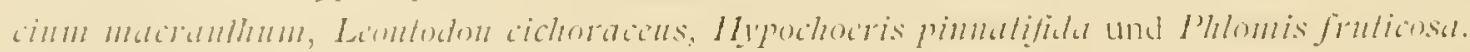

In der wheren Grenze der Tannenregion wird noch hin und wieder Mais cultivit, allerdings in litummerlichen spamnenlangen Individuen.

IVihrend die Tannenregion einer zusammenhängenden Grasnarle fast bölibg enthehrt, ist die auf sie lingende vollig batumberse obere Region oft auf weite Strecken hin von Ciramatten beleckt, welehe der 


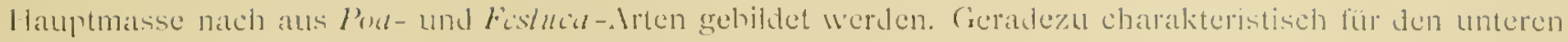
und mitferen Theil dieser keggion ist das stellenweise massenhafe Aultreten vom . Allerfarne, von

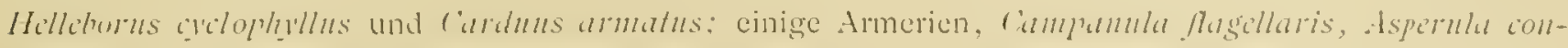

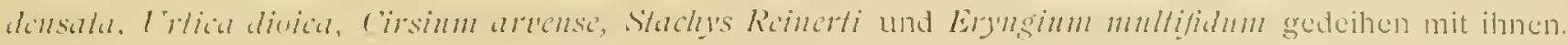

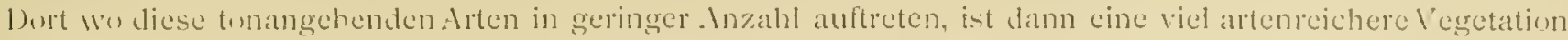

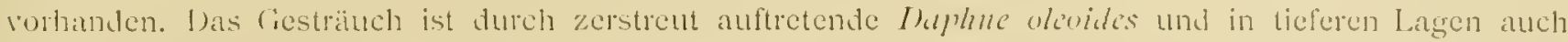

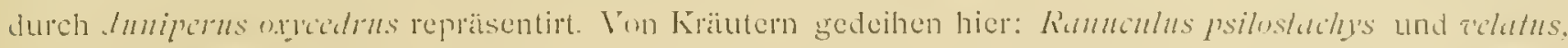

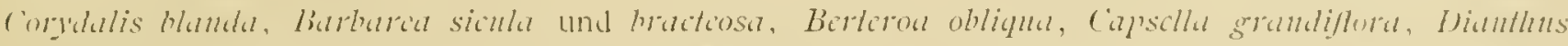

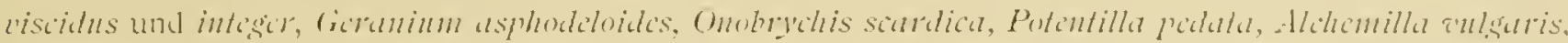

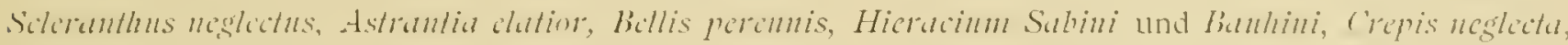

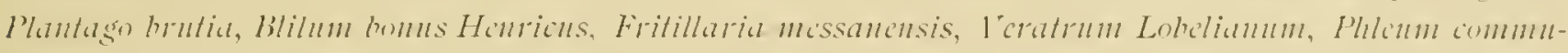

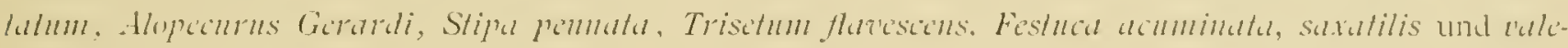

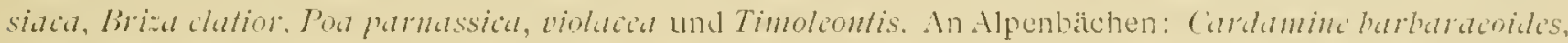

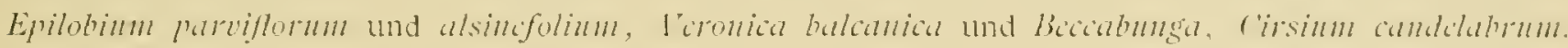
Sehr mannigfaltig ist die Felsenfiora und jene des Felsenschuttes; sie wird dureh folgende, grösstentheifs der griechischen Hochgehirgsflora angehörige Arten zusammengesetzt: Rammmculus comcinmalms und

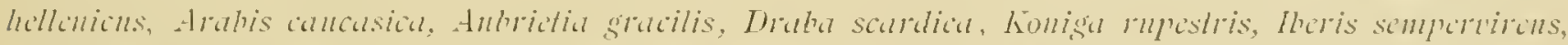

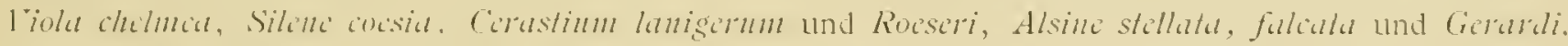

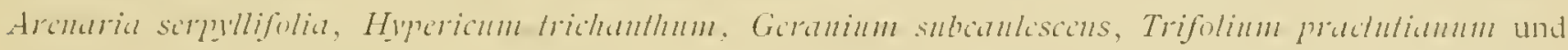

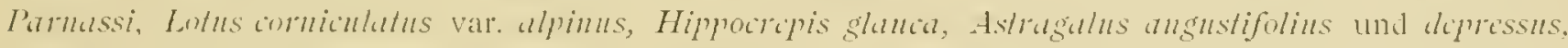

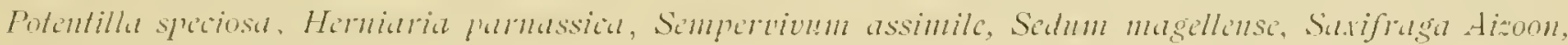

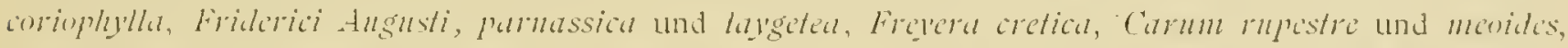

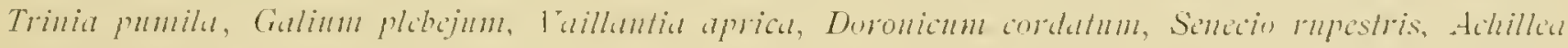

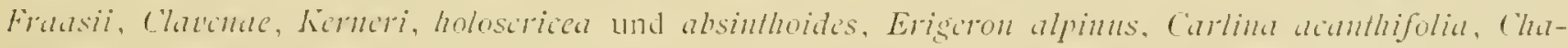

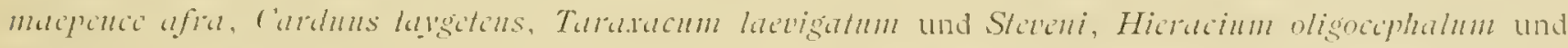

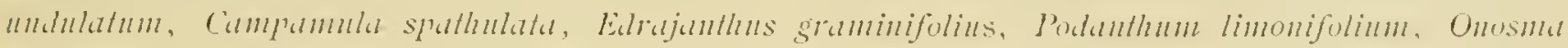

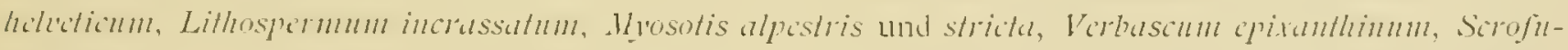

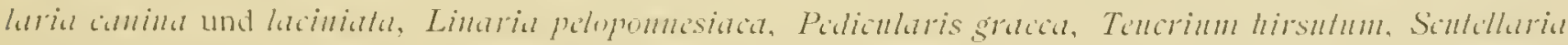

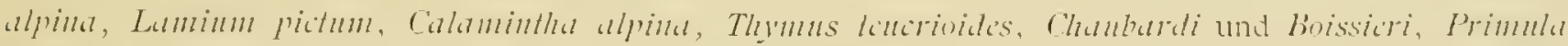

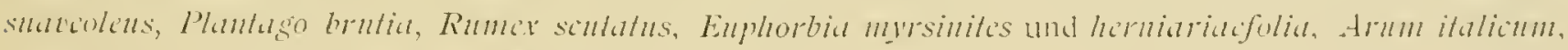

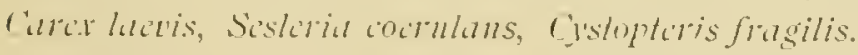

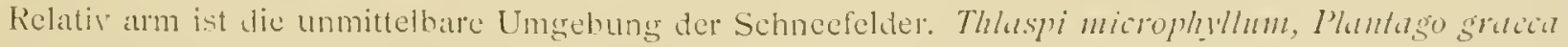
frocus l'behonsis und Scilla miblis sind fast allein die daselbst vorkummenden Arten.

Alpenweiden. Apentosen, rentianen und Soldanellen fehten auf dem epirotischen Huchgebirge gainzlich.

Aus den ehen seschilderten Vegetationswerhältnissen des ästlichen Epirus geht heror, dass diesus Gebirgsland in seiner Vegetation hezügrlich der Pflanzenregronen die grössten Analogien mit den griechischen Gehirgen atulweist. Hier wie dort lassen sich in verticaler lichohtung vier Regronen deutlich unterscheiden und zwar 1. die mediterlane, 2. dic Berg-, 3. Jic Tannen- und endlich t. dic HochgebirgsRegion. Aus den orürterten Verhältnissen greht ferner zur Evidenz hervor, dass die Hauptmáseo der Arten der epirntischen Gehirge, insbesondere jene der Hochgehirgsregion dieselhe ist, die auch in den griechischen Gebirgen heimisch ist. und dass wiewohl schon eine ziemlich hetrïhtliche Zilhl von Arten dul mitteleuropäisch-haltischen Flora - jedentalls eine grössere als in den südlichen griechischen Gebirgen in Epirus angretroffen wurde, die Flura des Landes doch noch entschieden zur griechisch-mediterranen zu zählen ist. Das allmähliche Verschwinten diverser südlicher, und das cbenso allmählicho Auftauchen einzelner nördlicherer 'lypen ist ührigens schon in diesem relativ kleinen Gebicte eine auffillige Erscheinung;

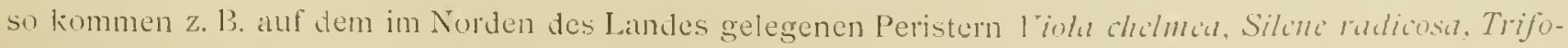

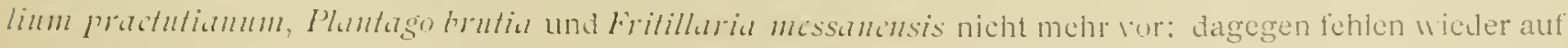




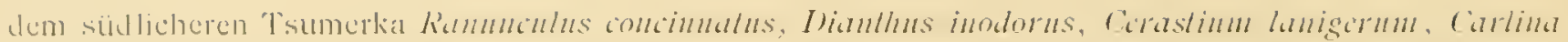

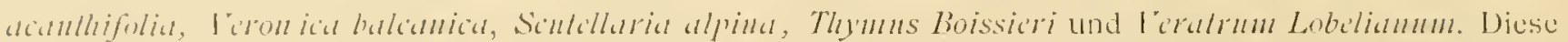
Erecheinungen, inshesondere in Mitberücksichtigung älndicher Vorkommnisse in den benachbaten macedunisch-albanischen (iebirgen, machen es böchst wahrscheinlich, dass eine stricte (irenze beider erwähnten Florengebiete katum vorhanden sein dürfte, Jass vichmehr beide liloren successite in einander iibergehen.

\section{Itinerarium.}

Am 1. Juli 1893 verliess ich Athen und fuhr mit meinem Reischegleiter Carl Sehwalrzenherger. Buchdruclereihesitzer in Wien, dem Natulaliensammler Christos Leonis in Athen und mit ron der griechischen Regierung zu unserem Schutze mitgegehenen zchn Mann Infanterie mit Herin Lieutenant Platon Chry santhopoulos an der Spitze, mit der nordpeloponnesischen Bahn nach Patras. De! 2. duli wurde zur Ausrïstung Jer Expedition verwendet. Am 3 . Juli ïbersetzten wir den Golf von Patras und fubren mit der actolischen Bahn von kironeri nach der Endstation Agrinion, wo wir Mittags ankamen. Der Nachmitag desselben 'Tages wurde noch zu einem Ausfluge in die Umgebung verwentet. Am l. Juli luhr ich nach kravassaras in Acamanien und machte Aufsammlungen in der Umgebung dieses Ortes. Am ¡. Juli untersuchte ich die Macchien an der Ostlï̈ste des Golfes von Arta, kam Mittags desselben Tages in Arta selbst an, hotanisinte in der Umgebung auf den benachbarten llügeln und im Arachthos-'Thale. luzwischen traf Her lieutenant Chrysanthopoulos seine Anordnungen zur Herbeischaffung der zur leise im Gebirge nothwendigen 15 Maulthiere. Am 6 . Juli erfolgte der Aufbruch der Faravane; auf SaumWegen arreichten wir nicht ohne Nühe die Ortschaft Kalentini, in deren Nähe das Nachtlager unter freiem llimmel bezogen wurde. Am 7. Juli wurde die Bergregion zwischen Kalentini und der sehon an ler unteren Gränze Jer Tannenregrion licgenden Ortschaft Vulgarelion untersucht. An S. Juli besticg ich zum erstenmale den Tsumelia, gelangte Abends nach Theodoriana und unternahm von hier aus den näichsten Tag den zweiten Aufstieg bis auf die Spitze (23:36 m). Den 10. Juli bestieg ich den Strungula (20) s $m$ ), gieng denselben Tag noch nach Melisurgi hinab und gelangte am 11. Juli über l'ramanta und Mazuki an den Ahähgen des Rakardista vorbei in das Dorl Kalarytac, in welehem Orte ich bis 1t. Juli verblieh, Jie Zeit zu Excursionen in der Imgebung verwendend. Fon hier aus wurde am 1t. Juli Jer l'eristeri

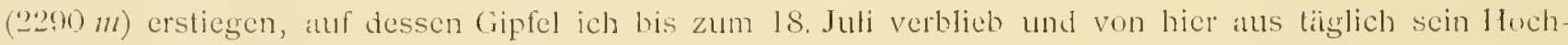
pateatu in verschiedenen Richtungen durchstreifte. Am 19. Juli stieg ich nach Chaliki hinab und gुelangte nich Thessalien, in welchem Lande ich nur kurze Zeit mehr zubringen konnte, da ich mit dem an "26. Iuli von Pirateus abfahrenden Lloyd-1)ampler die Heimreise anzutreten grezwngen war.

Ls erübrigt mir hier nur noch meinen ehrfurchtsiollsten Dank abzustatten vor Allem der hohen kaserlichen Akademic der WVissenschalten, welche durch Gewährung einer bedeutenden Subvention meine Forschungrspeise ermöglichte und in zweiter Linie Herrn Hofrath Prolessor Anton Kerner v. Marilaum, birector des botanischen Gartens der k. k. Universität in Wrien, auf dessen Initialive diese keise zu Stande kim und dessen lreundliche Rathschläge und liräftiger Beistand, nach jeder likehtungr hin, für mich von unschatzbarem Werthe waren. Zu ganz besonderem Danke fühle ich mich ferner verpllichtet gegenüher Seiner Execlen\% dem Minister des Imneren Herrn Olivier Marquis de Bacyuehem, auf

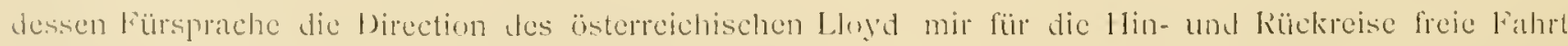

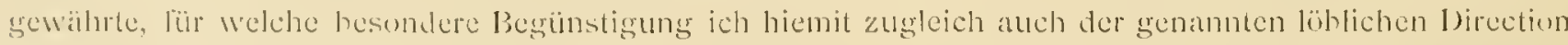
meinen höflichsten Dink abzustatten mir erlathe. Mcinen ergebenen Dank sage ich weiters der lüblichen

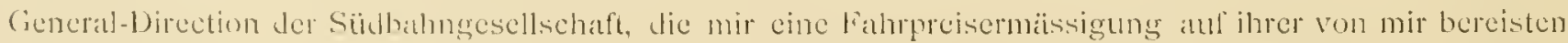
Strecke bewilliegt hatte; fernel tem Leiter Jer gendätischen Ahtheilung des k. k. Militaireographisehen Institutes in Wien, Ilemen (J)erstieutenant Ileinrich Hartl, unter dessen Sehut\% und thatliriftigem Beistand ich die sonst kaum auslührbare Bereisung des Epirus volliuhren konnte; dem lieutenant der

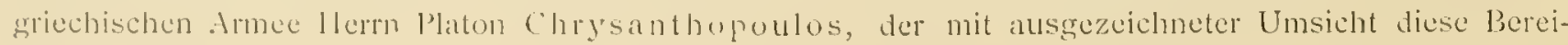


sung leitete; endlich meinem liehen freunde Theolor v. Heldreich, Director des botanischen Gartens in Athen, Jessen kathschläge Wesentlich fördernd mein Unternehmen unterstützten und llerm Custos

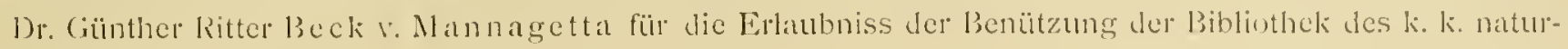
historischen llofmuseums.

\section{Aufzählung der auf dieser Reise in Epirus beobachteten und zum grössten Theile auch gesammelten Pflanzenarten.}

Die Reihenfolge der Arten ist jene in C. F. Nyman Conspectus Florac Europaene. Als Arten wrurden jednch zum Theil auch Formen aufgezählt, welche im genannten Werke als sogenannte Subspecies oder Varietäten aulgelührt crscheinen. Es wurde hiebei dem Grundsatze gefolgt, dass Pllanzenformen, Welche sich durch constante, wenn auch nicht besonders markante Merkmate von ihren nächsten Verwandten unterscheiden und in Menge aultreten, auch als Arten aufgefasst wurden. Eine Ahstufung in Subspecies, als einer jüngeren einer Art entstammter Form, dieses Mitteldings zwischen Art und Varietït, wurde hiedurch vermieden, schon aus dem Grunde, weil in den meisten Fällen der Nachweis jener Abstammung nur sehr schwer klargestellt werden kann.

Entsprechend den ron einem grossen Theile der Fachmänner acceptirten Priorititsgesetzen wude jede Art mit ihrem ältesten Namen angeführt, sei dieser ihr als Species in der glcichen oder in ciner anderen Gattung, oder als Varietät vom Autor gegeben worden. Ich halte dieses Vorgehen für len einzig richtigen Weg, um zu einer einheitlichen Nomenclatur zu gelangen und kann anders gearteten Anschauungen über Prioritätsprincipien aus bekannten, hier nicht weiter zu erörternden Gründen nicht heipflichten. Dic Citate sind fast durchwegs den Orginalpublicationen entnommen, eine Arbeit, die zwar nicht ohne erheblichen Zeitauliwand ausgeführt werden konnte, die mir jedoch schon aus dem Grunde nothwendig schien, da in den floristischen Werken dieselben nicht selten unrichtig sind. Die seichen Biblintheken Wiens, wie jene des k. k. Hofes, des k. k. naturhistorischen Hofmuseums und des botanischen Mluseums der k. k. Universität, crmöglichten es auf dicse oft schwer erhältlichen Quellen zurückzugreî́en.

In der Schreibweise der Standortsangaben folgte ich der vom k. k. militärgengraphischen lnstitute in Wien (1855) herausgegebenen Generalkarte des Königreiches Griechenland im Masse 1:310000. Es wurden hiebei genau die Region und die Neereshöhe, in welcher die Aiten beobachtet wurden, wie auch olt die Art und Hïurigkeit des Vorkommens derselben angegeben.

\section{RANUNCULACEAE Juss.}

1. Clematis flammula L. Sp. pl. p. 544 (1753).

In Gebüschen der Mediterran-Region bei Kalentini, sclten. Höhe $100 \mathrm{~m}$.

2. Clematis vitalba L. Sp. pl. p. $544(1753)$.

In Cebüschen der Bergregion bei Kalentini; in der Tannenregion des Strungula oberhalb Melisurgi und des Peristeri bei Mazuki. Zerstreut. Höhe 500-800 $\mathrm{m}$.

3. Ranunculus psilostachys Griseb. Spicileg. Fl. Rum. et Bithyn. I, p. 304 (1843).

Auf steinigen Grasplätzen in der oberen Region des Peristeri, einzełı. Höhe $1500 \mathrm{~m}$.

Die lixemplare rom Peristeri stimmen sowohl 'mit der Diagnose Griscbach's a. a. O. als auch mit dem Originalexemplare, Welches ich im Herbare des $k$. k. Hofmuseums einzuschen Gelegenheit hatte, übercin. R. psilostachy's steht zweifelios dem westlichen R. monspeliacus L. zunächst, unterscheidet sich jałoch von ihn durch die dichten seidenharigen Blättes, die stark keiligen Blattabschnitte, den meist reicher veriistelten Stengel und den nicht hakig eingekrümmten Schnabel der Früchtehen. Letzteres Unterscheidungsmerkmal ist allerdings nicht durchgreifend, da einzelne mir vorliegende serbische Exemplate des R. psiloslachly's, bisweilen ebenfalls hackige Schnäbel aufreisen. Hauptsïchlich auf dieses Merkmal hin glaubte auch Petrovic die Nišcr Pflanze als cine noue Art auffassen zu müssen und 
heschrieb sie in keinen Adlit. ad Flor. agri Nyssani p. 21 unter dem Namen R. Missamus. Man lindet jedoch selbst unter seinen (originalexemplaren welehe mit nicht hakigen Schnëbehn, und es ist daher vollständig gerechtfertigt, wio os auch Velenorsky in seiner Flora hulgetrica gethan, die Petrovic'sche Art als Synonym zu R. pisinstachl's zu stellen, umsomehr, als die weiteren von P'etropic angegehenen, die Biatter hetreffenden Charaktere unconstant und hauptsïchlich nur bei üppig entwickelten Exemplaren rorhanden sind. Magerere Exemplare haben ganz und gar die Plätter des gewöhntichen R. psilostacly's.

Jläufig findet man R. psilostachls mit dem ehenfalls die Balkanhalbinsel bewohnenden, zu den grumosen kanunkeln mit herahgeschlagenen kelchnipfeln gehörigen R. rmmclicus Griscb. verwechselt, trotzlem Grisehach letzteren ganz treffend durch dic kurzen rundlichen Wrurzelknollen, den nur 1-2 blüthigen stengel, die dreilappigen (bei $R$. psilustachl's dreitheiligen) Blätter und dic nicht seidige Belileidung der Blattunterseite rom ersteren unterscheidet. Urheber dieser Verwechshungen scheint Jank al zu sein, welcher in seinen Bemerkungen zu Boissier's Flora orientalis in öst. bot. Zeitschr. XX. p. 11 die beiden erwïhnten Arten besprechend, die von Boissier für R. mmelicns gehaltene Planze aus Attical für R. psilostacly's erkiürt, weil der seidenhanrige Überzug der Blätter, welchen Janka als cinzig annehmhares Unterscheidungsmerkmal gelten lässt, indem er die übrigen von Grisebach und auch ron Buissier hervorgehobenen Charaktere für keine durchgreifenden hält, jener nicht abgeleugnet werden kann. Gerade diese Merkmale an den Wurzelknolken und der Blattheilung sind aber an den von Janka a. a. O. citirten Exemplaren Heldreich's und Orphanides' thatsächlich vorhanden und so deutlich ausgesprochen, Jass Grisebach sie zweifellos als zu seinem R. mumelicns gehörige crklärt hätte; ja sellst dic Beharung ist keineswegs jene seidenhaarig glänzende, welche cben $R$. psilostacly's aufweist. Ich kann daher die Anschauung Janka's, welcher sich übrigens auch Boissicr im Suppl. Flor. or., f. 30 nachträglich irrigerweise accommedirt hat, nicht theilen und halte die von Boissier anfïnglich ganz richtig gedeutete Pflanze vom Parnes und Pentelicon für R. munclicus. Zu diesem gehört auch übrigens dic Pflanze vom Nit. Elins nuf Thasos, welche von mir in üst. bot. Zeitschr. XLll, p. +12 fälschlich als R. psilushachy's publicirt und von Sintenis sub Nir. 482 vertheilt wurde.

Von R. monspliacus J., dem derselbe durch die Bekleidung und die Amblüthigkcit (obzwar letzteres Merkmal nicht durchgreifend ist) noch näher steht, als der $R$. psilostaclz's, ist er durch die kurzen 11 urzelknollen und dic aufrechtabstchenden nicht hakigen Schnähel zu unterscheiden.

Nachstehonde Tabclic soll die Unterschicde der drei besprochenen Arten veranschaulichen:

\section{R. monspliacus L.}

Wurzclknollen länglich-cylindrisch.

Stengel 1 - wenighlüthig, kurz anlicgend behart.

blïtter anliegend kur\%harig, die grundständigen dreilappig oser Jreitheilis, Arschnitte villiahel.

Früchtehen halkig-geschnähelt.

\section{R. rnmelicus Griscb.}

Wurzelknollen kurz - ellipsoidisch.

Stengel 1 - wenigblüthig, kurz anliegend behaart.

Blätter anliegend kurzharig, die grundständigen dreilappig, Abschnitte breiteiömig - keilig (relativ kürzer und breiter).

Früchtchen nicht hakig-greschnäbelt.
R. psiloslacleys Griscb.

W'urzelknollen länglich-cylin drisch.

Stengel mehrblüthig, lang ahsite hend behart.

Blätter seidenhaarig-glïnzend, die grundständigen dreitheilig, Abschnitte keilig (relativ länger und schmailer).

Früchtehen meist nicht hakig-weschnïbelt.

Was die Verhreitung der heiden letzten Arten anhelangt, so scheint R. rmmelions auf Jen ïstlichen Theil Jer Ballianhalhinsel ron Constantinopel bis Attica beschränkt zul sein. Ich sah ihn aus: Thracien, in agge Byzantino leg. Noe, Nr. 32 sub R. flulellate: Constantionopel, leg. Noe, Nr. 204 sub R. monspeliaco: prope Kalofer, leg. Janka; Macedunien, Saloniki, leg. Friedrichsthal in Herh. Maced. Nr. 1034. (.Vle im Herhare Jes Wiener Iofmuseums). lnsel Thasus, Mt. Elias, leg. Sintenis und Bornü̈ller in Iter

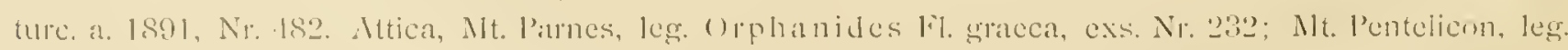
Helateich llerh. grace. norm.. Nr. 6ri. 
K. psitostachys scheint dagegen melu die westliche liälfe der Balkanhalbinsel und den Peloponnes zu berrohnen. Ich sah ihn aus: Serbien, bei Niš und V'ranja, leg. P'etrovic, als R. missumus. Albanien: 1tt. Peklen prope lpek, leg. Firiedrichsthal llerb. Maced., Nr. 323 (Wiener Hofmuscum), bei Zeleniku, leg. Dörfler a. 1803. Rumelien, leg. Frivalds\%ky Nr, 110 (Wiener Holmuscum). Bulgarien: Drendem Tepe bei Philippopel, leg. Sintenis und Bornmüller in 1ter ture a. 1891, Nr. 10. Giricehenland, in regione abietina Mt. 'laygetns, leg. Heldreich. lch sclbst sammelte ihn endlich auf dem epirotischen Peristeri und auf dem Panachaicon in Achaia.

4. Ranunculus velatus. Spec. nora. (Sectio Euranunculus (iren. et Godr. Fl. Fr. 1, p. 29). Perennis viridis; rhizomate obliquo ad collum fibris erectis velatn; caule crecto, $25-40 \mathrm{~cm}$ alto, sparsin piluso, monantho vel supra medium Innge ramoso bifloro: folis sparsim pilosis vel subglabris, basilaribus vagina brevi ciliata instructis, $3-4$ conformibus longissime petiolatis, caule triente brevioribus, ambitu late cllipticis 3-5 partitis, segmentıs distantibus cuneato-obovatis acute incisis, caulinis 1-3 amplexicaulihus sessilibus digitatis, laciniis oblongo-cuneatis trisectis vel lineari-lanceolatis et tunc subintegris integrisve; floribus aureis, diametro $22 \mathrm{~mm}$ latis; pedunculis longis teretibus, adpresse pilosis, superne nudis; calycc luten, sepalis patentibus oblongo-oratis obtusiusculis, extus albo-rillosis; spica ellipsoidea vel subglohosa, staminum toro pilis nullis nbsito, receptaculo apice parce piloso; carpellis obovato-rotundatis, carinatomarginatis nec bisulcatis, glabris, minutissime punctatis, in rostrum validum recurvum uncinatum eis triplo brevius abeuntibus.

Hahitat in herbidis humidiusculis regionis superioris Nt. Tsumerka supra pagum Theodoriana. Alt. $1600 \mathrm{~m}$. Solo calcareo.

Ich hätte die an und für sich ansehnliche Zahl der habituell mehr weniger sehr ähnlichen, einen behaarten Fruchthoden besitzenden Arten der Section Euranmuculus, nicht noch um eine vermehrt, wenn es nach genauer Prüfung der einschlägigen Arten mir möglich gewesen wäre, sie in den Formenkreis der cinen oder der anderen Art unterzubringen. Dieselhe brachte das Resultat, dass die ehen heschriehene Art ron ihren nïchsten Verwandten durch bestimmte Merkmale abweicht, welche im Folgenden des Nïheren erörtert Werden sollen. Yon den Arten der genannten Gruppe und zwar in dieser wialer von jener Untergruppe, welche gekielte und neben dem Fiele nicht gefurchte Früchtchen hesitzen, sind es romehmlich R. I'illarsii DC. und R. montanus Willd., welchen $R$. velalus zunächst stcht.

Ersterer, welcher wie Jordan im Schultz' Archiv I, p. 305 machgewiesen, mit $R$. aduncus Giren. et Godr. = R. lapponicus Vill. (non L.) identisch ist, lässt sich durch dic langen Fruchtschnäbel, welche halb so lang oder noch länger als die Frucht sind, ron R. vclalus sofort unterscheiden. Derselbe ist cine dem südlichen Frankreich, dem benachbarten Italien und der südwestlichen Schweiz cigenthümliche drt und scheint nicht weiter nach Osten vorzudringen. Alle Angaben über das Vorkommen derselben in den östlichen Florengcbieten, dürften auf Yerwechslungen mit nahe verwandten Arten beruhen.

Es bleiben also cigentlich nur $R$. monlanus und jene in den Formenkreis dieser Art gehörige Ranunkel zum Vergleich übrig, welche Fruchtschnäbel besitzen, die höchstens $1 / 3$ so lang als dic Frucht sinc. W'as nun $R$. monlanns selbst anbelangt, so ist von diesem die beschriebene Art durch den reichfaserig-schopligen Wurzelstockhals, die tieforgetheilten mit spitzigeren Zipfel versehenen Blätter. die ellipsoidischen (nicht kugeligen) Fruchtköpfchen und die längeren Fruchtschnäbel verschieden. Zumeist ist auch R. montamns viel hleiner. Von $R$. carinlliacus Hoppe unterscheidet sich $R$. relatus durch fast dieselhen . Verkmale und ausserdem durch viel breitere Blattziptel und den hohen Wuchs. Beiden letzteren Arten charakteristisch ist ührigens der reichfaserig-schopfige Wurzelstockhals. Bei Berücksichtigung der eben angeführten Merkmale ist eine Verwechslung des $R$. v'clalus mit diescn beiden Arten wohl ausgeschlossen. Dagegen ist eine solche mit einer anderen, dem $R$. monlanus nahestehenden Art, insbesondere Wenn man die Beschreibung lerselben allein vor sich hat, viel leichter. Diese Art ist der ziemlich in Vergessenhcit gerathene $R$. concinmutus Schott, Östem. botan. Wochenh. VII, p. 182 aus Montencgro. Thatsächlich steht auch R. 2'clatus dicsem am nächsten; durch den aufrechten, viel höheren Wuchs, die bedeutend verlängerten Blüthenstiele, dic 
ellipsoitischen Fruchtkïplehen und den hackigen nicht wie hei jenem eingerollten Schnainel ist er jedoch von jenem auch leicht zu unterscheiden. Auch R. acutilobus l.e d. Fl. Ross. I, p. 40 seheint der Beschreihung nacls mit R. relatus sehr nahe verwandt, jedoch kaum mit ihm identisch zu sein. Exemplare von diesem konnte ich leider nicht zur Einsicht erhalten. Endlich wäre noch R. hrominus ("rantz=R. Hornschnchii Hoppe (rergt. Kerner. Schedac ad Fl. exstirp. Austro-1Fung., Nr.99) zu crwähnen, weleher sich jedoch nehst anderen Merkmalen, schon durch den dicht beharten Torus und das gleichfalls dichtbehiarte Receptaculum ron R. leclutus sich unterscheidet.

5. Ranunculus concinnatus Schott, in öst. hot. Wochenbl. N11, p. 182, (185i).

Im Kalligerölle der höchsten Erhebungen des Peristeri, oft in der Nähe von Schneefeldern truppen"weise. Höhe $2000-2290 \mathrm{~m}$.

Die vorliegenden Exemplare stimmen sowohl mit der Beschreihung Schott's, als auch mit den rom rerstorbenen Hofgüitner Maly am Loween in Montenegro gesammelten Exemplaren, nach welelen der lutor dic Art aufstellte, gut üherein. Vom zunächst verwandten R. montumus Willd. unterscheidet er sich dureh den reichfaserig-schopfigen WVurzelstockhals, die langhanig gewimperten Bhattscheiden der tiefurgespaltenen und mit spitzeren Zipretn versehenen grundständigen Blätter und dureh die längeren fruchtschuähel. Der ihm hahituell ebenfalts nahestehende $R$. Sartoriamus Boiss. und Heldr., weicht ron ihm nelost der viel dichteren Behaarung des unteren Stengeltheiles, der Blattstiele und Blattscheiden, durch die riel schmäleren Blattzipfel ab und verhält sich diesbezüglich etwa zu ihm, wie R. carinthiacus Hoppe zu R. montunns. Durch diesetben Nerkmate unterscheidet sich von ihm auch R. orcophilus M. a. Bieb. 11. Taut. Cauc. 111, p. 383, weteher übrigens sowohl der Diagnose, als auch des allerdings rudimentüren Steven'schen, im Herbare des Wiener Hofmuseums befindlichen Exemplares nach dem R. Sartoriamus sehr nuhe stehen muss, wenn er nicht gar mit diesem identisch ist. Dass ersteres wenigstens der Fall ist. beweist auch die rerschiedene Deutung eines Ranunkels von der kyllene im Peloponnes, Welehen Orphanides, offenbar auf Boissier's Detemination hin, in seiner Flora gracea exsiccata Nr. 231 als R. demissus vertheilte. In den Diagn. pl. or. Ser. Il, Nr. 1, p. S erklïrt ihn Boissier später für R. orcopllitus M. a. Bieb. und endlich in der Flora orientalis I, p. 40 rür R. Fillarsii DC. - Wettstein hält die liyllenePlianze in Beitr. Flor. Alban. p. 15, gestützt auf die Beschreibung Bieberstein's und auf den Vergleich mit kaukasischen Exemplaren für den echten R. orcophilus und betont, dass dieselbe mit der von Dörfler im l.jubitrn in Scardus gesammelten Exemplaren identisch sei, welcher letzteren Ansicht ich mich ührigens vollinhaltlich anschliesse, hichei jedoch erwähnen muss, dass das obenerwïhnte Steven'sche Exemplar allerdings eine bedeutend intensirere Behaarung aller Theile aufweist. Haussknecht endlich hailt in Mittheil. Thüring. hot. Verein 1893, p. 98 dieselhe Pflanze für R'. Surtorianus und zieht zu dieser' Art auch jene vom liarava, welche zum Theil wenigstens nach meiner Anschauung zu R. concimatus grehiort.

Diese rerschiedenen Beurlheilungen ein und derselben Pflanze beweisen also, dass R. orcophilus und R. Sarforiants mindestens sehr nahe stehen müssen. Aus dem Erörterten geht aber auch zugleich herror, dass atuf der Balkanhalhinsel eine Reihe von, dem R. monkanns verwandten Arten, wachsen, welche nur durch relativ geringfügige, hauptsächlich die Beharung und die Blattheilung betreflende Mcrkmale, ron einander sich unterscheiden. So zeichnet sich z. 13. durch schr geringe Behanang und breite Blatlabselnitte l. velutus vem T'sumerkagebirge aus, der übrigens auch durch die Grïsse von den folgenden hahituell abreicht. Hhm schliesst sich $R$. concinnalus aus Montenegro und vom Pindus an, weleher ren nicdrigerem

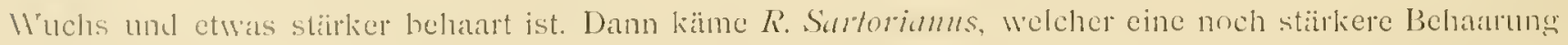
und schmälere Blattzipfel aufweist und nach Alurbeck die Velez planina in der Heregovina, ferner den ScarJus nehut cinigen Bergen Griechenlands bewohnt, und weleher auf seinem östlichsten Standolte am thessalischen (Hymp, dureh die schmiilsten Blattzipfet sich auszeichnet. Lom Oeta kenne ich endlich eine Zavisehenform mit den schmalen Phattzipfen des $R$. Sartorianns und die geringe Behatrung des R. concinuwlus. Diesen schliesst sich zuletzt $R$. demissus var. gracus Poiss. an, der aber nach boissier nicht- 


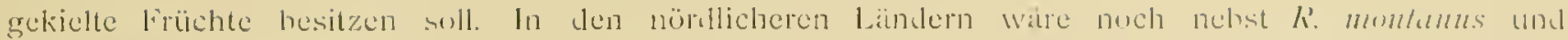

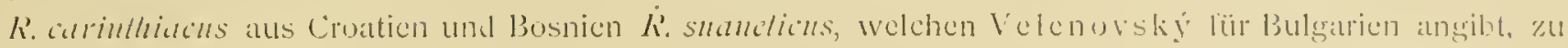
cruablhnen.

R. concimmalus kenne ich aus: Montenegro rom Berge Loveen, leg. Maly (Wiener Hofmuseum) und rom lierge Gradiste in Districte kolasin, leg. 13aldacei lil. cxs. Cornig. a. 1891, Nr. 17:3; dann rum lierge

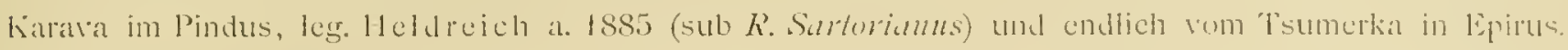
lis ist mit Sicherheit anzunchmen, dass or auch auf den albanesisehen (iebirgen nuch itulgelunden werien wirl.

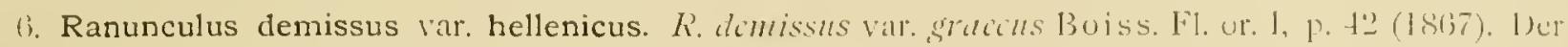
Name musste geäblert werden, da es sehon cinen K. graious Griseb. Spic. Fl. Rum. und Bithyn. 1,

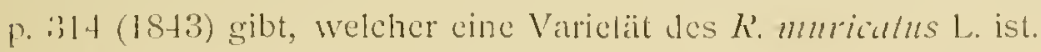

An Schnecfeldern des Gipfels vom Peristeri. Iöhe 2290 m.

lïin kJeines $3-5 \mathrm{~cm}$ hohes Pflänzchen, welches ich sowohl hicr, als auch for lünf Jahren aul de! Kional stets am Rande der. Schneelekler und beidemale nur im Stadium der Anthese vorfand, hei welelane ich daher auch ausser Stande bin anzugehen, ob die frülohte an hande gelielt oder, wic es buissier vern scinem K. demissus var. gracus angibt, oder abgestumptt sind. Ich müchte auch deshalb nur mit cince gewissen Reserve dasselbe dem R. domissus var. gracus zuzählen und die Möglichlieit, dass es als hechapine Varietät dem R. concimmalus angehüre, nicht für ausgeseblessen erachten. Jm letzteren lialle müsste diese übrigens benannt Werden, und würde ich für selbe den Namen K. concimatus var. chionophilus lorschlagen.

Nebst den beiden erwähnten Standorten, sah ich diese Varictät auch aus Mlontenegro, prope liatuni Kintica, distr. Kuci, leg. Baldacei a. IS9J und in einer etwas kräftigeren, dem hr. Sartoriums selbst sich nähernden Form als Abanien vom Berge Kandaviz im Grivas-Gebirge, leg. Baldacei, iter albanicum a. $1892, \mathrm{Ni} .10^{\prime 2}$.

7. Ranunculus neapolitanus 'len. Syll, pl. vasc. neap., p. '272 (1831). K'. Tommusimii lieichent.

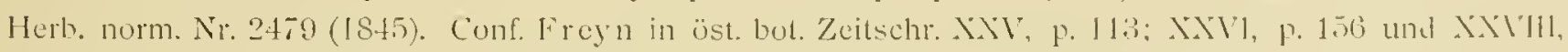
1. 72; nec nun Janka in öst. bot. Wochenbl. VI1, p. 329 und in Öst. but. Zeitschr. XXV, p. 24!).

in der Tannenregion des Tsumerka uberhab Vulgarelion. Höhe $1000 \mathrm{~m}$.

S. Nigella damascena L. Sp. pl. P. 534 (1753).

ln ker Bergregion bei Lalentini und Vulgareliun. Höhe $100-700 \mathrm{~m}$.

9. Helleborus cyclophyllus Al. Braum et Bouché Ind. sem. Berol. App. a. IS61, J. It pro var. H. viridis; Boiss. Fl. or: 1, p. 61 (1867).

Linzeln in der Bergregion bei Mazuki und Käanrytae an Fusse des Peristeri, häufiger schon in der Tammenegion des Tsumerka, massenhalt dagegen ober dieser suwohl am Tsumerka, als auch am Strungula und Peristeri, wo diese Art nebst einigen Gräisern oft weite Strecken bedeckt. Steigt bis etwat $2000 \mathrm{ml}$.

10. Delphinium halteratum Sibth. et Sm. Fl. Grace. Prodl. 1, P. 371 (1806).

Zerstreut in der Mediterranegion bei Jrta und Kalentini. Höhe $50-100 \mathrm{~m}$.

11. Delphinium Ajacis 1. Sp. pl., p. 531 (1758).

In aer Bergregrion bei Vulgarclion. Höhe $700 \mathrm{~m}$.

\section{PAPAVERACEAE U(',}

12. Papaver Rhoeas L. Sp. pl. P. 507 (1753).

In de" Bergregion bei Kalentini $(100 \mathrm{~m})$ und Kularytac $(1200 \mathrm{~m})$. 


\section{FUMARIACEAE DC.}

13. Corydalis blanda Schott, in öst. botan. Wochenbl. V1l, P. 149 (1857). Var. purpurea.

An grasigen Stellen in der oberen Region des Tsumeska oberhalb Theodoriana. Höhe $1600-180(1)$.

Ich stehe nicht an die Pflanze rom Tsumerka, nach Vergleich mit im Herbare des Wiener-Hofmuseums hefindlichen Originalexemplaren für die Schott'sche C. blanda zu halten. Es sind dies zwar nur ausschliesslich im Blüthenstadium betindliche Exemplare, aus denen es nicht ersichtlich ist, ob die Früchte, deren lorm mir bei der epirotischen Pflanze charakteristisch zu sein scheint, mit jener der letzteren ühereinstimmen. Auch gibt Schott in seiner kurzen Diagnose a. a. O., aus welcher übrigens nicht recht ersichtlich ist, auf welche Merkmale er ein besonderes Gewicht gelegt haben will, die Blüthenfarhe der Montenegriner Ptlanze für schneeweiss an, während diese hei der epirotischen, mit Ausnahme der beilcn tiefpurpurnen inneren Kronblätter, eine hell-lila ist. In der Tracht und in len übrigen Nerkmalen stimmen jedoch heide Planzen gut überein.

Von (; cal'b (l.) unterscheidet sich die von mir gesammelte Pflanze durch schmälere Blattzipfel, tiefer ausgerandete äussere und bespitzte innere Kronblätter, cinen schmächtigeren Sporn, relativ längrere Fruchtsticle (so lang oder fast so lang als die Schote) und durch kurze ovale 10-15 mm lange Schoten. Bei $C$. cal'a sind die Fruchtstiele $1 / 4-1 / 3$ so lang als die längliche $20-25$ mm lange Schote.

Zur vollständigen Klarlegung ist die Pflanze gleichwohl noch weiter zu prüfen, zu welchem Zwecke auch Samen im hiesigen botanischen Garten zur Aussat gelangen werten.

\section{CRUCIFERAE Juss.}

14. Barbarea sicula Presl. Del. Pras, p. 17 (1822).

Grasige Stellen in der oberen Region des Tsumerka oberhalb 'Theodoriana. Höhe $1800 \mathrm{~m}$.

Wurde nur im Blüthenstadium angetroffen. Ist mit sicilianischen Exemplaren von den MadonieGebirgen übereinstimmencl. Neu für die Flora der Balkanhalbinsel.

15. Barbarea bracteosa Guss. Flor. Sic. Prodr. II, p. 257 (1828).

Alpentriften des Peristeri, selten. Höhe $2000 \mathrm{~m}$.

16. Arabis caucasica Willd. Enum. plant hort. Berol. Suppl. p. 45 (1S09). - A. albidu Stev. Cat. hort. Gor., p. 51 (1812). Cf. Wettst. Beitr. Flor. Alban., p. 16-19.

Kalkielsen in der obersten Region des Peristeri. Höhe $1800-2200 \mathrm{~m}$.

17. Arabis muralis Bert. PI. rar. dec. 11, p. 37 (1806).

Kallkfelsen der Tannenregion des Peristeri bei Kalarrytae. Ilöhe $1000 \mathrm{~m}$.

18. Cardamine barbaraeoides sp. nov. Perennis glabra; rhizomate acerrimn repente, dense librilloso; caule crecto, crassiusculo, 50-60 cm alto, simplici vel apice parce ramoso; folis caulinis alternis, aeyualibus, petiolatis, unijugis, segmentis lateralibus sessifibus ovatis, segmento teminali multo majore, petiululato, wato vel subrotundo. 35-40 $\mathrm{mm}$ longo, omnibus repando-angulatis; racemis fructiferis elongatis, siliquis erecto-patentihus, pedicello duplo lengioribus, 35 m longis, in stylum brevem attenuatis. valvis planis encrviis.

An einem (iiessbache der oberen Region des Peristeri oberhalb des Dorfes Syralit. IJöhe $1500 \mathrm{mt}$

Die beschriebene Art fand jeh nur im Fruchtstadium. Sie billet am grenannten Standorte cinen äusserst dichten Bestand und besass wohl zum Theil aus letzterem Grunde die unteren Blaitter nicht mehr. Trotz dieser Umstände hin ich gezwungen, selbst aul diese uncompleten Exemplare hin sie als cine neue Art aufzustellen, dia sie cine sehr charakteristische Pllanze ist und in ihrer Tracht wesentlich von den hekunnten Caramine-Arten abweicht. Sie erinnert dureh dieselbe an Barbaraca, für welehe ich sic auch anfänglich beim Einsammeln hielt. Der nicht einnervigen Klappen wegen kann sie aber unmöglich in diese Gattung eingereiht werden und muss viehmehr zur Carduminc gezogen werden. Vermöge ihrer ansehnlichen Grösse und ihrer Blattgestalt kamn sie in dieser Gattung nur mit C. acris Griseb. verglichen werden, ron weleher 
sie sich ganz ausgezeichnet durch den langen kriechenden Wurzelstock, den gleichmatsig his zur Fruchttraube beblïtterten Stengel und durch die sämmtlich dreischnittigen B|ïtter unterscheidet. C. aris hat einen kurzen absteigenden Wurzelstock, und der Stengel dieser Art ist mit an Grösse deutlich abnehmenden entiernten, 2-5 patrig licderschnittigen Blättern besetzt.

19. Malcolmia graeca Boiss. et Sprun. Diagn. Pl. or. Ser. 1, Nr. 1, p.71 (1842). \%ُ integrifolia Boiss. Fil. ur. J, p, 228 (1S67).

In der Bergregion hei Killentini, $300 \mathrm{~m}$; dann in der Tannenregion des 'Tsumerkab oberhalh Vulgarelion unal in jener des Strungula oberhalb Melisurgi. Höhe 1000-1400 m.

20. Sisymbrium officinale L. sp. pl. p. 660 sub Erysimo (1753); Scop. 11. Carn. cd 2, 11, p. 26 (1772). In der Nähe der Dörfer Theodoriana, Melisurgi und kalarrytite. Höhe $300-1100 \mathrm{~m}$.

21. Erysimum Boryanum Boiss. et Sprun. Diagn. Pl. or. Ser. 1, Nr. 1, p. 71 (1842). Var. major Heldr. Pl. exs. Fl. Hellen. a. 1881 (e monte Pentelico).

Kallifelsen bei kalarrytae, $1000 \mathrm{~m}$.

22. Stenophragma Thalianum L. Sp. pl. p. 665 sub Arabide (175:3); Celak Prodr. Fl. Böhm. p. 44.) $(1867-1875)$.

In der oberen Region des Tsumerka oberhalb Theodoriana und des Peristeri oberhalb Chaliki. Höhe $1800 \mathrm{~m}$.

23. Aubrietia gracilis Sprun. in Boiss. Diagn. Pl. or. Ser. I, Nr, 1, p. 74 (1842).

kalk lelsen der Gipfetregion des Peristeri und des Tsumerka. Höhe 2000-2336 $\mathrm{m}$.

24. Berteroa obliqua Sibth. et Sm. Fl. Graec. Prodr. II, p. 12 sub Alysso (1813).

In der oberen Region des Peristeri unterhalb des Gipfels. Höhe $2200 \mathrm{~m}$.

25. Draba scardica Griseb. Spic. Fl. Rum. und Bithyn. 1, p. 266, pro var. D. aizoidis (1843).

Auf dem Gipfel des Tsumerka $(2336 \mathrm{~m})$ und des Peristeri $(2290 \mathrm{~m})$.

26. Draba muralis L. Sp. pl. p. 642 (1753).

In der Tannenregion des Tsumerka oberhalb Vulgarelion. Höhe $1200 \mathrm{~m}$.

27. Koniga rupestris Ten. Fl. Nap. Prodr. 1, p. XXXVIl, sub Alysso (1811-1815).

In der Gipfelregion des Tsumerka und Peristeri. Höhe 2000 m. Selten.

Galt bisher für eine endemische Art der Abruzzen. Sie scheint übrigens auf den südalbanischepirotischen Hochgebirgen weiter verbreitet zu sein, da Baldace: sic cbenfalls sammelte und zwar aul Jem Berge Stozo (Acroceraunia). Ich fand diese Art in einem Exemplare auch aul der kyllene im Peloponnes. K. scardica Griseb. ist nur durch kahle Schötchen von ihr verschicuen.

28. Alyssum montanum L. Sp. pl. p. 650 (1753).

In der oberen Region des T'sumerka oberhalb Theodoriana. Höhe $1800 \mathrm{~m}$.

29. Iberis sempervirens L. Sp. pl. p. 648 (1753).

In der oberen Region des Strungula obchalb Melisurgi $(1800 m$ ) und auf dem Giptel des Peristeri $(2250 \mathrm{~m})$.

30. Thlaspi microphyllum Boiss. et Orph. Diagn. Pl. or. Ser. II, Nr, 6, p. 19 (1859).

An Schnefeldern der oberen Region des Tsumerka und Peristeri. Höhe $2000 \mathrm{~m}$.

31. Aethionema gracile DC. Syst. II, P. 559 (1821).

In der Tannenregion des Tsumerka oberhalb Vulgarclion. Höhe $1500 \mathrm{~m}$.

32. Capsella grandiflora Bory et Chaub. Flor. Pelop. Nr. 1017, sub Thlaspide (1838); Boiss. Diagn. 1'. or. Ser. I, Nir. 1, p. 76 (1842).

Häufig in der Tannen- und Hochgebirgstegion des Tsumerka, Strungula und Peristeri, seltener in der Bergregion, wic unterhatb Melisurgi und bei Mazuki.

C. bursa pusloris $\mathrm{L}$. habe ich nirgends beobachtet. 


\section{CISTINEAE DC:}

3:i. Cistus incanus 1. Sip. pl. p. $524(175 \%)$.

Somohl in den Macehien bei Arta, als aueh in der Bergregion bei kälentini und selbst in der Tannenregron des Tsumerlia hei Vulgarelion häulig. Steigt bis $800 \mathrm{~m}$.

34. Helianthemum arcuatum Presl filur. Sic. 1, p. 128 (1826).

ln der 'lannenregion des Tsumerka hei V'ulgarelion. Höhe $1000 \mathrm{~m}$.

Die Exemplare stimmen mit der Beschreihung Presl's, mit Ausnahme in der Behaarung des Stengels. welche nicht abstehend sondern anliegend ist, gut überein. Sic sind auch mit den ron "1"odaro in f\%lure sieula exs. Nr. 741 ausgegebenen Exemplaren übereinstimmend, obzwal diese eine striegelhatrige Blattwberseite aufweisen, während jene rom Tsumerka, entsprechend der Beschreibung Presl's, eine kahle Blattuberseite besitzen. Auf diese, uffenbar durch Standurtsverhältnisse bedingten L'nterschicile in der Behaarung ist wohl kein besonderes Gewicht zu legen.

Von H. unlgare Gäitn., dem $H$. arcuatmm, wenn man will, als gengraphische Race zugezählt werden kann, unterscheidet es sich durch die länglich-lanzettlichen Stongelbläter, längere Nebenbätter und cilängliche etwas zugrespitzte Kelchziplel. Das rerwandte $H$. graecmm Boiss. und $11 \mathrm{eldr}$. ist dureh die bberscits mil zahlreichen kileinen Knötchen dichtpunktirten, striegelhatrigen Blätter verschicden.

\section{VIOLARIEAE DC:}

3.5. Viola chelmea Boiss. et Helil. Diagn. Pl. ur. Ser. 11, Nr. 1, p. 54 (1853).

Kalkfelsen der uberen Region des Tsumerkił, Höhe 2000 m, höchst selten.

Mlit der P'llanze vom Chelnuos, wo ich sie heuer ebenfalls sanmelte, volliommen identiaclr.

36. Viola aetolica Buiss. et Hel,1r. Diagn. Pl. or. Ser. 11, Nr. 6, P. 24(1859).

In Wachholderbüschen der uberen Tannenregion des Tsumerka bei Vulgarelion und an Peristeri uberhalb Kalarrytae. Höhe $1500 \mathrm{~m}$.

\section{POLYGALEAE Juss.}

37. Polygala major Jacq. Fl. Austr. V, p. 6, t. 413 (1778).

Häuthg in der Tannenregion des Tsumerka hei Vulgarelion. Höhe forom.

38. Polygala vulgaris L. Sp.pl. P. 702 (1753). Var. pindicola Hausslin. Symh. ad. Fl. Crace. P. $41 ;(189: 3)$.

ln der 'lannentegion des 'Tsumerka wherhalb Theoduriana. Ilöhe $1200 \mathrm{~m}$.

\section{SILENEAE DC}

33). Agrostemma coronaria 1. Sp. pl. 1.. 130 (1753.)

In der Bergregion bei kialentini. Höhe $100 \mathrm{~m}$.

fo. Melandrium album Nill. Gard. dict. ed. V111, Nr. 4 sub Lyehnide (17tis); Gateke lil. Nord- und Mitteldeutschl. 4. Jull., p. 5.5 (1858).

ln ker Bergregion bei Vulgarelion, Pramanta uns Mazuli. Hohe 300 -

41. Silene coesia Sibth. ct Sm. lil. Grace. l'rolr. 1, p. 294 (180).

Im Cerölle der Tannenregrion des Tsumerta oberhalb Vulgrieliun, des Strungulat bei Melisurgi. des Peristeri hei Mazuki und Kalarrytac. Höhe $1000-1500 \mathrm{~m}$.

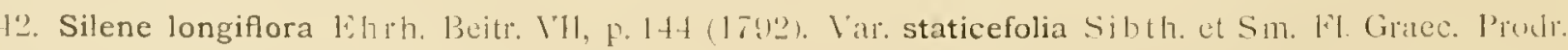

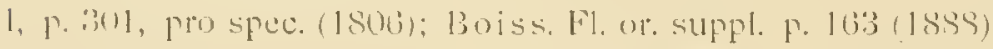

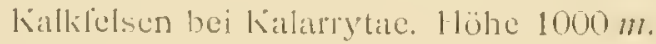


13. Silene radicosa Boiss. et 11 eldr. Diagn. Pl. or. Ser. I, Nr. 13, p. 2.4 (18.5).

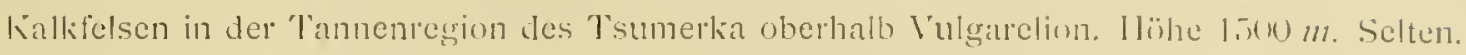

44. Silene fruticulosa Sich, in DC. Prodr. I, p. 376 (1824).

lialkfelsen der wheren Region des Tsumerka oherhalb Vulgarelion. Höhe $160 \mathrm{~m}(\mathrm{~m}$.

1.5. Silene trinervia Seb. et Maur. Fl. Rom. Prodr. p. 152 (1818).

In der Nediteranregion hei Arta und in der Bergregion bei Kalentini. Höhe .0-100 $\mathrm{m}$.

46. Drypis spinosa L. Sp. pl. p. 413 (1753). D. spinosa subsp. Limacana .1 urh. Beitr. zur Kennt Fl. Südlhosn. p. 161 (1891). D. Linnacana Murb. und Wettst. Beitr. Fl. Alban. p. 28 (1892).

In der Tannenregion des T'sumerka bei Vulgarelion und des Peristeri bei Mazuki. Hähe 1500 $\mathrm{m}$.

47. Saponaria calabrica Cuss. Pl. rar. J, p. 164 (1820).

In der Bergregion bei Kalentini. Höhe $100 \mathrm{~m}$.

48. Tunica iliyrica L. Mant. p. 70 sub Suponaria (1767): Boiss. F1. or. I, p. 520 (1867).

Kalkiflsen der Tannenregion bei kalarrytae. Höhe $1000 \mathrm{~m}$.

49. Tunica saxifraga L. Sp. pl. p. 413 sub Dinutho (1753); Scop. Fil. Carn. c.l. 2, I, p. 301)(17-2).

In der Nediterranregion bei Arta und in der Tannenregion bei kialarytac. Hähe $100-10 \mathrm{~m} / \mathrm{m}$

50. Dianthus corymbosus Sibth, et Sim. Fl. Graec. Prodr. 1, p. 2S.5 (1SOG).

In der Mediterran- und Bergregion bei Kalentini. Höhe $100-800 \mathrm{~m}$.

51. Dianthus viscidus Bory et Chaub. in Exp. scient. Moréc Jll, 22, p. 119 (1832).

In Jer Bergregion bei Kalentini. Höhe $700 \mathrm{ml}$.

lar. glabratus HeIdr. in it. IV, pel Thessal. a. 188.5. Tota planta glabrescens.

In der oberen Region des Tsumerka oberhalb Theodoriana. Höhe $1500 \mathrm{~m}$.

52. Dianthus inodorus L. Sp. Fl. P. 410 , pro var. D. caryophylli (1-Ti3); Rerner lil. exs. Austro-Hung., Ni. 543. - D. siluestris Wulf. in Jacq. Coll. 1, p. 23 r (1786).

In der Tamenregion des Strungula bei Melisurgi. Höhe $1000 \mathrm{~m}$.

5i). Dianthus integer Vis. in Florn XIl, 1, Ergäinzbl, p. 11 (1829); Fl. Dalm. t. XXXY1, Fig. 3.

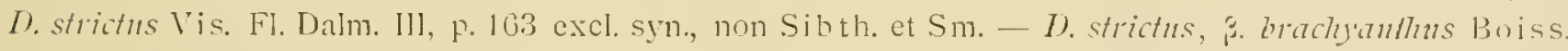
[1. ni. I, P. $486(1867)$.

In der oberen Region des Peristeri, Strungula und Tsumerka. Höhe $1800-2300 \mathrm{~m}$.

5.4. Dianthus glumaceus Bory et Chaub. in Exp. scient. Morée III, 2, p. 340 (1832). Var. obcordatus Marg. et Reut. Essai d'une Flore de l'ile de Zante, p. 31 pro spec. (1838); Boiss. Fl. or. I, p. 517.

In Jer oberen legion des Peristeri oberhalb Syraku. Höhe $1500 \mathrm{~m}$.

\section{ALSINEAE DC.}

5i). Cerastium lanigerum $\mathrm{Clem}$. Atti. del congr. Firenze, p. $520(18+1)$.

Im Crerölle auf dem Gipfel des Peristeri. Höhe $2290 \mathrm{~m}$.

7if. Cerastium Roeseri Boiss, et Heldr. Diagn. Pl. or. Ser. II, Nr. 1, p. 93 (1853). - C. brachypefalum iar. Rocseri Boiss. Fl. or. 1, p. 723 (1867).

Am Gipfel des Peristeri, Höhe $2290 \mathrm{~m}$.

Ich halte $C$. Roeseri für eine selbstïndige Art, welehe rom C. brachypclalmm Desp., mit dem selhe dic schmalrandhäutigen bis über dic Spitze hinaus behaarten lelchzipfel gemein hat, ganz und gar verschicden ist. Die Tracht und der Drüsenreichthum allein schon unterscheiden C. Rocsert leicht ron C. hrachlpchlum. Letztere Eigenschaft hat es mit C. fauricum Spreng. gemein, von welchem es (wie auch von C. machypctalum) dureh den kï̈zeren meist rom Grunde aus veristelten, dichter mit Drüsenharen besetzten Stengel, dickere Plüthensticle, grïssere Blüthen. tiefer ggetheilte (bis über die Hälfte zweispaltige) kronblätter und ungewimperte Staubfiden abweicht. 
C, luridum Ciuss., dem C. Rocseri sowohl in der Tracht, als auch in den meisten ïbrigen Mlerkmalen sehr nahe steht, ist nur durch die rerhältnissmäisig grossen, dem Kelche gleichlangen lironhlätter ron diesem verschicalen.

57. Moenchia mantica L. Sp. pl. ect. 2, p. 629 sub Cerastio (1862); Bartl. cat. sem. Cö̈t. ex lioch Syn. Fl. gem. ed. 2, p. 131.

In der Tamnenregion des Tsumerka oberhab Vulgarelion. Höhe $1000 \mathrm{~m}$.

58. Arenaria serpyllifolia L. Sp. pl. p. 123 (1753). Var. viscida Lois. Not. p. 6S pro spec. (181U). In der Tannenregion des Tsumerka und auf dem Gipfel des Peristeri. Höhe 1000 -2290 m.

50. Alsine stellata Clarlic Travels in various countr. of Europe, Asia and Afr. I1, 3, p. 211 sub Cherleria (1S16). - A. parmassica Boiss. et Sprun. Diagn. PI. or. Ser. 1, Nr. 1, p. 46 (18+2).

Var. epirota. Pedunculis glaberimis.

Auf Kallifelsen der Gipfelregion des Peristeri, selten. Höhe $2200 \mathrm{~m}$.

Von der Glundform, welche drüsige Blüthenstiele und häufig auch drüsige kelchzipfel hat. durch dic völlige Kahlheit verschieden. Unlängst erhielt ich sie auch von Herrn Oberstieutenant H. Hartl rom Berge Tringia bei Kastania im Pindus.

Clarke beschrieb a. a. O. ausführlich diese Art als Cherleria stcllala und giht als Fundort den Parnass an. Sie hat daher auch diesen Namen, als den ältesten zu führen.

60. Alsine falcata Griseb. Spic. Fl. Rum. und Bithyn. I, p. 200 (1843). - A. recmra \% mizalis Boiss. Fi. or. 1, p. 674 (1S67).

Im Gerölle der oberen Region des Strungula oberhalb Melisurgi, mit A. Gorardi (Milli.) vergesellschaftet. Höhe $1600 \mathrm{~m}$

61. Alsine Gerardi Willd. Sp. pl. II, p. 729 sub Arenaria (1799); W'ahlenb. Fl. Carp., p. 1:32 (1814).

Im Felsenschutte des Tsumerka, Strungula und Peristeri von der oberen Tannenregion bis auf die höchsten Erhebungen häufig. Höhe $1500-2336$ m.

\section{LINEAE DC.}

62. Linum liburnicum Scop. Fl. Carn. el. 2, 1. p. 230 (1772). - L corlmbulosmm Reichenh. Fl. germ. exc., p. 834 ( 1832$)$.

In der Bergregrion bei Kalentini. Höhe $100 \mathrm{~m}$.

XI. MALVACEAE R. Br.

63. Malva silvestris L. Sp. pl. p. $689(1753)$.

Verbreitet in der Mediterran- und Bergregion bei Arta, Kalentini, Melisurgi und Mazuki. Hähe $50-900 \mathrm{ml}$.

64. Lavatera thuringiaca L. Sp. pl. p. 691 (1753).

An Bïchen der Bergregrion bei Kalentini. Höhe 5oom. Selten.

(3i). Althaea hirsuta L. Sp. pl. p. 687 (17.5.3).

In der Bergregion unterhalb lialarrytac. Höhe $600 \mathrm{~m}$.

\section{HYPERICINEAE D) $\therefore$}

(i6. Hypericum Spruneri Boiss. Diagn. Pl. or. Ser. I, Nr. S, p. 11'2 (1S40).

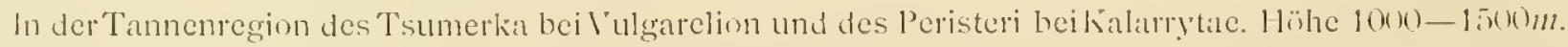

67. Hypericum barbatum Jacq. Fl. Austr. I11, p.,33 (1775). V'ar. trichanthum Buiss. et Sprun. 1)iagn.

Pl. or. Ser 11, Nr. 1, p. 10.7 pro spec. (18.3\%); Boiss. Fl. or. 1, p. 816 (1S67).

ln der oheren Region des Peristeri, sehr selten. Höhe 'zonom. 
(j8. Hypericum perforatum 1. Sp. pl. p. 78.5 (17.3.3).

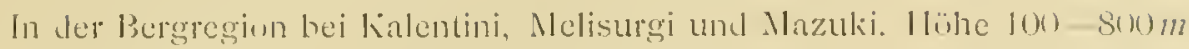

\section{ACERINEAE Dr}

(ii). Acer pseudoplatanus I. Sip. pl. p. 10.74 (17583).

In der Bergregion bei Mazuki und in der Tannentegion des Strungula wherhalb Melisurgi, cinzeln. llikle $11 x+-9(x) \mathrm{ml}$.

\section{AMPELIDEAE kunth.}

(1). Vitis silvestris (mej. Fi. Bad. 1, P. 543 (1806).

In Jur Bergregion bei Kilentini, mit krältigen Stämmen sich nuf die Bäume schlingend. Hähe 100 m

\section{XY. GERANIACEAE DC.}

7). Geranium macrorrhizum L. Sp. pl. p. 6S() (1753).

in jer oberen Tannenregion des Tsumerka oberhab liugarelion. Höhe $1600 \mathrm{~m}$.

72. Geranium subcaulescens L'Her. in DC. Prodr. I, p. 640 (1824).

Im Felsenschutte der oberen Region des'l'sumerka, Strungula und Peristeri gemein. Hoine 2000 - $230 \pi m$

73. Geranium asphodeloides Burm. Specim. bot. de Gejaniis, p. 로 (17.79).

In der oberen kegion des Peristeri oberhalb Syraku, selten. Höhe $1500 m$.

74. Geranium pyrenaicum L. Mant. p. 97 (1767).

In Jer Tannenregion des Tsumerka oberhalh Vulgarelion. Höhe $1300 \mathrm{~m}$.

75. Geranium columbinum L. Sp. pl. P. 682 (17.53).

In der Bergregion bei Pramanta. Höhe $300 \mathrm{~m}$.

76. Geranium molle L. Sp. pl. p. 68: (1753).

In der Tamnenregion bej halarytae. Höhe $900 m$.

\section{ILICINEAE Brogn.}

77. Ilex aquifolium L. Sp. Pl. P. I $2 . \overline{7}(1753)$.

Einzejn in der unteren Tannenregion bei Melisurgi. Höhe $800 \mathrm{~m}$.

\section{RHAMNEAE R. BI.}

78. Paliurus australis Gaertn. De fruct I, P. 203 (I788). - P. aculeatus Lam. Finc. meth. IS: p. $69 \%(1747)$.

In der Meliteranregion bei Arta und Kalentini. Höhe 50-100 $\mathrm{m}$.

\section{XYIII. TEREBINTHACEAE Juss.}

79. Pistacia Lentiscus L. Sp. pl. P. 1026 (I7.5:3).

In der Mediterranregion hei Arta und Kalentini. Höhe $50-100 \mathrm{~m}$.

80. Rhus cotinus L. Sp. pl. p. $20(97$ (1753).

In der Mediteranregion bei Kalentini. Höle $100 \mathrm{~m}$.

\section{PAPILIONACEAE L.}

81. Calycotome villosa V'ahl. Symb. bot. 11, p. 80 sub Spartio (179I); Link in Schrad. neu. Journ. 11, 2, p. 51 (1808).

In Macchicn bei Artil. Höhe $50 \mathrm{~m}$. 
32. Ononis spinosa L. Sp. pl. F. T1(i $(17,1,3)$.

In der Tannenregion des 'T'sumerka bei Vulgarelion und in jener bei Mazuli. Hïhe $1000 \mathrm{~m}$.

8i. Ononis antiquorum L. Sp. pl. ed. 2, p. $1006(1763)$.

In der Mediterranregion bei Arta. Höhe $50 \mathrm{~m}$.

84. Anthyllis Dillenii Schult. ap. Seringe in D) C. Prodr. 11, P. $170(18: 0.0)$.

in der Bergregion unterhaib Vulgarelion. Höhe $700 \mathrm{~m}$.

85. Medicago lupulina L. Sp. pl. p.779 (1753). Var. Cupaniana Guss. F1. Sic. sym. 11, p. 36i2 pro spec. (1843): Poiss. Fl. or. 11, p. 105 (1872).

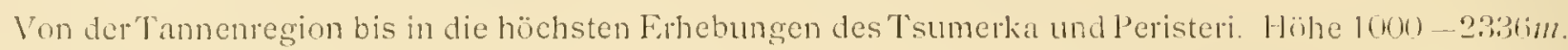

S6. Medicago falcata L. Sp.pl. p. $779(17,3,3)$.

$1 n$ der Tannenregion des Tsumerlia bei Vulgarelion. Höhe $10(x) m$.

87. Trifolium pratense L. Sp. pl. p. 768 (1753).

ln der Tannenregion des T'sumerka bei Theodoriana. Höhe $1000 \mathrm{n}$.

8. Trifolium praetutianum Guss. Pl. rar. p. 308 (1820).

Auf dem Gipfel des Tsumerlia (2336 m) und in Jer oberen Region des Strungula (1600 m).

Stimmt mit Exemplaren vom Mt. Amaro in den Abruzzen vollständig überein.

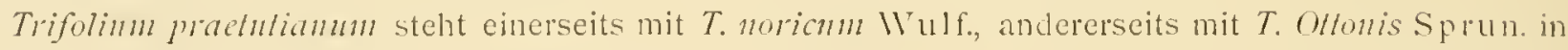
so naher Verwandtschaft, dass es schwierig hält diese drei Arten, so sehr sie auch fül den ersten Blick verschieden erscheinen, auseinander zu halten.

Die Unterschiede, mehr weniger quantitativer Art, sollen in nachstehender Tahelle ersichtlich gemacht werden.

\section{T. noricum Wulf.}

Stengel 10 - $20 \mathrm{~cm}$ hoch.

Pliitter meist lang-gestielt.

Blïttchen gross, his $3 \mathrm{~cm}$ lang. eilünglich, spärlich behaart.

Blïthen bis " $2 \mathrm{~cm}$ lang" Kelchzîhne

fast so lang als die Kelchröhre.

Kromblätter gelblichweiss.

Verbreitung: Steiermark, Kärnten, Krain, Südtirol, Venetien, Dalmatien, Montenegro, Alba. nien (Scarius).

\section{T. prachlianmm Guss.}

Stengel 5-12 cm hoch.

B]itter meist lang-gestielt.

Blättchen kleiner, bis $12 \mathrm{~mm}$ lang, eilänglich, spärlich behatart.

Blüthen bis $18 \mathrm{~mm}$ lang. Kelch. zähne fast so lang als die kelchröhre.

Kronblaitter anfangs blassgelb, Kronblätter tiefpurpun. später purpurn überlaufen.

Verbreitung: Italien (Abruzzen). Verbreitung: Nittelgriechenland Epirus.

\section{T. Ollonis Sprun.}

Stengel $1-4 \mathrm{~cm}$ hoch.

B]itter sehr liurz-gestielt.

Buattchen sehı klein, bis Smm lang, eilörmig, dicht-seidenhalrig.

Blüthen bis $12 \mathrm{~mm}$ lang. Kelchzälnne $1 / 2-1 / 3$ so lang als die lelchröhre.

(Trmpliestus, kiniax und liona).

89. Trifolium ochroleucum Huds. Fl. Angl. p. 228.3 (1762); L. Syst. ed. XIl, 3, append., p. 238 (1768). In der Bergregion bei Kalentini und in der Tannenregion bei Vulgarelion. Ilihe $8(x)-1000 \mathrm{~m}$.

90. Trifolium hirtum All. aluct. Fl. Pedem. p. 20 (1789).

In der Pergregion bei kalentini. Höhe $100 \mathrm{~m}$.

91. Trifolium purpureum Lois. F1. Gall. 11, P. 125) (1806).

An den RïnJern der Macchien bei Arta und Kilentini. Höhe 30-100 m.

(12. Trifolium arvense L. Sp. pl. p. 769 (175:3).

In der Tamnenregion des Tsumerka bei Trujgarelion. Höhe $100(1 \mathrm{~m}$.

93. Trifolium tenuifolium 'Ten. Cat. hort. Neap. p. 58 (1819).

ln der Bergregion bei kalentini und in der Tannenregion des T'sumerka bei Vulgarelion. Ilöle $13(10-10(10) \mathrm{m}$. 
9.1. Trifolium dalmaticum Vis. in lilora XII, 1, Ergänzbl. P. 2I (18201).

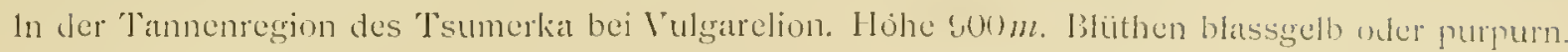

05. Trifolium striatum 1.. Sp. pl. p. $770(1753)$.

In der Tamnenregion des T'sumerka bei Vulgarelion. Hühe loun m.

96. Trifolium tenuiflorum T'en. Prodr. Fl. Neap. p. XI.IV (1S11).

In der Tannenregion des 'T'sumerka bei Vulgarelion. Höhe $1000 \mathrm{~m}$.

97. Trifolium leiocalycinum Boiss, et Sprun. Diagn. P. or. Ser. I, Nr. 2, P. :31 (1S-13). Cinf. Gibelli e Belli, Rivista crit. spec. 'Trifol. ital. in Mem. Acad. scienze li Torino, Ser. M, Tum. Alll, (Supirat.) p. 27.

Am Rande Jer Maechien bei Arta und Kảlentini. Höhe 50-100m.

9S. Trifolium Parnassi Boiss. et Sprun. Diagn. Pl. or. Ser. 1, Nr. 2, p. 30 (184:3)

In der vberen Region des Tsumerlia und Peristeri. IJ̈he $2000 \mathrm{~m}$.

99. Trifolium repens L. Sp. pl. p. 767 (1753).

In der Bergregion bei Vulgarelion und Melisurgi. Höhe $700 \mathrm{~m}$.

Vir. Orphanideum Boiss. Diagn. Pl. or. Ser. 11. Nr. 2, 17. 17 pro spec. (18506): Boiss. fil. or. 11, p. $1 \div 5$. Im Felsenschutte des Gipfels vum l'eristeri. Höhe $2200 \mathrm{~m}$.

100. Trifolium nigrescens Vis. Fl. 1tal. Fragm., p. 12 (1808).

In der Mediterranregion bei Arta und Kälentini. Höhe $50-100 \mathrm{~m}$.

101. T. campestre Schreb. in Sturm. Deutschl. Fl. Heft 10 (1804).

In der 'lannenregion des Tsumerka bei Vulgarelion. Höhe $100($ m.

1(122. Dorycnium hirsutum L. Sp. pl. p. 775 sub Loto (1753); 0 C. Prodr. I1, P. 208 (18:5).

In den Nacchien bei Arta und Kalentini, dann in der Bergregion bej Pramanta. Höhe $50-500 \mathrm{~m}$.

103. Dorycnium herbaceum Vill. Hist. pl. Dauph. 1I], p. 417 (1789). - D. intomedimm Led. Ind sem. hort. Dorpat, 1820, p. 14.

11 der 'Tannenregion des Tsumerka bei Vulgarelion und in der Bergregion bei Mazuki. Hühe 300 bis $800 \mathrm{~m}$.

Dic Kelchzipfel sind breit. drejeckig und etwa viermal kürzer als die Kelchröhre. Durch dieses Mcrk mal weichen die Exemplare von den Mitteleuropäischen ab, mit welchen sie übrigens übereinstimmen.

104. Lotus corniculatus L. Sp. pl. p. 775 (17.73).

In der Tannenregion bei Mazuki und in jener des 'Tsumerka bei Vulgăarelion. Höhe $1000 m$.

Var. alpinus boiss. Fl. or. I1, p. 160 (1872).

Im Felsenschutte aul dem Gipfel des Peristeri. Höhe '2290 m.

10.5. Lotus angustissimus 1. Sp. pl. p. $77+(1753)$.

In der Bergregion bei Kalentini. Höhe $100 \mathrm{~m}$.

106. Coronilla varia L. Sp. pl. p. 743 (1753).

ln der 'Jannenregion bei Vulgarelion und Mazuki. Höhe $1000 \mathrm{~m}$.

107. Hippocrepis glauca Ten. Fl. Neap. 11, p. $155(1811-1815)$.

In der Bergregion bei Kalentini $(700 m)$ und in der uberen Region des Peristeri ( $200(0) m$ ).

Im Blüthenstadium, in welehem dic Exemplare gesammelt sind, nur unsicher dureh die ctwas stärlicre Behatang von H. comosa L. zu unterscheiden. Visian i zieht sie auch einfach als Varictät zu dieser.

108. Colutea arborescens L. Sp. pl. P. 723 (1753).

In der Bergregion bei Kalentini und Mazuki. Höhe $100-300 \mathrm{~m}$.

100. Galega officinalis L. Sp. pl. p. T14 (175:3).

ln der Mediterranregion bei Arta. Höhe . $50 \mathrm{~m}$. 
110. Astragalus angustifolius Liam. Dict. 1, p. 321 1 (1780).

In grosser Menge dichte Pölster bildend atuf dem Gipfel des Peristeri. Höhe $2200 \mathrm{~m}$.

111. Astragalus rumelicus Bunge in Mem. Acad. Imp. St. Petersh. Ser. I'll, 'Tume NY, Ni. 1, 1'. 1:3 (1869). - A. whituchsis Boiss. Fl. or. 11, p. $319(1872)$.

In Jer Tannenregion des Strungula oberhalb thendoriana. Höhe $1500 \mathrm{~m}$.

112. Astragalus depressus L. Sp. pl. ed. 2, p. 1073 (18133).

In der oberen Region des Peristeri und 'l'sumerka. Höhe $26010 \mathrm{~m}$.

113. Onobrychis scardica Ciriseb. Spic. Fl. Rum. und Bithyn. I, p. 65. provar. O. sativae L. (1813). In der oberen Region des Tsumerka und Peristeri. Höhe $1.500-200(1) \mathrm{m}$.

Ich halte die epirotische Pflanze für O. scardica (Griseb.), la sie einerseits der Diagnose Grisebachs entspricht und da sie andrerseits mit den von Dörfler am L.jubitrn im Scaldus ggesammelten Exemplaren im 11 resentlichen übereinstinmt. Insbesondere gilt dies ron der Pflanze vom Peristeri. Die Exemplare vom 'l'sumerka haben allerdings etwas kleinere und blassere Blüthen, die Grössenverhältnisse der lilügel, des Schiffehens und der Fahne sind aber die gleichen. Ich kann sie auch aus diesem Grunte nicht für die im benachbarten thessalischen Pindus wachsende, von Haussknecht in den Mittheilungen des bot. Vereines für Gesammt-Thüringen $V$, p. 71 publicirte O. pindicola, deren Vexillum die Carina weit überragt, halten.

Simwhl die Exemplare vom Peristeri, als atuch jene vom Tsumerka weisen ührigens eine geringere Behaarung, als die rom l.jubitm.

114. Lathyrus pratensis L. Sp. pl. p. $733(1753)$.

In der Tamnenregion des Peristeri bei kialarrytac. Höhe $100 \mathrm{~m}$.

115. Orobus hirsutus L. Sp. pl. p. i2s (1753). Var. glabratus Griscb. Spic. Fl. Rum. und bithyn. I. P. $7(1)(1843)$.

In der Tannenregion des Tsumerka bei Vulgaretion. Höhe $1000 \mathrm{~m}$.

16. Vicia varia Host. Fl. Austr. 11, f. 332 (18:31).

In der Bergregion bei Kálentini. Höhe $100 \mathrm{~m}$.

\section{CAESALPINIEAE Br.}

117. Cercis siliquastrum L. Sp. pl. p. $37+1$ (1753).

In Macehien beiArta bis in die Bergregion bei kalentini. Hier oft ansuhnliche Bïume. Höhe an :30)m.

\section{AMYGDALEAE Juss.}

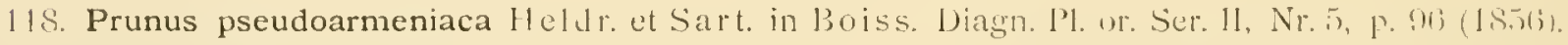

In der Tannenregion des Tsumerka bei Theodoriana und in der Burgregion hei Pramantal einzeln. llahe $300-800 \mathrm{~m}$.

\section{ROSACEAE Juss.}

11\%. Rubus ulmifolius Schott in J is 1818 , p. 821 (solum nomen).

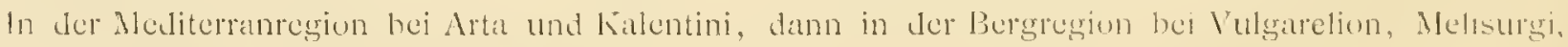
Pramantal und Mazuki. Höhe $3(0--i(0) \mathrm{m}$.

120. Rubus baldensis kiern. Nov. pl. spec. decas 111, p. 2I (1871). - R. mmifolius Sehoth $\times$ K. lomenlosus Borkh.

In der Tamnenregion oherhalb Mazuki. Höhe for) $\mathrm{m}$.

121. Rubus tomentosus Borkls. in Rocm. neu. Magaz. Bot. I, J. 2 (17) 1).

In der Bergregion bei Káalentini. Höhe $400 \mathrm{~m}$.

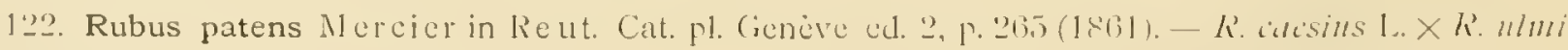
folius Schott.

In llecken bei Kalarrytale. Höhe $10(x) \mathrm{m}$. 
133. Fragaria vesca L. Sp. pl. p. I9.1 (17.33).

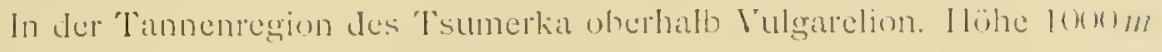

12-1. Potentilla pedata Nestl. Monogr. Potent., F. +4 (1816i).

In del oberen legegiun des l'eristeri. Höhe $1000-2100 \mathrm{~m}$.

125. Potentilla speciosa II illu. Sp. pl. 11, p. $1110(1799)$.

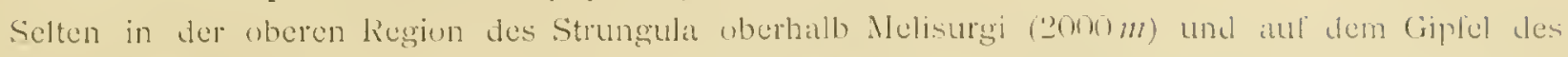

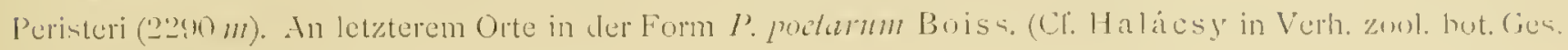
Wich XXXIII, p. 7.j).

1:6. Potentilla reptans 1. Sp. pl. p. $190,(17.33)$.

In der Tannenregion bei Melisurgi und Vulgarelion. Ilöhe $800-10 m(1) m$.

127. Potentilla micrantha Ram. in DC. Fl. Fr. IV, F. f6s (1815).

An Felsen im Dorfe Melisurgi. Hüle $800 \mathrm{~m}$.

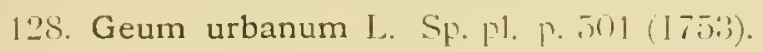

In der Bergregion bei Vulgarelion. Höhe $600 \mathrm{~m}$.

129. Rosa sempervirens 1. Sp. pl. p. t12 (1753).

In der Meiliteranregion hei Kalentini. Höhe $100 \mathrm{~m}$.

130. R. glutinosa Sibth. et Sm. Fl. Graec. Prodr. 1, p. 3-1S (1806).

In der oberen Region des Tsumerka und Peristeri. Höhe 1700 ng(x) m.

131. Rosa dorica Br. et HaI. in Verh. zoul, but. Ges. Wien, IXXVIII, p. 758 (1885).

In der Tannenregion des T'sumerka oberhalb Theodorianil. Hobe $15(1) m$.

lst mil der Pllanze der Kiona völlig übereinstimmend. Der Diagnose a. a. O. Wïre nuch beizulügen petalis mediocribus albis.

133. Agrimonia eupatoria L. Sp. pl. p. tts (1753).

In Jer Bergregion bei Kóantini und Mazuki. Höhe $200-300 m$.

133. Alchemilla vulgaris [. Sp. pl. p. $123(1753)$.

In der oberen Region des Tsumerka oberhalb Theoduriana. Höhe $180 \mathrm{~m}$.

1:34. Poterium Sanguisorba 1. Sp. pl. p. $09 \pm(1753)$.

ln der Tannenregion bei Vulgarelion. Höhe $800 \mathrm{~m}$.

\section{POMACEAE Juss.}

13). Pirus communis L. Sp. pl. p. 47! (1753).

In Aer Bergregion oberhalb Kalentini. Hohe $600 m$.

1:36. Pirus anygdaliformis Vill. Cat. method. pl. jart. Strash.. p. 32.. (1817).

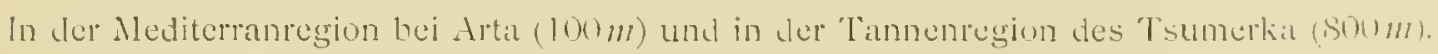

1:37. Cotoneaster parnassica Boiss. et Heldr. Diagn. I1. $11 \%$. Ser. II, Nr. 22, P. - tS (18.36).

Auf dem Giplel des Peristeri, schr selten. Höhe "2’00m.

Ist von C. fomintosa (Ait.) liamm rerschicden.

\section{MYRTACEAE R. Bi.}

138. Myrtus communis L. S.j. pl. p. 471 (1753).

In Macchien bei kialentini. Höhe $60 \mathrm{~m}$.

\section{OENOTHEREAE EndI.}

139. Epilobium parvifiorum Schrcb. Spic. Fl. Lips. P. $14(6$ (176).

An Bächen der wheren Region des Peristeri oberhalb Syraku. Höhe $1500 \mathrm{~m}$. 


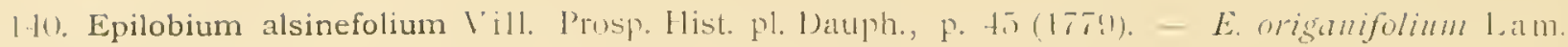
Dict. 11, p. 3360 (1-0)

An Bäiehen der oberen Region des Peristeri oherhalb Syraku. Höhe ling $\mathrm{m}$.

XXYI. PARONYCHIEAE St. Hil.

111. Herniaria incana Lam. Dict. 111, p. $12+(1789)$.

In der Tamnenregion bei Kalarytae. Höhe $800 \mathrm{~m}$.

142. Herniaria parnassica Heldr. et Sart. in Boiss. Diagn. Pl. or. Sor. 11, Nr. 1, p. (1) (18nis).

tuf dem Gipfel dus Peristeri. Höhe $2290 \mathrm{~m}$.

\section{XXYII. SCLERANTHEAE Link:}

143. Scleranthus neglectus Rochel in Baumg. Enum. stirp. Migno-lranssilv. 111, p. 34. (1816). S. marginalus Guss. Prodr. Fl. Sic., p. $486(1827)$.

In ler oberen Region des Tsumerka und Peristeri. Höhe $2000 \mathrm{~m}$.

Bildet kleine, dichte. der Erde anliegende Polster und ist durch die dichtstehenden linealen gekrümmtun Blïtter, den gedrüngten Bläthenstand und die von einem erhabenen grünen Nerven durehzogenen, breit hïutig berandeten Kelchzipfel ausgezeichnet. Dic Art ist auf den Hochgebirgen der Balkanhalhinsel jeclenfalls weit verbreitet. lch fincle sie angegeben: für Griechenland auf dem Pentelicon in Attical (leg. O)phanides), dem Delphi auf Eubnea (leg. Heldreich), auf dem Tymphrestus (leg. Heldreich), lem Killava im Pindus (leg. Heldreich). Albanien: Grivas-Gebirge (leg. Baldacei), Ljubitm im Scardus (leg. Uörfler). Macedonien: auf dem Peristeri, bei Brusnik und Kazania (leg. Formanek), auf dem Korthiati (leg. Orphanides). Auf Samothrake (leg. Degen). Bulgarien: Vitus, Rilo, Osogorska planina (leg. Velenorsky). Serbien: auf dem Kopaonik (leg. Pancicé). The nördliche Grenze erreicht sic auf den Gebirgen des Banates und Siebenbürgens. Offenbar gehört hieher auch zum Theil S. perennis Griseb. Spic. 1, p. 217.

Mir vorliegende Exemplare des S. marginatus Giuss, von Todarn in Fl. Sicula, exs. Nr. 1278, atus dem Madonie-Gebirge ausgegeben, unterseheiden sich in keiner Beziehung von der Pllanze der Balkinhalbinsel. Diese Verbreitung des $S$. Megleclus bildet neuerdings einen Belug für vine einst stattgehabte Lindrerbindung der apenninischen- mit der Balkanhalbinsel.

\section{XXYIII. CRASSULACEAE D)}

14. Sempervivum assimile Schott in üsterr. bot. Wochenll. 111, p. 1!) (1853).

Auf der Spitze des 'Tsumerka $(2336 \mathrm{~m})$ und des Peristeri $(2290 \mathrm{~m})$.

11.5. Sedum anopetalum DC. Rapp. roy. hot. 11, p. 80 (1813).

In der Tannenregion des Tsumerkab uberhalh Vulgarelion. Höhe $1000 \mathrm{~m}$

116. Sedum acre 1. Sp. pl. p. 432. (1753).

In der Tamnenregion des T'sumerka obenhalb Vulgatrelion. Höhe $1000 \mathrm{~m}$.

147. Sedum album L. Sp. pl. p. 43'? (1753).

In der Tannenregion des Tsumerka oberhalb V'ulgarclion. Höhe $1000-15010 \mathrm{~m}$.

148. Sedum dasyphyllum 1. Sp. pl. p. 4:3l (175:i).

In der Tannenregion des Tsumerkat oberhalb Vulgatrelion. Thähe $190(1) \mathrm{m}$.

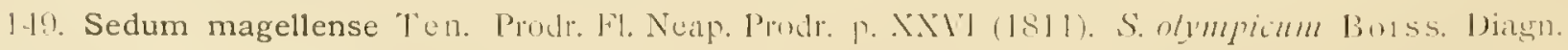

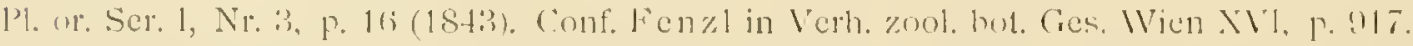

In der wberen Rugion des Peristeri wherhalb Chaliki. Ilöhe lsoom.

1.50. Sedum Cepaea L. Sp. pl. p. 4331 (1753).

In der Burgregion bei Kalentini, Vulgarelion und Mazuki. Höhe $1100-500 \mathrm{~m}$. 


\section{SAXIFRAGACEAE I) ('.}

1.)1. Saxifraga Aizoon Jilcy. F1. Austr. I, p. 18 (1778). Vil. orientalis Engl. Monogr. Saxifl:, P. $245(187:)$

In del oheren Region des 'l'sumerka, Strungula und Peristeri. I löhe 180()_.23:30m.

1.).'. Saxifraga coriophylla (iriseh. Spic. Fl. Rum. nnd Bithyn. 1, P. 3:303 (18.1:3).

Auf dem Gipfel des 'Psumerka (2033(im) und Peristeri (20)0 m).

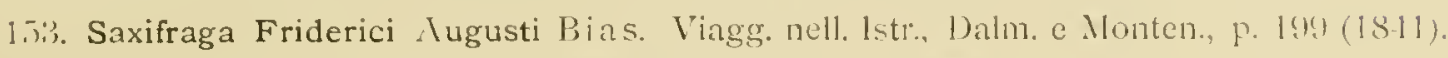

Aul dem Giplel des Tsumerlia (2336 $m$ ) und Peristeri (2200 m).

15)-1. Saxifraga tridactylites L. Sp. pl. p. 404 (17.5:3).

In der Tannenregion des T'sumerka oberhalb Vulgarelion (1500m) und in der oberen Region des Peristeri ( $2000 \mathrm{~m})$.

1,i. Saxifraga adscendens L. Sp. pl. p. 40.5 (1753). Var. parnassica Poiss. et Heldr. Diagn. P1. (1\%. ser. II, $\mathrm{Nr}$. 2, p. 69 pro spec. (1850).

In der oberen kegion des Peristeri und Tsumerka. Höhe 2000-2336 201.

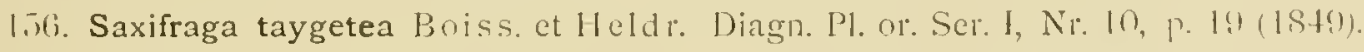

In der oberen Region des Strungula und Peristeri. Höhe $1800-2200 m$.

\section{UMBELLIFERAE Juss.}

15.). Orlaya grandiflora L. Sp. pl. P. 240 sub Caucalide (175:3); Hoffm. Gen. Unhellif., p. 5.8 (181.1) In der Tannenregion hei Theodoriana, Mazuki und Kalarytae. Höhe $800-1100 \mathrm{~m}$.

15.8. Daucus carota 1. Sp. pl. p. 242 (1753).

In der Mediteranregion bei Kalentini. Höhe $100 \mathrm{~m}$.

159. Turgenia latifolia L. Sp. pl. P. 240 sub Tordylio (1753); Hoftm. Gen Umbellif., p. 50 (1814). In Jer Tannenregion bei Kalarrytae. Höhe $1140 \mathrm{~m}$.

161). Torilis arvensis Huds. Fl. Angl., P. 98 sub Caucalide (1762). - Cancalis helucliar Jacq. Hort Vind. 111, P. 12 (17,6).

In der Bergregion oberhalb Kilentini. Höhe j00 m.

11;. Torilis nodosa L. Sp. pl. p. 240 sub Tordỵlio (17,33); Gärtn. Fruct. 1. p. 822 (178S).

In der Tannenregion bei Kalarrytae. Höhe $600 \mathrm{~m}$.

16.). Ferulago nodosa L. Sp. pl. P. 240 sub Peucedano (17.53); Boiss. Diagn. P1. o1. Ser. 1, Nr. 10. p. 37 (184i).

In der oberen Region des Peristeri oberhalb Kalarrytae. Höhe $1200 \mathrm{~m}$.

163. Peucedanum vittijugum Boiss. Fl. ol. II, P. 1018 (1972).

In der Bergregion oberhalb Kalentini. Höhe $500 \mathrm{~m}$.

164. Malabaila aurea Sibth. et Sm. Fl. Grace. Prodr. 1, P. 192 suh Heracleo (1806); Boiss. Fl. (1). 11, p. $105 \%(1872)$.

In der Tannenregion bei Kalarytae. Höhe $800 m$.

16.5. Athamanta chiliosciadia Boiss. et $11 \mathrm{eldr}$. Diagn. Ser. 11, Nr.2, p. $80\left(18.70^{2}\right)$.

Kalkfelsen der Tannenregion bei kalarytac. Höhe $1100 \mathrm{~m}$.

160. Freyera cretica Boiss. et Heldr. Diagn. P1. or. Ser. I, Nr. 10, p. 30 sub Butinia (1St9); Boiss. et Helir, 1. c. Ser. II, Nir. 2, p. 101 (18.56).

Im Felsenschutte der oheren Region Jes Peristeri. Höhe $2000 m$.

Die Exemplare stimmen somohl mit der Diagnose Boissier's und Heldreich's, als auch mit den Exemplaren, Welche Heldreich auf Jem Lassiti-Cehirge sammelte, ïherein, nur sind die Dolden 


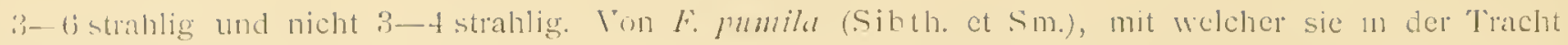
wollstïndig gleich ist, unterscheidet sic sich durch die gewimperten Blattscheiden und die glatten Mericarpien. Diese Merkmale scheinen jedoch nicht durchgreifend zu sein, da crstens einzelne Exemplare rom Peristeri ungewimperte Blattscheiden und kahle Stengel hesitzen, während andere wicder zwar gewimperte Blattscheiden, aber kuhle Stengel aufweisen und wieder andere gerimperte Blattscheiden und Stengel haben und weil zweitens bei F. pumila auch nicht alle Früichte an den Rippen von lileinen llïckchen rauh angetroffen werden. Ob sich die Sache bei der Cretenser Pllanze cobenso verhailt, vermag ich nach Jem mir vorliegenden kargen Materiale nicht zu beurtheilen; wenn Jies jeduch ler Fall wäre, so kïmnte das Artenrecht der F. crolica kaum weiters aufrechterhalten werden.

Da ich bei den Exemplaren rom Peristeri nur glatte Mericapien, häulig jedoch gewimpenc Plattscheiden angetroffen habe, so zähle ich indessen diese zu F. crelica.

167. Ptychotis ammoides Gouan. 111. und Obs. bot., p. 16 sub Seseli (17-33). Koch in nova actal Phy's. med. Acad. Leopold. Carol. Xili, I, p. 125 (1824).

Auf Hügeln in der Mediterranregion bei Arta.

168. Pimpinella Tragium Vill. Prosp. hist. pl. Dauph., p. 21 (1779).

In der Tannenregion des Tsumerka oberhalb Vulgarelion. Höhe $100(1) \mathrm{m}$.

160. Pimpinella peregrina L. Sp. pl. P. $264(1753)$.

In der Mediterranregion bei Kalentini. Höhe $100 \mathrm{~m}$.

170. Carum rupestre Boiss, et Heldr. Diagn. pl. or. Ser. 11, Nr. 2., p. T9 (1850).

Im Felscnschutte auf dem Gipfel des Peristeri. Höhe 2'290 $\mathrm{m}$.

171. Carum meoides Griscb. Spic. Fl. Rum. und Bithyn. I, p. 302 sub Silao (1843). - C. graccum Boiss. ct Fleldr. Diagn. Pl. or. Ser. 1, Nr. 6, p. 58 (1845).

Mit vorigem im Felsenschutte auf dem Gipfel des Peristeri. Höhe $2290 \mathrm{~m}$.

17.2. Trinia pumila L. Syst. veg. ed. X, p. 962 sub Seseli (17.99); Kern. Sched. wh Fl. Austro-Iung. exs. IV, p. 41 (1886).

In der obersten kegion des Peristeri und Tsumerka. Höhe 2000_2200 $\mathrm{m}$.

173. Bupleurum exaltatum M. a. Bicb. Beschreib. Länder Casp. Meere, p. 164 (1800).

In der Bergregion bei Kalarrytac. Höhe $500 \mathrm{~m}$.

174. Bupleurum semidiaphanum Boiss. Diagn. Pl. or. Ser. 11, Nr. 6, p. 73 (1859).

In der Mediterranregion bei Arta und Kalentini. Höhe 50- $100 \mathrm{~m}$.

175. Eryngium multifidum Sibth. et Sm. Fl. Cirace. Prodr. I, p. 175 (1806). - E. amellys stimm F lomifolimm Boiss. Fi. or. II, p. S26 (1872).

In der Tannenregion bei Vulgarelion und in der oberen Region des Tsumerkat und Peristeri, truppenwcise. Höhe $800-1600 \mathrm{~m}$.

176. Eryngium campestre L. Sp. pl. p. 233 (1753).

Häufig in Jer Mediterranregion bei Arta und Kalentini, dann in der Berg- und Tannenregikn bei lulgarelion, Mazuki bis in dic obere Region des Peristeri. Hö̈he 20-1500 $\mathrm{m}$.

177. Eryngium creticum J.am. Dict. IV, p. Tist (1897).

In der Meditcrantegion hej Arta und Kalentini. Höhe $50-100 \mathrm{~m}$.

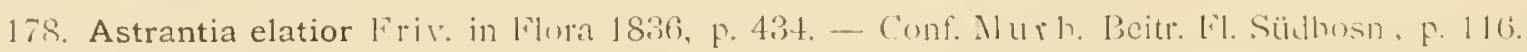

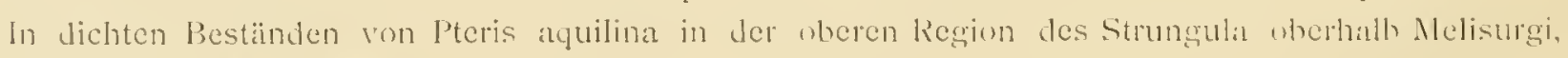
höchst selten. IÏ̈he $1.500 \mathrm{~m}$

XXXI. ARALIACEAE JuSs.

17.). Hedera Helix [. Sp. p]. p. 202 (17-5i3).

In der Bergregion bei Kalentini und Mazuki. Höhe ino $m$. 
XXXII. CORNEAE DC.

180. Cornus mas L. Sp. pl. p. 117 (1753).

In der Bergregion bei Kalentini und Mazuki strauchartig und einzeln. Höhe $500 \mathrm{~m}$. Bei Chaliki am Fusse des Peristeri baumartig, cinen ansehnlichen Bestand bildend.

\section{CAPRIFQLIACEAE Rich.}

181. Sambucus nigra L. Sp. pl. p. 269 (1753).

In der Tannenregion bei Melisurgi. Höhe $800 \mathrm{~m}$.

182. Sambucus ebulus L. Sp. pl. p. 269 (1753).

In der Tannenregion bei Melisurgi. Höhe $800 \mathrm{~m}$.

183. Lonicera etrusca Santi Viagg. I, p. $113(1795)$.

In der Mediterranregion bei Kalentini. Höhe $100 \mathrm{~m}$.

\section{RUBIACEAE Juss.}

184. Putoria calabrica L. fil. Suppl. pl. syst. veg. p. 120 sub Asperula (1781); Pers. Syn. pl. 1, p. 524 $(180 \overline{5})$.

Auf Conglomeratfelsen in der Bergregion bei Kalentini und Melisurgi. Höhe $500 \mathrm{~m}$.

185. Galium Mollugo L. Sp. pl. p. 107 (1753). Var. angustifolium L eers. Herbor. p. 115 (1775). Conf. H. Braun in Öst. bot. Zeitschr. XLII, p. 161.

In der Tannenregion des Tsumerka bei Vulgarelion. Höhe $900 \mathrm{~m}$.

186. Galium laconicum Boiss. et Heldr. Diagn. Pl. or. Ser. I, Nr. 6, p. 66 (1845).

In Jer Bergregion bei Kalentini. Höhe $500 \mathrm{~m}$.

187. Galium plebeium Boiss. et Heldr. Diagn. Pl. or. Ser. 1I, Nr. 2, p. 116 (1856).

Im Felsenschutte des Gipfels vom Peristeri. Höhe $2290 \mathrm{~m}$.

188. Galium verum L. Sp. pl. p. 107 (1753).

In der Bergregion bei Kalentini und Vulgarelion. Höhe $500-700 \mathrm{~m}$.

189. Galium anglicum Huds. Fl. Angl. ed. 2, I, p. 69 (1798).

In der Bergregion unterhalb Kalarrytae. Höhe $400 \mathrm{~m}$.

190. Galium cruciata L. Sp. pl. p. 1052 sub Valantia (1753); Scop. Fl. Carn. ed. 2, I, p. 100 (1772). In der Tannenregion des Tsumerka oberhalb Vulgarelion. Höhe $1200 \mathrm{~m}$.

191. Galium pedemontanum All. Auctuar. ad Fl. Pedem. p. 2 (1789). Var. retrorsum DC. Prodr. IV, p. $60 \bar{s}$ pro spec. (1830). Conf. Kerner in Öst. bot. Zeitschr. XX, p. 331 und Rouy Suites à la Fl. de France I. p. 112 .

In der Tamnenregion des Tsumerka oberhalb Vulgarelion. Höhe $1200 \mathrm{~m}$.

192. Valantia aprica Sibth. et Sm. Fl. Graec. Prodr. I, p. 90 sub Galio (1806). Conf. Bald. in Malpighia VlI, (1893).

In der oberen Region des Tsumerka und Peristeri. Höhe 2000-2200 $\mathrm{m}$.

103. Asperula chlorantha Boiss. et Heldr. Diagn. Pl. or. Ser. II, Nr. 6, p. 90 (1859).

An Felsen der Tannenregion und der angrenzenden oberen Region des Peristeri bei Kalarrytae. Höhe 1000-1200 $\mathrm{m}$.

194. Asperula longiflora IV. et K. Pl. rar. Hung. II, p. 162 (1805). Var: condensata Heldr. Exs. Nr. 3299, e Mt. Veluchi a. 1857. Conf. Wettst. in Biblioth. bot. Heft 26. p. 5 ?.

In der oberen Region des Tsumerka, Strungula und Peristeri. Höhe 1500-2000 m.

195. Sherardia arvensis L. Sp. pl. p. 102 (1753).

In der Tannenregion bei Vulgarelion. Höhe $1000 \mathrm{~m}$.

(v. Haláesy.) 


\section{XXYY. VALERIANEAE DC.}

196. Centranthus Sibthorpii Heldr. et Sart. in Boiss. Diagn. Pl. or. Ser. H, Nr. 2, p. II!) (I856). Kalkfelsen in der Tannenregion bei halarrytae. Höhe $1000 \mathrm{~m}$.

197. Valerianella coronata L. Sp. pl. ed. 2, p. 48 pro var. V. Locustae (1762); DC. FI. Fr. I1, p. 241 $(180 \overline{1})$.

In der oheren liegion des Peristeri bei Kalarrytae. Höhe $1200 \mathrm{~m}$.

\section{XXYVI. DIPSACEAE Vaill.}

118. Callistemma brachiatum Sibth. et Sm. Fl. Graec. Prodr. I, p. 83 sub Scabiosa (1806); Boiss. Fl. or. 111, p. $146(1875)$.

In der Mediterranregion bei Kalentini. Höhe $100 \mathrm{~m}$.

109. Scabiosa crenata Cyr. Pl. rar. Neap. p. 11 (1788).

In der Tannenregion des Tsumerka bei Vulgarelion und Theodoriana. Höhe $800-1000 \mathrm{~m}$.

200. Scabiosa tenuis Sprun. in Boiss. Diagn. Pl. or. Ser. I, Nr. 2, p. $114(1843)$.

In der Mediterranregion bei Arta und Kalentini $(50-100 \mathrm{~m})$ und in der Bergregion bei Vuigarelion und Mazuki $(500 \mathrm{~m})$.

201. Knautia hybrida A11. Auctuar, ad F1. Pedem. p. 9 sub Scabiosa (1789); Coult. Dipsac. p. 30 (1823).

Häufig in der Mediterran- und Bergregion bei Arta, Kalentini und Mazuki, bis in die Tannenregion bei Vulgarclion und kalarrytae. Höhe $50-1000 \mathrm{~m}$.

\section{COMPOSITAE Vaill.}

202. Doronicum cordatum Wulf. in Roem. Arch. 1II, p. 408 sub Arnica (1803); Schultz. Bip. in Öst. bot. Wochenbl. IV, p. 411 (1854). - D. Colummae Ten. Fl. Nap. Plodr. p. XLIX (1811). - D. corlifulium Sternb. in Denkschr. bayer. Ges. Regensb. Il, p. 147 (1818).

In der oberen Region des Peristeri, Strungula und Tsumerka. Höhe $1900-2336 \mathrm{~m}$

203. Senecio thapsoides DC. Prodt. VII, p. 301 (1838). - Cacalia ierbascifolia Sibth. et Sm. Fl. Gracc. Prodr. 11, p. $16+$ (1813), non Senecio verbascifolins Jacq.

In der oberen Region des Tsumerka und Peristeri häufig. Höhe $1500-2000 \mathrm{~m}$.

204. Senecio rupestris $\mathrm{W}$. et K. Pl. rar. Hung. II, p. 136 (1S0.5).

In der oberen Region des Tsumerka und Peristeri. Höhe $1500-2336 \mathrm{~m}$.

20.5. Senecio vulgaris L. Sp. pl. p. 867 (1753).

Auf dem Gipfel des Tsumerka. Höhe $2336 \mathrm{~m}$.

206. Anthemis tinctoria L. Sp. pl. p. 896 (1753).

In der Mediterranregion bei Kalentini. Höhe $100 \mathrm{~m}$.

207. Anthemis brachycentros Gay in Koch Syn. p. 414 (1St3).

In der Bergregion bei Kalentini. Höhe $500 \mathrm{~m}$.

208. Anthemis arvensis L. Sp. pl. p. 894 (175:3).

In der Tannen- und wberen Region des Tsumerka, Strungula und Peristeri. Höhe 1000-1600 m.

20!). Anthemis Cotula L. Sp. pl. p. 891 (1753).

In aer Bergregion bei lialentini. Höhe $500 \mathrm{~m}$.

260. Achillea Fraasii Schultz-Bip. in Flora 1842, 1, Beibl. p. 159. A. mita Spruner in Fl. 1842, p. 13.38.

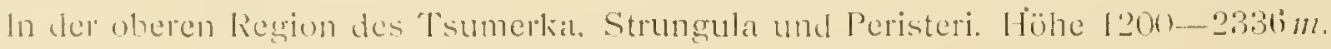


211. Achillea Kerneri. Nova hybrida. A. Frasii Schulz-Bip. $\times$ A. Clavenac L. var. integrifolia mihi. - Plarmica Kemeri mihi in Sched.

Adpresse albotomentosa, caulibus crectis simplicibus, tenuiter strinlatis, decrescenti foliatis, supcrne subaphyllis; foliis turionum et hasis caulis petiolatis, ambitu oblongis, pinnati-vel bipinnati-sectis, laciniis lincaribus integris; foliis caulinis ublongis, sessilibus, pinnatisectis, laciniis lincaribus integris; corymbo subcomposito, pedunculis capitulis ad duplo, inferioribus ad triplo longioribus; capitulis magnis, involucri pilosi phyllis ovatis acutiusculis, margine scariosis brunneis; paleis lanceolatis, margine late hyalinis, in apice sparse hirtulis; ligulis albis, late obovato lotundatis, tricrenatis vel integris, involucrum aequantibus.

Caulis $15-20 \mathrm{~cm}$ altus; folia turionum $5 \mathrm{~cm}$ longa; involucrum $5-6 \mathrm{~mm}$ longum; ligulae $5 \mathrm{~mm}$ longac, $5 \mathrm{~mm}$ latae.

Zwischen den Stammeltem in der oberen Region des Peristeri, höchst selten. Höhe $2100 \mathrm{~m}$.

Ein der streng intermediüren Charaktere wegen sehr leicht kenntlicher Bastart. Von A. Fraasii Schultz-Bip. durch die kleineren, meist weniger getheilten Blätter, den der längeren Köpfchenstiele wegen lockereren Corymbus, die grossen köpfchen und die grossen, mit letzteren gleichlangen Strahlblüthen rerschieden. Von A. Clavenae L., mit welcher Art die beschriebene Hybride in den liöpfchen übereinstimmt, durch das aus lïngeren Haaren bestehende Indument, rorzüglich jedoch durch die fein fiedertheiligen Blätter abweichend.

212. Achillea Clavenae L. Sp. pl. p. 898 (1753). Var. capitata Willd. Tract. de Achill., p. 15 pro spec (1789); Heimer] Monogr. Achill. in Denkschr. math.-naturw. Classe der kais. Akad. Wiss. Wien XLYIIl, p. 153 (1884).

In der oberen Region des 'T'sumerka und Peristeri. Höhe 1600-2150 m.

Var. integrifolia. Foliis cinerascentibus, obovato-oblongis, integerrimis, rarissime folium unum vel alterum dentibus paucis minutis suffultum; involucri phyllis glabrescentibus, atromarginatis.

Der vorigen Varietät zunächststehend und von ihr durch die Blattform abweichend.

In der oberen Region des Peristeri. Höhe $2100 \mathrm{~m}$.

213. Achillea setacea W. et K. Pl. rar. Hung. 1, p. 82 (1802).

In der Tannenregion bei Mlazuki und V'ulgarelion. Höhe 700-1000 m.

214. Achillea holosericea Sibth. et Sm. Fl. Graec. Prodr. I1, p. 194 (1813).

1 der vberen Region des Strungula und Peristeri. Höhe $1000 \mathrm{~m}$.

215. Achillea absinthoides. Spec. nova. E scclione Filipendulinac DC. Prodr. 1'1, p. 27.

Adpresse sericeo hirsuta, canescens; caulibus herbaceis crectis, angulosis, foliosis, simplicibus; foliis turionum radicalibusque Ionge petiolatis, ambitu ovatis, pinnatipartitis, segmentis infimis integris lincaribus, reliquis plerumque trifidis, laciniis linearibus integris; folis caulinis sursum diminutis, superioribus sessilibus pinnatipartitis, segmentis integris linearibus; corymbo terminali composito, denso; involucri ovati glabri phyrlis oblongis obtusis, margine scariosis albociliatis; paleis oblongis, obtusiusculis, hyalinis, pallidis, glaberrimis; ligulis luteis, lateobovatis, trilobis, involucrum subaequantibus.

Species valde aromatica, egregia, distinctissima, cum nulla alia comparanda. Caulis $30-45 \mathrm{~cm}$ altus; folia turionum cum petiolo 9-12 cm longa, petiolus laminam aequans; lamina $6 \mathrm{~cm}$ Ionga, $5 \mathrm{~cm}$ lata; involucrum $5 \mathrm{~mm}$ longum; ligulae $5 \mathrm{~mm}$ latae, $4 \mathrm{~mm}$ longae.

An Felsen und im Felsenschutte der oberen Region des Tsumerka oberhalb Vulgarelion, an einer einzigen "Spathes« genannten Stelle, hier jedoch häufig. Höhe $1500 \mathrm{~m}$.

216. Chrysanthemum segetum L. Sp. pl. p. 889 (1753).

In der Mediterranregion bei Kalentini. Höhe $100 \mathrm{~m}$.

217. Artemisia Absinthium L. Sp. p]. p. 848 (1753).

In der Tannenregion des Tsumerka oberhalb Vulgarelion. Höhe $900 \mathrm{~m}$.

218. Filago eriocephala Guss. Pl. rar. p. 344 (1826).

In der Mediterranregion bei Kalentini. Höhe $100 \mathrm{ml}$. 
219. Erigeron alpinus L. Sp. pl. p. $86+(1753)$.

Auf Jem Gipfel des Peristeri. Höhe $2250 \mathrm{~m}$.

220. Bellis perennis L. Sp. pl. p. $S 86$ (1753).

In der Tannenregion und oberen Region des Tsumerka, Strungula und Peristeri. Höhe $800-1500 \mathrm{~m}$. 221. Pulicaria odora L. Sp. pl. p. 881 sub Tnula (1753); Reichenb. Fl. Germ. exc. p. 239 (1830). In der Bergregion bei Kalentini und Mazuki. Höhe $500 \mathrm{~m}$.

222. Tussilago Farfara L. Sp. pl. p. 865 (1753).

An Bachen in der Tannenregion des Tsumerka bei Vulgarelion und des Strungula bei Melisurgi. Höhe $800 \mathrm{~m}$.

223. Carlina acanthifolia A11. Fl. Ped. I, p. 156 (1785).

In der oberen Region des Peristeri. Höhe $1500 \mathrm{~m}$. Noch nicht blühend.

224. Staehelina unifosculosa Sibth. et Sm. Fl. Graec. Prodr. II, p. 162 (1813).

An Felsen in der Tannenregion bei Melisurgi und Kalarrytae. Höhe $500 \mathrm{~m}$. In Kinospen.

225. Onopordum illyricum L. Sp. pl. p. 827 (1753).

In der Mediterranregion bei Arta und Kalentini. Höhe 50-100 m.

226. Chamaepeuce Afra Jacq. Hort. Schoenbrunn. II, p. 180 sub Carduo (1797); DC. Prodr. VT, p. 659 (1837).

In der Mediterranregion bei Arta und Kalentini, in der Berg- und Tannenregion bei Vulgarelion, Melisurgi und Mazuki, bis in die obere Region des Tsumerka, Strungula und Peristeri. Höhe 100-1200 $\mathrm{m}$.

227. Cirsium Acarna L. Sp. pl. p. S20 sub Carduo (1753); DC. Fl. Fr. IV, p. 111 (1805).

In der Bergregion bei Kalentini und Mazuli. Höhe $500 \mathrm{~m}$.

228. Cirsium candelabrum Griseb. Spic. Fl. Rum. und Bithyn. II, p. 251 (1844).

In der oberen Region des Tsumerka und Peristeri, noch nicht blühend. Höhe $1400 \mathrm{~m}$.

229. Cirsium arvense L. Sp. pl. p. $\$ 20$ sub Serratula (1753); Scop. Fl. Carn. ed. 2, II, p. 126 (1772). In der Tannenregion bei Theodoriana und Nazuki. Höhe $800 \mathrm{~m}$.

230. Galactites tomentosa Moench. Meth. p. 558 (1794).

In der Mediterrantegion bei Kalentini. Höhe $100 \mathrm{~m}$.

231. Carduus taygeteus Boiss. et Heldr. Diagn. Pl. or. Ser. II, Nr. 3, p. 42 (1856).

In der Tannen- und oberen Region des Tsumerka und Peristeri. Höhe 900-1500 $\mathrm{m}$.

Durch die schwächere Belileidung (die meisten Exemplare haben kahle oder fast kahle Blätter, und ihr Stengel ist nur oberwärts wollig-filzig) und die länger hedornten, winkelig-herabgeschlagenen (nicht geknickt-abstehenden) mittleren und oberen Hüllschuppen von zunächst verwandten C. mutums L., zu welchem Boissier nachträglich in Fl. or. I11, p. 516 denselben als Synonym stellt, zu unterscheilen.

232. Carduus armatus Boiss. et Heldr. Diagn. Pl. or. Ser. 1, Nr. 6, p. 104 (1845).

In der oberen Region des Peristeri und Tsumerlia. Höhe 1200-2100 $\mathrm{m}$.

233. Carduus pycnocephalus L. Sp. pl. ed. 2, p. 1151 (1763).

In der Mediteranregion bei Kalentini. Höhe $100 \mathrm{~m}$.

234. Tyrimnus leucographus L. Sp. pl. p. 820 sub Carduo (1753); Cass. in Dict. sc. nat. vol. 41, p. $335(1826)$.

In der Mediterranregion bei Kalentini. Höhe $100 \mathrm{~m}$.

235. Carthamus lanatus L. Sp. pl. p. 830 (1753).

In der Mediterranregion bei Arta und Kalentini. Höhe $50-100 \mathrm{~m}$.

236. Centaurea macedonica Griseb. Spic. F1. Rum. und Bithyn. 11, p. 240 pro var. C. paniculatac L. (1844), non C. macedonica Boiss. Diagn. Pl. or. Ser. J, Nr. 6, p. 130 (1845). - C. graeca, B maccdonica Boiss. Fl. or. 111, p. 6+14 (1875). - C. Griscbachil Nym. Consp. F1. Europ., p. 127 (1878-18s2).

In der Tannenregion des Peristeri bei Kalarrytae. Höhe $800 \mathrm{~m}$. 
237. Centaurea dissecta Ten. FI. Nap. I, p. LI (1811).

In der oberen Region des Strungula oberhalb Nelisurgi. Höle $1200 \mathrm{~m}$.

238. Centaurea Calcitrapa L. Sp. pl. p. 917 (1753).

In der Mediterranregion bei Arta und Kalentini, dann in der Tannenregion hei kalarrytae. Höhe $50-1000 \mathrm{~m}$.

239. Centaurea solstitialis L. Sp. pl. p. 917 (1753).

In der Nediterranregion bei Arla und Kalentini. Höhe 50-100 $\mathrm{m}$.

210. Crupina Crupinastrum Moris. Enum. sem. hort. Taur. 1842, p. 12 sub Centaurea; Vis. Flor. Dalm. 1I. p. 42 (1847). C. Morisii Bor. Fl. Centr. ed. 2. II. p. 292 (1849).

In der Tannenregion bei V'ulgarelion und Kalarrytae. Höhe $800 \mathrm{~m}$.

241. Sonchus glaucescens Jord. Observ. V, p. 75 (1847).

In der Mediterranregion bei Kalentini. Höhe $100 \mathrm{~m}$.

242. Lactuca muralis L. Sp. pl. p. 797 sub Prenanthe (175:3); Gaertn. de fruct. 1f. t. 158 (1791).

In der Bergregion bei Mazuki und Kalentini, dann in der Tannenregion des Tsumerka bei Vulgarelion. Höhe $500-900 \mathrm{~m}$.

243. Taraxacum laevigatum Willd. Sp. pl. III, p. 1546 sub Leontodonte (1800); DC. Cat. Hort. Monsp., p. 149 (1813).

In der oberen Region des Tsumerka und Peristeri. Höhe 1800 -2336 $\mathrm{m}$.

244. Taraxacum Steveni DC. Prodr, V'll, p. 149 (1838).

1 ln der oberen Region des Tsumerka. Höhe $2200 \mathrm{~m}$.

245. Hieracium oligocephalum Arv. Touvet. Hierac. Alp. Narit., p. 66 (1888).

Auf dem Gipfel des Peristeri. Höhe $2150 \mathrm{~m}$.

Die Exemplare stimmen sowohl mit der Beschreibung, als auch mit den in Baenitz, Herb. eulop. Nr. 5339 ausgegebenen Exemplaren Wolf's aus Sitten im Wallis, überein. Die Stengel sind 1-4köpfig.

246. Hieracium undulatum Boiss. Fl. or. III, p. 867 (1875).

Im Felsenschutte auf dem südwestlichen Abfalle des Gipfels vom Peristeri, häufig. Höhe $2150 \mathrm{~m}$.

Ich fand mit Ausnahme eines einzigen blühenden Individuums die Pflanze in Knospen vor. Sie ist mit der mir vorliegenden vom Korax identisch. Diese Art treibt verlängerte unterirdische Stolonen, an deren nach aufwärts gekehrten Spitze sich eine Blattrosette entwickelt. welche bestimmt ist, im nächsten Jahre den Blüthenstengel zu treiben.

247. Hieracium sabinum Seb. et Maur. Fl. Rom. Prodr. p. 270 (1818).

In der oberen Region des Tsumerka und Peristeri. Höhe 1500-2000 m.

248. Hieracium Bauhini Schult. Obs. p. 164 (1809).

In der oberen Region des Tsumerka oberhalb Theodoriana. Höhe $1200 \mathrm{~m}$.

249. Hieracium macranthum Ten. FI. Nap. IV, p. 114 pro var. H. Pilosellae (1830); Ten. 1. c. V, P. 190 (1835-1836).

In der oberen Region des Tsumerka oberhalb Theodoriana. Höhe $1500 \mathrm{~m}$.

250. Crepis neglecta L. Mant. pl. p. 107 (1767).

In ter Bergregion bei Kalentini, dann in der Tannen- und oberen Region des Tsumerka, Strungula und Peristeri gemein. Höhe 500-1200 $\mathrm{m}$.

251. Crepis setosa A. Hall. in Roem. Arch. 1. 2, p. 1 (1797).

In der Bergregion bei Kalentini und in der Tannenregion bei Vulgarelion. Höhe $600-900 \mathrm{~m}$.

252. Crepis Dioscoridis L. Sp. pl. ed. 2, p. 1133 (1763).

In der Bergregion bei Kalentini und Mazuki. Höhe $500 \mathrm{~m}$. 
253.) Picridium picroides 1. Sp. p1. p. 792 sul Scorzonera (1753): P. vulgare Desf. 1.1. Atl. 11, p. 221 $(1800)$.

In ter Mlediterranregion bei kalentini. Höhe $500 \mathrm{~m}$.

25t. Zacyntha verrucosa Gaert 1). Fruct. 11, p. 358 (1791).

In Aer Mediteranegion hei Kalentini. Höhe $100 \mathrm{~m}$.

255. Tragopogon balcanicus V'elen. in Abhanull. bölnm. Ges. Wiss, math.-naturw. Classe Ni. S, p. 28 (1886).

In der Tannenregion des Tsumerka oberhalb Vulgarelion, selten. Höhe 1200 m.

256. Tragopogon Samaritani Heldr. et Sa rt. in Boiss. Diagn. Pl. or. Ser. 11, Nr. 5, p. 116 (1S56).

In cler oberen Region des Tsumerka oberhalb Theodoriana. Höhe $1300 \mathrm{~m}$.

257. Leontodon cichoraceus Ten. Fl. Nap. Prodı., p. 46 sub Apargia (1811); Boiss. Fl. or. Ill, p. 729 (1875). Millina lcontodontoides Cass. in Dict. Sc. nat. XXYI. p. 90 (1824).

In der oberen Region des Tsumerka oberhalb Theodoriana. Höhe 1'200m.

258. Leontodon asper IV. et K. Pl. rar. Hung. 11, p. $11+$ sub Apargia (1805); Poir. enc. meth. Suppl. Il1, p. $453(1814)$.

In der Tannenregion des Tsumerka oberhalb Vulgarelion. Höhe $1000 m$.

259. Hypochaeris neapolitana DC. Prodr. V1I, p.91 (1838). H. radicata I. Sp. pl. p. 811 (1753), var. hetcrocarpa Moris. Fl. Sard. II, P. 487 (18+0). - H. dimorpha Ten. (non Brot).

In der Bergregion bei Kalentini. Höhe $500 m$.

260. Hypochaeris pinnatifida Cyr. in Ten. Syll. Fl. Neap, p. 405 (1831).

ln der Tannenregion des Tsumerka bei Vulgarelion. Höhe $900 \mathrm{~m}$.

261. Scolymus hispanicus L. Sp. pl. P. 813 (1753).

In der Mediterranregion von Arta bis Kalentini. Höhe 50-100 m.

262. Cichorium intybus L. Sp. pl. p. 813 (1753).

In der Bergregion bei Kalentini und in der Tannenregion bei lulgarelion und Melisurgi. Höhe $500-900 \mathrm{ml}$.

\section{CAMPANULACEAE Juss.}

263. Campanulla flagellaris nor. spec. (Sectio Medimm, Subsectio Triloculares Boiss. Fl. or. 1Il, p. 901$)$.

Perennis, glabrata, viridis: radice verticali, caule erecto, sacpissime flagella sterilia vel florigera edente; foliis minute crenulatis, radicalibus et inferioribus oblongo-spathulatis, in petiolum attenuatis, caulinis superioribus sessilibus, semiamplexicaulibus, oblongo-lanccolatis; lloribus terminalibus sessilibus capitatis. foliis floralibus ovatis breviter acuminatis bracteatis: calycis exappendiculati laciniis ciliolatis, tubo obconico aequilongis: corolla infundibuliformi, glabrata, violacea, calycis laciniis duplo longiore, ad tertian partem lobata.

Stengel bei normal entwickelten Individuen aufrecht, 15-35cm, bei den hïlufg rorkommenden putaten Formen nur $4-5 \mathrm{~cm}$ hoch und niederliegend. Bald ist nur ein einziger; bald jedoch neben diesem mehrere bogig-aufsteigende, stets kürzcre und weniger-blüthige vorhanden: häufig entspringen auch an der Basis des Hauptstengels beblätterte, niederliegende, Ausläufern ähnliche Sprosse. Durch letzteres Merkmal crhält die Pflanze einen eigenartigen Habitus und unterscheidet sich hiedureh wesentlich von de! ihr nächstverwandten C. glomcrata L. - Diese ist auch ïbrigens durch die langgesticlten, am Grunde herzfölmigen wer abgerundeten, nicht in den Blattstiel verschmälerten grundständigen und die gleichgestalteten ebenfalls gestielten, unteren stengelständigen Blätter, wie auch durch die längeren, schmäleren Bracteen und die grösseren Blumenkronen verschicken. -

line gewisse Innlichkeit hat auch die neuc Art, besonders in ihren putaten Formen, mit $C$. parmassica Buiss et Sprun. Diagn. P1. ol. Ser. 1, N1: T, P. 17, welche jeloch mit ihr nichts weiter zu thun hat, da 
letztgenannte Art (wie dies schon Boissier in Fl. or. IIl, p. 917 vermuthete) der an der Spitze sich öfnenden laapsel wegen in die Gattung Edrajanthus gehört. ${ }^{1}$

In der oberen Region des Peristeri. Höhe $1500-1500 \mathrm{~m}$. Auch in Thessalien aluf dem Zygus oberhalb Metzovo im Pindus, wo sie Heldreich 1885 sammelte und als C. glomerata vertheilte.

264. Campanula spathulata Sibth. et Sm. Fl. Graec. Prodr. I, p. 137 (1806).

In der oberen Region des Peristeri und Tsumerka. Höhe $1700-2200 \mathrm{~m}$.

205. Campanula ramosissima Sibth. et Sm. Fl. Graec. Prodr. 1, F. 137 (1806).

In der Bergregion bei Kalentini und Mazuki, dann in der Tannenregion des Tsumerka wherhalh Vulgarclion. Höhe $500-1000 \mathrm{~m}$.

266. Edrajanthus graminifolius L. Sp. pl. p. 166 sub Campanula (1753); D C. Prodr. VII, p. 448 (1839).

In der oberen Region des Peristeri, besonders aul dem Gipfel häufig. Sowohl die Varietät elatus als pusillus Wettst. Mlonogr. der Gatt. Hedraeanth. (Separat) p. 17, welche hier whne Grenze in einander übergehen. Dagegen fand ich die Var. australis Wettst. I. c. nicht.

267. Podanthum limonifolium Sibth. et Sm. Fl. Graec. Prodr. I, p. 144 sub Phyteumate (1800); Boiss. Fl. or. JII, p. 951 (1875).

In der Tannenregion des Peristeri unterhalb Kalarytae, selten $(800 \mathrm{~m})$. Viel häufiger in der oberen Region des genannten Berges und des Tsumerka.

In der Tannenregion sind dic Stengel gewöhnlich schlanker und höher, die Blüthen entfernter; in höheren Lagen wird die Pflanze meist niedriger und ihre Blätter werden mehr gewellt und schmäler, sie geht so allmählich in die Var. alpinum Boiss. 1. c. (P. repandnm Sibth. et Sm. Fl. Gracc. Prodr. 1, p. 143) über, welche daher nur als eine Zwergform zu betrachten ist.

\section{ERICACEAE LindI.}

268. Erica arborea L. Sp. pl. p. $353(1753)$.

In den Macchien bei Arta und Kalentini, einzeln auch noch in den Wäldern der Bergregion. Höhe $50-400 \mathrm{~m}$.

269. Arbutus Andrachne L. Sp. pl. cd. 2, p. 566 (1762).

In den Macchien bei Arta und Kalentini. Höhe $50-400 \mathrm{~m}$.

\section{OLEACEAE Lindl.}

270. Olea europea L. Sp. pl. p. 8 (1753).

In der Mediterranregion bei Arta gepflanzt und in den Macchien wild. Höhe $50-100 \mathrm{~m}$.

271. Phillyrea media L. Sp. pl. ed. 2, p. 10 (1762).

In den Macehien bei Arta und Kalentini bis in die Wälder ler Bergregion vordringend. Höhe $50-500 \mathrm{~m}$.

272. Fraxinus excelsior L. Sp. pl. p. 1057 (1753).

Einzein in Wäldern der Bergregion bei Kalentini. Höhe $500 \mathrm{~m}$.

\section{GENTIANEAE Juss.}

273. Erythraea Centaurium 1. Sp. pl. p. 229 sub Gentiana (1753); Pers. Syn. I, f. 283 (1805).

In der Mlediterran- und Bergregion bei Arta und Kalentini. Höhe $50-500 \mathrm{~m}$.

1 Wetlstein, der Nonograph der Gattung Edrajum/hus, dem ich dic auf der hiona von mir gesammelten Exemplare vorlegte, bestitigte nach Untersuchung der mophologisehen Verhältnisse und des auatomischen Baues derselben, vollinhaltlich meine diesbezügliche Anschaung. Die Art hat daher auch den Namen Edrajumthus parmassicus Boiss, et Sprun. (sub Campunula) zu führen. 


\section{X1.II. CONVOLVULACEAE Juss.}

274. Convolvulus silvatica IV. et K. PI. rar. Hung. III, p. 299 (1812).

$1 \mathrm{n}$ der Bergregion bei Kalentini. Höhe $599 \mathrm{~m}$.

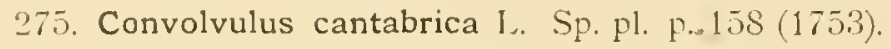

In Aer Tannen- und oberen Region des Tsumerka. Höhe $900-1100 \mathrm{~m}$.

226. Cuscuta epithymum L. Sp. pl. p. 124 pro var. C. europaeae (1753); 1. Syst. ed. N111, p. 140 (1794).

Auf Aspernla chlorantha Boiss. et HeIdr. in der Tannenregion des Peristeri bei Kalarrytae. Höhe $1100 \mathrm{~m}$.

XIIII. BORAGINEAE JUSS.

277. Echium italicum L. Sp. pl. p. 139 (175:3).

In der Bergregion bei Kalentini und Mazuki. Höhe $500 \mathrm{~m}$.

278. Echium plantagineum L. Mant. p. 202 (1771).

In der Mediterranregion bei Arta und Lalentini. Höhe 50-100 $\mathrm{m}$.

279. Onosma helveticum Boiss. Diagn. Pl. or. Ser. 1, Nr. 11, p. 111 (1849).

In der oberen Region des Strungula oberhalb Melisurgi. Höhe $1500 \mathrm{~m}$.

280. Lithospermum incrassatum Guss. Prodr. Fl. Sic. 1, p. 311 (1827).

Im Felsenschutte der oberen Reyion des Peristeri. Höhe $2000 \mathrm{~m}$.

281. Myosotis silvatica Hoffm. DeutschI. Fl. 1, p. 61 (1791).

In der Bergregion bei Mlazuki. Höhe $600 \mathrm{~m}$.

282. Myosotis alpestris Schmidt. Fl, boem. III, p. 26 (1784).

In der oberen Region des Tsumerka und Peristeri. Höhe 1800-2200 $\mathrm{m}$.

283. Myosotis arvensis L. Sp. pl. p. 131 pro var. M. scorpioidis (1753); Roth bot. Abhandl., p. 20 (1787). - M. intrmedia Link En. hort. Berol. 1, p. 164 (1S21).

In der Bergregion bei Kalentini. Höhe $600 \mathrm{~m}$.

284. Myosotis stricta Link. En. hort. Berol. 1, p. 104 (1821).

In der oberen Region des Tsumerka oberhalb Theodoriana. Höhe $1200 \mathrm{~m}$.

285. Cynoglossum Columnae Biv. Manip. 11, p. 3 (1814).

In der Bergregion bei Kalentini, dann in der Tannenregion des Tsumerka bei Vulgarelion und in der oberen Region des Strungula bei Melisurgi. Höhe 600-1500 $\mathrm{m}$.

\section{XIJV. SOLANACEAE Juss.}

286. Hyoscianus niger L. Sp. pl. p. 179 (1753).

Wüste Plätze in Kalarytae. Höhe $1100 \mathrm{~m}$.

287. Physalis Alkekengi L. Sp. pl. p. $183(1753)$.

ln der Bergregion bei Nazuki. Höhe $700 \mathrm{~m}$.

\section{XI,V. SCROFULARIACEAE Lindl.}

288. Verbascum epixanthinum Boiss. et Heldr. Diagn. Pl. or. Ser. 1, Ni. 7, p. 39 (1840).

ln der uberen Region des Tsumerka oberhalb Theodoriana. Höhe $1800 \mathrm{~m}$.

289. Verbascum Blattaria 1. Sp. pl. p. 178 (1753).

In der Bergregion bei Kalentini. Höhe $400 \mathrm{~m}$.

290. Verbascum macilentum Franchet Essai sur les esp. du genre Verbasc. P. 176 (1868). ( I. lmlrcrutulum Vill. $\times$ is: Blattariı? L.).

Unter den Eltern in der Bergregion hei Kalentini. 1 öhe $400 \mathrm{~m}$. 
291. Verbascum pulverulentum Vill. Ilist. pl. Dauph. II, p. 490 (1787).

In der Nediteranregion bei Arte, dann in Jer Bergregion bei Kalentini und in der Tannenregion des Tsumerka bei Vulgarelion. Höhe 100-1200 m.

292. Verbascum Chaixi Vill. Hist. pl. Dauph. II, p. 491 (1787).

ln der oberen Region des Peristeri bei Kalarrytae. Höhe $1100 \mathrm{~m}$.

293. Scrofularia canina L. Sp. pl. p. 621 (1753).

In der oberen Region des Tsumerka oberhalb Theodoriana und des Peristeri bei Kalarrytac. Höhe $I(x) 0-1100 \mathrm{~m}$.

294. Scrofularia laciniata W. et K. Pl. rar. Hung. 11, p. 185 (1805).

In der oberen Region des Peristeri. Höhe $2000 \mathrm{~m}$.

Var. multifida Willd. Hort. Berol. Tab. LVIIl pro specie (1816); Boiss. Fl. or. IV, p. 409) (1879).

In der oberen Region des Peristeri. Höhe $1700 \mathrm{~m}$.

Die Exemplare gehören zur Forma puberula, da sie besonders oherwärts in der Inflorescenz mit kurzen Drüsenhaaren bekleidet sind. Wettstein in Beitr. zur Fl. Alban., p. iて äussert Bedenken gegen die Identität der Willdenow'schen mit der Boissier'schen Pflanze, weil die Abbildung und Beschreibung Willdenow's nicht präcis ist und weil vom Autor kein Vaterland angegeben wird. Da jedoch Boissier zu seiner V'arietät das Herbar Willdenow's selbst citirt, er also offenbar die Exemplare auch eingesehen hat, so nehme ich keinen Anstand auf die Autorität Boissier's hin Willdenow als Autor anzuführen.

295. Digitalis lanata Ehrh. Beitr. Naturk. VII, p. 152 (1792).

In der Bergregion bei Káalentini. Höhe $500 \mathrm{~m}$.

296. Digitalis laevigata IV. et K. Pl. rar. Hung. II, p. 171 (I 805).

In der Tannenregion unterhalb Kalarrytae. Höhe $600 \mathrm{~m}$.

297. Digitalis grandiflora All. Fl. Ped. I, p. 70 (1785).

In der Tannenregion und in Pteris aquilina-Beständen der angrenzenden oberen Region des Strungula oberhalb Melisurgi. Höhe $900-1200 \mathrm{~m}$.

298. Linaria peloponnesiaca Boiss. et Heldr. Diagn. Pl. or. Ser. 11, Nr. 3, p. 163 (1856).

Im Felsenschutte des Gipfels vom Peristeri. Höhe $2290 \mathrm{~m}$.

Die Stengel sind durchwegs unverästelt und nur 10-12 $\mathrm{cm}$ hoch; die Beblätterung derselben eine seh. dichte. Die Pllanze erhält hiedurch einen eigenen Habitus und ist von in niedereren Lagen wachsenden Exemplaren, die eine riöhe bis $60 \mathrm{~cm}$ erreichen, meist verästelt und sparsamer beblättert sind, für den ersten Blick recht verschieden.

299. Linaria Pelisseriana L. Sp. pl. p. 615 sub Antirrhino (1753); Mill. Dict. ed. 8, Nr. 11 (1768).

In der Bergregion bei Kalentini. Höhe $500 \mathrm{~m}$.

300. Linaria minor L. Sp. pl. p. 617 sub Antirrhino (1753); Desf. Fl. Atl. II, p. 46 (1798).

In der Tannenregion des Tsumerka und Peristeri. Höhe $800-1000 \mathrm{~m}$.

301. Linaria graeca Bory et Chaub. in Exp. scient. Mor. III. 2, p. 175 sub Antirrhino (1832); Chav. Monogr. Antirrh., p. 108 (1833).

In der Mediterranregion bei Kalentini. Höhe $200 \mathrm{~m}$.

302. Veronica Anagallis L. Sp. pl. p. 12 (1753).

In der Tannenregion des Tsumerka bei V'ulgarelion. Höhe $800 \mathrm{~m}$.

303. Veronica Beccabunga L. Sp. pl. p. 12 (1753).

An Alpenbächen der oberen Region des Peristeri. Höhe 1500-2000 m.

30t. Veronica balcanica Velen. Fl. Bulgar. p. 431 (1891).

An Alpenbächen der oberen Region des Peristeri, mit voriger Art vermischt. Höhe $2000 \mathrm{~m}$.

(v. Malàcsy.) 
305. Veronica arvensis L. Sp. pl. p. 13 (1753).

In der oberen Region des Strungula oberhalb Melisurgi und auf dem Gipfel des Peristeri. Höhe $1500-2290 \mathrm{ml}$

306. Euphrasia puberula Jord. Pug. pl. nor. p. 133 (1852). Teste Wettstein.

In der oberen Region des Tsumerka oberhailb Theodoriana, selten. Höhe $1500 \mathrm{~m}$.

307. Pedicularis graeca Bunge in Bull. Phys. Math. Petersb. I, p. 376 (1843).

Auf dem Gipfel des Peristeri $(2290 \mathrm{~m})$ und des Tsumerka $(2330 \mathrm{~m})$.

\section{OROBANCHEAE JuSS.}

308. Orobanche nana Noe F1. di Fiume in Alman. Fiumano, p. 70 (1858).

In der wberen Region des Tsumerka oberhalb Vulgarelion, höchst selten. Höhe $1400 \mathrm{~m}$.

309. Orobanche major L. Fl. Suec. ed. 2, p. 219 (1755). O. elatior Sutt. in Trans. Linn. Soc. IV, p. $178(1797)$.

In der oberen Region des Peristeri bei Kalarrytae. Höhe $1200 \mathrm{~m}$.

\section{ACANTHACEAE Juss.}

310. Acanthus spinosus L. Sp. pl. p. 639 (1753).

In der Mediterranregion bei Kalentini. Höhe $100 \mathrm{~m}$.

\section{VERBENACEAE JusS.}

311. Verbena officinalis L. Sp. pl. p. 20 (1753).

In der Berg- und Tannenregion bei Kalentini, Vulgarelion, Melisurgi, Mazuki und Kalarrytae. Höhe $500-1000 \mathrm{~m}$.

312. Vitex agnus castus L. Sp. pl. p. 638 (1753).

In der Nediterranregion bei Arta und Kalentini. Höhe 50-100 $\mathrm{m}$.

\section{LABIATAE JuSS.}

313. Teucrium Chamaedrys 1. Sp. pl., p. 565 (1753).

In der Bergregion bei Kalentini und Mazuki. Höhe 500-600 m.

314. Teucrium montanum L. Sp. pl. p. 565 (1753). Var. hirsutum Boiss. Fl. or. IV, p. 819 (1879). T. montanmm, var. pamassicum CeJak in Bot. Centralbl. X1V, p. 153 (1883).

In der Tannenregion des Tsumerka bei Vulgarelion und in der oberen Region des Strungula oberhalb Melisurgi. Höhe $1000-1500 \mathrm{~m}$.

T. prostratum Schur. Sert. p. 60 (1853) $=$ T. pannonicum Kern. in öst. bot. Zeitschr. NI11, p. 384 (1863) ist hievon dureh einen lockereren Wuchs, etwas grössere Blüthen und breitere Blätter verschieden. Beide sind übrigens durch Zwischenformen mit einander verhunden. Der Beschreibung nach gehört auch T. Skorpilii Velen. in Sitzungsber, böhm. Ges. W'iss. 1889, p. 39 hicher.

315. Teucrium Polium L. Sp. pl. p. 560 (1753).

In der Bergregion bei Kalentini und Mazuki. Höhe $500 \mathrm{~m}$.

316. Salvia Sclarea L. Sp. pl. p. 27 (17:33).

In der Tannenregion des Tsumerka bei Theodoriana, dann in der Bergregion bei Pramanta und Mazuki. Höhe 500-1000 $\mathrm{ml}$.

317. Salvia Barrelieri Ten. Fl. Nap. I, p. 9 (1811).

In der Mediterranregion bei Kalentini. Höhe $100 \mathrm{~m}$. 
Üppige Exemplare werden his $1 m$ hoch. Eine durch die reiche Inllorescenz und die hellazurblauen Blüthen sehr aulfallende Art, weiche keineswegs mit S. pratensis L. vereinigt werden kann. Sie scheint letztere in Griechenland zu vertreten, denn ich beobachtete sie wiedcrholt in Actolien, Achaia und Arcadien, währond ich letztere nirgends angetroffen hatte.

318. Salvia amplexicaulis 1.am. I11. I, p. 68 (1791).

In der Bergregion bei P'ramanta und Nazuki, dann in der Tannenregion bei V'ulgarelion, Theodnriana und Kálarrytae. Höhe $500-1100 \mathrm{~m}$.

319. Salvia Horminum L. Sp. pl. p. 24 (1753).

In der Mediterranregion bei Arta und Kalentini. Höhe 50-100 $\mathrm{m}$.

320. Ziziphora capitata L. Sp. pl. p. 21 (1753).

Im Sande des Arachthos bei Arta, dann in der Bergregion bei Pramanta. Höhe $50-500 \mathrm{~m}$.

321. Scutellaria Columnae A11. F1. Ped. I, F. 40 (1785).

In der Bergregion bei Kalentini. Höhe $500 \mathrm{~m}$.

322. Scutellaria peregrina L. Sp. pl. p. 599 (1753). Var. adenotricha Boiss, et Heldr. Diagn. Pl. or.

Ser. 11, Nr. 4, p. 27 (1859).

In der Tannen- und oberen Region des Peristeri bei Kalarrytae. Höhe $800-1200 \mathrm{~m}$

323. Scutellaria alpina L. Sp. pl. p. 599 (1753).

Auf dem Gipfel des Peristeri. Höhe $2290 \mathrm{~m}$.

324. Prunella vulgaris L. Sp. pl. p. 600 (1753).

In der Tannenregion des Strungula bei Melisurgi und des Peristeri bei Kalarrytae. Höhe $1000 \mathrm{~m}$.

325. Prunella laciniata L. Sp. pi. p. 600 pro var. P. vulgaris (1753). - P. alla Pall. apud M. a Bieb. Fl. Taur. Cauc, 11, p. 67 (1808).

ln der Bergregion bei Kalentini und Vulgarelion. Höhe $500-700 \mathrm{~m}$.

326. Lamium pictum Boiss. et Heldr. Diagn. Pl. or. Ser. 1I, Nr. 4, p. 44 (1S59).

In der oberen Region des Tsumerka, Strungula und Peristeri. Höhe $1500-2000 \mathrm{~m}$.

327. Stachys Heldreichii Boiss. Fl. or. IV, p. 721 (1879).

In der Bergregion bei Kalentini. Höhe $500 \mathrm{~m}$.

328. Stachys penicillata Heldr. et Silrt. in Boiss. Diagn. Pl, or. Ser. I1, Nr. 4, p. 37 (1859).

In der Tannenregion des Tsumerka bei Vulgarelion. Höhe $1000 \mathrm{~m}$.

329. Stachys Reinerti Heldr. Herb. graec. norm. Nr. 743 (1858). - S. alpina $\beta$ discolor Boiss. Fl. or. 1V, p. 719 (1879). - Cf. Murbeck Beitr. zur Fl. Südbosn., F. 62 et Wettst. Beitr. Fl. Alban., p. S4.

In der oberen Region des Peristeri oberhalb Syraku. Höhe $1500 \mathrm{~m}$.

330. Stachys annua L. Sp. pi. p. 573 sub Betonica (1753); L. 1. c. cd. 2, p. 813 (1763).

In der Tannenregion des Peristeri unterhalb lialarrytae und in der oberen Region des Tsumerka oberhalb Theodoriana. Höhe $800-1200 \mathrm{~m}$.

331. Phlomis fruticosa L. Sp. pl. p. 584 (1753).

In der Mediterranregion bei Arta ausgedehnte Bestände bildend, von hier durch die Bergregion bei Lảentini bis in die Tannenregion des T'sumerka bei Vulgarelion, einzeln noch bis in einer Höhe von $1200 \mathrm{~m}$.

332. Ballota nigra 1. Sp. pl. p. 582 (1753)

In der Bergregion bei Pramanta und Mazuki. Höhe $500 \mathrm{~m}$.

333. Ballota hirsuta Willd. Sp. pl. 111, p. 113 sub Martubio (1800); Benth. Iabiat., p. 595 (1832). B. rupestris Biv. Manip. II, p. 1 sub Marubio (1814); Vis. Fl. Dalm. 11, p. $216(18+i)$.

In der Tannenregion des Peristeri unterhalb Kalarrytac. Höhe $700-800 \mathrm{~m}$. Der südlichst gelegene Standort auf der Balkanhalbinsel. 
334. Marrubium vulgare L. Sp. pl. p. 853 (1753).

In der Bergregion bei Kalentini und Mazuki. Höhe $500 \mathrm{~m}$.

335. Marrubium Frivaldszkyanum Boiss. Diagn. Pl. or. Ser. 1. Nr. 12, p. 74 (185:3).

In der Tannen- und oberen Region des Tsumerka bei Vulgarelion und Theodoriana, dann in der Tannen- und oberen Region des Peristeri bei Kảlarrytae. Hühe $800-1300 \mathrm{~m}$.

336. Sideritis Roeseri Boiss. et Heldr. Diagn. Pl. or. Ser. II, Nr. 4, p. 30 (1850).

In der Tannen- und oberen Region des Peristeri bei Kalarrytite. Höhe $900-1200 \mathrm{~m}$.

337. Sideritis purpurea Talhot in Benth. Labiat., p. $742(1832-1830)$.

In der Mediterranregion bei Kalentini. Höhe $100 \mathrm{~m}$.

338. Nepeta violacea L. Sp. pl. p. 570 (1753); Vill. PI. Dauph. II. p. 367 (1787).

In der Tannenregion des Tsumerka oberhalb V'ulgarelion. Hühe $1200 \mathrm{~m}$.

339. Nepeta Spruneri Boiss. Diagn. Pl. or. Ser. II. Nr. 4, p. 23 (1859).

In derTannenregion des Tsumerka bei Vulgarelion und des Peristeri bei Kałarrytae. Höhe $900-12(10 \mathrm{~m}$.

340. Clinopodium vulgare L. Sp. pl. p. 58 i (1753).

In der Bergregion bei Kalentini, Vulgarelion und Mazulki. Höhe $500-700 \mathrm{~m}$.

341. Melissa officinalis L. Sp. pl. p. $592(1753)$.

In der Bergregion bei Kalentini. Höhe $500 \mathrm{~m}$.

342. Calamintha suaveolens Sibth. et Sm. Fl. Graec. Prodr. 1, p. 420 sub Thymo (1806); Buiss. Fl. or. 11 , p. 582 (1879).

In der oberen Region des Peristeri bei Kalarrytae. Höhe $1100 \mathrm{~m}$.

343. Calamintha alpina L. Sp. pl. p. 591 sub Thymo (1753); Lam. FI. Fr. 1I, p. 394 (1778).

In der oberen Region des Tsumerka und Peristeri. Höhe $1800-2200 \mathrm{~m}$.

344. Micromeria juliana L. Sp. pl. p. 567 sub Satureja (1753); Benth. Labiat. p. 373 (1832-1836). In der Mediterran- und Bergregion bei Kalentini. Höhe $100-500 \mathrm{~m}$.

345. Micromeria cremnophila Boiss. et Heldr. in Boiss. Fl. or. N, p. 570 (1879).

In der Tannen- und oberen Region des Peristeri bei Kalarrytae. Höhe $1000-1200 \mathrm{~m}$.

346. Origanum hirtum Link Enum. fl. hort. Berol. Il, p. 114 (IS22). Cf. Kern. Fl. exs. Austro-hung. Nr. 181.

In der Bergregion bei Kalentini, Vulgarelion und Mazuki. Höhe $400-700 \mathrm{~m}$.

347. Thymus teucrioides Boiss. et Sprun. Diagn. Pl. or. Ser. I, Ni. 5. p. 15 (18t4). - T. gratolens Sibth. et Sm. Fl. Graec. Prodr. I, p. 421 (1806), non M. a. Bieb.

In der oberen Region des Strungula oberhalb Melisurgi einzeln (1800 m); in grosser Menge auf dem Gipfel des Peristeri. Höhe $2290 \mathrm{~m}$.

348. Thymus Chaubardi Boiss. et Heldr, Diagn. Pl. or. Ser. Il, Nr. 4, p. 6 pro var. T. angilustifolii (185!).

In der oberen Region des Tsumerka und Peristeri. Höhe $1000-1500 \mathrm{~m}$.

349. Thymus longicaulis Pres1. F1. Sic. p. 37 (1826). Cf. Kern. Fl. exs. Austro-hung. Nr. 2146.

In der Tannen- und oberen Region des Tsumerkat und in der oberen Region des Peristeri. Höhe $100(1-2100 \mathrm{~m}$.

350. Thymus Boissieri. Species nova. Syn. T. hirsutus Buiss. F1. or. 1T. p. 55 - p. p. non M. a. Bieh. (Sectio Hyphodrmae Korn. in Öst. hot. Zeitschr. XXIV, P. S5, Subsectio Isolepides Borb. Symb. ad Thy'm. Europ. p. 49).

Dense caspitosus; caudiculis procumbentibus, ramis novellis filiformibus tenubus dense foliatis, steritbus glabratis, floriferis circumcirca pilosis, axillis fasciculiferis; foliis calulnis linearibus ohtusis glabris vel brevissime hirtulis, margine revolutis pilisque longis alhis ciliatis, noralihus vix latioribus 
impunctatis; Horibus in capitulis mediocribus ovatis congestis, breviter pedicellatis subsessilibusi'e; calycis purpurascentis hirsuti ulta medium bilabiati dentibus porrectis albociliatis, lanii superioris lanceolatis. in Terioris subulatis: corollac purpureae tubo dentes calycis vix superante.

Stengel 3-10 $\mathrm{cm}$ lang; Blïtter $5-7 \mathrm{~mm}$ lang, $1 \mathrm{~mm}$ breit; Köpfchen $1 \mathrm{~cm}$ lang und ctwa cbenso hreit; kielch :3-4 mm lang.

Auf den höchsten Erhebungen des Peristeri, insbesundere in grosser Menge auf dem Gipfel. Höhe $2000-2290 \mathrm{~m}$.

Eine durch den dichtrasigen Wuchs, die sehr schmalen Blätter und dic zahlreichen gedrüingten hellpurpurnen Blüthenköpfchen auffüllige, wie es scheint auf den Hochgebirgen der Balkanhalbinsel unıl lileinasiens ziemlich verbreitete Art. In ihren Hauptmerkmalen und in der Tracht ist sie beständig, nur bezüglich der Beharung ist sie Abünderungen unterworfen, und es lassen sich diesbezüglich drei Varictïten, die allenfalls auch als Arten aufgefasst werden könnten, unterscheiden. Die erste Var. rividis, zu welcher auch die Pflanze rom l'eristeri gehört, hat zwar mit langen weissen Wimpern besetzte, aber sonst liahle Blätter. Die zweite Var. cilialopulescens hat ebenfalls langhewimperte Blätter, dic Flächen ler letzteren sind aber dicht mit äusserst kurzen Härchen bekleidet. Hieher gehört T. holosericens H. B raun apud Wettst. Beitr. Fl. Alban., p. 88 non Celak in Flora LXVI, p. 167. Endlich die dritte, Var. veslitus hat überall mit langen Haaren dicht besetzte Blätter.

Boissier erwähnt in der Fl. or. $1 \mathrm{~N}$, p. 557 von diesen Formen nichts und führt sie daselbst einfach unter dem Namen T. Lirsulus N. a Bieb. an. Dass dieser Name jedoch nicht für die hier besprochene Art verwendet werden kann, soll im Folgenden näher erörtert werden.

Welche Art M. a Bieberstein unter seinem T. hirsulus verstanden hat, lässt sich ohne Einsicht der Originalexemplare schwerlich ermittcln, es lässt sich nur aus seinen Beschreibungen und Citaten entnehmen, dass er bei Aufstellung seiner Art nicht die gleichnamige Pflanze Boissier's vor sich hatte. Am ehesten könnte noch die dritte obenerwähnte Varietät die Art Bieberstein repräsentiren, welche wenigstens mit der allerdings mangelhaften Diagnose in Fl. Taur. Cauc. II, p. 59 (1808) »loribus subcapitatis, caulibus procumbentibus, foliis linearisubulatis planis undique pilosissimis" in keinem Widerspruche steht. Birberstein citirt aber unter Anderen hiezu Serpyllum ramosissimum hirsutum angustifolium flosculis violaceis Ammann ruth. n. 68 und Serfyllum pannonicum tertium Clus. Hist. I, p. 360, deren Abbildungen sicher nicht unsere Pflanze darstellen; ferner T. foliis ellipticis hirsutis Hall. hist. n. 236, in welchem Werke wieder die Abbildung in Boccone's Museo di piante rare p. 108, t. 89, einer sicilianischen Pflanze citirt erscheint. Weiters sagt $\mathrm{M}$. a Bieb. a. a. O. "habitus et affinitas summa praecedentis varietatis $\hat{\beta}$ ", nämlich des T. Marschallianus, P anginstifolius procumbens. Nit T. Marschalliamus hat aber unsere Art auch sicher nichts gemein. Hiezu kommt noch, dass M. a Bieb. in Fl. Taur. Cauc Ill, p. 406 (1819) den T. pannonicus All. als Synonym zu seinem T. hirsutus zieht und als Standort "in saxosis ad monticulum qui thermas Constantinomontanis fundit desselben angibt, also einen niedriggelegenen, während unsere Art stets eine Pflanze der obcren Hochgebirgstegionen ist, welehe unter $1800 \mathrm{~m}$ nicht beobachtet wurde.

Diese angeführten Unstände beweisen wohl hinreichend, dass Bieberstein fast mit Gewissheit einer anderen und nicht der Boissier'schen Art den Namen T. hirsutus gab. Letztere musste daher auch mit einem neuen Namen belegt werden.

T. Boissicri steht in naher Verwandtschaft einerseits mit $T$. holosericulls Celak. I. c., andererseits mit T. cherlerioides Vis. 1hustr. di alc. piante della Grecia e dell' Asia minore, p. S (18+2). Ersterer, ron welchem mir ein von Heldreich auf dem Aenos in Cephalonien, dem Originalstandorte Čelakorsky's, gesammeltes instructives Exemplar vorliegt, unterscheidet sich ron ihm durch die spatelförmigen vder keilig-lanzettlichen spitzen Blätter, die Nervatur derselben, welche der cimptodromen schon wesentlich sich nähert und durch die grossen cilanzettlichen Bracteen. Des letzteren dlerkmales wegen gehört er in die Gruppe Heterolipides Borb., Wohin ihn auch schon Borbis a. a. O. gestellt hat.

ber in Vergessenheit gerathene $T$. cherlevioides Vis., welcher in neuerer Zeit von Člakovsky in Flora L.XY11, p. 534 (1884) unter dem Kamen T. humillimus neu beschrieben wurde, ist durch seinen 
äusserst dichten pulsterö̈migen Wuchs, die sehr kurzen, nur I-1·Jem langen Blüthenäste, die kleinen wenighlüthigen Köpfchen und die sitzenden kileinen Blüthen von $T$. Borssicri sehr leicht zu unterscheiden.

Mir beliannte Exemplare des T. Boissicri und zwar von Var. viridis: auf dem Olymp und Ossa in Thessalien (leg. Heldreich als T. hirsutus), auf dem Kossor-Gebirge in Macedonicn (leg. Dörlere) und aul" dem Peristeri in Epirus. Von V'ar. cilialopubesins: auf der liobilica im Scardus (leg. Dörfler als T. holosirieus) und auf dem Kudesi bei Vallona in Albanien (leg. Baldácci), auf der kiona in Nittelgriechenland, Wo ich sie im Jahre 1888 sammelte. Die dritte Varietät sah ich nur von den kleinasiatischen Gebirgen. ${ }^{2}$

351. Thymus capitatus L. Sp. pl. p. 568 sub Satureja (1753); Hoflm. et L.ink. Fl. Port. 1, p. 123 (1809).

In der Mediterranregion bei Arta. Höhe $50 \mathrm{~m}$.

352. Mentha Sieberi C. Koch in Linn. XX1, p. 649 (18+8).

In der Mediterranregion bei Alta und in der Bergregion bei Kalentini und Mazuki. Höhe $50-500 m$.

353. Mentha Pulegium 1. Sp. pl. p. 577 (1753). F. subtomentella H. Braun in Verh. zool. bot. Ges. Mien XL, p. 492 (1890).

In der Mediterranregion hei Arta. Höhe $50 \mathrm{~m}$.

\section{PRIMULACEAE I ent.}

35t. Lysimachia punctata L. Sp. pl. p. 147 (1753). Var. villicaulis nov. var.

Caules et petioli pilis longis mollibus dense villosis.

Der Stengel, der bei der typischen Form llaumig ist, weist bei der beschriebenen in seiner ganzen

Länge eine langharig-wollige Bekleidung auf.

In der Bergregion bei Kalentini. Höhe $400 \mathrm{~m}$.

355. Anagallis coerulea Schreb. Spic. Fl. Lips. p. 5 (1771).

In der Bergregion bei Vulgarelion. Höhe $600 \mathrm{~m}$.

356. Primula suaveolens Bert. in Desv. journ. bot. II, p. 76 (1813).

Auf dem Gipfel des Tsumerka mit Pidicularis gracca. Höhe $2336 \mathrm{~m}$.

\section{PLUMBAGINEAE Eind1.}

357. Armeria canescens Host Fl. Austr. 1, F.407 sub Statice (1827); Hust in Ebel Armer. gen. p. 2S $(1840)$.

In der 'Tannen- und angrenzenden obcren Reginn des T'sumerka bei Vulgarelion und Theodoriana. Höhe $1200-1500 \mathrm{~m}$.

Var. majellensis Boiss. in DC. Prodr. XII, p.685 pro spec. (1848). Conl. Beck Fl. Süibosn. I11, p. 127. Auf den Gipfel des Peristeri $(2290 \mathrm{~m})$ und des Tsumerka $(2336 \mathrm{~m})$.

\section{I.II. PLANTAGINEAE Juss.}

358. Plantago graeca Hal. in Verh. zoul. but. Ges. Mien, XXXY'lll, p. 761 (18s8).

In der oberen Region des Tsumerka und Peristeri, besonders am Rande der Schneefelder. Hölne "2000 $m$.

1 Einen ebenfalls in die Section der Hyphatromite gehörigen Thymus sammelte ich im Jahre $18 s 8$ in der oheren Region des Parnasses. Derselhe besizt holzige Stämmehen und treiht aus diesen rundum kurzhehatrte, dichtbeblätterte liangere sterile und chensolehe kïrzcre, 3-t cm lange blüthentragende. Iste. Die Blittel sind lineal, stumpf, sehr dicht, fast sammtartig kurzhaarig. zerstreut drüsig punkitut und am stark umgerollten Rand besonders gegen den Grund mit weissen Wimpern besetzt. Die Bracteen

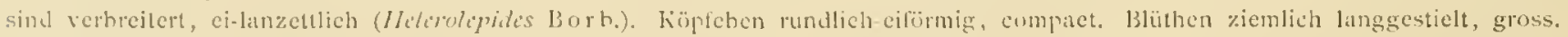
lielche dichtbehatrt, $4 m m$ ling, mit dichthewimperten Zähnen. Krone anschnlich, 7 mm liang, rosenroth, die Staubfïden mit ihren purpurnen Antheren aus ihr weit herastagend. Ich benenne diese sehüne Art $T$. purmasicus und bemerke, dass sie dem $T$. rato-

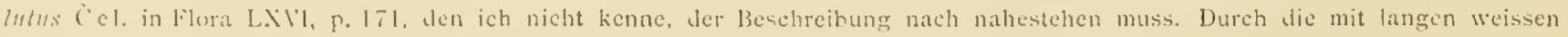
llaten dicht hesetzten Stengel und die breit rundlichen plötzlich zugespitzten brateten scheint dieser aber von $T$. farmassicus verschieden zul sein. 
359. Plantago lanceolata L. Sp. pl. p. 113 (1753).

In der Tamnenregion des Tsumerka bei Vulgarelion. Höhe $1000 \mathrm{~m}$.

360. Plantago Bellardi All. Fl. Ped. I, p. 82 (1785). P. pilosa Pourr. in act. lolus. 11t, p. 324 (1788). In der Nediterranregion bei Arta und Kalentini. Höhe $50-100 \mathrm{~m}$.

361. Plantago major L. Sp.pl. p. 112 (1753).

111 der 'lannenregion des Strungula bei Melisurgi. Höhe 1000 m.

362. Plantago brutia Ten. Prodr. Fl. Nap. p. LIX; Fl. Nap. 11I, p. 147, t. CXIII (1811-1815). Var. epirota. Folia integra, non ut in typo profunde dentata.

In der oberen Region des Tsumerka und des Strungula, seiten. Höhe $1800-2200 \mathrm{~m}$.

Ich führe diese Pflanze als Varietät von P. brulia Ten. an, weil sie mit Ausnahme der ganzrandigen Blätter, in allen Merkmalen mit dieser übereinstimmt. Yon der nächstverwandten $P$. media $L$. ist sie durch den kürzeren Stengel, die kurze eiförmige Ähre, spitze von einem grünen Mlittelstreifen durchzogene Kelchziplel und lanzettliche spitze Kronzipfel verschieden. Ihre Blätter sind stets sitzend, die Filamente nicht purpurn, sondern weiss.

\section{AMARANTACEAE Juss.}

363. Amaranthus deflexus L. Nant. p. 295 (1771).

Im Sande des Arachthos bei Arta. Höhe $50 \mathrm{~m}$.

\section{PHYTOLACCACEAE Endl.}

364. Phytolacca decandra L. Sp. pl. ed. 2, p. 631 (1762).

Im Sande des Arachthos bei Arta. Höhe $50 \mathrm{ml}$.

\section{SALSOLACEAE Mog. Tand.}

365. Blitum bonus Henricus L. Sp. pl. p. 218 sub Chenopodio (1753); Reichenb. Fil germ. cxc p. 582 (1832).

In der oberen Region des Tsumerka oberhaib Theodoriana. Hölı $2300 \mathrm{~m}$.

366. Chenopodium Botrys L. Sp. pl. p. 219 (1753).

Im Sande des Arachthos bei Arta. Höhe $50 \mathrm{~m}$.

LVI. POLYGONEAE JuSS.

367. Rumex crispus L. Sp. pl. p. 335 (1753).

In der Tannenregion znterhalb Kalarrytae. Höhe $600 \mathrm{~m}$.

368. Rumex conglomeratus Murr. Prodr. Stirp. Gotting. p. 52 (1770).

In der Tannenregion unterhalb Kalarrytac. Höhe $600 \mathrm{~m}$.

369. Rumex scutatus L. Sp. pl. p. 337 (1753).

In der Tannenregion $(600 \mathrm{~m})$ und in der oberen Region des Peristeri (2000 $\mathrm{m}$ ).

370. Rumex acetosella L. Sp. pl. p. 338 (1753). Var. multifidus L. Sp. pl. ed. 2. p. $48^{2} 2$ pro spec. (1762). In der Tannenregion unterhalb Kalarrytae. Höhe $600 \mathrm{~m}$.

371. Polygonum Convolvulus L. Sp. pl. p. 364 (1753).

In der Bergregion bei Vulgarelion. Höhe $800 \mathrm{~m}$.

372. Polygonum aviculare L. Sp. pl. p. 362 (1753).

In der Berg- und Tannenregion beiKalentini, Vulgarelion, Melisurgi und Kalarrtae. Höhe $500-1200 \mathrm{~m}$

LIII. THYMELEAE JUSS.

373. Daphne oleoides Schreb. Icon. et Descript. Fl. min. cogn. dec. I, p. 13 (1766).

In der Tannen- und oberen Region des Tsumerka. Strungula und Peristeri. Höhe 1000-1600 m. 


\section{I.VIII. LAURINEAE DC.}

374. Laurus nobilis L. Sp. pl. p. 369 (1753).

In der Bergregion bei Kalentini. Höhe $500 \mathrm{~m}$.

L.IX. SANTALACEAE R. Br.

375. Thesium Bergeri Zucc. PI. nov. II, p. 16. In der Bergregion bei Vulgatelion. Höhe $700 \mathrm{~m}$.

\section{EUPHORBIACEAE Juss.}

376. Euphorbia myrsinites L. Sp. pl. p. 461 (1753).

In der Tannen- und oberen Region des Tsumerka, Strungula und Peristeri schr häufig. Höhe 1000 bis $2200 \mathrm{~m}$.

377. Euphorbia herniariaefolia Willd. Sp. pl. 11, p. 902 (1799).

Auf dem Gipfel des Peristeri. Höhe $2196 \mathrm{~m}$.

378. Euphorbia helioscopia L. Sp. pl. p. 459 (1753).

In der Tannenregion bei Kalarrytae. Höhe $1000 \mathrm{~m}$.

\section{L.XI. PLATANACEAE Lind1.}

379. Platanus orientalis L. Sp. pl. p. 999 (1753).

In der Mlediterran- und Bergregion bei Kalentini, dann in der Tannenregion des Tsumerka bei Vulgarelion. Höhe $50-1200 \mathrm{~m}$.

\section{URTICACEAE Endl.}

380. Urtica dioica L. Sp. pl. p. $98+$ (1753).

In alten Regionen; steigt bis auf die Gipfel des Tsumerka und Peristeri. Höhe 50-2336 m.

381. Urtica pilulifera L. Sp. pl. p. 983 (1753).

Am Arachthos bei Arta. Höhe $50 \mathrm{~m}$.

\section{ULMACEAE Nirbel.}

382. Ulmus campestris L. Sp. pl. p. 225 (1753).

ln der Bergregion bei ǩalentini und Mazuki. Höhe $500 \mathrm{~m}$.

\section{J.XIV. CUPULIFERAE Rich.}

383. Quercus pubescens Willd. Sp. pl. IV, p. 450 (1805).

In der Bergregion bei Kalentini und Mazuki. Höhe $500 \mathrm{~m}$.

384. Quercus Ilex 1. Sp. pl. p. 995 (1753).

In der Bergregion bei Kalentini, Vulgarelion und Mazuki. Höhe $500 \mathrm{~m}$.

385. Quercus coccifera L. Sp. pl. p. 995 (1753).

In der Bergregion bei Kalentini und Mazuki. Höhe $600 \mathrm{~m}$.

386. Ostrya carpinifolia Scop. Fl. Carn. ed. 2, 11, p. 244 (1772).

In der Bergregion bei Kalentini, Mazuki und Kalarrytac. Höhe $500 \mathrm{~m}$.

387. Carpinus duinensis Scop. Fl. Carr. ed. 2, II, p. 243 (1772).

ln der Bergregion bei Kalentini und Mazuki. Höhe $600 \mathrm{~m}$.

\section{I.XYT. SALICINEAE Rich.}

388. Salix incana Schrank Baier. FI. I, p. 230 (1789).

In der Tannentegion des Tsumerka hei Vulgarelion. Höhe $1000 \mathrm{~m}$. 
380. Salix purpurea L. Sp. pl. p. 1017 (175:3).

In der Tannenregion des Tsumerlia bei Thendoriana. Höhe $1000 \mathrm{~m}$.

\section{ORCHIDEAE Juss.}

390. Epipactis latifolia L. Sp. pl. p. 949 pro rar. Serapludis Helleborines (1753); Alt. Fl. Ped. II. P. $15(1785)$.

In der Tannenregion des Tsumerka wherhałh Vulgarelion; sehr selten. Höhe $1200 \mathrm{~m}$.

301. Epipactis microphylla Ehth. Beitr. IV, p. t2 sub Surapiade (1792); Sw. in Vel. Icad. Handh. p. $2932(1800)$.

In der Tannenregion des T'sumerka oberhalb V'ulgarelion, sehr seltun. Höhe $1200 \mathrm{~m}$

392. Anacamptis pyramidalis L. Sp. pl. p. 940 sub Orchide (175:3); Rich. Orchic. Europ. Annot. p. 33 (1817).

In der Tannenregion des Strungula oberhalb Theodoriana. Höhe $1200 \mathrm{~m}$.

\section{L.XVII. IRIDACEAE Lindl.}

393. Crocus veluchensis Herb. in Edwards Bot. Register XXXI, Mise. P. Su (1845) et 1. c. XXXIll, t. t. l. $3(18+7)$.

An Schnecfeldern in der oberen Region des Tsumerka und Peristeri. Hühe $2000 \mathrm{~m}$.

\section{LILIACEAE DC.}

39. Lilium candidum L. Sp. pl. p. 302 (1753).

An schwer zugänglichen felswänden in der Tannenregion bei kalarrytat. Höhe $1000 \mathrm{~m}$. Wh sethst sah nur die von hier mitgebrachten cultivirten Exemplare.

395. Fritillaria messanensis Raf. Préc. des découv. p. tt (1800).

In der oberen Region des Tsumerka uberhalb Thoodoriana, schr selten. Höhe 2000 m.

396. Asphodeline lutea L. Sp. pl. p. 309 sub Asphodelo (1753); Reichenb. Fi. Germ. exc. p. HIf $(1830)$.

In der Tannenregion des Peristeri bei Kalarrytac und Chaliki. Höhe $1000 \mathrm{~m}$.

397. Scilla bifolia L. Sp. pl. p. 309 (1753). Var. nivalis Boiss. Diagn. Pl. or. Sor. 1, Nr. 5, p. 63 pro spec. $(1 S+4)$.

An Schneefeldern in der oberen Region des Tsumerka oberhatb Theodoriana mit Crocus aiduchensis. Höhe $2000 \mathrm{~m}$.

398. Allium atroviolaceum Boiss. Diagn. P1. or. Ser. I, Nr. 7. P. 11 (18.16).

In der oberen Region des Peristeri bei Kalarrytac. Höhe $1200 \mathrm{~m}$.

399. Allium margaritaceum Sibth. et Sm. Fl. Graec. Prodr. I, p. 22- (1806).

In der Mediterranregion bei Kalentini. Höhc $100 \mathrm{~m}$.

400. Smilax aspera L. Sp. pl. p. 1028 (1753).

In der Mediterranregion bei lialentini. Höhe $100 \mathrm{~m}$.

\section{LAIX. COLCHICACEAE DC.}

401. Veratrum Lobelianum Bernh. in Schrad. Neu. Journ. f. Bot. 11, p. 356 (1807)

In der oberen Region des Peristeri, truppenweise. Höhe $2000 \mathrm{~m}$.

\section{LXY. ARACEAE Meisn.}

102. Arum italicum Mith. Dict. cd. 8, Nr.2(1768).

In der Tannenregion bei Mazuki und in der oberen Region des Puristeri bei kalarrytae. Hohe $1300 \mathrm{~m}$. 


\section{L.XXI. CYPERACEAE JUSS.}

403. Cyperus longus I. Sp. pl. p. 5. (1753). Var. pallidus Boiss. Fil. or. V. p. 375 (IS84). Im Sinde des Arachthos bei Artil. Höhe $50 \mathrm{~m}$.

40.4. Carex laevis Kit. in Willd. Sp. pl. IT, p. 292 (ISO5).

In der oberen Region des Strungula uberhalb Melisurgi. Höhe $1800 \mathrm{~m}$.

\section{GRAMINEAE JUSS.}

40.5. Andropogon Gryllus L. Am. IV, p. $3: 32$ (1750).

In der Mediterranregion bei Kalentini. Höhe $100 \mathrm{~m}$.

to6. Phleum pratense L. Sp. pl. p. 59 (1753).

In der Tannenregion des Tsumerka bei Vulgarelion. Höhe $900 \mathrm{~m}$.

407. Phleum commutatum Gaud. Agrost. helv. I, p. 40 (1811).

In der oheren Region des Tsumerka und Peristeri. Höhe $1800 \mathrm{~m}$.

408. Alopecurus Gerardi All. 11. Peden. II, p.232 sub Phlen (1785); V'ill. pl. Dauph. IJ, p.66 (1787). In der oberen Region des Tsumerka oberhalb Theodoriana. Höhe $2200 \mathrm{~m}$.

409. Sesleria coerulans Friv. in Florn XIX, p. 438 (1836). - S. marginata Griseb. Spic. Fl. Rum. et Bithyn. 1, p. 142 (1844). - S. Bivlaii Schur in Verhand. Siebenb. Ver. 1, p. 109 (1850). - S. vaginalis Boiss. et Orph. Diagn. Pl. or. Ser. II, Nr. 4, p. 130 (I 859 ).

In der oberen Region des Strungula oberhalb Nelisurgi. Höhe $1800 \mathrm{~m}$.

4 I0. Cynosurus echinatus L. Sp. pl. p. 72 (1753).

Gemein in der Nediterran-, Berg- und Tannenregion von Kalentini über Vulgarelion, Mazuki bis lialarritae. Hölue $50--$ - $500 \mathrm{~m}$.

411. Agrostis vulgaris With. Arr. p. 132 (1776).

In der Bergregion bei Kalentini. Höhe $500 \mathrm{~m}$.

412. Stipa pennata L. Sp. pl. p. 78 (1753).

In der oberen Region des Peristeri oherhalb Kalarrytae. Höhc $1210 \mathrm{~m}$.

413. Holcus lanatus L. Sp. pl. p. 1048 (1753).

In der Bergregion bei Kalentini. Höhe $500 \mathrm{~m}$.

414. Trisetum flavescens J. Sp. pl. p. 80 sub Arena (1753); P. de Bcauv. Agrost. p. 88 (I812).

In der oberen Region des Tsumerka oberhalb Theodoriana. Höhe gon $\mathrm{m}$.

415. Aira capillaris Host Icon. ct Descript. Gram. Austr. IV, p. 20 (ISO5).

In der Tannenregion des Tsumerka oberhalb Vulgarelion. Höhe $1000 \mathrm{~m}$.

116. Koeleria splendens Presl Cyp. et Gram. sic. p. 34 (1820). - K. grandiflora Bert. in Schult. Mant. 11, p. 345 (1824).

In der Tannenregion des Tsumerka bei Vulgarelion. Höhe $1000 \mathrm{~m}$.

417. Melica ciliata L. Sp. pl. p. 136 (1753).

In der oberen Region des Tsumerka bei Theodoriana und des Peristeri bei Syraku, dann in der Tamenregion bei Mazuki. Höhe $600-1200 \mathrm{~m}$.

418. Dactylis glomerata L. Sp. pl. p. 7 I (1753).

In ler Bergregion bei Kälentini und Mazuki. Höhe $500 \mathrm{~m}$.

419. Bromus squarrosus J. Sp. pl. P. T6 (175\%).

In der obcren Region des 'Tsumerka oberhath Theodoriana. Höhe $1300 \mathrm{~m}$.

420. Bromus patulus Mert. et kuch in Röhl Deutschl. Fl. 1, p. 685 (1823).

In der Tamnenregion des Tsumerka bei l'ulgarelion. Höhe $1000 \mathrm{~m}$. 
421. Festuca elatior L. Sp. pl. p. $75(1753)$.

In der 'lannenresgion des 'l'sumerka bei Vulgareliom. Höhe $1000 \mathrm{~m}$.

122. Festuca acuminata Gaud. Agrost. helv. Il, p. 287 (1811). - F. flncescens Ciaud. I. p. 272 (non Bell.).

Auf dem Gipfel des Peristeri. Höhe $2290 \mathrm{~m}$.

423. Festuca sulcata 11 a cliel Nonogr. Festuc. Europ. p. 104 pro sulpspec. F. orinat (J889). Val. saxatilis Schur Enum. pl. Trans. p. 791 pro spec. (1866): Hackel 1. c. p. 105 pro stibrill. F. orinut.

Auf dem Gipfel des Peristeri die ganzen Abhänge hedeckind (2') $(0) \mathrm{m}$ ).

424. Festuca valesiaca Schleich. in Gaud. Agrost. helv. 1, P. 2.12 (1811).

In del oberen Region des T'sumerlia. Höhe $1000 \mathrm{~m}$.

425. Briza elatior Sibth. et Sm. Fl. Graec. Prodr. I, p. 57 (1806).

In der oberen Region des Tsumerka bei Theodoriana. Höhe 11$)(10 \mathrm{~m}$.

420. Poa alpina L. Sp. pl. p. 07 (1753). Var. parnassica Boiss. Fil. (14. 1', p. 605) (1854).

In der oberen Region des Tsumerka und Peristeri. Höhe $2000-2330 \mathrm{~m}$.

427. Poa violacea Bell. App. ad Fl. ped. P. 8 (1792).

In der oberen Region des Tsumerka oberhalb Theodoriana. Höhe $2000 \mathrm{~m}$

428. Poa Timoleontis Heldr. in Boiss. Fl. or. V, p. 607 (1SS4).

In der oberen Region des Tsumerka oberhalb Theodoriana. Höhe $2000 \mathrm{~m}$.

429. Poa annua L. Sp. pl. p. 68 (1753).

In der Tannenregion des Strungula bei Nelisurgi. Höhe $1000 \mathrm{~m}$.

430. Hordeum bulbosum I. Am. acad. IV, p. 304 (1750).

In der Bergregion bei Vulgarelion. Höhe $700 \mathrm{~m}$.

431. Hordeum murinum L. Sp. pl. p. 767 (1753).

In der Bergregion bei Kalentini und in der Tannenregion bei Melisurgi. Höhe $500-900 \mathrm{~m}$.

432. Haynaldia villosa L. Sp. pl. p. St sub Secale (1753): Schul Enum. Trans. p. S07 (1866).

Von der Mediterranregion über die Berg- und Tannenregion von Kálentini über V'ulgarelion, Mlazuki bis Kalarytae, selbst noch in der oberen Region des Tsumerka und Peristeri. Höhe .70-1:300 m.

433. Brachypodium distachyon L. Am. Acad. IV, p. 304 sub Bromo (1759); Roem. et Schult. Syst. Il, p. 74 (1817).

In der Tannenregion des Tsumerka oberhalb Vulgarelion. Höle $1200 \mathrm{~m}$

434. Lolium perenne L. Sp. pl. p. 83 (1753).

In der Tamnenregion des 'Tsumerka oberhalb Vulgarelion. Höhe $1000 \mathrm{~m}$

\section{CONIFERAE JUSS.}

435. Abies Apollinis Link. in Linn. XY, p. 528 (1841).

Als ausgedehnter Bestand die Tamnenregion auf dem Tsumerka, Strungula und Peristeri hildend. Die untere Grenze desselhen wurde auf dem Tsumerka bei Vulgareliun in (1stexposition mit Ttom, bei Theodoriana in Nordostexposition mit $910 \mathrm{~m}$. die obere Grenze ïher dem letztgenannten Orte nit $1: 390 \mathrm{~m}$ bestimmt. Die obere Grenze auf dem Peristeri bej Kalarytae in Südexposition 11 f() $m$.

436. Juniperus Oxycedrus L. Sp. pl. P. 1038 (1753).

In der Berg- und Tannenregion des Tsumerka und Peristeri und in der angrenzenden oberen Region Höhe $700-1200 \mathrm{~m}$.

437. Juniperus foetidissima Willd. Sp. pl. 11, p. $5.33(1805)$.

In der Tamnenregion des Tsumerlá oberhalb V́ligarelion, selten. Höhe $1100 \mathrm{~m}$. 


\section{EQUISETACEAE DC.}

438. Equsetum palustre L. Sp. pl. p. 1061 (1753).

In der oberen Region des T'sumerka bei Theodoriana. Höhe $1100 \mathrm{~m}$.

\section{FILICES JuSS.}

139. Ceterach officinarum IVilld. Sp. pl. V, p. 136 (1810).

In der Pergregion bei Lalentini. Höhe $500 \mathrm{~m}$.

440. Pteris aquilina L. Sp. Pl. P. 1075 (1753).

C̈berall in der Berg-, Tannen- und angrenzenden oberen Region, stellenweise ausgedehnte dichte Bestiinde bildend. Höhe $500-1200 \mathrm{~m}$.

441. Adianthum capillus Veneris L. Sp. pl. p. 1090 (1753).

In der Bergregion bei Kalentini. Höhe $600 \mathrm{~m}$.

442. Asplenium Trichomanes L. Sp. pl. p. 1080 (1653).

In der Bergregion bei Kalentini. Höhe $600 \mathrm{~m}$.

443. Asplenium Virgilii Bory in Exp. scient. de Norée 111, 2, p. 289 (1832).

In der Rergregion bei kalentini. Hohe $6(0) \mathrm{m}$.

44. Aspidium Filix mas L. Sp. pl. p. 1090 sub Polypodio (1753); Sw. Cien. ct spec. Filic. in Schrad. Journ. 1800, 1I, T. 29.

In der Bergregion bei lialentini. Höhe $600 \mathrm{~m}$.

445. Cystopteris fragilis L. Sp. pl. p. 1091 sub Polypodio (1753); Döll Khein. Fl. p. 15 (1843). In der oberen Region des Peristeri. Höhe $1800 \mathrm{~m}$.

\section{Moose.}

446. Leptotrichum flexicaule Schleich. Plant. crypt. helr. Cent. +, Nr.9 sub Didymodonte (1807); Hampe in Linnaea XX, p. $7+(18+5)$.

Spürlich zwischen Reboulia hemisphacrica, steril. Auf dem Tsumerka.

47. Didymodon rubellus Holfm. Deutschl. Fl. 11, p. 33 sub Bryo (1796); Bryol. eur. Il, t. 185 (1846). Sparlich mit Rebonlia homisplatira, c. lruct. Auf dem 'Tsumerka.

448. Didymodon luridus Hornsch. in Spreng. Syst. IV, I, p. 173 (1827): Bryol. eur. 11, t. 186 (1846). Pei Kulentini, auf Plalanus c. fruct.

449. Barbula unguiculata Huds. 1.1. angl. cd. I, p. 410 sub Bryo (1702); Hedw. Fund. II, p.92 (1782). Bei Kälentini, auf Platanus c. fruct.

450. Barbula tortuosa L. Sp. pl. p. 1119 sub Bryo (1753); Web. et Mohr Bot. Taschenb. p. 285) (1807). Auf dem Tsumerka, c. fruct.

4.7. Barbula subulata L. Sp. pl. p. 1116 sub Bryo (175:3); P. de Beauv. Prodr. p. 43 (1805). Bei Kalentini, auf Plahmus c. liuct. und aul dem Tsumerka, zwischen Reboulia homispharica c. fruct. 152. Barbula ruralis L. Sp. pl. p. 1110 sut Bryo (1753); lied I. Fund. Il, p. 92 (1782).

Auf dem Tsumerka, mit Reconlia hemisplucrica, steril.

45\%. Grimmia apocarpa L. Sp. 1. P. 1115 sub Bryo (1753); Hedw. Descr. 1, p. 104, t. 39 (1787). Bei Vulgarclion, c. fruct.

454. Grimmia pulvinata L. Sp. pl. p. 1121 sub Bryo (1753); Smith Engl. Bot. t. 1728 (1807). Bei l'ulgarelion, c. liruct. 


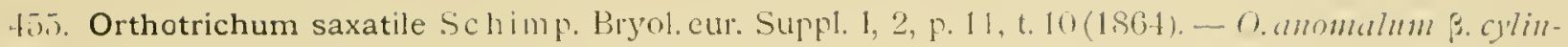
dricum Schimp. Sy"n. ed. 2, p. 309 (1876).

Bei Vulgarelion, c. fruct.

456. Orthotrichum Sardagnanum Vent. Rer. bryol. V1, p. 56 (187!)). - (). Sarlagnue V'ent. in 11 usnot Mluscol. gall. p. 161, t. 45 (1887).

Bei Vulgarclion, c. fruct.

457. Orthotrichum fastigiatum $\mathrm{Bruch}$ in Brid. BrynI. univ. I, p. 78.5 (1826).

Mit Hobrodon Sotarisii, c. fruct. Bei Pramanta.

458. Orthotrichum speciosum Nees in Sturm Deutschl. Fi. Crypt. fasc. 17 (1819).

Spärlich mit roriger Art, c. fruct. Bei Pramanta.

45!). Orthotrichum pumilum Ser. Disp. musc. suec. p. 42 et 92, t. 4 , f. (1) (1798).

Mit den vorigen spärlich, c. fruct. Bei Pramanta.

460. Orthotrichum Lyellii Hook et 'T'ay 1. Nusc. brit. p. 70. t. 22 (1818).

Spürlich mit vorigen, steril. Bei Pramanta.

461. Orthotrichum striatum L. Sp. pl. p. 1115 sub Bryo (175:3); Schwägr. Suppl. 1, 2, p. 29, t. 51 (1S16). - Orthotrichmm leiocarmm Bryol. eur. 11], t. 230 (1837).

Spärlich mit vorigen, c. fruct. Bei Pramanta.

462. Funaria hygrometrica L. Sp. pl. p. 1110 sub Mnio (1753); Sibth. Fl. Oxon, P. 288 (1794); Hedw. Spec. musc. p. 172 (1801).

Bei kalarrytae, c. fruct.

403. Bryum caespititium L. Sp. pl. p. 1121 (1753).

Zwischen Funaria lygromelrica, steril, +. Bei Kalarytae.

464. Bryum pseudotriquetrum IJ edw. Descr. JII, p. 19, t. 7, ex p. sub Mnin (1792); Schwäigl. Suppl. 1. 11, p. 110 excl. Syn. B. bimm (1817); Bryol. eur. IV, t. 364 (1839).

Mit Plitonolis fonlana, steril, $f$. Auf dem Peristeri.

465. Bryum Schleicheri Schwägr. Suppl. I, II, p. 113 (1816). Var. latifolimm Schimp. Syr. ed. 2, P. 46:3 (1876). - Mnimm latifolimm Schleich. Cat. 1807; Schwïg1. Suppl. 1, 11, p. 138 (1816).

Zwischen Plibonotis calcarea, steril. Auf dem Peristeri.

466. Philonotis fontana L. Sp. pl. ed. 2, p. 1574 sub Mnin (1763); Brid. Bryol. univ. 11, p. 18 (1827). Auf dem Peristeri, c. fruct. et pl. $o^{\nearrow}$.

467. Philonotis calcarea Schimp. Coroll. p. S6 (18.56). - Fiatramia calcarea Bryol. eur. IV, t. 325 $(18,7)$.

Auf dem Peristeri, c. fluct. et pl. $\sigma^{\top}$.

468. Leptodon Smithii Dicks. Fasc. pl. crypt. II, F. 10, t. 5, f. 4 suh Hypro (17(10)): Mohr Obs. hot. p. $27(180: 3)$.

Spärlich mit Habrodon Notarisii, steril. Bei Pramanta.

469. Pterogonium gracile L. Syst. leg. ed. 13, p. 801 sub llypnn (177-1); Sw. Disp. musc. suec. p. ¿26 (1798).

Bei Kalentini, auf Platamms, c. fruct.

470. Habrodon Notarisii Schimp. Syr. cd. 1, p. 505 (1860).

Bei Pramanta, c. fruct.

171. Anomodon viticulosus L. Sp. pl. P. 1127 sub Hypno (1753); Hook. ct Tay I. Musc. Brit. P. T9, t. 2'- (1818).

Mit Pterogonimm gracile, auf Platamus, steril. Bei Kalentini. 
472. Pseudoleskea atrovirens Dicks. Bryol. eur. $\mathrm{V}$, t. 477. - Hypum atrotirans Sim. Fingl. bot. t. $2+2$ '2.

Spïrlich zwisehen Barbula lortnosa, sterit. Auf dem Tsumerka.

473. Homalothecium sericeum L. Sp. p. p. 1129 sub Hypno (175:3); Bryol. eur. V, t. 450.

Bei Kalentini, c. lruct.

47. Scleropodium illecebrum Schwägr. Suppl. I, 11, p. 225 suh Hypno (1816); Bryol. cur. V', t.557. Spärlich mit Barbula subulata, aul Platanus, steril. Bei Kalentini.

475. Hypnum commutatum Hedw. Descr. IV. P. 68, t. 20 (1794).

Spärlich mit Philonolis calcarca, steril. Auf dem Peristeri.

47t. Hypnum cupressiforme L. Sp. pl. p. 1129 (1753).

Spärlich zwisehen Homalolhccinm scriccum, steril. Bei lialentini.

47. Radula complanata Dum. Reeueil d'obs. p. 14 (183.i).

Auf Plalanms, e. llor. + et $\sigma^{7}$. Bei kialentini.

478. Frullania dilatata L. Sp. pl p. 1133 sub Jungermannia (1753); Dum. Recueil d'obs. p. 13 (1835). Sparlich mit voriger Art, nuf Platanus. steril. Bei líalentini.

47. Reboulia haemisphaerica L. Sp. Fl. p. 1138 sub Marchantia (1753): Raddi in Opusc. scient. Bologna 11, p. 357 (1818).

Auf dem Tsumerka, e. fruct.

\section{Flechten.}

Das Suhstrat der unten genannten Flechten bilden Kalke, grauer und rother derber Feldspath rom Peristeri, kalk von Tsumerlia, Kalk und Mergel von kalatrytae, Rinde von Ilulamms und Walderde von Kalentini.

480. Physcia caesia Nyl. Prodr. p. 308. - Hoffm. En. p. 65 sub Lichone, rar. albinea Th. Fr. aret.

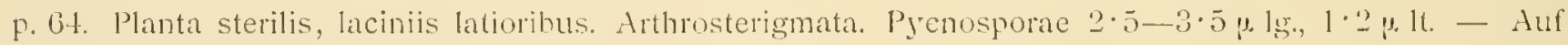
Kalle der Peristeri-Spitze.

481. Rinodina immersa Arn. Jur. Sep. p. 104. - Fib. Par. p. 75, K. Bischofii B immorsa. - Die normale Pllanze auf Kalk des Peristeri und von Kalarrytac.

482. Sarcogyne pusilla Anzi Man. in Comm. er. it. I (Genova), p. 157 exs. Loy. 190.

Apothecien kleiner als hei Arn. Exs. 465. - Auf kalk des Peristeri.

483. Caloplaca (Sect. Amplitoma) elegans Th. Fr. Sc. p. 168. - Link Ann. d. Bot. 1, p. 37 - F. compacla Arn. Flur. 1875, p. 151. - Auf Kalk der Peristeri-Spitze.

484. Caloplaca (Sect. Amplitoma) murorum Th. Fr. Sc. p. 170. - Hoffm. En. p. 63 sub Lichenc. -

Aul Kalk der Peristeri-Spitze; aul Nergel von Kalarrytae mit Nr. 36.

485. Caloplaca aurantiaca Th. Fr. Sc. p. 177. - Lightf. Flor. Scot. II, p. 810 sub Lichene. - Sporac $16-18 \% 1 \mathrm{~g} ., 7-11 \%$. $1 \mathrm{t}$. Thallus depauperatus formatur granulis parvis albis (ad latera saxi luteo aut viridulo albis) dissipatis aut congestis. Afothecia emergentia normalia majora, hic inde mutua pressione angulosa. - Auf kialk der Peristeri-Spitzc.

486. Caloplaca (Sect. Pyrenedesmia) chalybaea Th. Fi. Sc. p. 172. - Duf. in E. Fr. L. eur. p. 125. planta nomalis. Apothecia madida fuscescentia. - Aul Kalk der Peristeri-Spitze und von Kalarytac.

\footnotetext{
1 lícarbeitul von J, Stcincr.
} 
187. Caloplaca (Sect. Pyoundesmil) intercedens Sitzungsh. d. k. Mkad. d. Wiss. Wien, Bd. C11, Ihth. 1,

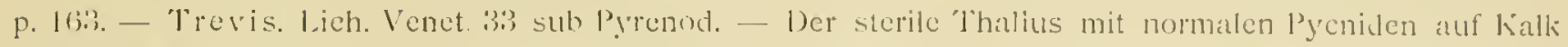
des Peristeri. Aul einem zweiten Stüclic Källi einige Apothecien.

Var. allomarsinata m.

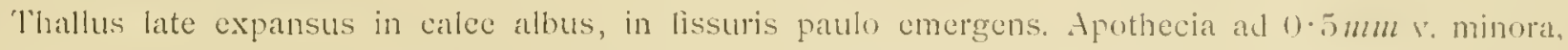
primum immersa deinde adpresse convexiuseula, margine thallode albo, margine proprio nom $r$ parum croluto. Sub hypothecio gonidia adsunt. Sporae $14-20 \%$ Ig. S-10\%. It. Pycnides et pycnospolale normales. - Die Varietät gehört zu derjenigen Formenreihe der inloredens, welche wenigstens im Anfange deutlich dem Kalke eingesenkte Apothecien besitzt und ist in diescre dureh den Apothecienrand und in zweiter Linie durch etwas grössere Sporen gekennzeichnet. Übergänge sind entschieden vorhanden. So kimn e. c. das untere Exemplar ron Hepp exs. tö (Herb. Univ. Vienn.) als Zwischenlinm aufgelisst Werden. - Auf mehreren Stïcken Kalk vom Peristeri reich vertreten.

488. Caloplaca (Sect. Pyrenodesmiu) fulva Mïll. Arg. Flor. 1872, p. 470. - Anzi Symb. p. 7 sul Zeora. Dieselbe Pflanze wie rom Cap Sunion; comp. Sitzbungs. d. k. Akad. d. Miss. Mien 1. c., p. Mij.

489. Caloplaca (Sect. Mhastcria) ferruginea Th. Fr Sc. p. 182. - Huds. Fl. Angl. ed. 11, p. 526 sub Lichene. - Apothecia parva (ad $0.4 \mathrm{~mm}$ ) rufa, nitidiuscula super crustam depauperatam, tenuissiman, cinereo-albidam. - Auf lialk del Peristeri-Spitze.

490. Caloplaca (Sect. Pyrenodesmia) variabilis Th. Fr. Scand. p. 172. - Pers. in Ust. Ann. p. 26 sub Lichene.

Thallus areoliert, braunlich grau. Pyeniden normal. - Auf den Areolen Arth. vaguns var. Körb?ri und Lecania Rubinhorstiif. - Leg. Hartl.

401. Gyalolechia (Sect. Candclaria) vitellina Th. Fr. Spitz. p. 10. - Ehrh. Plant. eryp p. $155 .-$ Auf Feldspat der Peristeri-Spitze neben und zum Theile über Rhiz. geograph.

492. Gyalolechia vitellinellum Mudd Man. of Brit. -- Lich. 135 sub Callopis. — Auf dem Kalli les Peristeri. Leg. Hartl.

493. Lecanora (Sect. Placodium) crassa Ach. L. Univ. 1. H13. - Huds. Fl. Angl. 11, p. 530 sub Lichene. - In den Ritzen des Kalkes der Peristeri-Spitze.

494. Lecanora (Sect. Placolinm) muralis Sehär. En. 4. - Arn. Jur. Sep. 97 sub Placod. — Schreb. Spic. p. 130. - Auf Feldspat der Peristeri-Spitze. Dabei eine Form, welche zu Lec. diffracta Ach. L. Unis. p. 432. - Nyl. Scand. p. 133 gezogen werden kann.

Var. rersicolor Pers. in Ust. Ann. Bot. 1794. p. 24. - F. subsulphmrata. - A versicolore differt colore thalli viride sulphureo ad marginem laciniosum et hic inde tantum ad partes adultiores pruina levi dealbato. Pycnosporae longiores 28 - 35 \% $\mathrm{lg}$. arcuatae raro subflexuosae sterigmatibus brevibus affixac. - Auf lialk von Kalarytae und des Peristeri, theilweise den Thallus ron L. Hartliana hodeckend. Leg Hartl. Dic sehr zierliche Pflanze gleicht in der Farbe unbereiften Exemplaren der Loc. saxicola aus der Herzeguwina (leg. Loyka) und von Jowa (leg. Ehrfeld) im Herb. des k. Hofmuscums in Wien. Andererscits steht ihr eine rersicolor rom Karst in Herb. Eggerth sehr nahe.

495. Lecanora (Sect. Placodinm) subdiscrepans Nyl. Flor. 1861, p. T18, cxs. Loyka Hung. 25 , comp., Sitzb. Helv. p. S7. - Steril auf Feldspat der Peristeri-Spitze.

496. Lecanora (Sect. Placodimm) circinata Ach. Univ. p.425. - Pers. in Ust. Ann. 7. p. 25 sub Lichene.

Thallus KHO non mutatus, depauperatus, minus distincte laciniatus. - Auf Feldspat der PeristeriSpitze.

497. Lecanora dispersa Flk. D. Fl. 11, p. 4. - Pers. in Ust. Ann. 7, p. 27 sub Lichene. - Auf Kálk der Peristeri-Spitze.

198. Lecanora crenulata Nyl. Flor. 1872, p. 250.-- Dicks. Crypt. 111. p. 14. Syn.: Lec. caesioalha lirh. Par. p. 82. - Auf Kalk der Peristeri-Spitze und auf Nergel von lialímytae. 
490. Lecanora (Sect. Aspicilia) calcarea Som.

Var. concrela Schär. Spic. 73. - Auf Kalk der Peristeri-Spitze und aul Kalk von kalarrytae.

500. Lecanora (Sect. Aspicilia) farinosa Nyl. Flor. 1878. p. 2248. - Flk. in Bcrl. Mag. 1810 p. $125 .-$ Auf Kalk der Peristeri-Spitze.

501. Lecanora (Sect. Aspicilia) Hartliana m.

Thallus medioc. crassus $r$. crassus, cinereo-albus aut argillaceo-albus, alhopruinosus r: loco obumbrato sublaevis, madefactus caesio-cinereus; centroversus areolatus, areolis fertilibus convenis; ad ambitum suborbiculare diteminatus, lobatus. Lohi breviores, lineares, conniventes, digitato incisi, convexi al marginem fusciduli. Apotheciorum (ad $0.5 \mathrm{~mm}$ ) structura cadem ac minorum lacvatac Nyl.. at margo et discus albopruinosus et sporac minores et p. m. latiores $13-20 \% 1 \mathrm{~g}$. 10-14 $\mu$ lt. Pycnides supra atrae, simplicis aut plurilocalares, saepius plures in quaris areola congestae pseudodiscum interuptum formantes (simili modo ac in Asp. amphihola Norrl. Fèn. exs. 243). Sterigmata subsimplicia, ramosa, pycnospurae subrectac, arcuatac aut flexuosae $18-28$ raro $30 \mu \mathrm{lg} .1-1.5 \mu \mathrm{lt}$. - In mehreren theils zusammenfliessenden, theils getrennten Rosetten auf dem kalk des Peristeri (leg. Hartl).

Die lilechte schliesst sich der Lecau. lacvala Nyl. (Ach.) mit den ff. supertegens Arn. exs. 60 s und alficans Arn. exs. 1167 nahe an, ist aber besonders durch den Thallus, doch auch dureh die bereiften Apothecien, die kleineren Sporen und die um etwas grösseren Pycnosporen verschieden. Asp. ciucrea, f. cliodectonoidcs Anzi, Welche Stitzenberger Helv. p. 126 zu laczala zieht, Wonach anzunehmen ist, dass die Pyenosporen entsprechen, hat nach Anzi Neosymb. p. 7 jedenfalls einen ganz anderen Thallus und Apothecia 2-12 confluentia." Aspic. polyclurona, f. candida Anzi Cat. 59 und cxs. 1g. 325, It. sup. 208 hat zwar spurweise effigurirtes Lager, wie es ja in dieser Gruppe nicht selten ist und ebenfalls kiteinere Sporen; aber diese Effiguration ist ron den deutlichen convesen Lappen der Harlliana, auch abgesehen von der Farbe, bedeutend verschieden, und die Pycnosporen sind entschieden kleiner 12-20 j. $1 \mathrm{~g}$., so dass candida Anzi, wie auch Stitzenberger Hels. p. 120 annimmt, als eigene Art zu betrachten ist. An der schönen Flechte Arn. exs. 999 vermag ich keine Pyeniden zu finden. Asp. polychroma Anzi lat. 59 exs. Ig. 277 ist schon durch die Pycnosporen deutlich getrennt. Diese sind wie bei lacvala, $18-25 \mu$.g., aher weitaus vorherrschend gerade, oder so leicht gekrümmt, dass ihr Typus als der gerade betrachtet werien muss. Jch habe noch zu bemerken, dass bei candida Anzi, polychroma Anzi und polychroma f. pallescons Anzi, Lang. 530 die Paraphysen immer auffatlend zellig getheilt sind, was, soweit mir bekannt, weder bei lactala noch Harlliana der Fall ist.

502. Lecania (Sect. Dincrospora) Rabenhorstii Arn. Jur. no. 201. - Hepp. Eur. n. 75. - Auf Mergel bei Kalarrytae sehr wenig entwickeit. - F. slutroplica. - Apothecia subbiatorina tandem convexiuscula, obscure fusca, madida dihutiora epithecio obscure cerasino v, fusco-cerasino, paraphysibus supra incrassatis et septatis. Sporae 8 oblongae rectac $v$ leviter currulae $12-17 \% \lg .5-7 \mu \mathrm{H}$. E Einige Apothecien sitzen fast unmittelbar neben denen der Arth. Kürbori auf den Areolen der Cal, tariabilis, welche merktich an Dicke zugenommen haben, andere scheinen eigene Arcolen zu besitzen, welehe aber mit denen der z'ariabilis so verwachsen sind, dass cine sichere Trennung nicht möglich ist. Für weitere Untersuchung ist das Material zu beschränkt. Die systematische Stellung der Flechte bleibt unsicher, sie wurde der interessanten hiologischen Verhältnisse wegen angeführt.

503. Ricasolia amplissima Leight. Lech. Ft. p. 112. - Scop. F1. Can. ed. 2, 1772, p. 386 suh l.ichene. - Arthrosterigmata. Pyenosporae rectae al $5 \mu$ lg. 1 \%. It. medio leviter constrictae. - Aul Platanenrinde von Kalentini.

504. Pannaria triptophylla Krl. Syst. p. 107, 1855. - Nyl. Prodr. p. 67, 1857. - Ach. L. Univ. p. 215. Apothecia parva rula 1 rufo-fusca. - Neben Ricasolia amplissima.

505. Synechoblastus nigrescens Trev. Chatratt. di 3 11. gen. d. Lollem. 1853. - Huds. F1. Angl. p. 150. Steril neben Catillaria grossa auf Platanenrinde von kalentini. 


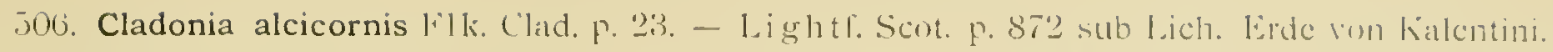

507. Bacidia rosella 1). Not. Framm. lich. p. 190. - Pers. in Ust. Ann. 7. p. 75 suh 1.ich. Nur zwes Apothecien neben Ricasolia amplissima.

508. Lecidea rhaetica Krb. Par. p. 207. - 11epp. in lit. ad Krh. suh Biatora. - Arn. cxs. 117.

Thallus hic inde I coerulescit (comp. Arn. in Verh. d. \%. hot. (ies. Wien 1876, p. 366). Sporac $20-26$ j. lg. $10-13$ \%. ll. Auf Kalli der Peristeri-Spitze.

509. Lecidea petrosa Aln. lilor. 1868. p. 30 exs. An 358 a b.

Var. mula 'l'h. Fr. Scand. P. 511 . - Auf Kälk der Peristeri-Spitzu.

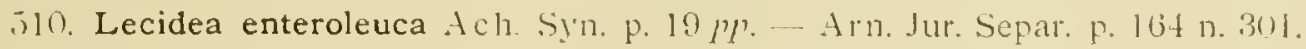

Thallus globuloso-arenlatus aut areolatus, areolae dispersac $l$. congestae. Hypothecium incolor. Pycnosporac arcuatae aut flexuosae $1.5-20$ \%. Ig. 1 \%. It. Thallus KHO non nutatur. - Auf Kalk der Peristeri-Spitze mit Ni. 3.5. - Auf ausgebreiteten sehr kleinen hraunlichen Thalluskörnchen, welche nach den vorbandenen Pycniden zu enlerolenca gehören, sehr zahlreich Tichothicinm ly'smacum Kıb.

511. Catillaria grossa Blonn. Vet. Ak. Wöh. 1767, p. 122. Pers. in Herb. Mong. - Nyl. Prodr. P. 139 sub Licidea. - Syn.: Cat. Mommia kyb. Syst. p. 2:31. - Apothecia ad 1-1.2 mm dian. Discus tandem convexiusculus rugulosus. Hymenium I coerulescit, hic inde tantum impure vinose rubet. Sporac $26-32$ y. 1 g. $15-17 \cdot 5$ !. 1t. (comp. Th. Fr. Scand. p. 581). - Auf Platanusrinde bei Kalentini.

512. Diplotomma alboatrum Arn. Jura. Sep. p. 194. - Hoffm. Enum. p. 30 sub Lichene.

Var. epipolimm Ach. Prod. p. 58 sub Lichene. - Auf dem lialk rom Peristeri ganz unbedoutend entwickelt (leg. Hartl).

513. Rhizocarpon geographicum DC. Fl. franc. 11, p. 365. - 1,in. Spec. plant. 1, p. 1067 sub 1.ich.-. Aul Feldspat der Peristeri-Spitze.

514. Opegrapha trifurcata Hepp. in Nül1. princ. p. 67. - Arn. Jur. Sep. p. 219. - Nur cinige ganz jugendliche Apothecien zwischen Licanora mmorm, welche nach der Form der Asci und Sporen zu Stitzb. Steinb. Opeg. tab. I, fig. $5 k-s$ gehören dürften. - Auf Kalk del Peristeri-Spitze.

515. Arthonia vagans var. Kärbcri Almq. Arth. p. 51. - Hypothecium rufo-fuscum. Epithecium obscure fusco-viride. Sporae $15-20$ \% 1 g. $6-7 \cdot 5$ \%. It. - Auf lialk der Peristesi-Spitze. Ausserden aul den Sreolen der Cal. T'ariabilis, welche dadurch kaum verändert erscheinen. Der übereinstimmende innerc Bau der Apothecien rerbietet eine Trennung dieser syntrophen Form von Arth. Förberi. Allerdings ist hei heiden das Epithecium oft auffallend braungrün und in den Sporen der ersteren, deren Apothecien ölter elliptisch und dann deutlicher convex sind, findet sich nicht selten das tropfenfömige Plasma, welches Nýlander (comp. Hue. Add. p. 25!) für patellulata besonders herrorlnebt. Das dickere. dunklere, zellige Epithecium scheint mir aber für Arth. Körtheri massgebend zu sein.

516. Verrucaria marmorea Scop. Fl. Carniol. p. 367. - Comp. Arn. Verl. A. Z. hot. Ges. Mien 1882. P. 147. - F. lurpurascus Arn. Verh. 1. c. 1872, p.307. - Holfm. Pl. 1.ich. p. 7t. - Auf Kalk ron Jsumerka.

517. Thelidium decipiens Ain. Fl, 1858, p. 55t. - F. limminilioiles Arn. Jur. Separ. p. 260. - Krb. Par. p. 351 Thelid. decip. - IVenig entwickelt auf Kalk der Peristeri-Spitze.

518. Tichothecium pygmaeum krb. Sert. Sud. 10. - Auf den Areolen des Thallus ron Lccidea cuterolenca $\mathrm{Nr} .28$.

519. Cercidospora transmutans n. sp.--Perithecia emergentia tandem subglobosa ad $0.25 m m$ diam.

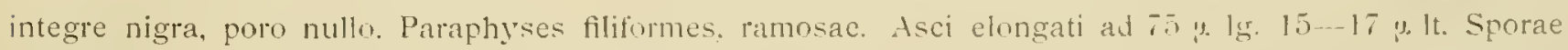
nctonae 22-30 \% lg. $8-11 \%$ 1t. nvato-oblongae. medio salefe leviter constrictae. apicitus rotundatis. I septatae, cellula altera crassiore et saepius etiam evidenter longiore. I contentus tantum asconum lutescit.

Thallus formatur hyphis fuscis, supra ad ji. crassis, thallum alienum, cujus hyphate ct gonidia necantur. percurrentibus. - Auf dem Lager der Lican. mmorm (auf Mergel inn Kalarrytae). Die hefallenen Theile des Wirthes sterben ah und schwärzen sich, nachdem sich das Hyphengewehe des Gastes reicher entwickelt

(Halacsy:! 
hat, Was jedenfalls beim Hervortreten einzelner Perithecien noch nicht der Fall ist. Die Fürbung durch $h H O$ urscheint zuerst nuch lleckwcise, dann bleibt sie ganz aus. Der innere Bau der Perithecien hat vielc Ihnlichkeit mit dem von Arth. fallar. In Betracht kommen litr. alligina Nyl., welche durch die Perithecien und Sporen, und I'ar. imala Nyl., welche durch die Paraphysen und die liom der Sporen verschieden erscheint. Die übrigen mir bekannten Arten von Cicridnspora (vergl. Diblymospllucria Fuckel in Rab. (rypt. Fl., 1. Bd. 11. Abth. P. 432) unterscheiden sich viel bedeutender schon durch die Sporen.

\section{Verzeichniss der Gattungen.}

Die erste Zabl bezieht sich auf dic betreffende Seite des Scparatabdruckes, dic zwcite (in klammern befindliche) auf dic fortlaufende Paginirung des Bandes der Denkschriften der kais. Akademie.

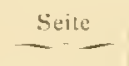

Ahies .......4.43 [250] Acanthus .....34[250]

Acer ..........17 [233]

Achillea ......26 [21"2]

Aclianthnum .....44[260]

Aethionenta .... 13 [229]

Agrimonici . . ..21 [237]

Agroslemma . . . . 14 [230]

Agrostis . . . . . . t2 [258]

Ariar ........t2 [258]

Alichilla ...21 $[237]$

Allium ..... .41[257]

Alopecnrols . . . . 42 [258]

Alsine . . . . . . 16 [232]

Althaea ... . . . . 16 |232]

Alyssum . . . . . 13 [229]

Amarantus ....39 $[255$

Anacamptis . . . t1 [257]

Anagallis

$38[254]$

Andropogon ....42 [258]

Anthemis ......26 [242

Anthyllis...... $18[234]$

Aralis........ 12 [208]

Arintus....... .31 [247]

Avenaria ...... 16 [23:

Armeria . . . . . 38 [254]

Artomisia .....27[243]

Arthonia

$\ldots \ldots+49[265]$

Armun ........ . +1] [257]

Aspermla . . . . . 25 [24]

Asphodeline ....+1 |257

Aspidium

$.4+1260]$

Asplinium .....4t [260

Astragalus .....20|?236]

Astrantic seite

Athamanta

Anbrietia...

Bacidia . . . . . . 49 [265]

Ballota .......35[251]

Barbarca .... . 12 [228]

Bellis........28 [244]

Berteroa . . . . . 13 |229]

Blitum . . . . . 39 |255]

brachypolinm . . 43 [259]

Briza . . . . . . 43|259|

Bromus . . . . . . 42 |258|

Buplcurum ....24|240|

Calamintha ....36|252|

callistemma . . . 26|24:?

Caloplaca .... .47 |263]

ralyeotome .... 17 [233]

Campanmla ....30|246]

Capsella . . . . . 1312029|

Cardamine....12|228

(antuns . . . . .28 |244|

carer ......... 21258|

Carlina.......28|24+7

Carpinus. .....40|256|

Carthanus. . ...28 [244]

Carmm .......2 $2+[240 \mid$

Catillaria .... . 49[265

Centaurea.... . 28 [254]

Centranthus ...26 [242]

C'crastium ......15[2:31]

Cercilosprora ...401265]

cercis ........201236]

Cetract .......t4 |260|

Chamacpente ...28/2447

(henopodium . . . .39) |255)

Chrisonthemum .27|24:3
Gchorinm ....30[246]

Cirsinm ...... $28[2+4$

Cislus ......... $1+[230]$

Clematis ...... 7 [2:23]

Clinopolinm. . . . 30 [2-'2]

Colutea ........ 19 [235]

Concolintus ...32 [2+8]

Comus .......25 [241

Coronilla .... . 19 $\{235$

Corydalis . . . . . 12 [2281

Coloncaster.....21 |237|

(1epis . . . . . . . 29 [245]

Crocts..........+1 |257|

Crupina ......29 [245]

Cuscuta . . . . . 32 [248]

(jnoglossum . . .32|248

cinosurts .....12 12587]

Cypertis........ [258]

Cyslopleris .....t+|260|

Dactylis . . . . . +2 |258|

Daphne . . . . . . 39 |255|

Dancus . . . . . . 23 |239|

Delphinimm ...11[227]

Dianthus. . . . . 15 [231]

Digitalis . . . . . 33 |249|

Diplotomma ... .94 [265]

Doronicum . . . .26 [2+2]

Doricminu . . . . 19[235]

Draba . . . . . . 13/229

Dryis.......

Echium . . . . . 322 [248]

Edrajanthus ....31 [2+7]

Epilobium.....21|237

Epripatis .....+1 [257]

Equisctum.
Erica ......... 31|247

Erigeron .....28|년 $44 \mid$

Erynginm . . . . 24 [240]

Ery'simmm … 13|'230|

Erythraea ....31 [2047

Enplortia ....40 [256]

Euphrasia ...34|250|

Fermlago . . . . 2:3 [239]

Festucar . . . . . . +3 [259]

Filago . . . . . 27 270243

Fragaria...... .21 |237

Fratimus ... . . 31 [?24

Freyera . . . . . 23 $[239$

Fritillaria.....+1 [257]

Galuclites .....28 [2+4]

Galega ...... 19 [235]

Galinm . . . . . 25 |24)

Geraninun ......17|233]

Genm .........2] [237|

Gratolechia.....47|2637

Hil]naldia . . . 43 [359]

Hedera . . . . . . 24 |240|

Helianthemmm . . 14 [230]

Helloborns ....11 |2.27|

Herniarit . . . . 22? |?38|

Hieracium . . . 29 $|245|$

Hiprocrepis . . . 19|235|

Holins. . . . . . . 42 |258|

Hordenin ..... tis [259|

Hyosciamus. . . . 329|248|

Hypricum . . . . 16|232|

Hypolateris ....30 [246]

Iheris.........13|220|

$17+\ldots \ldots \ldots .17|233|$

Juniperus . . . . 135|250| 
$\therefore$

Koclerial .... 12 |258|

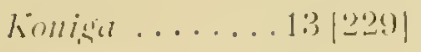

Kinantia . . . . 26 6 |2+2|

Lactuia .......20 20 $|2+5|$

Lamium .......35|251|

latlyyins ....20 [236]

Laturus . . . . . 40 40[256]

Larctira ...... 16 [232]

Licuntia........ $+8[26+]$

Lccomora ....t. $7[2033$

Lecidia .......t9 [0.95]

Lconturton .....30 $\{246\rceil$

Lilinm ......+1 [2557]

Linaria . . . . . . 33 [249] $P$

l.imum . . . . . . 16|232|

Lithosprommm . . .32|248|

Lolinm . . . . . 4. 4:3 [25\%]

Lonictra . . . . . 25 [2-2+1]

Lotus. . . . . . . 19 [235]

Lisimachia ....38 [254]

. Halubaila . . . . 22: [239|

Malcolmia . . . . 1? [2909

Malial . . . . . 16|232|

Marrubium ....36 [252]

. Medicago . . . 18/234|

Wclanlrium . . . 1+ [230]

Melica . . . . . . 429 2258

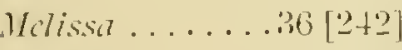

Hentha . . . . . . is [25t]

Micromeriu . . . .36|'-52

Moenclia .... 16 [232]

Hyosotis . . . . . 32 [25:

.Hytus . . . . 21 [237]

Nepeta . . . . . . 36 ['25:2

Nigclla . . . . . . 11 |227|

\section{selle}

Olca . . . . . . . .31

Onobughis . . . .20 [236|

Ononis .......18 [23.1

Onopordon ....28 [244]

Onosma ........32 |248|

Origamum . . . . .3.36 [25:2]

Orlaya ......223 [239]

Orohanche ........ [250]

Orobus .....2.20 [236]

Ostry'.......40 [256]

Palinms . . . . . 17 [233]

Pammaria . . . . 48 [26t]

Papaver .......11[227]

Pedicularis. . . . . 34 [250]

Pencidannm . . . 23|239]

Phlcum . . . . . . t2 $[258$

Phlomis........35 [25]

Plylliria .... .31 [240]

Fhysalis .......32[248] Sarifraga .....23 [239]

Ply'scia ......t6 [262] Scabiosa . . . . 26 [242

Phylolacea .....39[255] Scilla .......44[ [256

Picridium......30 [246] Sclcranthus....222 [238]

Pimpinclla .. . .2+[240] Scolymus ..... $30[246]$

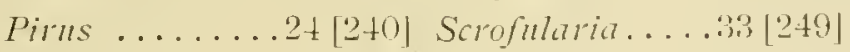

Pisfacia ......17 [233] Scnlcllaria ....535|251]

Plankago......38 [254] Scchm .......22 [238]

Platanus......t0 [256] Sempervitum . . 22 |2038|

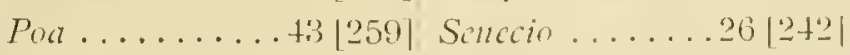

Polanthmm . . . .31 [247| Siskria . . . . . . 22 [258]

Polygala ..... 1+[230] Sherardia. ...25 [2+1]

Polygonum .....39 [255], Sidcritis . . . . . 36 [252]

Polentilla .....21 [237| Silcuc ....... . 1+|230|

Potcrinm .....21 [237] Sisymbrimm ... 13|229]

Primula ......38 [254] Smilax .....41 [257]

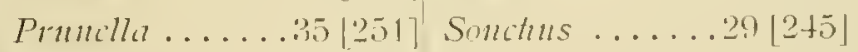

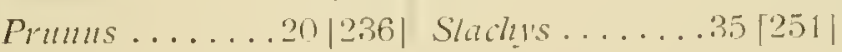

Siene

$2 \overline{8} \mid \overline{411}$

$3|290|$

Stipa . . . . . . 1'2 |258|

Synciloblastus . . 48|26+1

Taraxacam . . . 29) $|2-15|$

Teucrinu .....34|250|

Thelidium ..... . 49|2055|

Thicsinm ... . . 40|256|

Thlaspi . . . . . 1:3 |'209|

Thymuts . . . . . . 36|25.52|

Tichothecinm . . .49|?65|

Torilis .......2? 2334

Tragopogon ....30 [246|

Trifolium ......18 [23.1

Trimia.......2+ $|2-10|$

Trisetum .......+2|2.58|

Tunica .......15|231|

Turseniu . . . . . 23 [239]

Tussilago ....28 $2924 \mid$

Tyrimmus ....228[2+1|

IT/mus . . . . . . 40 $40|256|$

Lrtica .......to $[256]$

Ialantia . . . . . 25 $|241|$

balcrianclla ....26|242|

Veratrmm ...... 1 1 255

Terbascum . . . . . .32!?-18

Icrbona ... . . . 34t |2501

levonica ... . . .33 |0.2.1!1|

limararia .....t?|:65|

Iicia . . . . . . 20 20 |2:36|

1iola . . . . . . 14|230|

likix . . . . . . . 3t |250|

Iritis . . . . . . 17'2333|

Zacjutha .....30|246|

Ziziplenta .......35|251| 


\section{Erklärung der Abbildungen.}

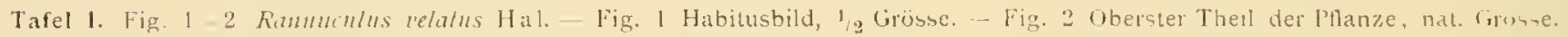
Fig. 3 Cardamine bataraeotides $\mathrm{Hal}$. Etwas rerkleinert.

Tafel II. Fige 1 dehilled chsinthoides Hal. Naturliche Grïsse. rig. 2 Achilled herneri $\mathrm{Hal}$. Natürliche Grösse.

Tafol III. Fir 1 Campanula flagellaris Hal. Natürliche truste. lrig. 2 Thrmus botssieri Hal. Naturliche Groise. 


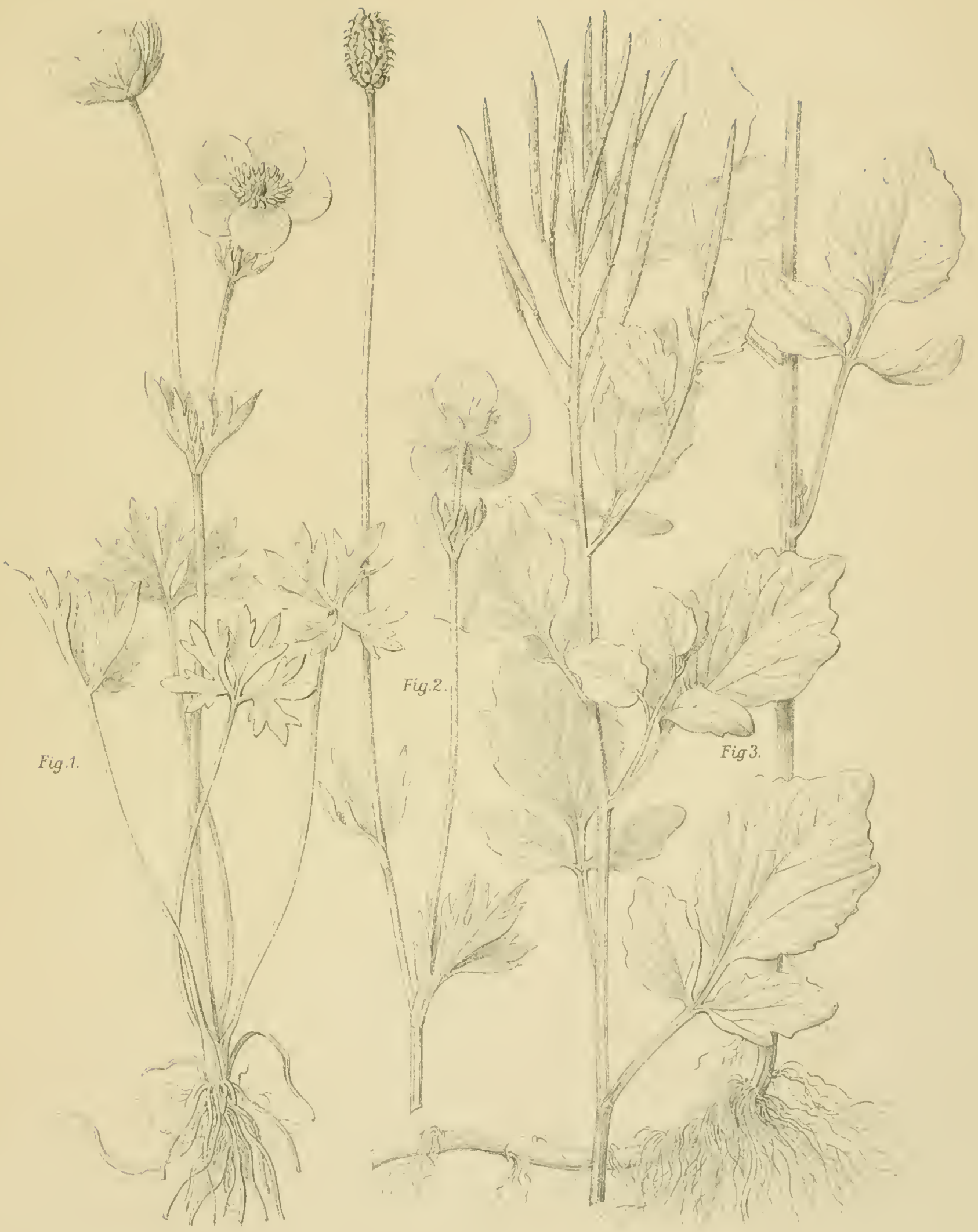

Denkschriften d. kais. Akad. d. Wiss. math.-naturw. Classe, Bd. LXI. 


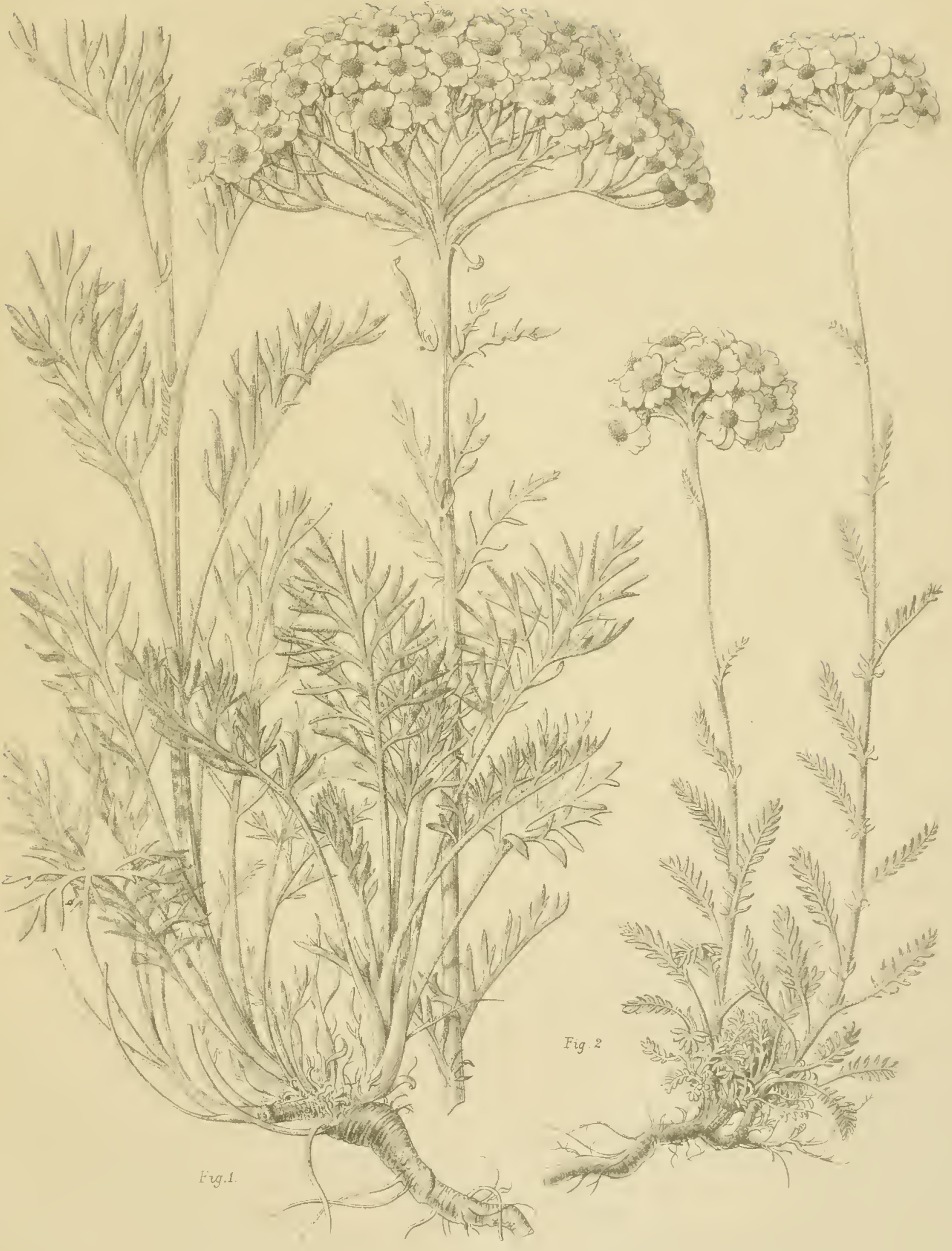

Denkschriften d. kais. Akad. d. Wiss. math.-naturw. Classe, Bd. LXI. 

E.v Halácsy: Flora von Epirus

Taf. III

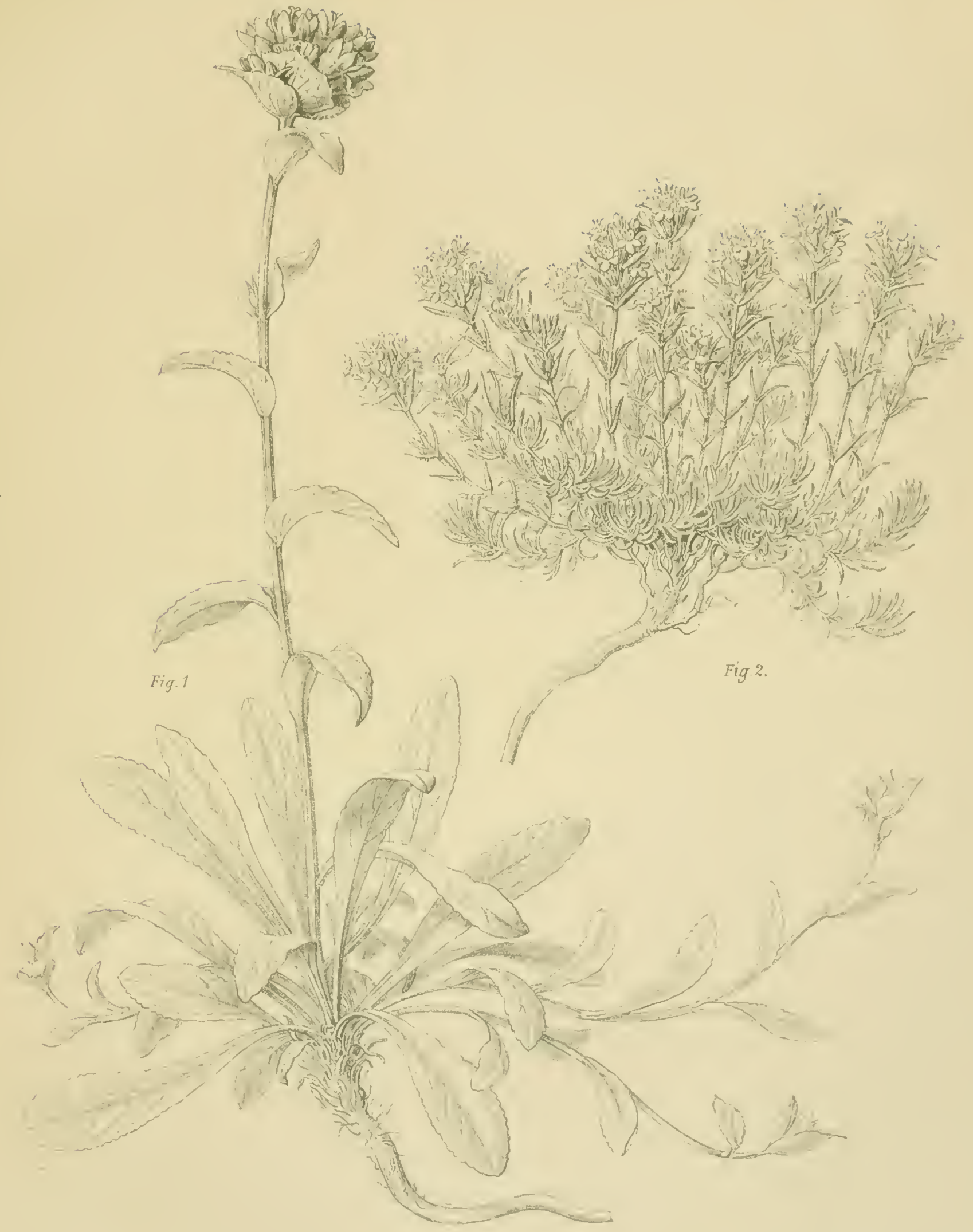

Denkschriften d. kais. Akad. d. Wiss. math.-naturw. Classe, Bd. LXI. 



\title{
BOTANISCHE ERGEBNISSE
}

EINER IM AUPRRAGE DER HOHEN KAISERL, AKADEMIE DER WISSENSCHAFTEN UNTERNOMNENEN FORSCHUNGSREISE

\section{IN GRIECHENLAND.}

\author{
II. BEITRAG
}

ZUR

\section{FLORA VON AETOLIEN UND ACARNANIEN.}

VON

\section{Dr. EUGEN v. HALÁCSY.}

(9Rit 2 Jafeln.)

BESONDERS ABGEDRUCKT AUS DEM LXI. BANDE DER DENKSCHRIFTEN DER MATHEMATISCH-NATURIVISSENSCHAFTLICHEN CLASSE DER KAISERLICHEN AKADEMIE DER IVISSENSCHAFTEN.

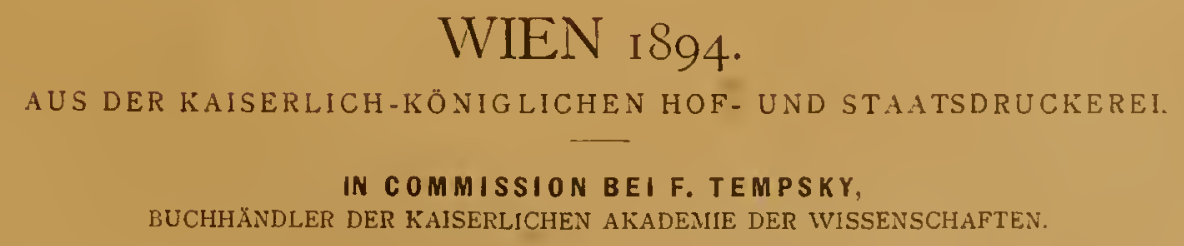

Preis: $\mathrm{I}$ fl. $10 \mathrm{kr} .=2 \mathrm{Mk}$. $20 \mathrm{Pfg}$. 



\section{BOTANISCHE ERGEBNISSE}

EINER IM AUPTRAGE DER HOHEN KAISERL. AKADEMIE DER WISSENSCHAPTEN UNTERNOMMENEN FORSCHUNGSSREISE

IN GRIECHENLAND.

II. BEITRAG

ZUR

\section{FLORA ION AETOLIEN UND ACARNANIEN.}

VON

Dr. EUGEN v. HALÁCSY.

(9kil: 2 Jafelu.)

BESONDERS ABgEDRUCKT AUS DEM LXI. BANDE DER DENKSCHRIFTEN DER MATHEMATISCH-NATURIVISSENSCHAFTLICHEN CLASSE DER KAISERLICHEN AKADEMIE DER WISSENSCHAFTEN.

WIEN I 894.

AUS DER KAISERLICH-KÖNIGLICHEN HOF - UND STAATSDRUCLEREI

IN COMMISSION BEI F. TEMPSKY.

BUCHUÄNDLER DER KAISERLICIEN AKADEMIE DER WISSENSCHAFTEN. 


\title{
BOTANISCIIE ERGEBNISSE
}

EINER IM AUTTRAGE DER HOHEN KAISERL. AKADEMIE DER WISSENSCHAFTEN UNTERNOMMENEN FORSGHUNGSREISE

IN GRIECHENLAND.

II. BEITRAG

ZUR

\section{FLORA VON AETOLIEN UND ACARNANIEN. \\ $\operatorname{VON}$}

\author{
Dr. EUGEN v. HALÁCSY. \\ (OLï 2 STrteln.)
}

VORGELEGT IN DER SITZUNG AN 25. MAI 1894.

\section{Allgemeines.}

Auf meiner keise nach Gricchenland im Sommer 1893 bot sich mir Gelegenheit, die floristischen und pflanzengeographischen Verhältnisse eines Theiles von Aetolien und Acarnanien zu untersuchen. Zunächst hesuchte ich die kleine liüstenebene, welche sich ron Antirrhion bis zum Berge Taphiassos, dem heutigen Kilokovo $(1041 \mathrm{~m}$ ) ausdehnt, und die untere kegion (etwa bis zur Höhe von $100 \mathrm{~m}$ ) dieses mit senkrechten Felswänden in den Crolf von Patras abstürzenden Berges selbst. - Ein zweiter Tag wurde zur Untersuchung der unteren Region des benachbarten Berges Chalkis, des heutigen Varassovo (917 $m$ ) und des an dessen Fusse bei Krioneri liegenden kleinen Sumpfteiches verwendet. - Am 3. Juli setzte ich von P'atras aus über den gleichnamigen Golf und fuln von Kürioneri mit der aetolischen Bahn, über Missolounghi (Mesolongion) und Aetnlikon, nach Agrinion, wo ich noch am selben Tage eine Nachmittagsexcursion in die L'mgebung unternommen habe. - Am 4. Juli fuhr ich von hier über Sorovigli, bei den Seen Rivios und Ambrakia vorüber, nach Kravassaras am Golf ron Arta und botanisirte in der Umgebung dieses Ortes. Den 5. Iuli untersuchte ich die Ostliüste des ambracischen Golfes von Kravassaras über Arapis, Berg Mlakrinorus, Mienidion bis Anina. Denselben Tag erreichte ich noch Arta, den Hauptort des griechischen Epirus.

Dic auf den hier angeführten Strecken vorgelundene Vegetation, ist vollends als eine mediterrane zu bezeichnen.

Die erwähnte, mit mehr minder üppigem Graswuchs bedeckte Ebene bei Antirrhion ist charaliterisirt durch stellenweises, massiges Auftreten von Cymara Cardunculus, Scilla maritima und Asphodilus microcarpus. Eingestreut treten aul: Helianthemum guttatum. Silene vespertina, Trifolium purpurum.

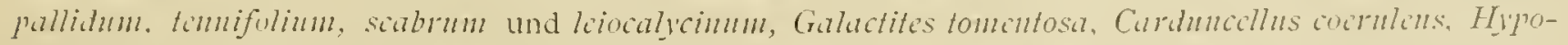
charris cretensis, Seriola acthnensis, Erythraea centaminm und maritima. Plantago Bellardi, Bromus matritusis. Hordium marilimum: aul feuchten mit Schilf bewachsenen Gruben: Serapius lingua, Orchis fragrans, Gludiolus dubius. Elatine actolica. Alopiurns mirulatus. 
Die steinigen . Bhä̈nge der Berge Taphiassos und Chalkis sind mit einem Buschwerke von (Juereus wififa und Poterinm spinosum belieidet, in welehem nebst ciner Anzahl mediterraner kräuter, als woningebende Arten Iboris allica und Slachy's Parolinii erscheinen. Nit dem Zurücktreten dieser Eichengebüsche in den schrofferen Felspartien beginnt dann das Auftreten der aus Plyyllirea modia, Pistucia Lintiscus, Calycolome villosa, Coronilla glauca und Phlomis frulicosa gebildeten Gebüsche, die sich zu schütteren macchicnartigen Bestïnden associiren, aus welchen einzelne Ölbäume hervorragen, und in welchen eine reiche Kräutervegetation sich entfaltet. Von den Arten, die letztere zusammensetzen, verdienen erwähnt zu werden: Paparer Rhocas, Halcolmia zoluchensis. Acthionema gracile, Rescda lymphaca, Sileme nocturna, Tunica glumacea, Linum liburnicum, Malope nalacoides, Malva silvestris, Geranium

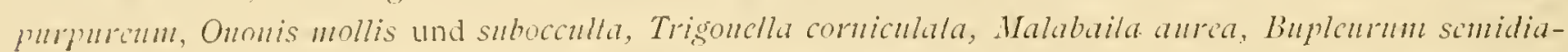
plammm, Orlaya platycarpos, Tordylium apulum, Smymium Orphanidis, Galimm sefaccum, intricalum und atynthimm, Crncianclla latifolia, Shcrardia, Linantia hybrida, Filago spathulata, Tyrimmus, Crepris rulva und Dioscoridis, Picridium picroides. Totpis umbellata, Conrolvulus temissimus, Alkanna orientalis, T'orhascum rigidum. Salvia peloponnesiaca, Balloha acetabulosa, Sideritis purpurea, Planlago

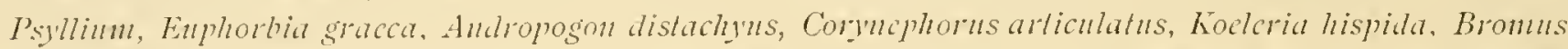
matritcusis etc. In lelsritzen und Felsspalten: Umbilicus horizontalis, Sedmm rubens und eriocarpum, Carum multiflormm, Centranthus Sibthorpii, Phagualon graccum, Chamacpence fruticosa, Contaurca Heldrichii und Cyanms, Campanula drabifolia, Onosma frulescus, Tencrinm Halácsyantum, Micromeria juliana und Eplhadra campylopoda. - Der am Fusse des Chalkis, bei der Bahnstation kirioneri an der Meeresküste befindliche, durch eine unterirdische Quelle gespeiste kleine Süsswasserteich beherbergt in sich und an seinen Ufern eine üppige V'egetation von Sumpfpllanzen. Unter Nassen von Pluragmitcs communis und Cyperns longus wachsen hier: Rumuncmlus opluoglossifolins, Nasturlium officinale, Helosciadium nodiflormu, Sonchus glancscuns, Orchis pulustris, Iris pendacorus, Lemma minor, Juncus muritimus, Scivpus lacustris; an den sumpfigen Ufem: Althaca officinalis, Lyllwum Gracfferi, Sambucus chulus, Galium constrichm, Aspcrula Aparinc, Myosolis lingulala, Lycopus curopacus, Euphorbia pubsicus, Alisma planlago und Polypogon narilimus.

Die Stadt Missolounghi (Mesolongion) liegt in einer nach Süden hin sumpfigen Ebene. Letztere geht nördlich in ein zum Theil mit Mais und Tabak bebautes, zum Theil aber auch uncultivirtes lilachland über, auf welchem vorherrschend Unmengen von Distelgewächsen, wie Scolymms hispanicus. Carthanus lanatus, Onopordon illyriam, Guara Cardunculus, ferner Asphodelus microcanus, Galega officinalis, Allhaca officinalis, Acanthus spinosus, lerbascum simualum. Opopanax hispidus, Erynginm croticum und Sambucus Ebulus gedeihen. Stellenweise finden sich weite Strecken mit dem Adlerfarne überzogen, oder mit kleineren oder grösseren Beständen von Paliurus hewachsen. Einzelne Ölbüume vervollständigen das Biłd dieser an und für sich eigenartigen Vegetation.

Ahnliche Vegetationsverhältnisse finden sich weiter nördlich an der Bahnstrecke bei Aetolikon bis Agrinion, ja mit einigen Abänderungen bis Kravassaras. Duch treten hier schon stellenweise aus Oucrus Acgilops und pubiscens bestehende Wälder auf, welchen Eichen sich cinzelne Pirms amugdaliformis und Callis anstralis beimischen, und deren Unterholz aus Paliurus, Corcis und Qucrus cocifera gebildet wird. Cirsium itulizum überzieht oft grosse Strecken des unbebauten Flachlandes. An den Seen Rivios und Abrakia, welche durch einen kurzen, schmalen, überbrückten Canal verbunden sind, wuchert in dichten Colonien I'itex asums castus.

Die Hügel um Kravassaras selbst werden durch eine Phlomis-Flur (Ph. fruticosa) beherrscht, aus welcher einzelnstehende Öbäume hervoragen und die auf ihrem Grunde eine magere, im Sommer zum grössten Theil verdorte Mediterranflora beherbergt. Von interessanten Arten in iln wären nur Malcolmia viluchensis und Scaliosa lenuis herrorzuheben.

Die Ostküste des ambracischen Golfes, von kravassaras bis Nenidion im Norden, ist dicht mit den immerwüun Natcehien bewachsen, welche schon in den Beitrïgen zur Flora des Epirus geschildert wurlen, auf welche Publication hiemit hingewiesen werden soll. 


\section{Aufzählung der gesammelten Arten.}

Es werden hier nu diejenigen Arten aufgezählt, welche in Gicchenland nicht allgemein verhreitet sind und von diesen auch nur jene, die aus den genannten Provinzen noch nicht, oder bei einigen selteneren Arten von anderen Standorten, bekannt sind. Da Actolien und Acarnanien jedoch nur ven wenigen Floristen bisher besucht wurden (Spruner, Samaritani, Guicciardi, Nieder, Heldreich), so ist dic verhältnissmässig anschnliche Anzahl der aufgezïhlten Arten cine erkläiliche.

1. Ranunculus ophioglossifolius Vill. Hist. pl. Dauph. 111, p. 731 (1789).

Aetolien. Im Sumpfe bei Krioneri am Fusse des Chalkis.

2. Ranunculus trichophyllus Chaix in Vill. Hist. pl. Dauph. 1, p.335 (1786).

Actolien. In Wassergräben bei Antirrhion.

3. Malcolmia bicolor Boiss. et Hleldr. Diagn. Pl. or. Scr. I, Nr. VI, p. 10 (1845).

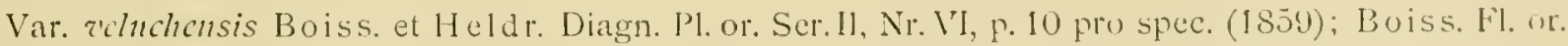
I, p. $229(1867)$.

Aetolien. Am Fusse des 'Taphiassos.

Acarnanien. Auf Hügeln bei Kravassaras.

Boissier unterscheidet diese Art von den übrigen mit nicht stengelumfassenden Blättern versehenen Arten der Section Eunalcolmia vor Altem durch die nicht ausgerandeten Petalen. Dieses Merkinal ist jedoch nicht durchgreifend, da oft an einem und demselben Individum ausgerandete und nicht ausgerandete Petalen vorkommen.

Durch dic kleinen rosenrothen, am Nagel gelben Blüthen und die verhältnissmässig kurzen, mit cincm dicken, kurzen Stiel verschenen Schoten, ist sie ron der ebenfalls kleinblüthigen IV. chia (Lam.) leicht zu unterscheiden. M. gracca Boiss. et Sprun. und M. maritima (L.), die übrigens, wie Haussknecht schon in Symb. ad flor. grace. p. 13 (1S93) hervorgehoben hat, von einander nicht specifisch verschicden sind, weichen schon durch die tiefvioletten viel grösseren Petalen von dicser Art ab.

4. Alyssum orientale Ard. Spec. alt. p. 32 (1763).

Aetolien. Auf Kalkfelsen des Chalkis und Taphiassos.

5. Iberis attica Jord. Observ. sur plus. pl. nouv. VI, p. 42 (ISti). I. Jordani Boiss. Diagn. Pl. or. Ser. II, Nr. 1, p. 38 (1853).

Actolien. In Büschen von Quercus coccifera am Fusse des Taphiassos häufig.

Boissier glaubte den von Jordan gegebenen Namen, wegen des Nichtvorkommens der Art in Attica, nicht acceptiren zu können und benannte dicselbe a. a. O. neu als I. Jorlani. Nach Mittheilungen Heldreich's, der sie übrigens schon vor Jahren auch am obenangeführten Standorte gesammelt hatte, liommt sie zweifellos auf dem Hymettus vor, wic auch auf den Inseh Corfu und Santa Maura. Der Jordan'sche Name hat daher, abgesehen von der Priorität, auch aus letzterem Grunde seine rolle Berechtigung.

0. Aethionema gracile DC. Syst. 11, p. 559 (1821).

Aetolien. Auf dem Taphiassos und Chalkis.

7. Reseda tymphaea Hausskn. in Mittheil. Thür. bot. Ver. V, p. 10 (188i) et 1893, p. 41. Var. asperula.

Differt a typo caulibus inferne papilloso-asperulis.

Aetolien. Im Felsenschutte am Fusse des Chalkis. Von Heldreich auch auf der Kakiskala des benachbarten Taphiassos gefunden.

Von der zunächst verwandten $R$. inodora Reichenb. hauptsächlich durch die viel kürzeren Blüthensticle und die kurzen, breit dreieckigen Kapselzähne verschieden. 
8. Helianthemum guttatum L. Sp. pl. p. 526 sub Cisto (1753). - Tuberaria tariubilis, \% rulgaris, \% Linnaci llillk. et Lange Prodr. 11. Hisp. 111, p. 721 (1880).

Actolien. Grasplätze bei Antirrhion.

9. Silene rigidula Sibth. et Sm. Fl. Graec. Prodr. 1, p. 299 (1800).

Acamanien. Sonnige Hügrel bei Kravassarats:

10. Silene nocturna L. Sp. pl. p. 416 (1753).

Actolien. Am Fusse des 'Taphiassos und Chalkis.

11. Silene vespertina Retz. Obs. but. I11, p.31 (1783). - S. hispida Desf. Fil. att. I, p. 348 (17as). Aetolien. Wiesen bei Antirrhion.

12. Silene trinervia Seb. et Maur. Fl. Roman. p. 152 (1818).

Acarnanien. 1 Gebüschen an den Ufern des Acheloos bei Sorovigli und hei Kravassaras.

13. Tunica fasciculata Marg. et Reut. Essai d'une flore de l'ile de Zante, p. 31, t. 1 sub Gypsophila (1838); Boiss. Fl. or. suppl. p. 82 (1888). - Gypsophila fasciculala Boiss. Fl. or. I, p. 55á) pro parte.

Acarnanien. Steinige, kahle Hügel bei Agrinion.

14. Tunica glumacea Bory et Chaub. in Exped. scient. Nor. 1Il, 2, p.340 sub Diantho (t 832); Boiss. F1. or. I, p. 517 (1867).

Aetolien. Am Fusse des Chalkis.

15. Dianthus viscidus Bory et Chaub. in Exped. scient. Mor. HI, 2, p. 119 (1832); Boiss. Fl. or I, P. 509 (1 807$)$.

Acarnanien. Bei Kravassaras und Arapis.

10. Elatine aetolica Hal. et W' cttst. Sp. nova.

Plantula erecta glabra, debilis, ad $10 \mathrm{~cm}$ alta; caule basi radicibus adventiciis tenuissimis rerticillatim dispositis obsito, in parte superiori pseudo-dichotome diviso; foliis parris oppositis, spathulato-linearibus, in petiolum bevissimum attennatis; floribus tetramcris, axillaribus vel in parte superiori caulis in cymam dispositis et tunc bracteolis binis suffultis, omnibus pedunculatis, pedunculo flore longiori vel eum aequante; calycis laciniis obtusis; petalis ovato-lanceolatis acuminatis, calycis lacinias duplo superantibus; staminibus 4, petalis brevioribus; ovario subgloboso, 4 loculare, loculis multiovulatis; stylis 4, brevibus; capsulis calyce et corolla persistente coronatis, carpellis papyraceis, mox deniscentibus, calycis laciniss multo longioribus, inllatis, initio violaceis; placentis columna centrali alfixis, mox evancscentibus; seminibus minimis, fuscis, cylindricis, rectis, subcostatis.

Aetolien. In ausgetrockneten Lachen der Ebene bei Antirrhion, am gegen den Taphiassos zu führenden Wege.

Dic eben beschriebene neue Art weicht von ailen anderen bisher bekannten Arten wesentlich ab, fügt sich abcr in sehr natürlicher Weise zwischen dieselhen ein. Man kannte bislang Arten mit vollkommen dimeren und trimeren Blüthen, ferner Arten mit trimerer Blüthenhülle und hexameren Androceum, endlich Arten mit tetramerer Blüthenhülle und oetomeren Androeceum. Arten mit vollständig tetrameren Blüthen, (tlso der Fumel $\mathrm{C}_{4} \mathrm{~K}_{4} \mathrm{~A}_{4} \mathrm{G}_{4}$ entsprechend, waren hisher nicht bekannt, ihre gegenwärtige oder cinstige Existenz musste aber angenmmmen werden, um den verschiedenen Blüthenbau der beliannten Arten zu erkiären. Die Auffinutung einer sulchen Art ist daher von grossen Interesse, sie spricht zugleich auch für dic Zusammengehörigkeit der hisher hekannten Arten in eine Gattung, mithin für die Unhaltbarkeit der unterschiedenen Gattungen Crypta Nutt. und Birolia Bell.

Die neue Art gehört gemïss der Mlonographic der (iattung (Seubert, Elatinarum Monographia in nova acta Leop. Carol. Acad. XXI, 1, 18tī) in die Section Crypta ("stamina petalis numero aequalia«), stelzt aber zweifellos der in eine andere Section gehörigen E. macropoda Guss. Fl. Sic. p. 47í am nächsten. Damit ist zugleich auch die Unnatürlichkicit dicser Sections-Eintheilung gegeben.

Alit E. macropoda Guss. stimmt sie in den langen Blüthenstielen und der tetrameren Bläthenhülle überein; sie unterscheidet sich aber ron ihr deutlich durely das tetramere Androeceum, die geraden Simen 
und die langen spitzen Petalen. Die Verwandtschaft der beiden Arten wird durch einen Umstand bewiesen, der überhaupt für die Systematik der Elatineen ron Interesse ist. Dic typische E. macropoda hat acht Statubgelïsse in jeder Blüthe, vier epipetale und vier episepale. Seubert beschreibt a. a. O. eine Varietät erecta $=$ E. Fabri Gren., bei der die vier epipetalen Stamina zwar vorhanden sind, aber bedeutend kleiner erscheinen. Diese Varjetät deutet einen Übergang zu der neuen Art an, bei der die epipetalen Stamina gänzlich fehlen.

Die voranstehende Diagnose wurde nach Exemplaren angefertigt, die der forma terrestris angehören. Es dürfte wohl auch bei dieser Art ausserdem eine forma amplibia und eine forma aquatica geben.

lch führe Wettstein als Conautor derselben an, da er die neue Art ebenfalls untersuchte, und mir werthvolle Daten bezüglich der Verwandtschaftsverhältnisse derselben mitzutheilen die Freundlichkcit hatte.

17. Linum liburnicum Scop. Fl. Carn. ed. 2, I, p. 230 (1772). - L. corymbulosum Reichenb. lil. germ. exc. p. 834 (I832). Conf. Parl. Fl. Ital. V, p. 277.

Aetolien. Bei hírioneri am Fusse des Chalkis.

18. Linum spicatum Pers. Syn. I, p. 336 pro var. L. stricli L. (1805). - L. séSsiliflornm \% Lam. Enc bot. 111, p. 523 ( 1789$)$.

Acarnanien. Bei Agrinion auf sonnigen Hügeln.

19. Malope malacoides L. Sp. pl. p. 692 (1753).

Aetolien. Am Fusse des Chalkis und bei Missolounghi.

Acarnanien. Bei Kravassaras.

20. Hypericum Spruneri Boiss. Diagn. Pl. or. Ser. I, Nr. S, p. 112 (1849).

Acarnanien. Auf trockenen Hügeln zwischen Quercus coccifera bei Agrinion.

21. Geranium purpureum Vill. Hist. pl. Dauph. III, p. 374 (1789).

Aetolien. Im Felsenschutte am Fusse des Chalkis.

22. Ononis reclinata L. Sp. pl. p. 1011 (1763).

Var. mollis Savi in Mem. soc. ital. IX, p. 351 pro specie (1802) - O. Chorlcri Desf. Fl. Atl. 11. p. 148 (1800) an L.? - O. reclinala var. minor Mloris FI. Sard. I, p. 421 (1837).

Aetolien. Auf dem Taphiassos.

23. Ononis subocculta Vill. Prosp. hist. pl. Dauph. p. 11 (1779). - O. Columunae All. Fl. Ped. I, p. 318 (1785).

Aetolien. Auf dem Taphiassos.

24. Trigonella corniculata L. Sp. pl. ed. 2, p. 1094 (1763).

Aetolien. Auf dem Taphiassos.

25. Trifolium purpureum Lois. Fl. Gall. 11. p. 125 (1806).

Actolien. In der Ebene bei Antirrhion.

Acarnanien. Bei Kravassaras und Arapis.

26. Trifolium arvense 1. Sp. pl. p. 769 (1753).

Acarnanien. In Gebüschen bei Sorovigli.

27. Trifolium pallidum W. et K. Pl. rar. Hung. I, p. 35 (1802).

Aetolien. Bei Krioneri am Fusse des Chalkis.

28. Trifolium tenuifolium Ten. Fl. Nap. Prodr. p. XLIV (18/1).

Aetolien. Grasplätze bei Antirrhion.

29. Trifolium leiocalycinum Boiss. et Sprun. Diagn. Pl. or. Ser. I, Nr. 2, p. 31 (1843).

Aetolien. Bei Antirrhion.

Acarnanien. Bei Arapis, sehr häufig.

(v. Halàcsy.) 
30. Coronilla glauca L. Sp. pl. cl. 2. p. 1047 (1763).

Aetulien. Auf Kalkielsen am lusse les Chalkis.

31. Galega officinalis 1.. Sp.pl. p. $71+(1753)$.

Actolien. In Gebüschen bei Missolounghi.

32. Umbilicus horizontalis Guss. F1. Sic. Syn. 1, p. 513 sub Cutyledone (18+2); DC. 1'rudr. 111, p. 400 $(1828)$

Aetolien. Kalkfelsen am Fusse des Chalkis.

33. Sedum eriocarpum Sibth. et Sm. Fl. Graec. Prodr. I, P. 310 (1806).

Aetolien. Im Felsenschutte am Fusse des Chalkis.

34. Sedum rubens L. Sp. pl. p. 432 (1753).

Aetolien. Nit voriger Art.

35. Opopanax hispidus Friv. in Flora I835, p. 333 sub Ferula; Griseb. Spic. Fl. Rum. et Bithyn. I, p. 378 (1843). - O. oriculale Boiss. in Ann. scienc. nat. 184t, p. 330.

Aetolien. Bei Missolounghi.

36. Malabaila aurea Sibth. et Sin. Fl. Graec. Prodr. I, p. 192 sub Heracleo (1806); Boiss. Fl. or. II, p. $1053(1872)$.

Aetolien. kalkgerölle auf dem Taphiassos.

37. Carum multiforum Sibth. et Sm. Fl. Graec. Prodr. I, p. IS8 sub Ithamanta (I800); Boiss. Fl. or. $11, \mathrm{p} .882\left(187^{\circ}\right)$.

Aetolien. An Felswänden am Fusse des Chalkis.

38. Bupleurum semidiaphanum Buiss. Diagn. Pl. or. Ser. 11, Nr. 6, p. 73 (1859).

Aetolien. Auf dem Taphiassos und Chalkis.

Acarnanien. Bei Agrinion und Kravassaras.

39. Smyrnium Orphanidis Boiss. F1. or. 11, P. 925 (1872).

Aetulien. Im Felsenschutte am Fusse des Chalkis.

40. Eryngium creticum 1.am. Dict. IV. p. 754 (1797).

Verbreitet in Aetolien und Acarnanien, von Missolounghi über Agrinium bis hravassaras und Arapis

41. Lagoecia cuminoides 1.. Sp. pl. p. 203 (1753)

Acarnanien. Bei Kravassaras.

12. Sambucus Ebulus L. Sp. pl. p. 269 (1753).

Aetolien. Am Sumpfe bei Krioneri, dann bei Missolounghi.

Acarnanien. Bei Agrinion und Kravassaras.

13. Galium constrictum Chaub. in St. Am. Fl. Agen, p. 67 (1821).

Aetolien. Im Sumpfe bei Krioneri am Fusse des Chalkis.

44. Galium setaceum Lam. Dict. 11, p. 584 (1790).

Aetolien. Auf dem Taphiassos.

45. Galium intricatum Marg. et Reut. Essai d'une llore de l'île de Zante, p. 54 (1S38).

Actolien. Auf dem Taphiassos und Chalkis.

46. Galium zacynthium Marg. et Reut. I. c.

Aetolien. Mit voriger Art auf dem Chalkis.

In der Flora or. 11I, p. 73 wird diese Art olne Notivirung einfach als Symonym zu G. intricatum gehracht. Oflenbar bewog l3uissier hiezu der Umstand, dass heide Arten sich hauptsäthlich nur in der Behaarung unterscheiden. Dieser Unterechied ist jedoch schon in der freien Natur so auffallig. dass man beive Arten schon von der fierne zu erkennen vermag. G. intricutum ist in allen Theilen mehr weniger 
dicht hehart und sieht, wie dies schon dic Autoren ganz treffend hervorgehoben hahen, aschgrau aus: die lirüchte sind hackig-steifhararg, die Blïtter durchschnittlich breiter, kürzer. Dem gegenüber ist G. zacy"mthium fast ganz kahl, nur die Blätter sind oberseits spärlich kurzhaarig: letztere sind zuglcich ctwas glänzend, nicht matt wie die des $G$. intricatum. Ausserdem sind noch die früuchte gänzlich kahl.

Übergänge fand ich keine.

47. Asperula Aparine N. a. Bicb. Fl. Taur. Cauc. I, p. 102 (1808).

Aetolien. Am Sumpfe bei lirioneri.

48. Centranthus Sibthorpii Heldr. et Sart. in Boiss. Diagn. Pl. or. Ser. Il, Nr. 2, p. 119 (1856).

Aetolien. An Felsen am Fusse des Chalkis und des Taphiassos.

49. Scabiosa tenuis Sprun. in Boiss. Diagn. Pl. or. Ser. 1, Nr. 2, p. 11 t (1843).

Acarnanien. Häufig bei Agrinion und Kravassaras.

50. Anthemis tinctoria L. Sp. pl. p. 896 (1753).

Acarnanien. Bei Kravassaras.

51. Filago spathulata Presl. Del. Prag. p. 99 (1822).

Aetolien. Auf dem Taphiassos.

52. Phagnalon graecum Boiss. et Heldr. Diagn. Pl. or. Ser. I, Nr. 11, f. 6 (1849).

Aetolien. Auf dem Taphiassos und Chalkis.

53. Onopordon illyricum L. Sp. pl. p. 827 (1753).

Sehr häufig in Aetolien und Acarnanien, von Missolounghi über Agrinion und Sororigli bis Kravassaras.

54. Cynara Cardunculus L. Sp. pl. p. 827 (1753).

Aetolien. Häufig in der Ebene von Antirthion und Missolounghi.

55. Chamaepeuce fruticosa Desf. Tableau de l'école bot. mus. hist. nat. p. ol sub Carduo (I804): DC. Prodr. VI, p. 658 (1837).

Aetolien. An Felswänden am Fusse des Chalkis. Bisher nur von der Pergfeste Palamedes bei Nauplia in Argolis bekannt. Die Blüthen sind weiss.

56. Cirsium Acarna L. Sp. pl. p. 820 sub Carduo (1753): DC. Fl. Fi: IT, p. 111 (1805).

Acarnanien. Bei Kravassaras.

57. Cirsium italicum Savi Bot. Etrur. 111, p. 140 sub Carduo (1818); DC. Cat. pl. hort. Mlonsp. p. 96 (1813).

Acarnanien. Bei Sorovigli, weite Strecken überziehend.

58. Galactites tomentosa Mocnch. Mcth. p. 558 (1794).

Aetolien. Bei Antirrhion.

59. Tyrimnus lencographus L. Sp. pl. p. 820 sub Carduo (1753); Cass. Dict. scienc. nat. LVI, p. 33. (1826).

Aetolien. Auf dem Taphiassos.

Acarnanien. Bei Sorovigli.

60. Carduncellus coeruleus L. Sp. pl. p. 836 sub Carthamo (1753); DC. Prodt. 17, p. 615 (1837).

Aetolien. Wegränder bei Antirhion, selten.

61. Centaurea Heldreichii Spccies nova.

E sectione Phalolepis Boiss. Fl. or. 11I, p. 615.

Rhizonate pluricipiti, caespitoso, collo dense albo-tomentoso; caulibus crectis rel adscendentibus, angulatis, paniculato-ramosis, foliosis, incano-tomentosis; foliis rosularum sterilium et caulinis inferioribus longepetiolatis, bipinnatisectis albotomentosis, laciniis oblongolinearibus acutis mucronulatis: foliis mediis et superioribus abbreviatis pinnatisectis canotomentosis; capitulis majusculis, glnhosis. follis summis indivisis vel trilobis bracteatis; involucri glaberrimi phyllis striatis, in appendices scariosas hyalinas lateorhi- 
culatas laceras centro pallide fuscas breviter molliterque aristatas abeuntibus; flosculis purpureis radiantibus; acheniis puberulis, pappi albi serie intermedia achenio sesquilongiore, interiore eo dimidio breviore.

Stengel $20-30 \mathrm{~cm}$ hoch; grundständige Blätter sammt Stiel 10-20 $\mathrm{cm}$ lang; Köpfchen $16 \mathrm{~mm} \mathrm{im}$ Durchmesser.

Aetolien. An den senkrechten Felswänden đes Chalkis bei Krioneri, in Gesellschaft ron Chamaepence fruticosa und Tencrimm Halicsyanmm.

Eine prächtige, in den Blättern und der Bekleidung, wie auch im Habitus an C. cinerca Lam. oder C. Nicderi Heldr. erinnernde Art. Durch den Bau der Hüllschuppen von diesen jedoch wesentlich abweichend und wie schon ohen hervorgehoben gar nicht in die Section Acroloplus, sondern in die Section Phalolcpis gehörig, in welcher Section übrigens C. Heldreichii mit keiner der beschriebenen Arten confundirt werden kann.

Ich benenne die neue Art nach meinem lieben Freunde Th. v. Heldreich, dem rastlosen Erforscher der Flora Griechenlands, der seit 25 Jahren mit bestem Wissen und können, meine derselben Flora gewidmeten Bestrebungen nach jeder Richtung hin unterstützt.

62. Centaurea Cyanus L. Sp. pl. p. 911 (1753).

Aetolien. An Felsen des Chalkis.

63. Centaurea Guicciardii Boiss. Fl. or. HI, p. 661 (1875).

Acarnanien. Steinige Hügel bei Agrinion.

64. Sonchus glaucescens Jord. Obs. V', p. $75(18+7)$.

Actolien. Im Sumpfe von Krioneri.

Acarnanien. Bei Kravassaras.

65. Crepis rubra L. Sp. pl. p. 806 (1753).

Aetolien. Auf dem Taphiassos.

66. Hypochaeris cretensis L. Sp. pl. ed.2, p. 1139 sub Scriola (1763); Bory ct Cha ub. in Exp. scient. Mor. Ili, 2, p. 237 (1832).

Aetolien. Bei Antirrhion.

67. Tolpis umbellata Bert. rar. Lig. pl. dec. I, p. 13 (1803).

Aetolien. Auf dem Taphiassos.

68. Xanthium spinosum L. Sp. pl. p. 987 (1753).

Acarnanien. Wüste Plätze in Agrinion.

69. Campanula drabifolia Sibth. et Sm. Fl. Graec. Prodr. I, p. 142 (1806).

Aetolien. Am Fusse des Chalkis.

70. Erythraea Centaurium L. Sp. pl. p. 229 sub Gentiana (1753); Pers. Syn. 1, p. 283 (1805).

Aetolien. Bei Antirrhion.

71. Erythraea maritima L. Mant. p. 55 sub Gentiana (1767); Pers. Syn. I, p. 283 (1805).

Actolien. Bei Antirrhion.

73. Cuscuta globularis Bert. Fl. Ital. VII, p. 625 (1847). - C. palaestina Boiss. Diagn. Pl. or. Ser. 1, Nr. 11, p. 86 (1849).

Aetolien. Auf Tencrimm Halácsyanmm auf dem Taphiassos.

73. Heliotropium Bocconi Guss. Ind. hort. Bocc. a. 1825: Fl. Sic. Prodr. I, F. 204 (1827).

Acarnanien. Bei Kravassaras.

74. Echium italicum L. Sp. pl. p. 139 (1753)

Acarnanien. Bei Krravassaras.

75. Onosma frutescens Lam. Jll. J, p. 407 (1791); Enc. meth. IV, p. 581 (1797).

Actolien. An Felsen des Taphiassos und Chalkis. 
76. Alkanna orientalis 1. Sp. pl. p. 133 sub Anchusa (1753); Boiss. 1)iagrn. 11. or. Ser. 1, Nr. 4, p. 40 (1844); Griseb. Spic. F1. Rum. et Bythin. II, P. 58 (1844).

Var. hellenica Boiss. Fl. or. IV, p. 228 (1879).

Aetolien. Am Fusse des Chalkis.

77. Myosotis lingulata Lehm. Asperif. p. 110 (1818). - M. cacspilosa Schultze Fi. Starg. Suppl. P. $11(1819)$.

Aetolien. Am Sumpre bei Krioneri.

78. Verbascum Guicciardii Boiss. et Heldr. Diagn. P1. or. Ser. 11, Nr. 6, p. 127 (1859).

Acarnanien. In den Macchien auf lem Berge Makrinoros bei Menidi am Golf von Arta.

Die Exemplare stimmen sownhl mit der Beschreibung, als auch mit der mir vorliegenden Origina!Phanze Heldreich's vom Fusse des Parnasses überein. Die Antheren der beiden längeren Staubgefässe sind übrigens bei dieser Art nur wenig herablaufend.

79. Verbascum ambracicum. Nova hybrida. - V. Guicciartii Boiss. et Heldr. X I'. simatum L.

Bienne, indumento tenui subdetersili obsitum viridi-canescens; paniculae pyramidatae ramis virgatis erecto-patulis; foliis radicalibus oblongo-spathulatis, breviter petiolatis sinuato-dentatis, caulinis oblongis acutis sessilibus; loribus breviter pedicellatis, dissite glomerulatis; calyce tomentoso ad mediun in lacinias breviter lancenlatas fisso; corolla ampla explanata, filamentis albobarbatis, antheris omnibus reniformibus; capsula rudimentari.

Höhe $1 m$, Kelch $5 m m$ lang, Blumenkrone $3 \mathrm{~cm}$ im Durchmesser.

Acarnanien. In den Macchien aul dem Berge Makrinoros bei Menidi am Golf von Arta unter den Stanmeltern.

Von V. Guicciardii durch die etwas dichtere Bekleidung, die rispige Inflorescenz, die buchtiggezähnten grundständigen Blätter, vor Allem aber durch die durchwegs nierenförmigen, nicht herablaufenden Antheren; - von V. simmatum durch die grossen Corollen und die weisswolligen Staubfäden; - von beiden durch die völlig unentwickelten tauben kapseln verschieden.

S0. Verbascum plicatum Sibth. et Sm. Fl. Graec. Prodr. 1, p. 150 (1806).

Var. rigidum Boiss, et Heldr. Diagn. Pl. or. Ser. 11, Nr. 3, p. 143 pro spec. (18.56); Bois.s. Fl. nl. IV, p. $312(1879)$

Aetolien. Auf dem Taphiassos.

81. Verbascum pulverulentum Vill. Hist. pl. Dauph. II, p. 490 (1787).

Acarnanien. Bei Arapis und Arina.

82. Linaria graeca Bory et Chaub. in Exp. scient. Mor. 111, 2, p. 175 sub Antirhino (1S:32); Chav. Monogr. Antiruh. p. 108 (1833).

Acarnanien. Bei Agrinion.

83. Acanthus spinosus L. Sp. pl. p. 639 (1753).

Aetolien. Bei Missolounghi.

S4. Teucrium Halácsyanum Heldr: in Öst. bot. Zeitschr. XXIX, p. 241 (1879).

Aetolien. Auf Felsen des Chalkis bei Krioneri.

Diese Art kommt hier in der ganz gleichen Form, wie auf dem Taphiassos, wo sie von Heldreich entdeckt und auch von mir gesammelt wurde, vor, wie auch in einer zweiten Form. Letztere bildet mächtige rerschlungene Rasen mit bis $50 \mathrm{~cm}$ langen Stengeln; ihre Blätter sind grün, weniger filzig. lch betrachte diese als Schattenforn?.

S5. Salvia peloponnesiaca Bois., et Heldr. Diagn. Pl. or. Ser. J, Nr. 7 , F. 47 (1846). Aetolien. Am Fusse des Chalkis.

86. Ballota acetabulosa L. Sp. pl. p. 584 sub Mlarubio (1753); Benth. Labiat. p. 59.3 (1832-1836). Aetolien. Am Fusse des Chalkis. 
87. Sideritis purpurea Talbot in Benth. Labiat. P. $7+2(1832-1836)$.

Aetolien. Auf dem Taphiassos.

Acarnanien. Bei Agrinion und Kravassaras.

88. Plantago Bellardi All. Fl. Ped. J, p. 82 (1785). - P. pilosa Pourr. in Act. Tolos. 11I. p. 324 $(1788)$.

Aetolien. Bei Antirrhion.

89. Amarantus deflexus L. Mant. p. 295 (1771).

Acarnanien. Wüste Plätze in Agrinion.

90. Chenopodium ambrosioides L. Sp. pl. p. 219 (17.5.3).

Mit vorigem.

91. Euphorbia graeca Boiss. et Sprun. Diagn. PI. or. Ser. 1, Nr. 5, p. 53 (184t).

Aetolien. Am Fusse des Chalkis.

92. Celtis australis L. Sp. pl. p. 1013 (1753).

Acarnanien. In Vorhölzern bei Sorovigli.

93. Quercus Aegilops L. Sp. pl. p. 996 (1753).

Acamanien. Bei Sorovigli einen ausgedehnten Bestand bildend.

94. Serapias Lingua L. Sp. pl. p. 950 (1753).

Aetolien. Bei Antirrhion.

95. Orchis palustris Jacq. Icon. pl, rar. I, t. 181 (1781).

Aetolien. Im Sumpfe bei Krioneri.

96. Gladiolus dubius Guss. Prodr. Fl. Sicc. Suppl. p. 8 (1832).

Aetolien. Bei Antirrhion.

97. Asparagus acutifolius L. Sp. pl. p. $31+(1753)$.

Aetolien. Am Fusse des Chalkis.

98. Allium margaritaceum Sibth. ct Sm. Fl. Graee. Prodr. I, p. 221 (1806).

Acarnanien. Bei Agrinion.

99. Lemna minor L. Sp. pl. p. 970 (175:3).

Aetolien. Im Sumpfe bei Krioneri.

100. Cyperus longus L. Sp. pl. p. 45 (1753).

Aetolien. Im Sumpfe bei Krioneri.

101. Andropogon distachyus L. Sp. pl. p. 1046 (175.3).

Aetolien. Am Fusse des Chalkis.

102. Alopecurus utriculatus L. Sp. pl. p. So sub Phalaride (1702); Pers. Syn. I, p. 80 (1805).

Aetolien. In Wassergräben bei Antirrhion.

103. Polypogon maritimus Willd, in Verhandl. Ver. Naturfreunde, Berlin 111, p. $4+3$ (1801).

Aetolien. Bei Antirrhion und Kirioneri.

104. Corynephorus articulatus Desf. F1. Atl. 1, p. 70 sub Airil (1798); P. de Beaur. Agrost. p. 90 $(1812)$.

Aetolien. Auf dem T'aphiassus.

105. Koeleria hispida Sari 11. Pis. I, p. 117 sub Festuea (1798); DC. Hort. Monspel. p. 119 (1813). Aetolien. Grasplätze bei Antirrhion.

Neu für das Festland. Yon Bicknell bei Lerkimo auf Corfu im Jahre 1891 gesammelt.

106. Bromus matritensis L. Amoen. acad. IV, p. 265 (1759).

Aetulien. Bei Antirrhion. 
107. Hordeum maritimum With. But. Arrang. 1. 179 (1776).

Actolien. Bci Antirhion.

108. Ephedra eampylopoda C. A. Mey. Monogr. Ephedr. p. $7.3(1846)$.

Actolien. An Felsen am fusse des Chalkis.

\section{Flechten.}

Das Substrat der angeführten Filechten bilden kalkhältiger Sandstein fon Agrinion, Kalke von Antirthion und vom Berge Chalkis.

109. Leeanora (Sect. Aspicilia) concreta Schär. Spic. F. 73.

Var. viridescens Mass. Ric. p. 46.

Acarnanien. Auf Sandstein von Agrinion.

110. Caloplaea (Sect. Pyrenodesmia) intereedens Trer. Lich. Venet. sub Pyrenodes.

F. minuta m.

Thallus insulas minores, tandem confluentes format habitu Cal. intercedentis. Apothecia minuta, vix $0.3 \mathrm{~mm}$ diam. atting. atra nunquam pruinosa, primum immersa deinda adpressa non distincte marginata. Sporae $12-15 \mu \lg .7 \mu$ It. Epithecium $K H O$ impure violascit. Pycnosporae minutissimae ellipticae aut late ellipticae.

Durch die kleinen Apothecien und die Berandung derselben jedenfalis ron frandulenta Krb. verschicden. Theilweise den Thallus von Microth. marmorata durehsetzend auf zwei Stücken Kálk rom Berge Chalkis in Aetolien, so dass kaum anzunehmen, es liege nur eine unentwickelte Form vor.

111. Caloplaca (Sect. Blastenia) arenaria Stnr. - Pers. Ust. Ann. 1794, p. 27.

F. teicholyta Ach. Univ. p. 425. Syn. Calopl. erytlurocarpa Th. Fr. Scand. p. 181.

Die normale Pflanze mit gelapptem Thallusrand, aber spärlich entwickelt. Auf einem Randlappen befinden sich Pycniden mit fast einfachen bis $25 \%$ langen Basidien, kolbenförmigen Paraphysen und kurz stabförmigen 4-7 $\mu_{\text {. }}$ g., $1-1 \cdot 5 \mu_{\text {. }}$ t. Pycnosporen. Unsere Kenntniss ïber die Variabilität der Nebenfruchtformen der Flechten ist bislang nicht gross. Aber hier kann doch mit ciniger Sicherheit gesagt werden, dass die beschriebenen Pycniden zu Cal. archaria nicht gehören dürften. Eher stimmen sie zu Lec. conareta f. viridescens, welche fast unmittelbar neben C. arencuria wächst. Ist diese Ansicht richtig, dann zeigt der Fall, dass Flechten in fremden Wirten auch nur mit Nebenfuchtformen in die Erscheinung treten können. Eine nähere Untersuchung war nicht ausführbar, da das Object geschont werden musste.

Acarnanien. Auf Sandstein von Agrinion.

112. Buellia (Sect. Diplotomma) alboatra Th. Fr. Scand. p. 607. - Hoffm. Enum. p. 30 sub Lich.

Var. margarilacea Smrf. Lapp. p. 148. - Apothecia mox convexa, leviter pruinusa.

Acarnanien. Auf Sandstein von Agrinion.

\section{Diploschistes gypsaceus.}

Var. coloralus Stnr.

Habitus, excepto colore albissimo, et sporae minores et minus septatae speciei. Thallus J non coloratur, $\mathrm{CaCl}_{2} \mathrm{O}_{2}$ roseo rubet, $\mathrm{LHO}$ primum lutescit, deinde obscure purpurascit. Substantia colurata serius in $\mathrm{KHO}$ colore cocruleo viridi solvitur. Pycnides non vidi.

Die Sporen schwanken zwischen 20-27 $\mu$ lg., 10-12 j. 1t., sehr selten werden sie bis $15 \mu$ breit und bleiben öfter 3 sept. 1 div.

Acarnanicn. Auf Sandstein von Agrinion.

1 Bearbeitet von J. Steiner. 
114. Placidium tapeziforme Mass. Sched. p. 114.

Acarnanien. Wenig entwickelt auf Sandstein ron Agrinion.

115. Verrucaria (Sect. Lithoicea) viridula Ach. Univ. p. 675. - Schrad. Spic. 192 sub Endoc. Sporte $23-30 \mu \mathrm{lg}, 12-16 \mu$ lt. mox leviter lutescentes.

Actolien. Auf kalk von Antirrhion.

116. Verrucaria marmorea Scop. Fl. carn. p. 367. - Comp. Arn. Verh. d. zool. bot. Ges. Wien 1882, p. 147 .

F. purpuraescens Arn. Verh. 1. c. 1872, p. 307. - Hoffm. Pl. Lich. p. 74.

Aetolien. Auf Kalk des Chalkis.

117. Microthelia marmorata Krb. Par. p. 398. - Hepp. in litt. ad Arn. sub Phacosp.

Sporae $25-30 \mu \mathrm{lg} ., 12-16 \mu \mathrm{lt}$, raro cellula tenuiore iterum scptata.

Aetolien. Auf zwei Stücken Kalk vom Chalkis.

118. Cercidospora epipolytropa Ainld. Mudd. Nan. p. 298 sub Thalid.

In nicht bestimmbaren sehr kleinen, weissgrauen Areolen, welche Pycniden zeigen mit einfachen Sterigmen und kurz stabfömigen Pycnosporen $3 \cdot 7-5 \mu$.g., $1 \cdot 5 \mu 1 \mathrm{t}$. Die Sporen der Curciclospora sind $18-25 \mu \lg , 5-6 \mu$ t. und erscheinen hie und da undeutlich 3 sept., so dass es fraglich bleibt, ob nicht eine zu trennende Art vorliegt.

Acarnanien. Auf Sandstein von Agrinion. 


\section{Verzeichniss der Gattungen.}

Dic erste Zahl bezicht sich auf die betreffende Scitc des Separatabdruckes, die zweite (in Kilammern befindliche) auf dic fortlaufende P'aginirung des Bandes der Denkschriften der kais. Akademie.

\begin{tabular}{|c|c|c|c|}
\hline & & & \\
\hline$\ldots 91$ & chopodium ...1 & ordcum .....11 [319] & $n \ldots$ \\
\hline thionema & $v \sin m$ & Iypericum .... . 5 [313] & 10 \\
\hline thanna & monilla & Hypochaeris . . . $8[316]$ & \\
\hline$l i m m$ & $4 s \ldots 10$ & & \\
\hline 3] & $\ldots \delta$ & & \\
\hline 1] & .. $s[$ & & - hi \\
\hline .10 &. $.7[:$ & $\operatorname{cia} \ldots \ldots$. & (1). \\
\hline 8]: & iperus........10 [ & $a \cdot \ldots 1$ & 7 \\
\hline 5] & & 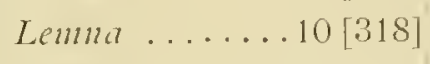 & $\cdots$ \\
\hline 8) & & Lim & 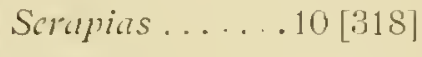 \\
\hline 7 & & Linum . . & is \\
\hline al & - & .66 & \\
\hline 8] & 4 & . tcotarts & \\
\hline 9$]$ & c 1211 & . & $S$ \\
\hline 47 & $67314]$ & Mi & $m m$ \\
\hline .1 & 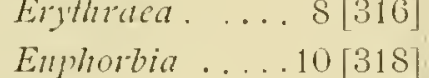 & $M$ & Tolp \\
\hline t] & & Ono & Trif \\
\hline 5] & & Ono & $\tilde{j}[$ \\
\hline 4] & 7[ & Onosma ... & $T u$ \\
\hline 1 & $6[:$ & Opopanax. & 8 \\
\hline 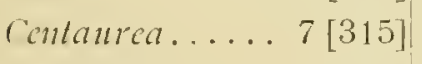 & 5[ & $\ldots 10$ & $L^{\prime} n$ \\
\hline$\cdots$ & $10[318]$ & & \\
\hline 10 & & & Verrucaric \\
\hline 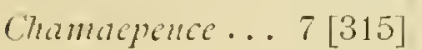 & & go . . & SI \\
\hline
\end{tabular}




\section{Erklärung der Abbildungen.}

Tafel I. Fig. 1-4 Elatine aetolica Hal. et Wettst. - Fig. 1 Btüthe 35/1. - Fig. 2 aufgesprungene Fiueht 30/1. - Fig. 3 Samen, trucken; Fig. 4 gẹuollen 30/1. - Fig. 5-8 Teucrimu Haldicsumum Heldr. - Fig. 5 Habitusbild, nat. Gr. - Fig. 6-7 kielch, vergr. - Fig. 8 Btumenkrone. vergr.

Tafel II. Centurral Heldrichii Hal. - Fig. 1 Habitusbild, nat. Gr. - Fig. 2, 3 und 4 untere, mittiere und ohere lü̈llschuppe, vergr. - Fig. 5 Achene, vergr. 


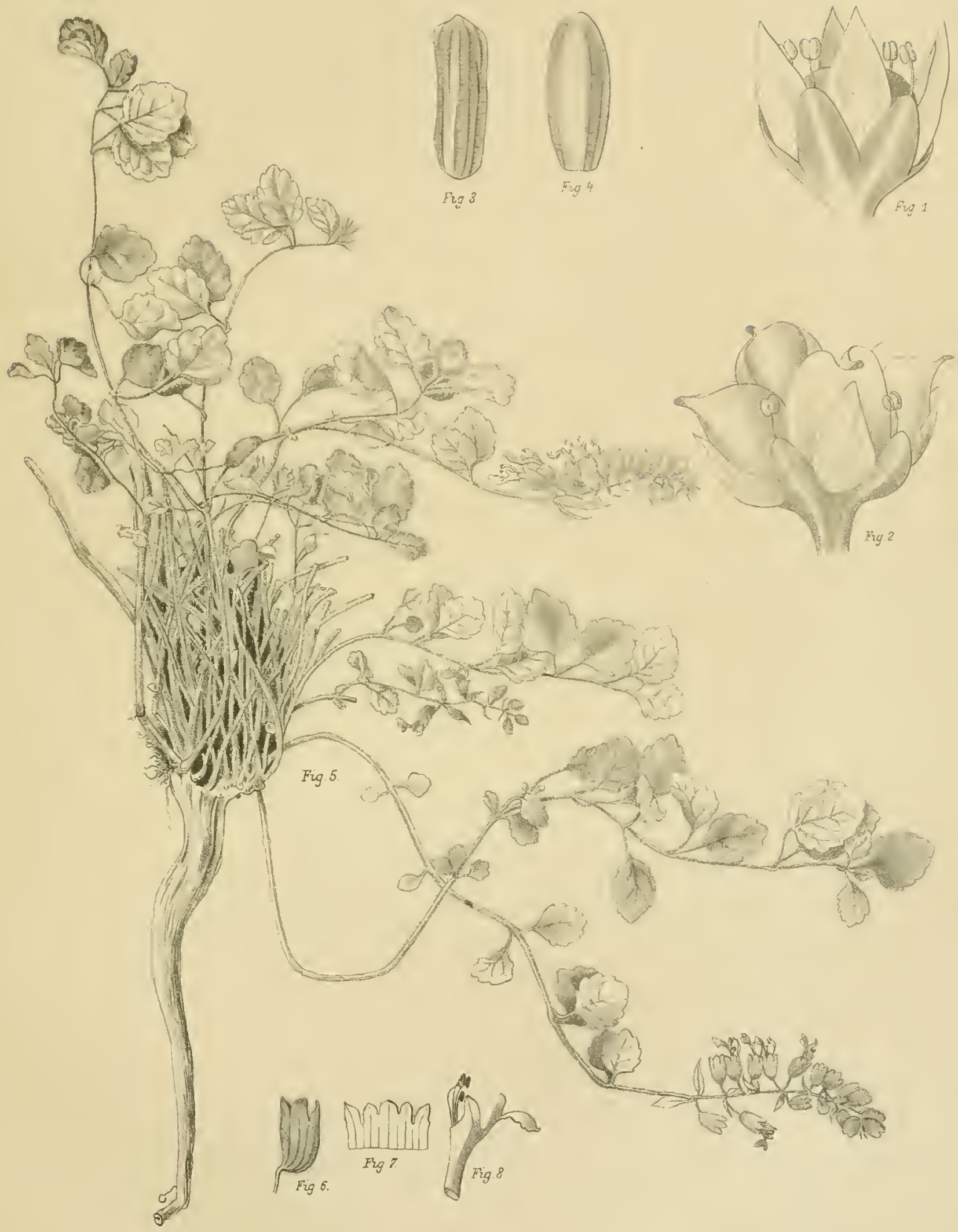



E. v. Halácsy: Beitrag zur Flora von $\Lambda$ etolien u Acarnanien.

Til: II.

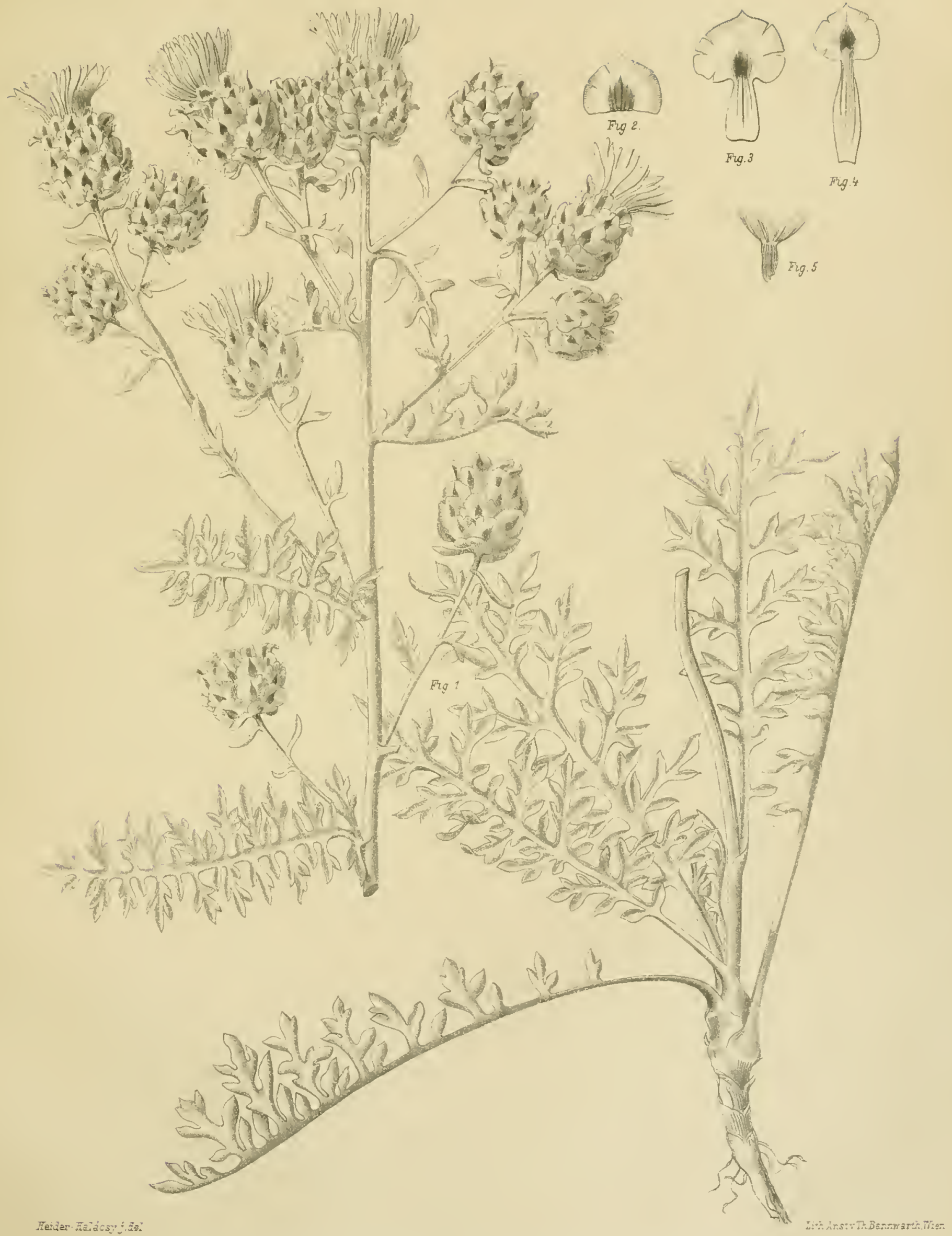

Denkschriften d. kais. Akad. d. Wiss. math.-naturw. Classe, Bd. LXI. 



\title{
BOTANISCHE ERGEBNISSE
}

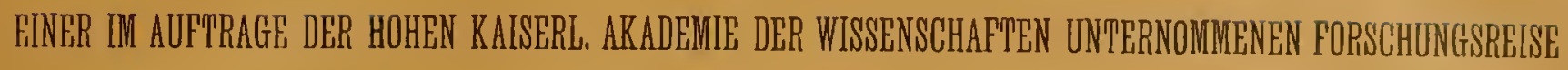

IN GRIECHENLAND.

III. BEITRAG

ZUR

\section{FLORA VON TIIESSALIEN}

VON

\section{Dr. EUGEN v. HALÁCSY.}

\author{
(9kit 2 Jafelu.)
}

\section{WIEN I 894.}

AUS DER KAISERI,ICH-KÖNIGLICHEN HOF-UND STAATSDRUCKEREI.

IN COMMISSION BEI F. TEMPSKY.

BUYHHXNDLFR DER KAISERLICIIEN AKADFMIE DER WISSE,NSCHAFTLA:

Preis: I fl. ro kr. $=2 \mathrm{Mk}$. $20 \mathrm{Pfg}$. 



\title{
BOTANISCHE ERGEBNISSE
}

EINER IM AUFTRAGG DER HOHEN KAISERL. AKADEMIIE DER WISSENSCHAPTEN UNTERNOMMENEN FORSCHUNGSREISE

IN GRIECHENLAND.

\author{
III. BEITRAG
}

ZUR

\section{FLORA VON THESSALIEN}

VON

\section{DR. EUGEN v. HALÁCSY.}

(Qkit: 2 Tafeln.)

BESONDERS ABGEDRUCKT AUS DEM LXI. BANDE DER DENISSCHRIFTEN DER MATHEMATISCII-NATURIVISSENSCHAFTLICHEN CLASSE. DER KAISERLICHEN AKADEMIE DER WISSENSCHAFTEN.

WIEN I894.

AUS DER KAISERLICH-KÖNIGLICHEN HOF - UND STAATSDRUCKEREI.

IN COMMISSION BEI F. TEMPSKY.

BUCHHÄNDLER DFR KAISERLICHEN AKADEMIE DER WISSENSCHAFTEN. 


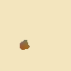




\section{BOTANISCHE ERGEBNISSE}

EINER IM AUFTRAGE DER HOHEN KAISERL. AKADEMIE DER WISSENSCHAFTEN UNTERNOMMENEN FORSCHUNGSREISE

IN GRIECHENLAND.

III. BEITRAG

ZUR

FLORA VON THESSALIEN

ron

Dr. EUGEN v. HALÁCSY.

(פKit 2 Safeln.)

(VORGELEGT IN DER SITZUNG AM 12. JULI 1894.)

1. Allgemeine Übersicht.

Bis in dic jüngste Zeit blieb diese von hohen Gebirgen ringsum begrenzte, in ihrem lnneren zu einer weiten Ebene sich ausbreitende griechische Provinz ein in botanischer Beziehung nul wenig bekanntes Land. Zwar finden sieh schon in Sibthorp und Smith "Florae graecae Prodromus Angaben über das Vorkommen einzelner Arten in Thessalien verzeichnet, welche von Hawkins, einem in Janina ansässigen Arzte, der den Pindus und Pelion Ende des vorigen Jahrhunderts besuchte, herrühren; ${ }^{1}$ dieselben sind jedoch nur' sporadisch und selbstredend nicht im Stande, uns nur annähernd ein Bild der Vegetation des Landes zu geben. Theilweise ermöglichten letzteres schon die Besteigungen des Olymps durch AucherEloy (1836), Heldreich (1851), Orphanides (1857) und Sintenis-Bornmüller (1891).

Finen ganz wesentlichen Fortschritt für die Kenntnisse der Flora Thessaliens brachte jedoch erst Heldreich's zweimonatlicher Aufenthalt im Lande im Jahre 1882, bei welcher Gelegenheit die Umgebung von Volo, das Pelion- und Ossagebirge, das Agya- und Tempe-Thal und die thessalische Ebene bis Larissa durchforscht wurden. Die Resultate dieser Reise hat Heldreich in den Sitzungsberichten der königl. preussischen Akademie der Wissenschaften (1883) veröffentlicht. Im Vereine mit den Ergebnissen der obenerwähnten Durchforschungen des Olymps gestatten dieselben wenigstens über die Vegetationsierhältnisse des östlichen Theiles von Thessalien ein Urtheil zu bilden.

Von einem zweifellos noch grösseren Erfolge gekrönt war Heldreich's in Vereine mit Haussknecht unternommene Bereisung des Landes im Sommer 1885, welche der Erforschung der thessalischen Ebene, insbesondere jecoch der Pinduskette gewidmet war und deren Ergebnisse Haussknecht soeben in den Mittheilungen des thüringischen botanischen Vereines publicirt. Es wurden bei dieser Gelegenheit die

1 Heldreich, Nachträgliches ïber das Vorkommen der Rosskastanie in Sitzungsber. des botan. Ver. der Prox. Branjenburg. XXIV, p. 21. 
Umgebungen von Volo mit dem Pulion, dann die von Velestino, Aivali, Orman-Nlagula, Pharsalos und Karditza, der dolopische Pindus, die Berge Ghavellu und Karava, dann die Umgebung von Trikala und Kalabakia mit den Meteoraklöstern, wie auch jene von Tsungeri und Malakasi, der türkisch-griechische Grenzpass Zygos, die Quellengebiete des Peneios und Achelous, die Ungebungen der Orte Chaliki, Kotura, Vilitza und Frania und der Berg Baba bis zur Ortschaft Klinovo, endlich die Ungebungen der Städte Larissa und Tyrnovo gründlich durchforscht.

Einen nicht unwichtigen Beitrag zur Flora Thessaliens lieferte endlich Forma nek in seinen Beiträgen zur Flora von Serbien, Macedonien und Thessalien. ${ }^{1}$ Formanek besuchte zwar zum Theile die von seinen Vorgängern untersuchten Gebiete, wie das Pelion- und Ossa-Gebirge, mehrere Punkte der thessalischen Ebene, ferner das obere Penciosthal, Malakiasi, Berg Zygos etc. im Pindus, bestieg aber ausserdem noch den Said Pascha und Oxya oberhalb Kastania, die vor ilm noch von keinem Floristen betreten waren.

Mein Besuch Thessaliens selbst dehnte sich leider nur auf wenige Tage aus und galt einigen bereits berührten Punkten. Am 18. Juli kam ich vom Peristeri, über đessen östliche Abdachung dic Grenze zwischen Epirus und Thessalien führt, nach dem 1134 hoch gelegenen Orte Chaliki herab; den folgenden Tag bestieg ich den Oxya ${ }^{2}(1900 \mathrm{~m})$, kam Abends im Dorfe Kastania $(10 \% 0 \mathrm{~m})$, welches seinen Namen ron den zahlreich in der Umgebung wachsenden echten Kastanien erhalten hat, an und gelangte entlang des Kastaniotikos- und Peneios-Thales nach Kalabaka $(250 \mathrm{~m}) .{ }^{3}$ Am 21 . Juli durchquerte ich die thessalische Ebene bis Volo, wo ich mich am 22. nach Athen einschiffte. ${ }^{4}$

Wie aus dicser Darstellung ersichtlich, sind die den Osten und Vesten Thessaliens begrenzenden Gebirgszüge, also Olymp, Ossa und Pelion einerseits und die Pinduskette andererseits, wie auch ein ziemlicher Theil der thessalischen Ebene, wenn auch sicherlich bei weitem noch nicht vollständig, so doch zu einem ansehnlichen Theile von Fachmännern durchforscht worden. Dagegen ist das an der südlichen Grenze sich hinziehende Othrys-Gebirge und das im Norden gelegene, den Pindus mit dem Olymp verbindende Chasiagebirge noch gänzlich unbekannt. Dass die Durchforschung dieser Gebirge, insbesondere aber des letzteren wegen etwaigen Herabgreifens nördlicherer Typen von gánz besonderem Interesse wäre, ist sclbstredend.

Das Ergebniss dicser verschiedenen Expeditionen in pflanzengeographischer Beziehung ist, dass die Vegetation Thessaliens in den unteren Regionen der mediterranen Flora zuzuzällen ist, und dass die Hochgebirgsflora im Allgemeinen den Charakter der griechischen Berge trägt. Allerdings ist diese gemischt mit einer ansehnlichen Zahl endemischer und einer nicht minder erheblichen Zahl baltisch-mitteleuropäischer Formen.

Im Folgenden möchte ich nur noch in cinigen Zeilen die Vegetationsverhältnisse des von mir bereisten Gubietes schildern.

Das Aspropotamos-Thal, welches bei dem Orte Chaliki etwa $1100 \mathrm{~m}$ hoch liegt und daselbst durch die Abhänge des Peristeri im Westen und durch jene des Oxya im Osten gebildet wird, beherbergt eine Flora, wie etwa die in entsprechender Höhe gelegenen Partien des Sülabhanges des Peristeri. Die Unterlage ist hier gleichfalls Kalk. Abics Apollinis, Wachholder, Pirus communis, Cormus sanguinea, einzelne Ostrya carpinifolia und am Flusse selbst Salix incana und purpurea repräsentiren den Baumwuchs. Daplute olcoides und eine Menge von Pleris aquitima bilden die nächst untere Schichte. Von Kräutern und Stauden wären zu ervähnen: Helleborns cyclophyllus. Capsclla grandiflora, Silene caesia, Hypericum per-

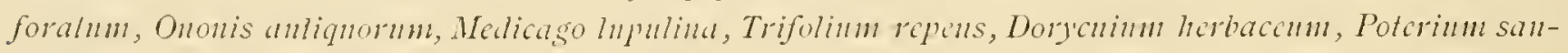

1 Deutsehe botanische Monatsschrift $1890-91$.

2 Nicht zu verwechseln mit dem gleichnimigen, an der Grenze des nordwestliehen Aelolien, in der heutigen Landschaft kira-

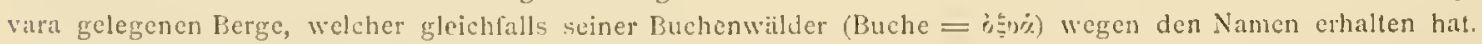

3 Die hier angelührten Ilöhenangaben verdanke ich Herrn Oberstlieutenant H. Hartl, Vorstand der geodätischen Absheilung in k. k. militär-geographischen Institute in Wien.

4 Die Reise machte ich mit dem Artilleric-Lieutenant der griechischen Amee Hern 1'. Chrysanthopoulos und mit Herrn C. Schwarzenberger. 


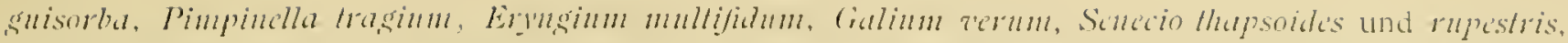

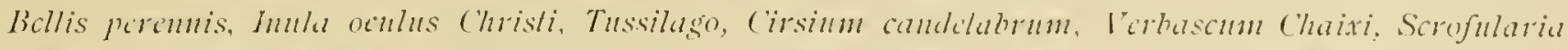

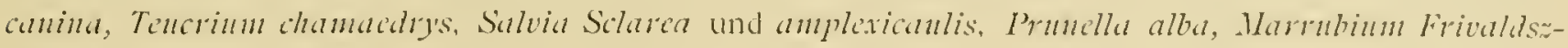
kyanum, Vicromeria juliana, Euphorbia myrsinites, Melica ciliata, Dactylis glomerala, Cynosurus edhinalus etc.

Dieselbe Vegetation weist auch noch die unterste Region des Oxya auf. In einer Seehöhe ron $1200-1300 \mathrm{~m}$ jedoch, in welcher Sandstein dem Kalke Platz macht, ändert sich der Typus der Vegetation wie mit einem Schlage. Mächtige, bis auf dic höchsten Erhebungen reichende Buchenwälảer und ausgedehnte Wiesen charakterisiren dieselbe. Im dichten, schattigen, unterholzlosen Bestande der ersteren begrüssen uns heimatliche Arten, wie Rubus idacus, Fragaria resca, Epilobium montanmm, Galium rolun-

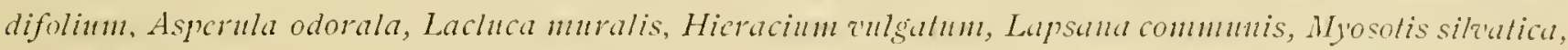
Poa nemoralis, ferner Arabis irepidipoda. Saxifraga Hcuffelii und Calaminlhagrandiflova. In Holzschlägen gedeihen Dianthus inodorus, Trifolinm alpestre. Rubus tomentosus, Artcmisia absinthimm, Campamula expansa. Digilalis ferraginca, Limaria minor. Clinopodiun und Trtica dioica. Auf den Wiesen finden sich als tonangebende Arten Veralrum Lobliamm und stellenweise grosse Strecken beherrschend Pteris aqui-

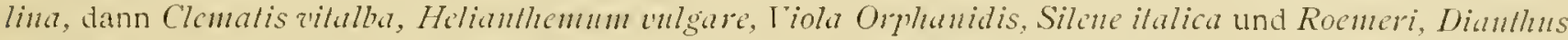
delloides, Linum callaticum, Hypricum bathatum var. pindicolum, Goranimm asphodeloides, Medicago falcala und Inpulina, Trifolimm arrense und Orphanidenm, Lolus cormiculalus, Lallymus pratensis, Spivalea filipendula, Agrimonia cupatoria. Erynginn campestre, Bellis perennis, Carlina acanlhifolia, Leontodon hastilis, Campamula flagellaris, T'rbascum Samaritanii, Scrofularia Siopolii, Calamintha alpina, Armeria canescens, Blilum bonus Henricus, Scirpus Holoschocmus. An den Waldrändern stehen einzeln Acer monspessulanmu, Pranus pscudoarmoniaca, Pirms malus, commumis und amygdaliformis. Crataegus flabcllata.

Gleichwie am Westabhange reicht auch am Serpentin führenden südöstlichen die Buchenregion bis etwa auf 1200 m Höhe herab, wird aber hier durch einen ausgedehnten Bestand von Föhren ${ }^{1}$ abgelöst. Das Unterholz in demselben wird durch Juniperus o.ycedrus gebildet. Der Rand des Föhrenwaldes ist dicht umsäumt von mächtigen Buxusbüschen. Hier und da steht auch ein Eibenbaum. Im Übrigen ist die Flora hier ziemlich arm. Charakteristisch ist das Auftreten zweier neuer endemischer Arten, der Silene Hansskncchtii und der Campanula Hamkinsiana, welche, wie es scheint, an die Serpentin-Unterlage gebunden sind. Häufig sind Helleborus cyclophyllus, Aly'ssum argcnlenm, Heliantlomum anlgare. Trifolinm arzense, Lotus corniculatus, Rubus ulmifolius, Fragaria resca, Erguginm campestre, Chamalence afra, Echinm italicum, Prunella alba, Calmmintha alpina, Pinguicula hirliflora. Euphorbia myrsinites, Scirpus Holoschoimus, Cynosurus echinalus, Plcris aquilina.

Bei dem Orte Kastania (1000 $m$ ) treten schüttere Eichenwälder (Quercus pubescus) auf, welche stellenweise mit unbewaldeten steinigen Abhängen abwechseln. In den ersteren wachsen: Cislus incunus, Sitcue Niederi und italica, Tunica illyica, Hypricum perforatum. Trifolium Pignantii und aricuse, Dorycuimm

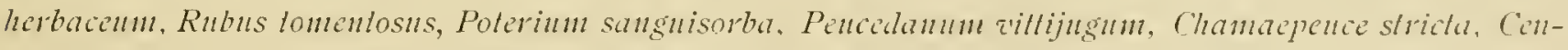
lamra Zuceariniana, Climopodium, Tencrimm polimm. Enplorbia myrsinites; auf letzteren zum Theile dieselben Arten, und ausserdem Tunica glumacia. Ononis antiquormm, Psorala, Rubus ulmifolius, Ruplemrum

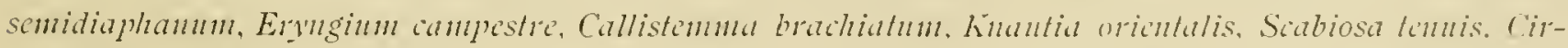

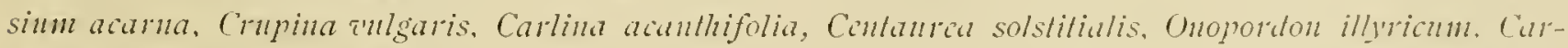

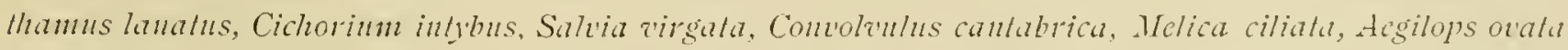
und einzelne Sträucher ron Olea und Pirms amysdaliformis. Die Ufer des hier fliessenden Kastaniotikos beherbergen: Galega officinalis, Elilobium hirsulum. Sambucus cbulus, Cirsium camdelabrum und siculum, Melissa officinalis. Der Peneios selbst, im Sommer ein leicht durchwatbares Flüsschen, stellenweise jedoch zu einem breiten, sandigen und steinigen Flussbette verbreitert, ist an einzelnen Stellen mit Platanenauen

1 Pinus nigricans oder P. Pallasiam. Die Art konnte nicht bestimmt werden, da das gresammelte Material in Verlust gerieth. 
Jicht bewachsen, in welchen man ab und zu wilde Reben antrifft. Auch Populus uigra. Ampisalus inmmmins, ferner Büsche von likex. Cionura und Plumbago finden sich hier vor.

Einen schon fast gänzlich mediterranen Charakter zeigen die malerischen Conglomeratfelsen von Kalabaka (250 $\mathrm{m}$ ), Aeginium der Alten, auf deren Kuppen die berïhmten Meteoraklöster thronen. Am Fusse derselben whichert Ouerus coccifera; dazwischen gedeihen Delpliminum hallerutum. Gypsophila polygonoides, Silone thessalonica. Alsine thessala, Achillea courtata, Cirsinm acama. Scolymus hispanicus. Echium italicum, Harrubium perghrinum, Ballota acelabulosa und die seltene Athamanta macelonica.'

Von Káabaka senkt sich almälig das Terrain und weitet sich bei Trikala zur einförmigen, fast völlig baumlosen, von einer ebenfalls rein mediterranen Flora beherrschten thessalischen Ebene aus. Einförmig nicht allein in der Configuration, sondern auch bezüglich ihrer Vegetation. Als Charakter-Pflanzen figuriren auf derselben Sinlymus hispanicus, Eryghinm irticum und Ammi l'isnağa, welche stellenweise grosse Strecken gelb, beziehtnngsweise bläulich oder weiss färben. Häufig treten auch noch auf: Hypericum crispum, Erynginm campestre. Onopordon illyricum. Eithinops microceplahlus, Nolobasis, Contaura salonitama und solstitialis, Acanthus spinosus und Marmbinm peregrimm.

\section{Aufzählung der beobachteten Arten.}

In dieser Aufzählung sind sämmtliche von mir beobachtete Arten, auch wenn dieselben schon für Thessalien von meinen Vorgängem festgestellt worden waren, aufgenommen. Möglichst reiche Standortsangaben sind ja für die Kenntniss der Flora eines Landes unbedingt wünschenswerth. Ausser diesen Arten sind weiters noch eine Anzahl angefüht, welche $\mathrm{H}$. Hartl, Vorstand der geodetischen Abtheilung im $k, k$. milit. geogr. Institute in Wien, auf dem Berge Tringia $(2195 \mathrm{~m}$ ), südlich von Kastania und Ch. Leonis auf dem Pelion und einzeinen Punkten der thessalischen Ehene im verflossenen Sommer gesammelt hatten.

1. Clematis flammula L. Sp. pl. p. 544 (1753).

In Gebüschen bei liastania.

2. Clematis vitalba L. Sp. pl. p. $5+4$ (1763).

An Heclien auf dem Oxya. Höhe $1300 \mathrm{~m}$.

3. Ranunculus concinnatus Schott, in Öst. bot. Wochenbl. V11, p. 182, (1857). - Cf. Hal. Beitr.z. F1. des Epirus, p. $10[226]$.

Auf dem Gipfel des Tringia (leg. Hartl).

4. Helleborus cyclophyllus Al. Braun Ind. sem. Bcrol. 1861, p. 14 pro var. H viridis; Boiss. Fl. or. 1, p. 61 (1867).

Auf dem Oxya bei Chaliki und Kastania.

5. Delphinium halteratum Sibth. et Sm. Fl. Graec. Prodr, I, p. 371 (1806).

Bei Kalabaka.

6. Barbarea vulgaris K. Br. in Ait. hort. Kew. ed. '2. IV, P. 109 (1812).

Var. macrophylla nov: var. (an spec.?)

Für den ersten Blick durch die aussergewölnnlich grossen Grundblätter, die sammt Stiel eine Länge von $35 \mathrm{~cm}$ erreichen und deren eirunder, besonders mächtig entwickelter Endlappen bis $10 \mathrm{~cm}$ lang und etwa ebenso breit ist, auffallend. Die Fruchttrauben sind verlingert, ihre Schoten durch die von der Axe winkelig-abstehenden Stiele aufrecht-abstehend, gerade, $25-30 \mathrm{~mm}$ lang, dick und mit einem verhältnissmässig kurzen Giriffel gekrönt. Die Blüthen sind unbekannt.

Wird vielleicht bei Vorhandensein eines reicheren Materiales als selbständige Art aufgefasst werden müssen. Die ebenfalls in Thessalien rorkommende $h$. arcuafa lar. braclycarpa Hausskn. Symb, ad flor. graec. in Nittheil. Thür. bot. Ver. 18\%3, p. 105, welche mir in einem schönen, von Heldreich aul der Hoch-

1 Conf. Hausskneeht in . Vittheil, des Thüring. bol. Vercins. Neue Fulge, V. Ileft, p. 110 (1893). 
ebene Neuropolis des dolopischen Pindus gesammelten Fruchtexemplare vorliegt und die ich der bereits bestehenden B. hrachycarpa Boiss. Wegen B. Ifausshnechtii nennen möchte, ist durch die alufrechtstehenden kleineren, mit längerem Griffel versehenen Schoten und die viel kleineren Blätter, wie die im Ganzen schmaichtigere 'lracht verschieden.

Auf dem Pelion oberhalb Volo (leg. Leonis).

7. Arabis crepidipoda Griscb. apul Pant. in Öst. bot. Zeitschr. XXlll, p. 268 (1873) et in Pant. Adnot. ad Flor. Herceg., Cernag. et Dalm. p. 90 (1874).

In Buchenwäldern des Oxya. Höhe $1700 \mathrm{~m}$.

Von der in der Tracht sehr ähnlichen Turritis slatra L. durch die einreihigen Samen in jedem Fache rerschieden.

$\mathrm{Ob}$ diese Art von A. pseudoturritis Boiss. et Heldr. specifisch zu trennen sei, ist bei dem geringen Materiale, das von diesen wenig gesammelten Arten in den Herbarien vorliegt, schwer zu entscheiden. Von A. psuloturritis liegen mir Exemplare von beiden bisher bekannten Standorten, rom Nalerogehirge und dem thessalischen Olymp vor, von welchen wohl nur sehr schwer A. crepidipoda durch die entferntgezähnten unteren Stengelblätter und die etwas längeren Blüthenstiele zu unterscheiden ist. Diese von Grisebach hervorgehobenen Unterscheidungsmerkmale dürften übrigens kaum durchgreifend sein, da die grundständigen Blätter beider Arten buchtig-gezähnt sind und diese Zahnung auch gelegentlich auf die nächststehenden Blätter übergreifen kann, und weil die Länge der Blüthenstiele auch nicht durchaus constant ist. Man wird in der Zukunft daher wahrscheinlich A. crepidipoda als Synonym zu A. psendoturritis zu stellen haben. Wenn ich dennoch die Oxya-Pflanze als $A$. crepiclipoda anführe, so geschieht dies aus dem Grunde, weil die Exemplare in der Mehrzahl die erwähnten Merkmale aufweisen.

8. Stenophragma Thalianum L. Sp. pl. p. 665 sub_Arabide (1753); Čel. Prodr. Fl. Böhm. p. 445 (1807). Auf Wiesen des Oxya. Hóne $1800 \mathrm{~m}$.

9. Aubrietia gracilis Sprun. in Boiss. Diagn. Pl. or. Ser. I, Nr. 1, p $7+(18+2)$.

Auf dem Tringia (leg. Hartl).

10. Alyssum argenteum Vitm. Summa plant. IV, p. 430 (1790). - A. mmale W. et K. Pl. rar. Hung. I, p. 5 (1802).

Am Rande von Föhrenwäldern des Oxya oberhalb Kastania.

Es ist dies offenbar dieselbe Pflanze, Welche Haussknecht in demselben Gebiete vorfand und die von ihm in Nitth. Thür. bot. Ver. 1893, p. 113, als A. cllorocarpum von A. murale mit dem Bemerken, sie unterscheide sich von dieser Art dureh die Biennität und den Mlangel steriler Sprosse, abgetrennt wurde. Meiner Anschauung nach ist sie jedoch von dem formenreichen A. argenleum nicht verschieden.

11. Capsella grandiflora Bory et Chaub. Fl. Pelop. Nr. 1017, t. 38 sub Thlaspide (1838); Boiss. Diagn. Pl. or. Ser. I, p. $76(1842)$.

Am Fusse des Oxya bei Chaliki.

12. Cistus incanus L. Sp. pI. p. 524 (1753).

Am Rande der Eichenwảlder bei Kastania.

13. Helianthemum vulgare Gaertn. De fruct. I, p. 371 (1788).

Auf dem Oxya. Höhe $1600 \mathrm{~m}$.

14. Viola Orphanidis Boiss. Fl. or. 1, p. 464 (1867). - V. Nicolai Pant. in Öst. bot. Zeitschr. XXIII, p. 4 (1873).

Auf Wiesen und am Rande der Buchenwälder des Oxya in grosser Menge. Höhe $1600 \mathrm{~m}$.

15. Silene flavescens W. et K. Pl, rar. Hung. II, p. 191 (180J).

Var. thessalonica Boiss. et Heldr. Diagn. Pl. or. Ser. 1I, Nr. 1, p. 74 pro spec. (1853); Rolibb. Monogr. Gatt. Silene, p. 146 (1868).

Auf Conglomeratfelsen bei Kalabaka. 
Ich führe S. thessalonica nach dem Vorgange ron Rohrbach als Varietït der S. flar'scens an, da die Unterscheidungsmerkmale heider Arten unconstant und Übergangsformen Zwischen beiden rorhanden sind, bei welchen letzteren man oft in Verlegenheit ist, ob man sie zu der einen oder anderen Art stellen soll. Die Endglieder dieser Formenreihe unterscheiden sich zwar sehr gut von einander, indem typische S. flavescens eine geringere Belileidung des Stengels und der Blätter aufweist und ihre Blüthen einzelnstehend und langgestielt sind, während typische S. thessalonica von dichtstehenden weissen Härchen grau ist und ihre kurzgestielten Blüthen eine armblüthige (2-5) traubige Inflorescenz bilden; diese Nerkmale finden sich jedoch nur in extremis. Es gibt eine ganze Reihe von Formen, die diese beiden Endglieder verbinden, so z. B. solche, bei denen der Stengel einblüthig, aber der Blüthenstiel kurz, nur so lang als der helch ist, dann wieder solche mit "- Z3blüthigem Stengel mit relativ längeren und auch oft verschieden langen Blüthenstielen u. S. W. In eben dem Masse ist auch die Belieidung variabel und combinirt sich verschiedenartig mit den verschiedenen obigen Formen. Die ron Sinten is und Bornmüller Iter turc Nr. 816 von Lawra und vou der Insel Thasos sub Nr. 585 ausgegebenen, von mir in öst. bot. Zeitschr. 1892, p. 368 und 414 als S. thessalonica aufgezähiten Pflanzen sind auch als Nittelformen und kaum als Schattenformen der S. favescens, wie Haussknecht in Symb. ad fl. graec., p. 48, will, aufzufassen, da sie eine dichte Bekleidung. wie S. thessalonica, dagegen cine infrorescenz wie S. flavescats, aulweisen. Auch unter diesen sind schon einzelne Exiemplare, deren Stengel zweiblüthig sind, mit relativ kurzen Blüthenstielen.

16. Silene italica L. Sp. pl. p. 593 sub Cucnbalo (1762); Pers. Syn. 1, p. 498 (1805).

In Buchenwäldern des Oxya. Höhe $1500 \mathrm{~m}$.

\section{Silene Schwarzenbergeri Species nova.}

Sectio Paniculatae Boiss. Fl. or. 1, p. 57 t. Basi suffutescens, multicaulis, inferne griseo-pubescens, superne viscidula; caulibus erectis simplicibus, bi-vel abortu unifloris; foliis radicalibus parvis obovato-spathulatis mucronatis, caulinis minimis anguste linearibus; floribus erectis; calyce pubescente, viridi-vittato, bisi umbilicato, etiam sub anthesi elongato-clavato, dentibus ovatis obtusiusculis, albomarginatis ciliatis; lamina livida, in lacinias oblongo-spathulatas bipartita, unguibus glabris, coronac laciniis lanceolatis acutis: lilamentis glabris, antheris purpureis; capsula oblonga, carpophoro subbreviore.

Stengel $20 \mathrm{~cm}$ hoch, Blattspreite $6 m \mathrm{~m}$ lang, $4 m \mathrm{~m}$ breit, Blüthen $2 \mathrm{~cm}$ lang.

Die hier beschriebene, meinem lieben Reisebegleiter C. Schwarzenberger gewidmete Art hat den dicken, knorrigen Wurzelstock, wie etwa S. paradoxa und ist vielleicht noch am nächsten mit S. Sicbcri Fenzl verwandt, welche sich aber durch dichterlilzige, länglichkeilige, nichtbespitzte Grundblätter, viel grössere weisse Blüthen, bewimperte Nügel und abgerundete Krönchenzähne von ihr unterscheidet.

Am Felsen des Oxya oberhalb Chaliki, sehr selten. H. $1500 \mathrm{~m}$.

18. Silene Niederi Heldr. in Boiss. Diagn. IPl. or. Ser. II, Nr. 6, p. 32 (1859).

In Eichenbeständen bei liastania, selten.

19. Silene linifolia Sibth. et Sm. Fl. Graec. Prodr. I, P. 301 (1806).

Auf dem Pelion bei Makriniza (leg. Leonis).

20. Silene Roemeri Friv. in Flora XIX, p. 439 (1836).

Auf dem Oxya. Höhe $1500 \mathrm{~m}$.

21. Silene fruticulosa Sieb. in DC. Prods. I, p. 376 (1824).

Am Fusse des Oxya bei Chalilit.

22. Silene multicaulis Guss. Pl. rar. p. 172 (1826).

Auf dem Pelion (leg. Leonis).

In einer breitblätterigen, der S. gemislifolia Hal. öst. bot. Zeitschr. XLIl, p. 369, sich nähernden Form, welche letztere ich übrigens nach neuerlich vorgenommener Untersuchung als Varietït zu S. multicaulis Guss. stellen möchte. 
23. Silene Haussknechtii Heldr. apud Hausskn. Symb. ad n. grace. in Mitth. Thüring. hot. Ver. V', p. i) (189:3).

Am Rande der löhrenwiilder des Oxya oberhall Kastania. Höhe l’no m.

24. Saponaria officinalis L. Sp. pl. p. $408(1759)$.

Bei Kalabaka.

25. Gypsophila thymifolia Sibth. ef Sm. Fl. Gracc. Prodr. 1, p. 282 (1806).

An Fusse des Pelion bei Volo.

26. Gypsophila polygonoides Willa. Sp. pl. II, I, p. 690 sub Cucubalo (1799). - C. ocellata Sibth. et Sm. Fl. Graec. Prodr. I, p. 281 (1806).

Auf Conglomeratfelsen bei Kalabaka.

Ist bezüglich der Anzahl der Blüthen in jedem Knäuel, der Anordnung der letzteren und der Blüthengrösse, wie auch bezüglich der Bekleidung bedeutenden Abänderungen unterworfen. Alle mir bekannten griechischen Formen jedoch, welche bei genauerem Studium eines reichen Materiales übrigens zum Theile als selbstündige Arten aufgefasst werden dürften, besitzen als gemeinsames Merkmal die geknäuelten, fast sitzenden oder kurgestielten Blüthen und die kurze, mehr weniger sammtartige Behaarung aller Theile, im Gegensatze zu G. thymifolia Sibth. et Sm., welche eine lange, abstehende, zottige Beharang, am Stengel z. B. länger als der Durchmesser desselben, aufweist und deren Inflorescenz eine lockere Trusdolde darstellt. Ob die ungefleckte Platte der Kronblïtter, welches Merkmal Sihthorp und Smith besonders hervorheben, nur dieser Art eigen ist. oder auch etwa bei der einen oder anderen form der C. poly'sonoides vorknmmt, vermag ich nach trockenem Material nicht zu entscheiden.

27. Tunica illyrica L. Mant. p. T0 sub Saponaria (1767); Boiss. 11. n. I, p. 520 (1867).

In Eichenwällern des Oxya unterhalh Kiastania.

28. Tunica thessala Boiss. Diagn. Pl. or. Ser. 1, Nr. S, p. 63 (18ti1).

Auf dem Pelion bei Makriniza (leg. l,eonis).

20. Dianthus glumaceus Bory et Chauh. in Exp. scient. Mor. 111, 2. p. 340 (1832).

In Eichenwïldem bei Lastania.

30. Dianthus haematocalyx Boiss. et l1eldr. Diagn. Pl. or. Ser. 11, Nr. I, p. 65) (185i3).

Var. pruinosus Boiss. 1. c. Nr.6, p. 28 pro spec. (1859); Poiss. Fl. nr. 1, p. 503 (1867).

Am Fusse des Pelion bei V́no, loc. class.

31. Dianthus deltoides I. Sp. pl. p. $898(175.3)$.

Cimasplätze auf dem Oxya. Höhe $1500 \mathrm{~m}$.

32. Dianthus viscidus Bory et Chaub. in Exp. scient. N101. 111, 2, p. 119 (1832).

Auf dem Pelinn (leg. Leonis) und in Eichenwïldem bei liastania.

33. Dianthus inodorus 1. Sp. pl. p. 413 pro var. D. caryoplyylli $(17.3 .3)$; Kern. lil. exp. Austro-hung. Nr. it3 (1882). - D. silvestris Wulf. in Jacq. Coll. I. p. 237 (1786).

Auf Felsen des Oxya. Höhe $1600 \mathrm{~m}$.

34. Velezia rigida 1. Sp. pl. P. 332 (1753).

Bei Kastania.

35. Alsine thessala Species nova.

Sectio Tryphaneae Boiss. Fl. or. 1, p. 676. Perennis, caespitosa, glaucovirens, pedicellis exceptis glaberrima; caudiculis laxiusculis adscendentibus; foliis lineari-subulatis, subincurris, uninerriis, axillis fasciculiferis; caulibus superne nudis, corymbiferis, rarius uninoris; pedicellis capillaribus, parce et hrevissime glanduloso-hirtis, calyce 3-5 plo longioribus; sepalis oblongo-lanceolatis, acuminatis, trinervis, albomarginatis; petalis albis, integris, oblongo-lancenlntis, acutis, calyee evidenter brevioribus; seminibus reniformibus, minute tuberculatis. 
Der A. attica Boiss. et Sprun. zunäichststehend, Welche aber cine robusterc Pflanze, mit kräftigeren Stengeln, rigideren Blättern und grösseren Blüthen ist. Ausserdem ist letztere in allen 'Theilen dichtdrüsig und hat dreinerrige Blätter.

Aul Conglomeratfelsen hei lialabaka und auf dem Oxya. Von Sintenis und Bornmüller auch aul Felsen im Thale Megarema bei I itochory am Fusie des Olymps gefunden (Iter turc. a. 1891, Nr. 1176 sul A. icrua var, acutipetala).

36. Alsine Gerardi Willd. Spec. pl. II, 1, p. 729 sub Armaria (1796); Wahlenb. Fl. Carp. P. 1:32 $(1814)$

An Felsen des Oxya. Höhe $1500 \mathrm{~m}$.

37. Alsine stellata Clarke Travels in var, contr. of Eur, Asia and Afr. 11, 3, p. 211 sub Cherleria (IS16); II al. Beitr. Fl. Epirus, p. 16[292].

Var. epirota Hal. l. c.

Auf dem Gipfel des Tringia bei Kastania (leg. Hal 1 l).

38. Linum catharticum L. Sp. pl. F. 281 (175:i).

tuf Wiesen des Oxya. Höhe $1500 \mathrm{~m}$.

39. Hypericum barbatum I a cq. 11. Austr. 1II, p. 33 (175.5).

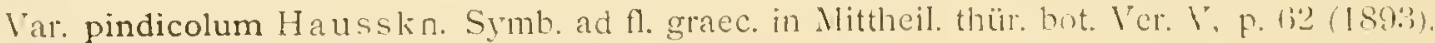
Unter Ciebüsch auf dem Oxya. Höhe $1500 \mathrm{~m}$.

40. Hypericum tetrapterum Fr. Nov. Fl. Suec. III, F. 94 (1S17).

Feuchte Waldplätze hei Kastania. Höhe $800 \mathrm{~m}$.

41. Hypericum perforatum L. Sp. pl. p. $785(17.793)$

Bei Chaliki und Kastania an Waldrändern.

42. Hypericum crispum L. Nant. p. 106 (1767).

An der thessalischen Bahn bei Karditza und Sophades.

43. Acer monspessulanum L. Sp. pl. p. 1056 (175.3)

Einzeln am Rande der Buchenwilder des Oxya.

41. Vitis silvestris Gmel. Fl. Bad. 1, F. 543 (1806).

In Gebüschen bei Kastania.

45. Geranium subcaulescens I. Her. in DC. Prodr. 1, P. (3.10 (182-1).

Auf dem Gipfel des Tringia (leg. Hartl).

46. Geranium asphodeloides Burm. Specim. bot. de Geran. p. 28 (17.6\%).

Auf dem Oxin oberhalh Chaliki.

47. Paliurus australis Gaertn. de fruct. I, P. 203 (1788).

Bei liastania.

48. Cercis siliquastrum 1. Sp. pl. p. $37+(1753)$.

Bei liastania.

49. Podocytisus caramanicus Boiss. et Heldr. Diagn. Pl. or. Ser. I, N1. 9, P. T (184(1). Buschige Pliitze bei Kastania.

50. Ononis antiquorum L. Sp. pl. ed. Il, p. $1000(1763)$.

Bei Chaliki und Kisstania.

51. Medicago falcata L. Sp. pl. P.779 (17.73).

Aul dem Oxy'a bei Chaliki.

52. Medicago lupulina 1. Sp. pI. P. 779 (175.3).

Aul dem (xya hei Chaliki. 
5i:. Trifolium alpestre 1. Sp. pl. ed. 2, p. 1082) (1703is)

Vatr. incanum Cies. in Griseb. Spic. kil. Rum. et Bithyn. 1, p. 25) (184:3).

IViesen aul dem Oxya. Höhe $1500 \mathrm{~m}$.

54. Trifolium Pignantii Fauché et Chalub. in Exped. scient. Mor. 113, 2. F. 219 (1832).

In Buchenwäldern des Uxya bei Chaliki und Oxa Despot, dann in Eichenwäldern bei Kastania.

55. Trifolium arvense 1.. Sp.pl. p. 769 (1753.)

Auf dem Oxya.

56. Trifolium Parnassi Boiss. et Sprun. Diagn. PI. or. Ser. 1, Nr. 2, p. $30(1843)$.

Aur dem Gipfel des Tringia (leg. Hartl).

57. Trifolium repens 1. Sp. pl. p. 767 (175i).

Am Fusse des Oxya bei Chaliki.

Var. Orphanideum Buiss. Diagn. P1. or. Ser. 11, Nr. 2, p. 17 pro spec (1856); Fl. or. 11, p. $11.5(1872$, Auf dem Oxya und Tringia.

58. Dorycnium herbaceum Vill. Hist. pl. Daluph. 111 , p. 317 (1789).

Bei Chaliki und Kastania.

59. Lotus corniculatus 1. Sp. pl. p. 765 (175:3).

Bei Chaliki am liusse des Oxya.

60. Colutea arborescens L. Sp. pl. P. 723 (1753).

Bei Lalabilia.

61. Galega officinalis L. Sp. pl. p. $714(1753)$.

Bei Kiastania.

62. Psoralea bituminosa 1. Sp. pl. p. 763 (1753)

Bei liastania.

63 Onobrychis gracilis Bess. Enum. pl. Volh. p. 74 (1822).

In Eichenwë̌ldern bei Kastania.

Ist durch längere Dornen an den Hülsen vom Typus verschicden. Weder zu (1. pindicola Hausskn.. noch zu O. graeca Hausskn. gehörig.

64. Lathyrus pratensis L. Sp. pl. p. $733(1753)$.

Auf dem Oxya bei Chaliki.

65. Amygdalus communis L. Sp. pl. p. $473(1753)$.

Bei Ralabaka.

66. Prunus pseudoarmeniaca Heidr. et Salt. in Boiss. Diagn. pl. or. Ser. 11, Nr.5, p. (16) (185̄ob).

Auf dem Oxya.

67. Spiraea filipendula 1. Sp. pl. p. $490(1753)$.

Auf Wiesen des Oxya. Jlöhe $1500 \mathrm{~m}$.

18. Rubus idaeus 1. Sp. pl. p. $4920(1753)$

In Buchenwäldern des Oxya.

69. Rubus ulmifolius Schott in 1sis 1818, p. 821

Bei Kastania und Claaliki.

70. Rubus tomentosus Burkh. in Rocm. neu. Mag. But. 1. p. 22 (1794).

Auf dem Oxya.

71. Fragaria vesca L. Sp. pl. F. 494 (1753)

Auf dem Oxya bei Chalili. 
72. Potentilla pedata Nestl. Momogr. Polent. p. 43 (1816).

V'ar. glabrescens Hausskn. Symb ad tl. grace. in Mittheil. 'Thür. bot. Ver. V, p. 92 (189:3). Auf dem Tringia (leg. Hartl).

73. Rosa glutinosa Sibth. el Sm. F!. Gracc. Prodr. I, p. :348 (1806).

Var. leioclada Christ in Boiss. Fl. or. suppl. p. 2222 (1888).

Auf dem Oxya. Höhe $1500 \mathrm{~m}$.

74. Agrimonia eupatoria L. Sp. pl. p. 448 (1753).

Auf dem Oxya.

75. Poterium sanguisorba L. Sp. pl. p. 914 (1753).

Bei Chaliki und Kastania.

76. Pirus Malus L. Sp. pl. p. 479 (1753).

In Waildern auf dem Oxya.

77. Pirus communis L. Sp. pl. p. $479(1653)$.

In Wäldern auf dem Oxya.

78. Pirus amygdaliformis Vill. Cat. hort. Strasb. p. 322 (1807).

Bei liastania und aul dem Oxya.

79. Crataegus orientalis Pall. in N. a. Bi cb. Fl. taul. Cauc. 1, p. 387 (1808).

F. flabellata Buiss. Fl. or. II, p. 66I (1872).

Waldränder auf dem Oxya.

SO. Epilobium hirsutum L. Sp. pl. p. 347 (1753).

Bachufer bei Kastania.

81. Epilobium mantanum L. Sp. pl. p. $348\left(1753^{\circ}\right)$.

Buchenwälder des Oxya. Höhe $1500 \mathrm{~m}$.

82. Lythrum salicaria L. Sp. pl. p. 146 (1753).

Bei lialabaka.

83. Herniaria parnassica Heldr. et Salt. in Bulss. Diagn. Pl. or. Ser. 11, Nr. 1, p. 45 (1853). Auf dem Gipfel des Tringia (leg. Hartl).

84. Sedum acre L. Sp. pl. p. 432 (1753).

Auf dem Gipfel des Tringia (leg. Hartl) und Oxya.

85. Sedum magellense Ten. Prodr. Fl. Nap. p. XX'1'1, 6 (1811).

Auf dem Gipfel des Tringia (leg. Hartl).

86. Saxifraga Heuffelii Schott. Anal. bot. P. 25 (1854).

in Buchenwälldern des Oxya. Höhe $1500 \mathrm{~m}$.

87. Peucedanum vittijugum Boiss. Fl. or. 11, P. 1018 (187').

Waldränder bei Kastania.

88. Athamanta macedonica L. Sp. pl. p. 253 sub Bulone (1753); Spleng. in Schult. syst. veg. VI p. $491(1820)$.

Aul Conglomeratfelsen bei Kalabaki. Schon von Formanek und Haussknecht daselbst angegebun.

89. Ammi Visnaga L. Sp. pl. p. 2t22 sub Danco (1753); L.am. dict. 1, p. 132 (1789). In der thessalischen Ehene bei hardiza und Sophades weite Strecken übereichend.

90. Pimpinella Tragium Vill. prosp. hist. pl. Dauph. p. 24 (1779).

Auf dem Oxya bei Chaliki.

01. Pimpinella peregrina L. Sp. pl. p. $26+(.753)$.

Bei Kinstania. 
92. Carum rupestre Boiss. et Ifeldr. Diagn. P1. or. Ser. 11, Nr. 2, p. $70(18.56)$.

Auf dem Gipfel des Tringia (leg. Hartl).

93. Bupleurum semidiaphanum Boiss. Diagn. 11. or. Ser. 11, Nr. 6, p.733 (1859).

Bei Kastania und am Fusse des Pelion bei Volo.

94. Eryngium multifidum Sibth. et Sm. Fl. Graec. Prodr. I, p. 175 (1806).

Am lusse des Oxya bei Chaliki.

95. Eryngium campestre L. Sp. pl. p. 233 (1753).

Bei Chaliki, Kastania, auf dem Oxya und in ler thessalischen Ebene.

96. Eryngium creticum Lam. Dict. JV, p. 754 (1897).

Bei Kastania und auf der thessalischen Bahn.

97. Cornus mas L. Sp. pl. p. 117 (1753).

Am Fusse des Oxya bei Chaliki.

98. Sambucus Ebulus L. Sp. pl. p. 269 (1753).

Bei Lastania und an der thessalischen Bahn.

99. Galium rotundifolium L. Sp. pl. p. 108 (1753).

In Buchenwäldern des Oxya.

100. Galium verum L. Sp. pl. p. 107 (1753).

Bei Chaliki.

101. Asperula odorata 1. Sp. pl. p. 103 (175:3).

In Buchenwïldern des Oxya.

102. Asperula longiflora W. et K. Pl. rar. Hung. 11, p. 162 (1805).

Var. condensata Heldr. ap. Wettst. in Bibliogr. bot. Heft 20, p. $59\left(189^{\circ}\right)$.

Auf dem Gipfel des Tringia (leg. Hartl).

103. Callistemma palaestinum 1. Mant. p. 117 sub kinanlia (1767).

Bei Kástania.

104. Scabiosa tenuis Sprun. in Boiss. Diagn. Pl. or. Ser. 1, Nr. 2, p. 114 (1843).

Bei Kastania.

105. Knautia orientalis L. Sp. pl. p. 101 (1753).

Bei Kilstania.

106. Senecio thapsoides DC. Prodr. VII, p. 301 (1838).

Am Fusse des Oxya bei Chaliki.

107. Senecio rupestris W. et K. Pl. rar. Hung. II, p. 136 (1805).

Bei Chaliki.

108. Anthemis tinctoria L. Sp. pl. p. 896 (1753).

Var. discoidea Boiss. Fl. or. III, p. 281 (1875).

Bei Kastania.

109. Achillea Neilreichii Kern. in öst. bot. Zeitschr. XXI, p. $1+1$ (1871). - A. punclutu'Ten. Prudr. Fl. Neap. p. L (1811) non Moench. Meth. pl. Marb. p. 603 (1794).

Auf dem Oxya oberhalb Kastania.

110. Achillea coarctata Poir. Enc. meth. suppl. 1, p.94 (1810). - A. compacla Willd. Sp. pl. III p. 2206 (1800) non Lam. - 4. sericea Janka in Linnaea XXX, p. 579 (1860).

An Conglomeratfelsen bei Kalabakit.

111. Chrysanthenum tenuifolium Kit. in Schult. Oestr. Fl. ed. 2, II, p. 498 (1814). - C. trichophyllum Boiss. Diagn. Pl. or. Ser. 1, N1. 4, p. 10 (1844). - Pyrcthrum trichophyllum Grieseb. Spic. Fl. 


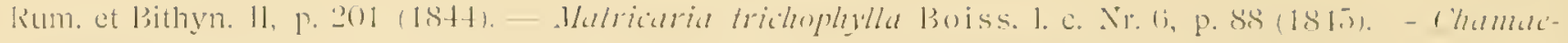

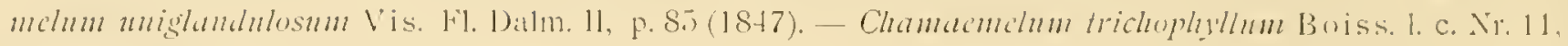
p. 21 (1849).

Yar. discoideum. - Ligulis mullis.

Auf dom Oxya oberhalb Kistania.

112. Artemisia absinthium L. Sp. pl. p. $848(1753)$.

Auf dem Oxyra.

113. Bellis perennis L. Sp. pl. p. 886 (1753).

Auf iem Oxyin.

11. Inula oculus Christi 1. Sp. pl. p. 881 (1753).

Auf dem Oxya.

115. Tussilago farfara L. Sp. pl. p. 865 (1753).

Auf Jem Oxya.

116. Echinops microcephalus Sibth. et Sm. Fil. Grace. Protr. 11. p. 209 (1813).

Häufig an der thessalischen Bahnstrecke.

117. Echinops graecus Mill. Dict. ed 8, Nr. 4 (1768).

Auf wüsten Plätzen bei Volu.

18. Carlina acanthifolia All. Fl. Pel. I, 1. 156 (1785).

Auf dem Oxya bei Chaliki und hastania.

119. Onopordon illyricum 1. Sp. Pl. p. 827 (1753).

Bei Kastania und an der thessalischen Bahn.

120. Silybum Marianum 1. Sp. pl. p. 238 sub Cardmo (175:3); Catertn. Fict. 1l. p. 37s (17!1).

An der thessalischen Bahn.

121. Chamaepeuce afra Jacq. Hort. Schoenbr. 11, p. 180 sub Carduo (17!9); DC. Prodr. VI, p. 659 $(1837)$.

Rei Chaliki am Fusse des Oxyá.

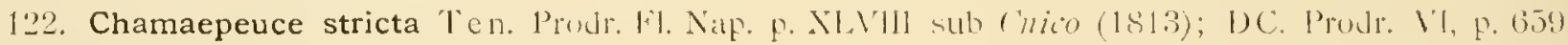
$(18: 37)$.

In Eichenwäldern bei liastania, selten.

123. Notobasis syriaca L. Sp. pl. p. 82:3 sub Carduo (1753): ('ass. 1)ich. scienc. nat. XXXY, p. 170) $(1825)$.

In der thessalischen Ebene.

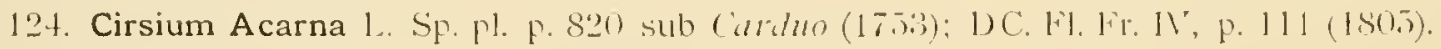

Bei Kastania und Kalabakia.

125. Cirsium candelabrum Giriseb. Spic. Fl. Rum. und Bithyn. 11, p. 251 (184t).

Auf Jem Oxya bei Chaliki und Kastania.

126. Cirsium siculum Spreng. Neu. Entdeck. III. p. 36 (1820).

Bei Kástania.

127. Carduus acanthoides 1. Sp. pl. p. 821 (17.33).

Var. thessalus Boiss. Fl, or. III, p. 518 (1875).

Bei Palacolinstro (leg. Leonis).

128. Carthamus lanatus L. Sp. pl. p. $8: 30$ (16.3i3).

Bei Kastania und Volu.

129. Cnicus benedictus 1. Sp. pl. p. $820(1753)$.

Bei Kastania. 
130. Centaurea macedonica Griseb. Spic. F1. Rum. et Pythin. 11, p. 2to pro var. C. pamiculala.

Bei liastania und Kalabaka.

131. Centaurea pelia DC. Prodr. V'1, P. 586 (1837).

Bei Voln am Fusse des Pelion (locus classicus).

1:3:2. Centaurea Zuccariniana D( . Prode. VT, p. 574 (18:37).

Bei Kastania und Kalabaka.

133. Centaurea salonitana Jis. in Ergänzhl. z. hotan. 7cit. p. 2:3 (1829).

Bei Kalabalia und an der Bahn bei Sophades.

1:34. Centaurea solstitialis L. Sp. pl. p. 917 (1753).

Bei Kastania, Kalabaka, Sophades und Volo.

13i). Lactuca muralis L. Sp. pl. p. 797 sub Pronanthe (17.5:3); Gaertn. De liruct. 11, t. 158 (1791).

In Buchenwäldern auf dem Oxya. Hölıe $1400 \mathrm{~m}$.

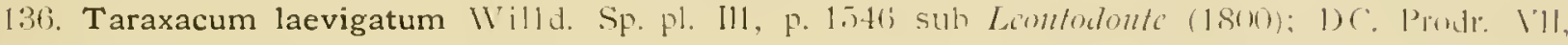
p. $149(1813)$.

Aul dem Tringia (leg. Hartl).

137. Hieracium vulgatum Fr. Now. ed. 11, p. 258 (1898).

In Buchenwäildern auf dem Oxya. Höhe $1400 m$.

138. Hieracium pannosum Boiss. Diagn. Pl. ol. Ser. I, Ni. 4. P. 32 (184t).

Aul Felsen des Oxya. Höhe $1400 \mathrm{~m}$.

138. Hieracium Heldreichii Boiss. Diagn. Pl. or. Ser. 11, N1. 3, p. 102 (185i).

Auf felsen des Oxya. Höhe $1400 \mathrm{~m}$.

139. Crepis Columnae Ten. Syll. Pl. Nap. p. 398 sul Hicracio (1831); Fruel. in Dr. Prodr. V11, P. 167 (18:38).

Aut dem Gipfel des Tringia (leg. Hartl).

140. Leontodon hastilis L. Sp. pl. ed. 2, p. 1123 (1763).

Auf Wiesen des Oxya.

141. Scolymus hispanicus L. Sp. pl. p. 813 (175.3).

Pei Kastania, Kalabaka, entlang der thessalischen Bahn his Vulo gemein.

1.2. Cichorium intybus L. Sp. pl. F. $81: 3$ (175:3).

Bei Chaliki und liastania.

143. Lapsana communis 1. Sp. pl. P. $811(17,53)$.

In Buchenwäldern des Oxya. Höhe 1400 m.

14. Campanula flagellaris Hal. in Beitr. z. F1. Epirus, p. 30.

Auf Wicsen des Oxya. Höhe $1400 \mathrm{~m}$.

145. Campanula foliosa '1'en. Fl. Nap. Prodr. P. XYl (1811).

In Buchenwäldern auf dem Oxya. Höhe $1400 \mathrm{~m}$.

146. Campanula athoa Boiss. Diagn. Pl. or. Ser. II, Nr. '3, P. 110 (1850).

Auf lem Pelion (leg. Leonis).

147. Campanula spathulata Sibth. et Sm. Fl. Graec. Prodr. i, p. 132 (1806).

Auf dem Gipfel des Tringia (leg. Hartl) und auf dem Oxya.

14. Campanula Hawkinsiana Heldr. et Hausskn. in Heldr. Herb. graec. norm. Nir. S56 (18S. $)$.

An Serpentinfelsen des Oxya oberhalb Kastania häufig.

Da die Diagnose Jieser noch nicht beschriebenen. Art in Hausskn. Symh, ad Fl, wrace. in Bäilue zu erholfen steht, begnïge ich mich mit der eiufachen Aufzähilung derselben. 
149. Campanula expansa Fris. in Flora 1836, I1, p. 434.

In Buchenwäldern des Oxya. Höhe $1500 \mathrm{~m}$.

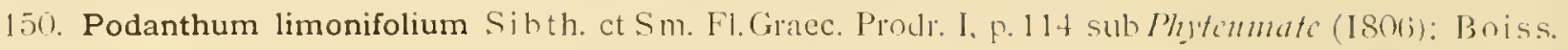
Fi. or. III, p. 163 (1875)

Auf dem Gipfel des 'Tringia (leg. Hart1).

151. Olea europaea L. Sp. pl. p. $8(1753)$.

Bei Kastania.

152. Cionura erecta 1. Sp. pl. p. 213 suh (ymancho (17.3): Grisch. Spic. Fl. Rum. et Bithyn. 1I, p. 09 $(1.844)$.

Bei kalabaka.

15\%. Convolvulus cantabrica L.. SP. pl. p. 158 (175\%).

Bci Kastania.

1.7. Echium italicum L. Sp. pl. p. $139(1753)$.

Bci Kastania und Kalabaka.

155. Alkanna Pulmonaria Grisch. Spic. Fl. Rum. ct Bithỵn. II, p. (1) (1844).

Auf Conglomeratfelsen bei Kalabaka, selten.

156. Myosotis silvatica Hoffm. Deutschl. PI. 1, p. (1) (1791).

In Buchenwäldern auf dem Oxya.

157. Verbascum Samaritanii Hcldr. in Boiss. Diagn. Pl. or. Ser. Il, Nr. (5, P. 127 (I853).

Am Rande der Buchenwälder aul dem Oxya. Höhe $1500 \mathrm{~m}$.

158. Verbascum Heldreichii Boiss. Diagn. Fl. or. II, Nr. 3, P. 117 (1854i).

Bei Palaeokastro (leg. Leonis).

159. Verbascum Chaixi V'ill. Hist. pl. Dauph. II, p. 491 (1787).

Am Fusse des Oxya bei Chaliki.

160. Scrofularia Scopolii Hloppe in Pers syrn. 11. p. $160(1807)$.

Am Rande der Buchenwä̈lder des Oxya.

161. Scrofularia canina L. Sp. pl. p. (321 (175\%).

Bci Chaliki.

162. Digitalis ferruginea L. Sp. pl. p. 122 2 (175:3).

In Holzschlïigen auf dem Oxya.

103 Linaria minor L. Sp. pl. p. 617 sub Antirlhino (175:3): Desf. Wi. Atl. 11, p. 46 (1798).

Auf lem Oxya.

16it. Acanthus spinosus L. Sp. pl. p. (639) (175i3).

Bci Kastania und an der thessalischen Bahn.

16.5. Vitex agnus castus L. Sp. pl. p. $(93,38$ (175i3).

Pei Kalabaka.

166. Verbena officinalis L. Sp. pl. p. $20(175,3)$.

Bei Kastania.

167. Teucrium Chamaedrys L. Sp. pl. p. 56.) (1753).

Bei Chaliki.

168. Teucrium Polium 1.. Sp. pl. p. $566(1653)$.

Bei Kastania.

169. Salvia Sclarea L. Sp. pl. p. 27 (1753).

Bei Chaliki. 
170. Salvia amplexicaulis Lam. Ill. 1, p. 68 (1791).

Bei Chaliki.

171. Salvia virgata Ait. Hort. Kew. I, p. 39 (2789).

Bei Kastania.

172. Prunella vulgaris 1.. Sp. pl. p. 600 (1753).

Bei Chalili.

173. Prunella laciniata L. Sp. pl. p. 600 plo var. P. vulgaris (1753); L. Sp. pl. ed. 2, p. 837 (1763). Bei Chaliki und kastania.

174. Betonica scardica Gijeh. Iter. Rumel. Il, p. Is9 sub Stachyde (1839); Spic. Fl. Rum. et Bithy'n.

11. p. 136 (1844). - b. gracea Bo iss. et Sprun. Diagn. P1. or. Ser. I, Nr. 5, p. 27 (I844).

In Eichenbeständen des Oxia oberhalb Kastania.

175. Stachys penicillata Heldr. et Sart. in Buiss. Diagn. PJ. or. Ser. 11, Nr. 4, P. 37 (185i).

Iuf dem Pelion (leg. Leonis).

176. Phlomis samia L. Sp. pl. p. 585 (1753).

Iuf dem Pelion (leg. Leunis)).

17. Ballota acetabulosa L. Sp.pl. p. 584 sub Marrubio (1753): Benth. Labiat. p. 595 (1832-1836). Bei Kalabaka.

178. Marrubium peregrinum L. Sp. pl. p. 582 (1753).

Bei Kastania, Kalabaka und an der thessalischen Bahn bei Sophades.

17\%. Sideritis scardica Griseb. Spic. Fl. Rum. et Bithyn. 1I, p. 144 (1844).

Auf tem Pelion (leg. Leonis).

180. Clinopodium vulgare I.. Sp. pl. p. 587 (1753).

In Buchenwäldern des Oxya.

181. Calamintha grandiflora L. Sp. pl. p. 592 sub Melissa (1753); Mocnch. meth. P. 408 (1794).

In Buchenwäldern des Oxya. Höhe $1300 \mathrm{~m}$.

182. Calamintha alpina L. Sp. pl. p. 591 sub Thymo (1753); Lam. Fl. fr. II, p. 394 (1778).

Auli dem Oxya.

183. Micromeria juliana L. Sp. pl. p. 567 sub Saluria (1753); Benth. Labiat. p. 378 (1832-1836). Bei Chaliki.

184. Micromeria cremnophila Boiss. et Heldr. in Boiss. Fl. ol. IV, p. 570 (1879).

Auf Conglomeratfelsen bei Kalabaka.

185. Origanum viride Boiss. Fl. or. IV, p. 5īl provar. O. unlgaris (1879).

Bei lialabalia.

186. Thymus teucrioides Boiss. et Sprun. Diagn. PJ. ol. Ser. J, Nr. 5, p. 15 (J84t).

Auf dem Gipfel des Tringia (leg. Ha l"tl).

187. Pinguicula hirtiflora Ten. Fl. Nap. III, p. 18 (1811).

Feuchte Stellen des Oxya oberhalb Lastania.

188. Lysimachia atropurpurea L. Sp. pl. p. 147 (1753).

Bei Kalabaka.

189. Plumbago europaea L. Sp. pl. p. 151 (1753).

Im Lande des Peneios bei Kalabaka.

190. Armeria canescens Host. Fl. Austr. I, p. 407 sub Stalice (1827); Host. in Ebel. Armer. gen. p. 28 (1S40).

Auf Wiesen des Oxya. Hölne $1500 \mathrm{~m}$.

(v. Haläcsy.) 
191. Plantago lanceolata L. Sp. pl. p. 113 (1753).

Bei Chaliki.

192. Blitum bonus Henricus L. Sp. pl. p. 218 sub (henopodio (1753); C. A. Mey. in Led. Fl. Alt. I, p. 11 (1829).

Auf dem Oxya zuweilen grosse Strecken dicht überziehend.

193. Chenopodium botrys L. Sp. pl. p. 219 (1753).

Im Sande des Peneios bei Kalabakia.

194. Daphne oleoides Schreb. Ic. et descript. pl. min. eugn. dec. 1, p. 13 (1766).

Auf dem Tringia (leg. Hartl) und Oxya bei Chaliki.

195. Buxus sempervirens 1. Sp. pl. p. 983 (1753).

Im Thale des Flusses Kastaniolilius am Rande von Föhrenwäldern cinen ausgejehnten Bestand bildend.

196. Euphorbia myrsinites L. Sp. pl. p. 461 (1753).

Auf dem Tringia (legr. Hartl) und Oxya.

197. Ficus carica L. Sp. pl. p. 1059 (1753).

Bei Kalabaka.

198. Platanus orientalis L. Sp. pl. p. 099 (1753).

An den Ufern des Peneios und scincr Nebenflüsse.

199. Urtica dioica L. Sp. pl. p. nst (1753).

Auf dem Tringia (leg. Hartl) und Oxya.

200. Ulmus campestris L. Sp. pl. p. 225 (1753).

Bei Kastania.

201. Fagus silvatica L. Sp. pl. p. 998 (1753).

Auf dem Oxya dichte Wälder bildend. Höhe $1000-1500 \mathrm{~m}$.

202. Castanea sativa Mill. Gard. dict. ed. 1 (1768).

In Buchenwüildern bei Kastania.

203. Quercus pubescens Willd. Sp. pl. W , p. 450 (180.)).

Aul dem Oxya und bei Kastania ausgedehnte Bestände bildend.

204. Quercus Farnetto 'Ten. Cat. Nap. 1819, p. 65.

Bei Kístania.

205. Quercus coccifera L. Sp. pl. p. 995 (1753).

Bci Kastania und Kalabaka.

206. Ostrya carpinifolia Scop. Fl. carn. ed. 2, 11, p. 2.4 (1772).

Am Fusse des Oxya bei Chaliki.

207. Carpinus duinensis Scop. Fl. Carn. ed. 1I, 2. p. 243 (1772).

Bei Kastania.

208. Populus nigra L. Sp. pl. p. 1034 (1753).

An den Ufern des Peneios bei Kalabaka.

209. Salix alba L. Sp. pl. p. 1021 (1753).

An den Ufern des liastaniotilos.

210. Salix purpurea 1. Sp. pl. p. 1017 (1753).

An den Ufern des Aspropotamos bei Chaliki.

211. Salix incana Schrank. Bair. Fl. I, p. 230 (1789).

Mit voriger. 
212. Asphodeline lutea 1. Sp.pl. p. 30 s sub Asplodelo(1753); Reichenb. Fl. germ. exc., p. 116 (1830). Bei Challiki.

213. Veratrum Lobelianum Bernh. in Schrad. Neu. Journ. 11, 2-3 Stück, p. 356 (1808).

Auf Wiesen des Oxya. Höhe $1300 \mathrm{~m}$.

214. Scirpus Holoschoenus L. Sp. pl. p. 49 (1753).

Auf dem Oxya.

215. Beckmannia erucaeformis L. Sp. pl. p. 55 sub Phalariale (1753); Host. Gram. Austr. 111, p.5 (180,5). Bei Palaeokastro (leg. Leonis).

216. Phleum commutatum Gaud. Agrost. heli. I, p. 40 (1811).

Auf dem Tringia (leg. Hartl).

217. Alopecurus Gerardi All. F1. Ped. 11, p. 232 sub Phleo (1785); Vill. Hist. pl. Dauph. 11, p. 66 (1787).

Auf dem Tringia (leg. Hartl).

218. Cynosurus echinatus L. Sp. pl. p. i2 (1753).

Auf dem Oxya.

219. Dactylis glomerata L. Sp. pl. p. 71 (1753).

Auf den Oxya.

220. Melica ciliata L. Sp. pl. p. 66 (1753).

Bei Chaliki und Kastania.

221. Poa nemoralis L. Sp. pl. p. 69 (1753).

In Buchenwäldern des Oxya.

222. Poa alpina 1. Sp. pl. p. 67 (1753).

Var. parnassica Boiss. Fl. or. V, p. 605 (1884).

Auf dem Tringia (leg. Hart1).

2223. Haynaldia villosa L. Sp. pl. p. 81 sub Seculi (1753); Schur Lin. pl. Trans. p. 807 (1866).

Bei Kastania.

224. Aegilops ovata L. Sp. pl. p. 1050 (1753).

Bei Kastania.

225. Phacelurus digitatus Sibth. et Sm. Fl. graec. Prodr. I, p. 71 sub Rolbollia (1806); Griscb. Spic.

Fl. Rum. et Bithyn. 11, p. $42+$ (184t).

Bei Palaeokastro (leg. Leonis).

226. ? Pinus Pallasiana Lamb. Pin. ed. 2, p. 11 (1828).

In Kastaniotikosthale oberhalb Kastania. Bestände bildend.

227. Juniperus oxycedrus L. Sp. pl. p. 1038 (1753).

Auf dem Oxya bei Chaliki und Kastania.

228. Juniperus foetidissima Willd. Sp. pl. IV, p. 853 (1805).

Bei Kastania.

229. Taxus baccata 1. Sp. pl. p. 1040 (1753).

Auf dem Oxya oberhalb Kastania einzeln.

230. Pteris aquilina L. Sp. pl. p. 1075 (1753).

Auf dem Oxya.

231. Asplenium septentrionale L. Sp. pl. p. 1068 sub Atrostic/2o 1753): Hoffm. Deutschl. F1. I1, p. 12 (1791).

Auf Serpentinfelsen des Oxya oberhalb Kastania. 


\section{Flechten.'}

Das Substrat der flechten bilken: Kieselrollsteine theikweise mit cinem Kalküherzuge von Kalabaka, Kalkconglomerat von Kalabakit und Buchenrinde vom Oxy•a.

23:. Parmelia caperata Ach. Meth. p. 216. - Lin. Spec. plant. P. 1147 sub Lich.

Sterilis. Thallus centroversus papillis crebris dense vestitus.

Rullstein ron lialabaka.

233. Lecanora subfusca Ach. Un. p. 293. - Lin. Suec. p. 400 sub Lich.

Var. glabrata Ach. Univ. p. 393. - Syn. argenlata Ach. sec. Nyl. Scand. p. 160.

Auf Buchenrincle vom Oxya.

234. Lecanora (Sect. Aspicilia) concreta Schiir. Spic. p. 73.

Thallus $\mathrm{kHO}$ non mutatur.

Substeril aber ausgebreitę auf Kalk von Kalabaka.

235. Lecanora (Sect. Aspicilia) trachytica Mass. Ric. p. 4. - Arn. Flor. 1887, p. 150.

Thallus KHO rubet, apothecia nondum evoluta. Pycnides areolis convexis, distinctius cinereo-rufescentibus immersae, supra dilute rufescentes. Sterigmata subsimplicia, basidia majora. Pyenosporac $7-10 \% \lg , 1-1 \cdot 2$. It. rectac vel raro levissime curvatae.

Auf Kiesel von Kalabaka.

236. Lecidea parasema Arn. Jura. Scpar. p. 165. - Ach. Prodr. p. 6.t p. p. - Forma migulosu Arn. Jur. Sep. P. 166. - Ach. Univ. p. 176 p. p.

Auf Buchenrinde des Oxya.

237. Buellia maritima. - Syn. Calolechia marilima . Mass. Sym. p. 51.

Thallus magis caesio-cinereus quam Mass. exs. n. 271. Cactera quadrant. Sporae $8 \cdot 7-11$ p. $\lg$, (j) -8 . 1t., late ellipticae vel subrotundae.

Auf dem Roltstück von Kialabaka.

238. Rhizocarpon geographicum 1) C. Fl. franc. II, F. 365. - Lin. Spec. plant. I, p. lociz sub Lich.

Neben Nr. 237.

239. Verrucaria glaucina Ach. Univ. p. 675. - Syn. l'err. sulfuscolla Nyl. Scind. p. 2271.

Var. griseoatra krplli. Lich. Bay. p. 234.

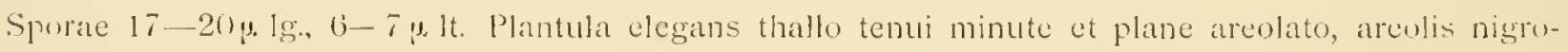
cinctis. Juxta adest planta loco magis obumbrato areolis turgidioribus, majoribus et minus conspicue nigro limbatis.

Neben Nr. 2:37 und 2'38.

240. Verrucaria (Sect. Lilhoicea) macrostoma 1) ul. in Fl. franç. II, p. 319.

Sporae $25-33 \mu \mathrm{lg}$., $16-19 \mu \mathrm{lt}$.

Auf kalk von Kalabaka.

241. Tichothecium erraticum Mass. Symm. p. 94.

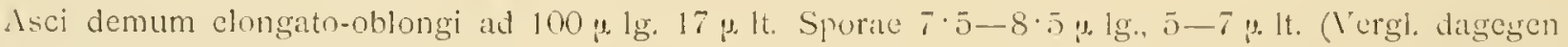
Rab. Crypt. k1., I. Bu., II. Abth. p. 3.50.

Auf dem Thallus von Nr. 234.

1 Bcarbeitct von J. Steiner. 
'212. Tichothecium pygmaeum lirb. L'at. p. 167.

l'all. ectanosporum inzi. Neosymb. p. 16.

sporac fuscosecntes non constrictac.

l'erithecien hecritenweise aul dem 'Thallus von Nr. 2:il.

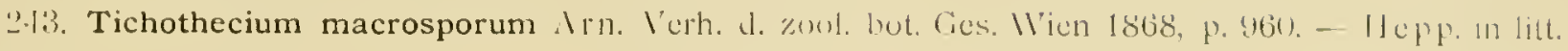
ald Ain.

Sporitc $20-25 \mu . \lg ., 7-\$ \mu .1 t$.

Aulf dem Thallus von Khizoc. geogralh.

\section{Verzeichniss der Gattungen.}

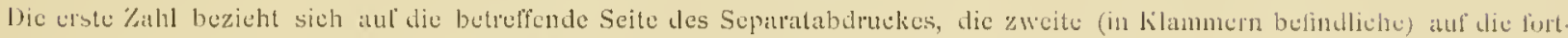
laufende l'aginirung des Bandes der Denkschriften der kais. Alademie.

\begin{tabular}{|c|c|c|c|}
\hline Seile & Campunula $\ldots 15\left[\frac{\text { Seite }}{479]}\right.$ & Echinops...... $14[4 \overline{78}]$. & · Lylhrum \\
\hline diamthus .....16 [480] & Capsella ..... $7[471]$ & Echimm .......16[480] & Marmbium ...17/48 \\
\hline Acir ......... . 10 [17t] & Cardums......14[478] & Enilobinm . . . . 12 [478] & Medicago \\
\hline Acliblle'c ...... 13 [477] & Carlinu....... 14[478] & $\ldots 13[477]$ & Melica........ . 19|18 \\
\hline Acsilops ... . . . 19 [483] & Carpinus .....18 [482] & Euplotoria & Micromerial ...17 [48 \\
\hline Asrimonia . . . 12 [476] & Carthanums....14 [+78] & Fagus ........18[180] & $M$ \\
\hline Alkamma ....... $16[480]$ & Carum ......13 [47 & Ficus ........ $18[480]$ & $N_{c}$ \\
\hline 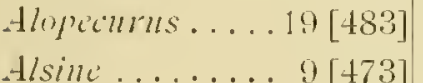 & Castanea......18 & Fragaria... & Olea \\
\hline Alsinc .... & Centanrea.....15 14 & Gialega. & On \\
\hline $\begin{array}{l}\text { Alyssum . . . . . } 7[471] \\
\text { dmmi ....... } 12[176]\end{array}$ & $\begin{array}{l}\text { Corcis ........10 [4 } \\
\text { Chamaepinci...14|4 }\end{array}$ & Galinm. & On \\
\hline Amygulalus .... 11 [475] & Chinopodium ... 18 [480] & Geramiln & $n \ldots$ \\
\hline Hullumis ....13[+77] & Chugsanlhemum $13[477]$ & Gypsopli & Origanu \\
\hline drabis.......7 74 & Cichorinm ....15 447 & dial ....19[483] & 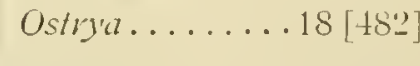 \\
\hline Armeria ......17 [t & Cionmma .....16 [480] & liemmm . . $7[4$ & $P c$ \\
\hline lisia..... & Cirsinm ......11 $[478$ & Helleborus .... 61470 & 76 \\
\hline 'ula......13 [t & Cistus ...... $7[47$ & ria .....12 $[476]$ & dammm ....1 \\
\hline ine $\ldots . .19\left[\frac{1}{2}\right.$ & Clematis......6 $6[470]$ & $m m \ldots .12$ & lurus \\
\hline mimm .....19[t & Clinopodium....17|181 & $H_{3}$ & (2) \\
\hline dlhamamla.....12 [476] & $\ldots \ldots \ldots 1+[478]$ & Inula . . . . . . 14[47 & Plilonis... \\
\hline Aubrielia. & Colutea ......11 [175] & Juniperus . . . . $19[483]$ & Pintpinclla .. \\
\hline Ballota ... & Contoliulus ....16 [480] & Finauliar & \\
\hline . $6[470]$ & Cormus ...... $13[t$ & & Pinlls . \\
\hline 3eckmanmia ....19[483] & Cratagens... & & Pirlis . . \\
\hline Bellis...... & Crepis........15 [479] & $\begin{array}{l}\text { Lat } \\
\text { Lat }\end{array}$ & Plantago... \\
\hline Bctomica... & Cynosurus .. & $\begin{array}{l}\text { Lathyras. } \\
\text { Lecallora. }\end{array}$ & Platamiss.. \\
\hline blitum $\ldots$ & Dactylis ......19[183] & Lecida . . . . & Pou ..... \\
\hline Bucllia... & Daplune.......18 [to & Letontorion . & Podanthum . . . $16[180$ \\
\hline lenrll|ll & Dilplinimm $\ldots o b[40]$ & Linuria... & Podocylisus.....10 [47. \\
\hline wtis .... & Diunthus..... 9 [473] & Lil & Populus.. \\
\hline intha & Digilalis ......10 Lte & Lotus. . . . . . . . & Potentilla. \\
\hline allistemma & Dorycuinm ....11 [4t & Lysinachia .... & Polcrimm \\
\hline
\end{tabular}




\begin{tabular}{|c|c|c|c|}
\hline & & & \\
\hline Irmmilla ...... $\overline{7} \mid \overline{481}$ & $.13|+73|$ & $.11|+78|$ & Tussilugo \\
\hline I'runus ......11|+75| & Salpenturia & Spiracal. & Clmms. \\
\hline Psomalia ......11 $[475\rceil$ & Sarifiasa. & Stacleys & Crtica. \\
\hline$\ldots \ldots \quad \ldots 19|+83|$ & Scabiusal . . & Stenopleragmal & Telo:ia. \\
\hline (Guctus ..... 18 [482] & $.19[483]$ & $.15[+79]$ & leralrum \\
\hline Rounumiulus ... u $|+70|$ & Scolymus & Taxus .......19|483] & I'erbuscum ... \\
\hline Rliracurpon . . . 20 [-18t] & Sorofulario & Tulurinu ... & Terboula \\
\hline Rosid . . . . . . 12 $\lfloor+76 \mid$ & $.12\lfloor+76\rfloor$ & Tlymmus...... 17 [481] & Verracaria ....2014i \\
\hline kubus ........11 $|+75|$ & Suncion ....... 13 $|476|$ & Tichothecium & Viola..... \\
\hline $2:]$ & $.17[481]$ & Trifolinm ... & ritex...... \\
\hline Sulviva . . . . . 16 1480$]$ & Sïlen ... & Tunical & litis \\
\hline
\end{tabular}

Erklärung der Abbildungen.

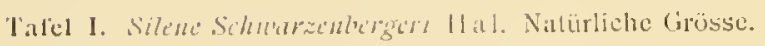

Tafel II. Alsiaz lhessaha Hal. Natürliche Grüsse. 


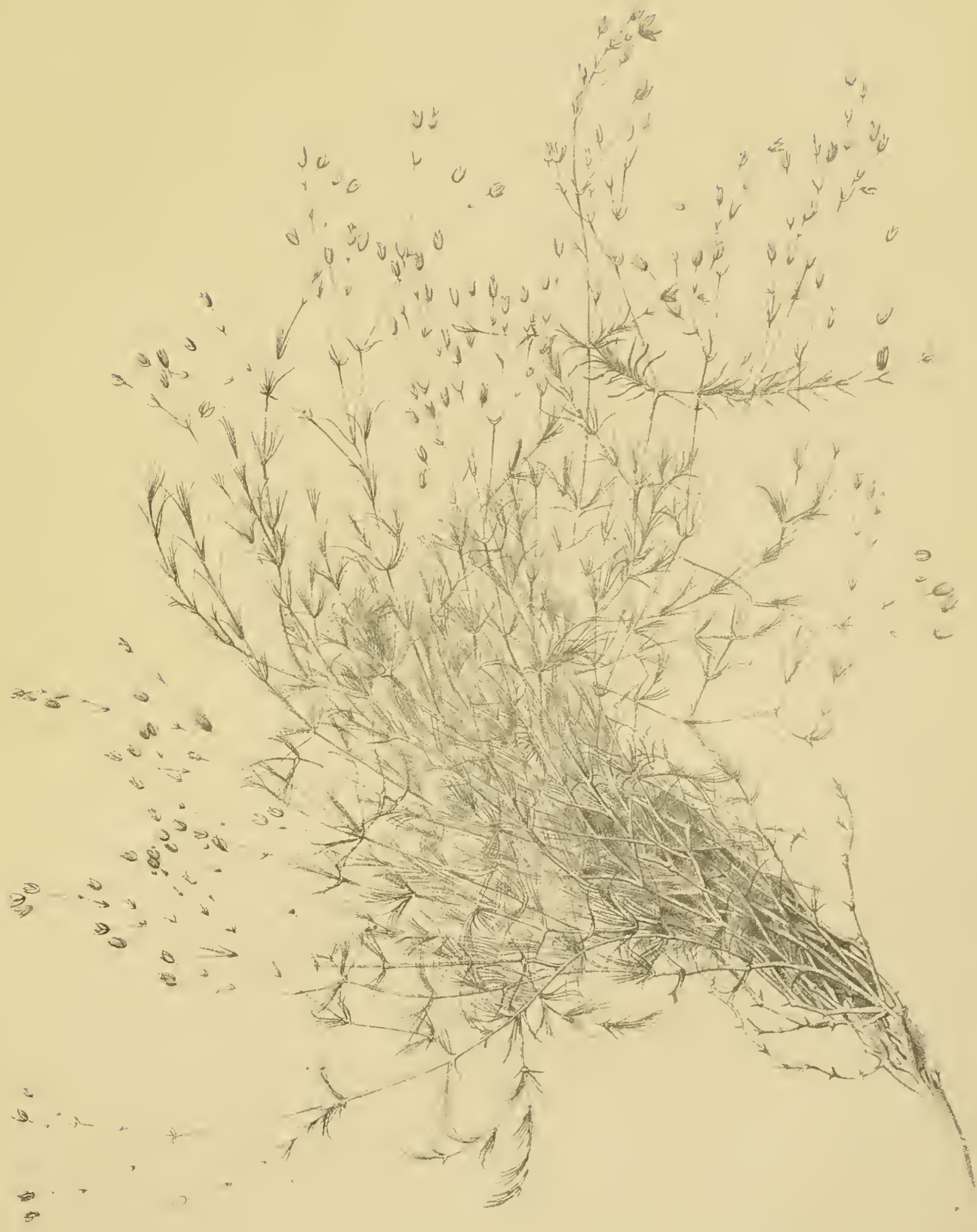



E.v. Halácsy: Beitrag z.ur Flora von Aetolien u. Acarnanien.

Tal' II.

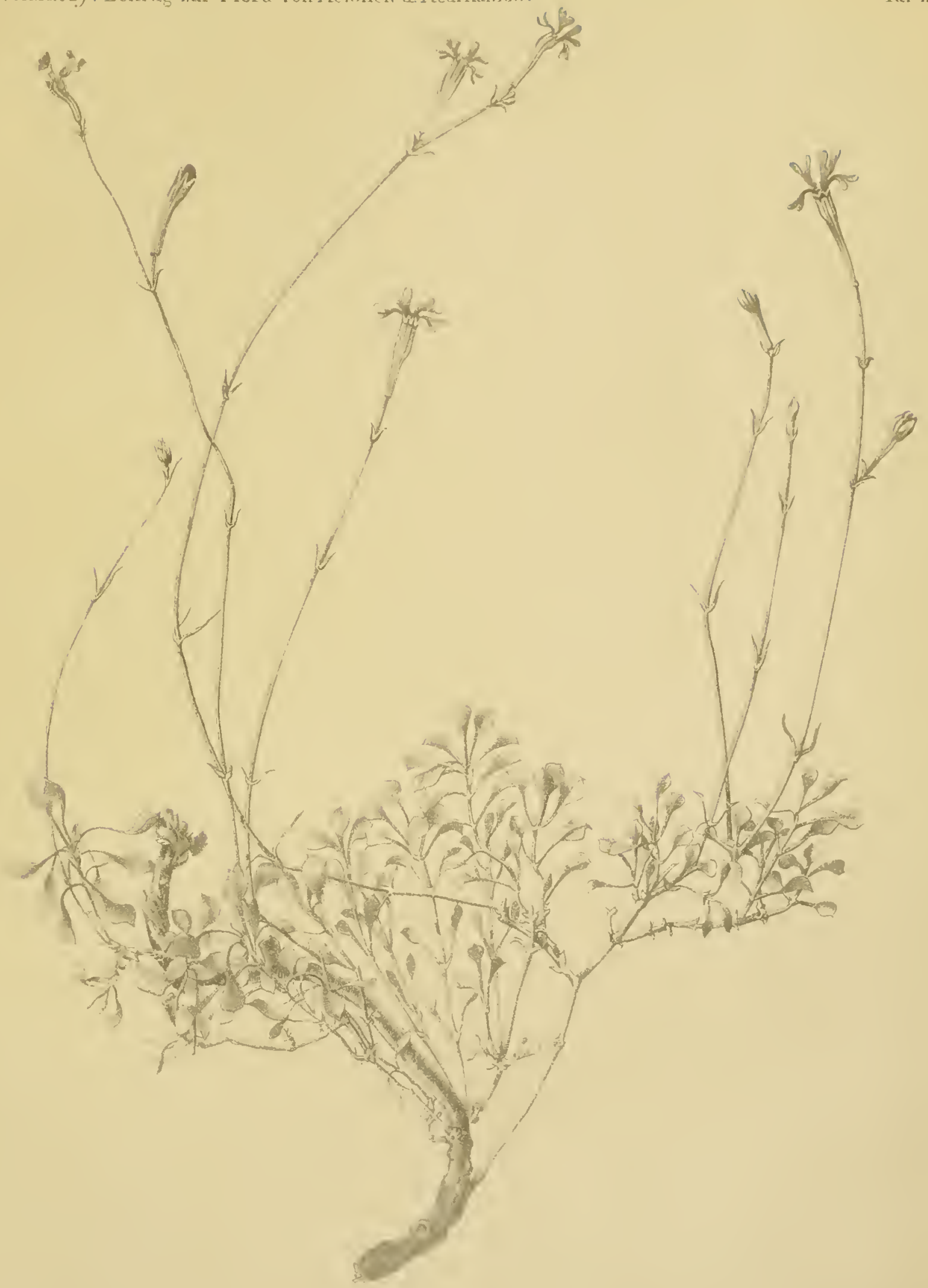



E. v. Halácsy.

\section{BOTANISCHE ERGEBNISSE}

EINER IM AUFTRAGE DER HOHEN KAISERL. AKADEMIE DER WISSENSCHAFTEN UNTERNOMMENEN FORSCHUNGSREISE

IN GRIECHENLAND.

IV. BEITRAG

ZUR

\section{FLORA VON ACHAIA UND ARCADIEN.}

VON

Dr. EUGEN v. HALÁCSY.

BESONDERS ARGEDRUCKT AUS DHM LXI. BANDE DFR DFNKSCHRIFTEN DER MATHENATISCH-NATURWISSFNSCHAFTIICHFN CLASSIE DFR KAISFRLICHFN AKADEMIE DER WISSFNSCHAFTEN.

WIEN I 894.

AUS DFR KAISERLICH-KÖNIGLICHEN HOF. UND STAATSDRUCKEREI.

IN COMMISSION BEI F. TEMPSKY.

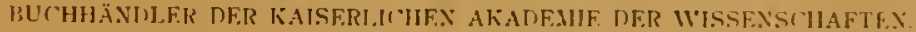

Preis: I fl. $25 \mathrm{kr} .=2 \mathrm{Mk} .50 \mathrm{Pfg}$. 



\title{
BOTANISCHE ERGEBNISSE
}

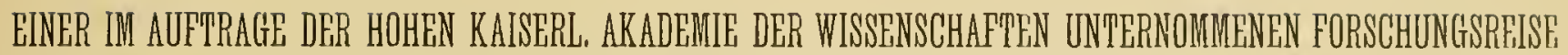

\author{
IN GRIECHENLAND.
}

\author{
IV. BEITRAG
}

ZITR

\section{FLORA VON ACHAIA UND ARCADIEN.}

$\operatorname{lON}$

\section{Dr. EUGEN v. HALÁCSY.}

\section{WIEN I 894.}


- 


\section{BOTANISCHE ERGEBNISSE}

EINER IM AUFTRAGE DER HOHEN KAISERL. AKADEMIE DER WISSENSCHAFTEN UNTERNOMMENEN FORSCHUNGSREISE

IN GRIECHENLAND.

IV. BLITRAG

ZUR

\section{FLORA VON ACHAIA UND ARCADIEN \\ $\operatorname{lox}$}

\section{Dr. EUGEN v. HALÁCSY.}

VORGELEGT IN DER SYTZUNG AM 2.'. MA! 18:4.

\section{Allgemeines.}

Die den nördlichen Theil des Peloponnes bildende Provinz Achaia mit dem angrenzenden Arcadien ist, gleich der ganzen Hatbinsel, ein Gebirgsland, welches im Norden jäh in den Golf von Corinth abfällt und deren höchste Erhebungen, wie der Panachaicon (1927 m, heute Voidia), der Olenos (2224 m, Erymanthos der Alten), der Chelmos (2355 $m$, Aroania der Alten) und die Kyllene (2374 $m$, heute Ziria) zu den bedeutendsten Gebirgsstöcken des Landes gehören.

Wo immer her man vom genannten Golfe in das zum grössten 'Theile aus kalk bestehende Gebirge eindringt, durchquert man einen schmalen, von Gebirgsbächen durchschnittenen und von sandigen Vorhügeln bedeckten Strich Landes, auf welchem ausgebreitet die Elemente einer, auf weite Strecken durch ansehnliche Weinculturen verdrängten Mediterranflora angetroffen werden. Diese beherrscht das ganze Gebiet, wie auch fast ausschliesslich die niedriger gelegenen Partien im Inneren des Landes, setzt auch zum grossen Theile die Vegetation der höher befindlichen Tannenregion zusammen und sendet selbst cinzelne Vertreter in die Region der griechischen Hochgebirgsflora.

Wie in den anderen Ländern des Mittelmeerbeckens, gliedert sie sich auch hier in einzelne Formationen, welche im Folgenden in Kürze geschildert werden sollen.

Als besonders charakteristischer Typus der mediterranen Flora ist auch hier in erster linie die Formation der immergrünen Buschwälder oder Macchien zu erwähnen. Sie treten in kleineren oder grösseren Beständen entlang der ganzen Küste auf, überziehen aber auch grössere Strecken, wie z. B südlich von Patras an der nach Chalandritza lührenden Strasse. Der Hauptmasse' nach bestehen sie aus dem Erdbeerbaume, Pistacia Lentiscus, MLytus. Phyllirea und Erica arboria. zwischen welche Arten einzelne Ölbäumchen, Rhus cotinus, Calycotome villosa oder Cercis siliquastrum sich eingesprengt vorlinden, häufig umrankt von der kletternden, mit zahllosen duftenden, weissen Blüthen besüeten Rosa sempervirens. In dem durch diese Arten gebildeten, stellenweise undurchdringlichen Dickichte, welches hier durchschnitt-

1 Leh führe hier, wie auch in Folgenden, nur die von mir heobachteten Arten an: auf Vullstïndigkeit kïnnen daher diese Aulzählungen keinen Anspluch erheben. 
lich einen geringeren Höhenwachsthum zeigt, als z. B. am Golf ron Arta oder auf den dalmatinischen Inseln sind keine günstigen Verhältnisse gegeben zur Entwicklung eines reicheren Niederwuchses; dagegen bieten die freieren Stellen oder die Ränder derselben hiezu die besten Bedingungen. Dieser Niederwuchs wird, hauptsächlich durch Anthyllis Hermamniae, Genista acanthoclada, Rubus nlmifolins. Potcrinn spinosum, Hypericum empetrifolinm, Sparlinm juncem, Cistns incanus, Osyris und Thymus capitalus gebilclet. Auch Qucrus coccifera ist stellenweise ein Bestandtheil desselben. Die unterste Schichte besteht aus einer ganzen Reihe von Stauden- und Krïuter-Arten, wie: Papaver Rhocas, Hirschfeldia alpressa, Alsine globnlosa, Limum spicatum, Althaca pallida, Ononis mollis und pubescens, Mclilolus neapolitana, Trifolium Chrrlevi, stellatum, pallidum und campestre, Bonjeania hirsuta. Psoralea. Vicia salaminia. Callistemma palucstinum. Kuantia hybrida. Hclichrysum italicum, Calcndula artensis, Crepis foctida, Picris panciflora, Hedypnois cretica, Campanula ramosissima, Convolvulus temuissimus, Tencrimm polinm, Salvia peloponnesiaca, Siderilis pumpura, Micromeria juliana, Plantago psyllium und lagopus, Cynosurus echinatus, Lóelevia phlcoides, Acgilops triaristala, Brachypodium distachyon.

Eine zweite Formation, welche hier bezüglich ihrer Ausdehnung allerdings von geringerer Bedeutung ist, ist jene der Meerstrands-Föhre (Pinus halepcnsis), welche an einzelnen Punkten der Küste in stark gelichteten Beständen auftritt. Die von zahllosen Cycaden umschwärmten Bäume mit ihren dünnen Nadeln und über mehrere Jahre an den Ästen verbleibenden Zapfen geben der Landschaft ein eigenartiges Gepräge. Der Niederwuchs in derselben besteht nahezu aus denselben Arten, wie in den Macchien, welche stellenweise in ihrer Gesammtheit in dieselbe eindringen.

Diese Formation steht in lebhaftem Contraste mit der oft unmittelbar an sie sich anschliessenden Formation des Oleander's, welche sich haptsächlich in den grobgerölligen Inundationsgebieten der Flussmündungen sich ausbreitet und den ganzen Sommer hindurch mit ihrer Blüthenpracht das Auge entzückt. Einzelne Platanen und mehr minder dichte Gebüsche von I'itcr agnuts castus und ron Weiden sind die last einzigen Mitbewohner dieser prïchtigen Formation.

Jene Areale der untersten Region, welche nicht durch die erwähnten Formationen occupirt sind und auch zum Weinbau nicht verwendet werden, wie der grössere Theil der den Gebirgen im Landesinneren vorgelagerten Sandhügel, sind baumlos oder doch nur mit vereinzelten Feigen- oder Olivenbäumen bewachsen. Sie sind im Sommer den sengenden Sonnenstrahlen preisgegeben, beherbergen aber dennoch selhst im Juni noch, in welchem Monate die Nielerschläge bereits gänzlich sistiren, eine aus einer verhältnissmässig reichen Anzahl von blühenden Stauden und kriütern zusammengesetzte Vegetation. Diese weist einen heideartigen Charakter auf, und es wiilen als wichtigere Arten derselben folgende anzuführen: Papaner

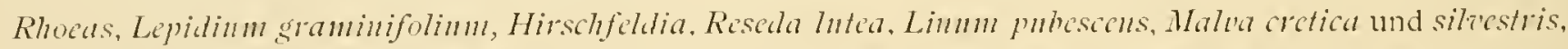
Hyprianm crispum. Erodium malacoides und cicutarium. Trifolimm scabrum, anginstifolium, phy'sodes

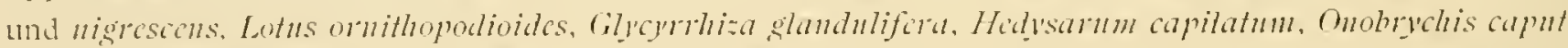

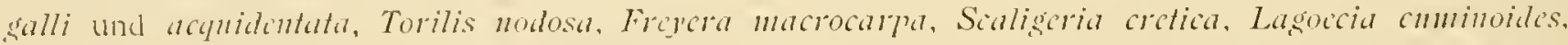

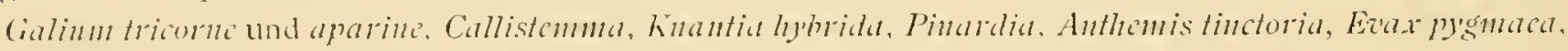
Asterisius aquaticus, Atractylis cancellatu. Cyuara cardmuculus. Gulactites tomentosa, Silymm. Notohasis,

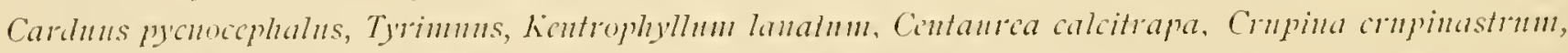

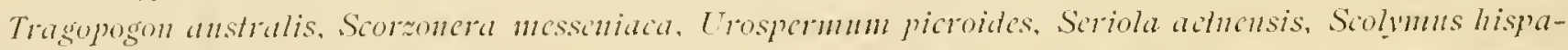
nicus, Campanula ramosissima. Chlora perfoliata. Anchusa lybriala, Echium italicum und plantagincum, Verlascum rigialum, Trixago apula, Enfragia viscosa, Orobunche minor. Acanthus spinosus. Salvia virgalu,

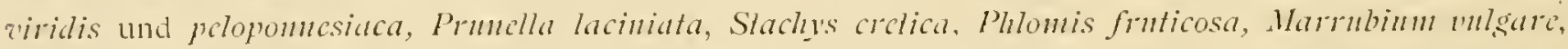
Sideritis purpurea, Anagallis cocrulea, Planlago Coronopus und Bellarli, Atriplex Halimus, Rumex conSlomeratus, Lrtica dioica, Oplury's commla. Scilla maritima. Armm itulicum, Imperata, Dactylis hispanica, Cynosmrus echinatns, Bromus malritensis, sterilis und intermedins, Hordemm hulbusum, Gandinial, Acgilops orata und triaristata. An Gräben: Rannuculus traclycarpus. Trifolium resupinatum, Lythrum Gracfferi, Hclosciadium nodiflormm. Ocnanthe incrassans, Samolus, Alisma Plantago. Cyperns longus, Scirpus mari-

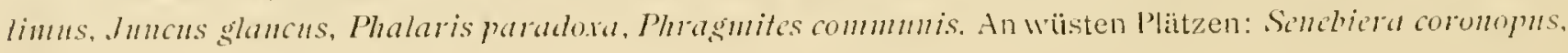


Sispmbrium polvectatium und officinale, Momordica, Lanthimm strumarimm, Matricaria chamomilla, lerbena officinalis.

Von häufiger cultivirten Bäumen wurden beobachtet: Robiniu pscudoacacia, Melia Azcdorach. Schinus molli, Sambucus nigira, Juglans, Ailanthus, Encalyplus, Mespitus japonica, Ficus, Cupressus. Amygedalus communis, Ligustrum japonicum, ritrus malica und aurantimn. Moras alba und nigra, Salix babylonica, Vicotiana glanca, Punica granatum. Zu Zäunen wird oft Opuntia und Agar'c verwendet.

Zu den wichtigsten Pflanzenformationen der unteren Region muss noch jene der Kcrmeseiche (Quercus coccifera) gezihltt werden. Diese, offenbar der widerstandsfihigste Rest der Vegetation einer sowoll durch Menschenhand, als durch Thicre rerwüsteten infra-abietinen Waldregion, wie diese in einzelnen Gebieten des griechischen Reiches, z. B. in Epirus fast unversehrt noch in ihrer ursprünglichen Müchtigkeit besteht, bildet stellenweise eine Fortsetzung der Macchien des Küstengebietes und eine im Inneren Jes Landes an dic Tannenregion sich anlehnende charakteristische Zone. Sie bedeckt die Vorberge am Fusse der Gebirge und diese selbst bis zu einer Seehöhe von 700-1000 $m$ mit ihrem dunkelgrünen Gestrüppe, denselhen von der Entfermung ein cigenthümliches, wolliges Ansehen, möchte man sagen, verleihend. Die sie bildenden Individuen sind krüuppel im wahren Sinne des Wortes, dazu gewrorden durch unvernünftige Abholzung einerseits und durch die zahllosen Schafe und Ziegen andererseits, die Mangels einer passenderen Nahrung, die jungen Triebe, trotz ihrer stechenden Blïtter, abfressen und hiedurch das Höhenwachsthum gründlich beeinträchtigen. Dass dem thatsächlich so sei, beweisen die einzelnen besser entwickelten Stämme und die hier und da selbst zu kräftigen Bäumen ervachsenen Exemplare. Die von der Kermeseiche gebildeten ausgedehnten Dickichte sind stellenweisc cbenfalls fast unpassirbar, sind aber auch häuligg unterbrochen durch weite Strecken Weidelandes, auf dessen steinigem Boden allerdings nur eine meist kürrgliche Vegetation gedeiht. Diese Strecken sind es auch, auf welchen hauptsächlich Feld- und manchmal auch noch WVeinbau betrieben wird; der minder ertragsfähige Theil derselben wird dagegen als W'eide rerwendet, auf welcher hin und wieder Eichen, Accr monspessulamm, Promus pscudoarmoniaca, Ostı_a carpinifolia und Pirms amyggdaliformis oft zu ansehnlichen Bämmen erwachsen und auch Sträucher von .Iminiperus oxycedrus, Liguslumm, Plyyllirea, Colutea, Calycotome, Pistacia Lentiscus, Weissdorn und Rosen gedeihen. Die fliessenden Wïsser, stellenweise sumpfige Niederungen, wie hei Kalavryta erzeugend, ja selbst kleinere Seen bildend, wie den Pheneon-See bei Gura, sind gewöhnlich ron, mitunter zu kleinen Auen sich associirenden Platanen umsäumt.

Die Pflanzenarten, welche in der Kermeseichen-Formation oder auf den von ihr nicht direct occupirten Zwischenstrecken vorkommen, sind äusserst zahlreich, haben jedoch hinsichtlich der Individuenzahl der einzelnen Arten im Verhältnisse zu der der lermeseiche eine secundäre Bedeutung. Sie recrutiren sich in den niederen Lagen aus den Arten der eingangs emrähnten Formationen, in den höheren dagegen aus jenen der Tannenregion. Die wichtigsten derselben sind folgende: Ranmuculus Sprmucrianus, Coratociplatus falcatts, Delphininm ajacis, Papancr Rhocas. Fumaria officinalis, Sisymbrum oricntale und officinale, Berteroa obliqua. Alyssum oricntale und argentem, Hirschfeldia, Clypenla jonthlaspi, Lepidium latifolium. Capsella bursa pasloris, Reseda lulea. Cistus incanns. Helianthcmum salicifolium und vivide. Silcue congesta, italica, viridiflora, radicosa und Reinholdii, Saponaria gracca. Gypsoplhila polygonoides, Tunica illyrica und glumacea, Qucria hispanica, Hyperimm olympicum und empetrifolinm, Malva siluestris, Geranium asplhodcloides und rotmatifolium, Genista Sakellariadis, Ononis subocculta, Trifolinm anghstifolium, scabrum, hirtum, stcllatun. arvense, amrantiacum und campestre, Bonjcania hivsuta, Dorycnium lucrbacenm. Onotrychis cbenoides. Lathyrus grandiflorns und pratcnsis, Orobus niger, l'icia striata, Potcrium muricatum. Tclephium oricutale, Selum laconicum und mbens. Orlaya platycaypos, Turgenia latifolia. Fermlago monticola und nodusa. Bonamia graeca, Malabaila anrea, Oenanthe pimpinelloides, Pim-

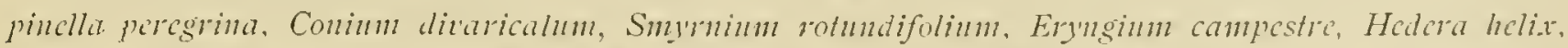

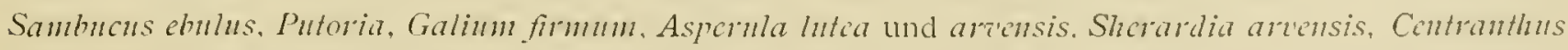

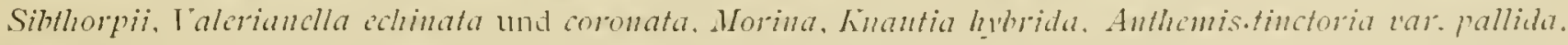
Achillea ligustica, . Teranthemmm inapertum. Onopordon illyricum. Chamatence stellata und Afra. Sillwum 
marianum, Cirsinm acamu. Carduns plenocoplualus, hentroplyylnm lanatum, Centaurca cyanus, calcitrapa

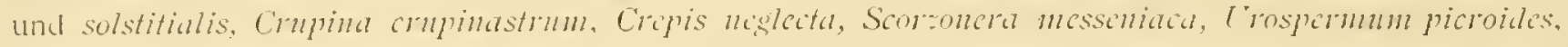
Helminthia cchioides, Leoutulon gracens. Hypochacris iretensis, Scolymus. Specularia hybrida, Contolvulus cantabrica und temissimus, Lycopsis variegala, Unosma frutescins, Tirtascum Sartorit. Scrofularia

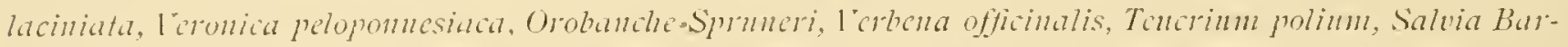

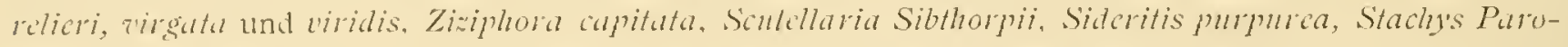
linii, Phlomis fonticosa, Ballota actabnhosa, Micromeria juliana. Thymus atticus, Chanbarli und capitatus, P'rimula acaulis, Plantago lamcolata, Rumetr graecus und pulaher. Osyris, Iris Sintenisii, Ruscus aculeatus. Dactylis hispanica. Festucu Heldreichii und valcsiaca. Cymosmrus echinatus, Bromms sterilis und tectormm.

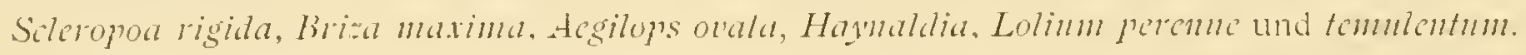

Die Formation der sumpfigen Niederungen setzt sich zusammen aus: Nasturtinm officinale, Althaca officinalis, Trifolinn resupinatum und nigrescens, Potentilla reptans, Epilohium hirsulum, Galinm clonGatum, Cirsium siculum. Solanmm anlamara. Iironica Anagallis, Lysinachia atropurpurea, Plantago

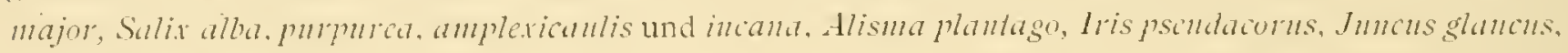
Sparganium ramosum. Cuperus longus, Alopoums utriculatus, Plragmites communis. Giljceria plicata. Equisctum palustro.

Wie die unteren Formationen der eigentlichen Mediterranregion im engeren Sinne ron der Küste her almälig an die zweite Region, deren charakteristischen Repräsentanten die Kermeseichenformation darstellt, sich angliedern, ebenso geht auch diese nur successive in die dritte Region, in jene der Tanne über. Erst einzeln und oft verkrüpelt, bald aber zu mehr minder dichten, unvermischten Beständen sich anordnend, bilden diese Bäume eine ausgedehnte Formation, welche im mächtigen Gürtel die griechischen Gebirge umgibt. Sie ist bezüglich ihrer unteren und oberen Höhengrenze mannigfachen Schwankungen unterworfen, da sie zungenförmig in die an sie grenzenden Regionen hineingreift. Die Exposition übt jedenfalls den bedeutendsten Einlluss aul ihre Grenzen aus, doch ist hierüber zur Zeit nichts Endgiltiges zu sagen, da nur eine geringe Zahl von diesbezüglichen Beobachtungen vorliegen. Auch die nachfolgend verzeichneten Resultate meiner Messungen sind zu lückenhaft, um aus ihnen Schlüsse ziehen zu können. Diese ergaben als:

$$
\text { Exposition Meter }
$$

Untere Cirenze der Tamnenregion:

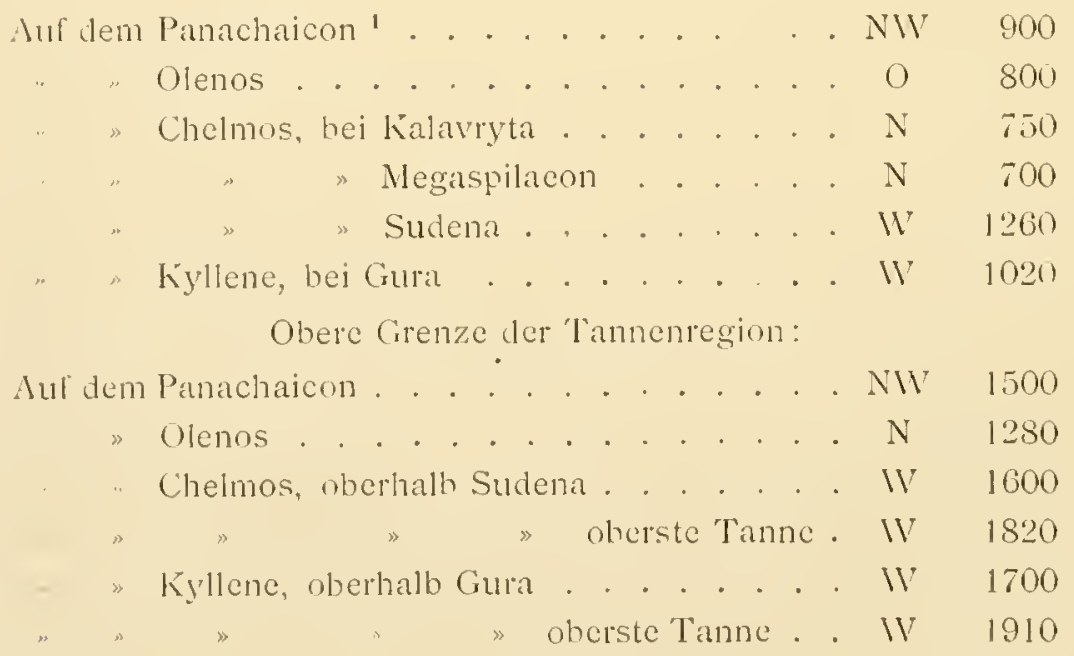

In die untere Tannenregion geht noch hier und dort Pirus ambsiduliformis und Querens mulhescus hinein in einzelnen Exemplaren und entlang der Cehirgshäche die Platane, deren ohere Grenze auf dem Chelnos oherhalh Planideri bei $1020 \mathrm{~m}$ festgestellt wurde. An den nördlichen Abhängen des Olenos, oher- 
halb von Hagios l'lasios, tritt bei $1300 \mathrm{~m}$, der oheren Tannengren\%e sich anschliessend, ein sehütterer, atus krätigen alten Bäumen gebildeter Bestand von Immip'rms focticlissimu aul.

Das Unterholz der Tannenregion ist spärlich und einfömig und wird rorwiegend aus Juminerns nul¿cdrus, viel seltener aus cinzelnen Weissdornsträuchern gebildet; dagegen ist der Niederwuchs sehr artenreich. Es wurden in diesem nachstehende Arten beobachtet: Ranmanlus Sprmmirimms, Arabis mmralis,

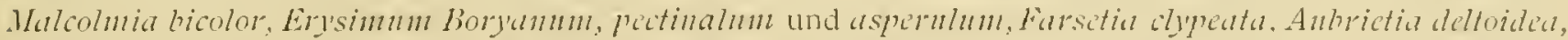

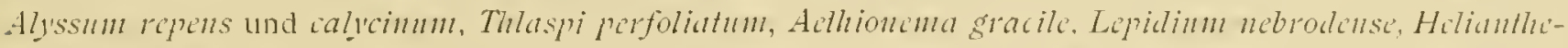

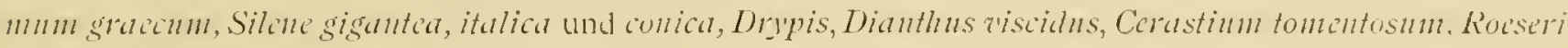

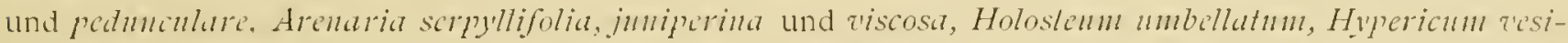

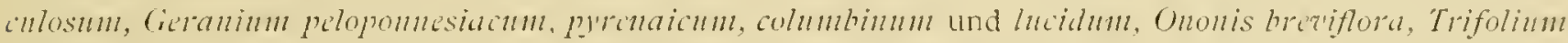
l'iguantii und speciosmm. Coronilla imbroides, Astragalus Chambardi und creticus. Orobus hirsmtus und

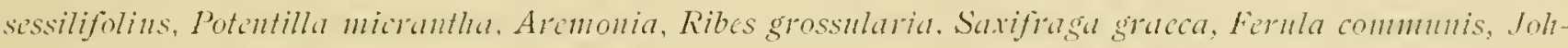

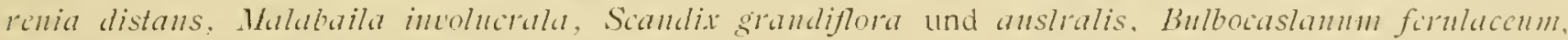

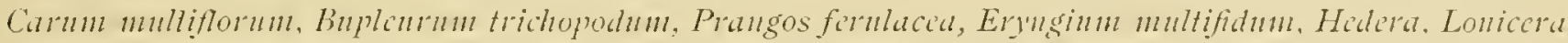

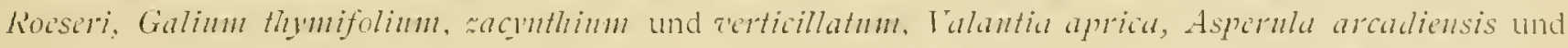

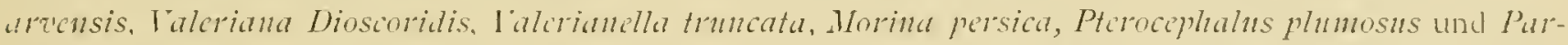
nassi, Doronichm cancasicmm. Senceio thapsoides und rmpeslris, Anthomis Brachmanmi und montana. Achillea ligustica. Matricaria Chamomilla, Filago spathnlata. Chamacpanco Afra. Carduns tajegetens,

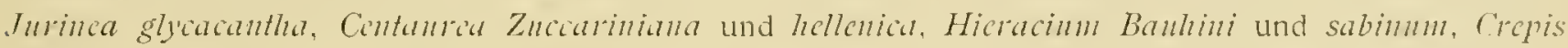

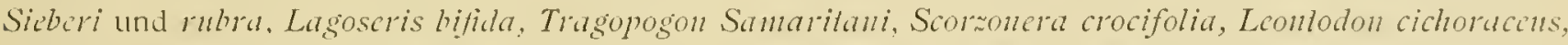
Hypochacris cretensis, Campanula tomentosu und spathnlata, Sinmplytum bulhosmm, Onosma angmstifolium, Alkanna Pulmonaria, Lithospermmm apmbm, Myosotis sitratica und arensis, levbascmm macrourum, Celsia Darnacri, Digitalis fermginea, leronica peloponnesiaca und panommitana, Enfragia latifolia, Tencrinm chamadry's und prostratum, Ajuga oricntalis, Salvia ringans, Stachys critica und gratia. Phlomis finticosa und samia, Mambinm cyllenimm. Clinopodimn. Calamintha snaveolcns und alpina,

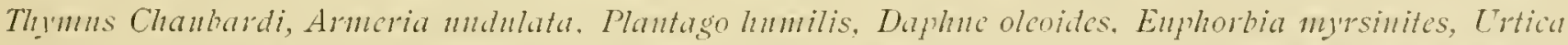
dioica, Ceplabanthera alba, Orchis quadripnnctala. Anacamptis, Llojulia gracca, Ormithogalum fimbriatum und temifolimm, Mascari comosum, Allinm trifoliatum, Luzmla nodosa und Forsteri, Arena filifolia, Aira capillaris, Dactylis, Bromms squarrosns, Poa Timoleontis, Pteris anmilina, Aspleminm trichomanes, Celerach, Aspidium pallidum.

An einzelnen rom Baumwuchse nicht occupirten Stellen bildet in geeigneten Lagen ein Theil dej angeführten Arten eine zusammenhängende Formation mit wiesenartigem Charaliter.

Am Ostabhange des Chelmos, in einer Seehöhe ron 1000--1350 m, wo dieses Gebirge mit jenem des Durduvana mittelst eines Sattels verbunden erscheint, ferner auf den dem Chelmos in Norden rorgelagerten Vorbergen, oberhalb der Ortschaft Zachuli, schiebt sich in die Tannenformation eine Formation von Pinus ligra Arm. ein und bildet daselbst ausgedehnte unvermischte Waldungen. Jie Flora in diesen ist jener der Tannenwälder analog, es wurden darin jedoch einige in der lannenformatimn nicht beobachtete Arten notirt, wie: Clematis vilalla. Ensimmm cuspidatum, Hammatolobinm lotoides, Orobns niger, Rosa haldensis, Galimm crnciala, Bellis perennis. Podanthum limonifolinm, Melittis melissoplyzlnm, Pingnicula hirtiflora, Ostrga, Anthericum Litiago, Carer macrolepis und distans. Melica minfora.

In einer Seehöhe ron 1900 m im Maximum, meist jedoch schon in tieferen Lagen, erreicht die Tannenregion, nachdem ihre Bestände allmälig schütterer geworden waren und oft nur mehr ein einzelnstehender Batum, den Unbilden des Klimas noch Trotz bietend, ihre in früheren Zeiten stattgehabte Ausbreitung kenn. zeichnet, ihre obere Grenze. Nackte Felswände und Steinwüisten, mangels der Grasmatten von der Ferne bar von jeder Vegetation erscheinend, sind der Boden, aus welchem die griechische Hochgebirgsflora ihre kärgliche Nahrung schöpft. Sie ist aber dennoch in hohem Grade mannigfaltig und artenreich, und lässt sich ohne besonderen Zwang in drei, allerdings nicht streng gesonderte Gruppen, eintheilen: in dic Flora der Steinhalden, in die Felsenflora und in jene der Schnecfelder. Die erste ist als die 
reichhaltigste zu bezeichnen. Sie wird stellenweise, insbesondere in groben Gerolle der tieferen lagen. durch einige höhere Pllanzen, wie Clamuepence afra. Carduns hagetchs und Adonis cyllonea charakterisirt und lührt selbst noch vereinzelte Sträucher, wie Prmuls prostrata, Dapllne olvoites und Wachholder als Repräsentanten der Holzgewächse in ihrer Mitte. Die den beiweitem grössten Theil der Hochgebirgsregion ausmachenden, aus zerklüftetem, fast humuslosen Boden bestehenden Strecken, sind jedoch von verstreuten, niedrigen, meist perennirenden Arten bewachsen, welche nur selten zu einem geschlosseneren, an dic Alpenmatten erinnernden Verband, sich associiren. Am häufigsten wurden derlei Strecken mit alpenweideartigem Charakter noch auf dem Panachaicon angetroffen.

Nachfolgende Arten wurden in der eben skizzirten Formation beobachtet: Ranunculus psilostachy's und Sartorianus, Corydalis pamassica und densiflora, Arabis oclurolenca, Erysimmm Boryanum, Draba muralis, Alyssum montanm und calycimm, Thlaspi perfoliatum und graccum, Lepidium nebrodellse, I'iola gracilis und Hercurii, Polygala vulgaris und microcarpa, Geranim subaulescens und macrostylum, Astragalus angustifolins und depressus, Onobrychis laconica var. cacspitosu, Herniaria incana, Scheranthas werticillatus, Scandix gandiflora, Fregera parmassica, Valantia apria, Senecio rupestris, Centaurea cana, Taraxacum laevigatum, Lithospermum incrassatum, IIyosotis cadmea, hispida und refracta, Celsia acanlis, leronica thymifolia, arensis und hederifolia, Laminm nivale, Calamintha alpina, Plantago gracat, Rumex tringnharis, Sternbergia colchiciflora, Fritillaria Guicciardii. Tulipa anstralis, Ormithogalum montammm, nanmm und oligophyllum, Mhscari Heldroichii und commutatum, Carer laevis, Alopecurns Gerardi, Poa parnassica.

Einzelne der angeführten Arten gehen natürlich auch auf die benachbarten Felsen über; in ihren Ritzen und Spalten besitzen aber letztere eine Reihe von Arten, die ihnen fast ausschliesslich eigen sind und die deshalb als besondere Gruppe, die der Felsenpllanzen, betrachtet zu werden verdienen. Zwei von ihnen sind Sträucher: Rlammus Sibthorpiamus und Sorbus gracca; die übrigen zumeist perennirende Kräuter: Ramunculns cadmicus, Arabis cancasica,. Aubrietia deltoidea, Draba affinis und irostra, homiga rupestris, Alyssum oricntale ear. alpinum. Acthionema gracile, Viola chelmea. Alsinc Gerardi, Saxifraga Sartorii z'ar. eryHhranlha, Friderici Angusti, exarala, tridactylites, parnassica und chrososplonifolia, Aspirnla arcadicnsis, lableriana olenaea, Acliblla nmbellata, Globularia stygia, Sestaria coerulans.

An Arten zwar arm, aber oft durch die Nenge der Individuen und durch die lebhaften Farbencontraste ihrer Blüthen ausgezeichnet, ist die Flora der Schneefelder. Sie besteht aus: Animone blanda, Ramuncmlus brevifolins und ficarioides, Crocus Sicheri und Scilla mizulis. An den von diesen Schneeleldern gespeisten Quellen wachsen gewöhnlich Ballis peremis und lironica becabungă.

\section{Itinerarium.}

Jen 30. Nai 1893 landete ich mit den Herren C. Grimus v. Grimburg aus St. Pölten und C. Schwarzenberger aus Wien, in deren Gesellschatt ich auch die weitere peloponnesische Reise machte, in Patras. Wir verblieben in dieser Stadt bis zum 10. Juni und unternahmen täglich Excursionen in deren Umgebung, darunter eine grössere aul den Panachaicon. Am 11. Juni fuhren wir von Patras über Chalandritza, lopesi etc. nach Hagios Vlasios am Fusse des Olenos, welchen Berg wir Jen folgenden Tag ungünstigen Wetters wegen nur bis zur oberen Grenze der Tannenregion erstiegen. Am 13. Juni fuhren wir über Manesi und Saradi nach Kalarryta, wo wir bis zum 19. verblieben, während welcher Zeit wir mehrere Excurse in die Umgebung, darunter eine durch das Voreikos - Thal nach dem Kloster Megaspilaeon unternommen hatten. Am 19. gingen wir nach Sudena und bestiegen den folgenden 'Tag von hier aus den Chelmos. Den 21. verblieben wir in Sudenal, bestiegen den darauffolgenden Tag den Chehnos zum zweitenmale über Planideri, überschritten den Durduvana-Sattel und gelangten, bei Syvistal vorüber, hinab in das AroaniaThal. Wir überschritten dasselbe und bezogen (Uuartier in Gura an Fusse der Ky!lene. Am 24. erfolgte die Besteigung dieses Gebirges. Am 25. ritten wir üher Zachuli nach der Bahnstation Dervenion am Golfe von Corinth und fuhren den nächsten Tag nach Athen. 


\section{Aufzählung der auf dieser Reise beobachteten Arten.}

Es werden hier nur jene Arten angelührt, welehe an den angegebenen Standorten bisher noeh nicht bcobachtet wurden.

\section{RANUNCULACEAE Juss.}

1. Clematis vitalba L. Sp. pl. p. 544 (1753).

113 Föhrenwäldern an den östlichen Abfällen des Chelmos unterhalb des Durdunana-Sattels mit mächtigen $10 \mathrm{~cm}$ im Durchm. dicken Stämmen, selten. Höhe $1000 \mathrm{~m}$.

2. Anemone blanda Schott et Kotsehy in Österr. bot. Wochenbl. 1V, p. 129 (1854).

In der oberen Region des Panachaicon und Chelmos. Höhe 1600-2000 $\mathrm{nll}$.

3. Adonis cyllenea Boiss., Heldr. et Orph. Diagn. Pl. or. Ser. 11, Nr. 5, p. 5 (I856).

Auf den Abfällen der Kyllene oberhalb Gura, selten. Höhe $1500 \mathrm{~m}$.

4. Ranunculus ficarioides Bory et Chaub. in Exp. scient. Mor. 111, 2, p. 156 (1832). - Ficuria Boryi Heldr. Pl. Graec. exs. a. 1844. - F. pcloponnesiaca Nym. Syll. Fl. Europ. p. 179 (1854).

In der oberen Region des Panachaicon, Chelmos und Kyllenc, besonders in der Nähe der Schncefcldcr. Höhe 1800-2000 m.

5. Ranunculus psilostachys Griseb. Spic. Fl. Rum. et Bithy̆n. 1, p. 304 (1843).

In der oberen Region des Panachaicon. Höhe $1500 \mathrm{~m}$.

6. Ranunculus Sprunerianus Boiss. Diagn. Pl. or. Ser. l, Nr. 1, p. 64 (1842).

In einem Saatfelde in der Tannenregion des Panachaicon. Höhe $1000 \mathrm{~m}$.

Var. subglaber. Caule inferne glabro, foliis parce hirsutis.

In der Tannenregion des Panachajcon und Olenos. Höhe 1300-1500 $\mathrm{m}$.

7. Ranunculus cadmicus Boiss. Diagn. Pl. or. Ser. 1, Nr. 1, p. 65 (1842).

Auf feuchten Felsterrassen in der oberen Region der Kyllene oberhalb Gura, sehr selten. Höhe $2000 \mathrm{~m}$.

Die Exemplare stimmen mit den von Pichler auf Karpathos gesammelten, von Boissier in Suppl. Fl. or. p. 7 als Var. parviflorus bezeiehneten überein, nur sind die Früchtchen mit zerstreuten Härchen besetzt. Neu für Europa.

8. Ranunculus brevifolius Ten. Fl. Nap. IV, p. 345 (1811).

An Schneefeldern und im Kalkgerölle der Gipfelregion der Kyflene. Hühe '2300 11 .

9. Ranunculus Sartorianus Boiss, et Heldr. Diagn. Pl. nov. Ser. 11, Nr. 1, p. 8 (1853).

In der oberen Region des Chelmos und del Kyllene. Höhe 1800-2000 $\mathrm{m}$.

10. Ranunculus trachycarpus Fisch. et Mley. Ind. hort. Petrop. p. 46 (1835).

In Gräiben bei Patras und am Fusse des Olenos bei Hagios Vlasios.

11. Ceratocephalus falcatus 1. Sp. pl. p. 556 sub Runnnculo (1753); Pers. Syn. 1, p. 341 (180.5).

Auf Feldern bei Kalavryta. Höhe $700 \mathrm{~m}$.

12. Delphinium Ajacis L. Sp. pl. p. 531 (1753).

In der unteren Region des Panachaicon, Olenos und Chelmos. Höhe 500-700 $\mathrm{m}$.

\section{BERBERIDEAE Vent.}

13. Leontice altaica 1'all. Act petropol. 11, p. 25.) (1779). - L. wlpinu mihi in Sched.

In der oberen Region des Panachaicon an einer einzigen "Beiku vrisi genannten Stelle, hier jedoch nicht selten. Höhe $1700 \mathrm{~m}$.

Wurde nur im Fruchtstadium angetroffen. - lel hielt die Pflanze anfänglich füj cine neue Art und vertheilte sie unter dem Namen L. alpina: wie ich mich jedoch später überzeugte, ist sie wenigstens in 


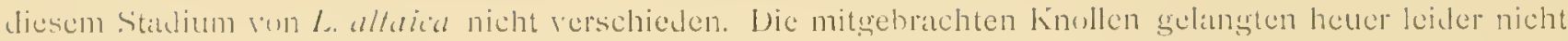
zur lilüthe, und us konnte dihlor eine dieshezügliche endgiltige Identificirung noch nicht rorgenommen werden.

\section{FUMARIACEAE I) (}

1. Corydalis parnassica orph. et Heldr. in Boiss. Diagn. P. or. Ser. H. Ni. 1), J. 0 (1859).

tuf steinigen Triften ler höchsten Erhebungeri des (helmus. Höhe 2200 $\mathrm{m}$.

Lebend durch dic hläulichbereiften Blätter sch auffallend. Sicher cine alusgereichnete und nicht als Subspecies oder Varietit Jer C. car'a zu betrachtende Art.

15. Corydalis densiflora P'res! Delic. Prag I, p. 10 (1822).

In der oberen Region des Panachaicon und Chelmos. Höhe $1600 \quad 18010 \mathrm{~m}$.

Var. teniusecta Boiss. Fl. or. 1, p. 120 (1867).

Nit der Grundform auf dem Chelmos.

IVahrscheinlich gehört hicher C. lemmis Schott Anal. hot. p. 43 (1851).

16. Fumaria officinalis L. Sp. pl. p. $9 \varepsilon 4(1753)$.

In Weingärten bei Hagios Vlasios am Fusse des Olenos. Höhe $6.50 \mathrm{~m}$.

\section{CRUCIFERAE JuSS.}

17. Arabis ochroleuca Boiss. et Heldr. Diagn. Pl. or. Ser. Il, N1. 1, p. 22 (1853).

Iul Triften der oberen Region des Panachaicon, sehr selten. Höhe $1800 \mathrm{~m}$.

18. Arabis muralis Bert. Pl. rar. dec. II, p. 36 (1806).

An Felsen hei Jem Kiloster Megaspilaeon. Höhe $800 \mathrm{~m}$.

14. Nasturtium officinale R. Br. in Ait. Hort. Kew. IV, p. 109 (1789).

In Bächen bei Kalavryta.

20. Malcolmia bicolor Boiss. et Heidr. Diagn. Pl. or. Ser. 1, Nr. 6, p. 10 (1845). M. veluchusis Boiss. I. c. Ser. 11, Nr. 6, p. 10 (18.59).

In der Tannenregion des Chelmos oberhalb Planideri. Höhe $1200 \mathrm{~m}$.

21. Alliaria officinalis D) Syst. I1, p. 489 (1821).

An Bächen des Aroania-Thales nächst dem Orte Syvista. Hühe $800 \mathrm{~m}$.

22.2. Erysimum Boryanum Boiss. et Sprun. Diagn. Pl. or. Ser. 1, Nr. 1, p. 71 (1842).

In der oberen Region des Panachaicon $(1500 \mathrm{~m})$ und in der Tannenregion bei Kalavryta $(800 \mathrm{~m})$.

23. Erysimum pectinatum Bory et Chaub. in Exp. scient. Mor. I11, 2, p. 189 (1832).

In der Tannenregion des Panachaicon. Höhe $1000 \mathrm{~m}$.

2-t, Erysimum asperulum 13oiss. et Heldr. Diagn. PI. or. Ser. 11, Nr. (4, P. 11 (1859).

In der Tannenregion des Panachaicon $(1200 \mathrm{mi}$ ) und am Fusse des Olenos bei Hagios Vlasios (for $\mathrm{m}$ ).

Dic Exemplare stimmen gut überein mit jenen, Welche Heldreich bei Musinitzal am Lorax gesammelt hatte. Sie sind zweijälrig, ihre sichoten vierseitig mit eintachen und Sternharen belicidet, und ich nehme daher auch keinen Anstand, trotzdem einzelne geringfügige Nerlimale der Jiagnose nicht völligg zutrelfen, sic für E. asperulum zu halten.

25. Erysimum cuspidatum M. a. Bicb. F. Taur. Cauc. II, I. 120 sub Chcircuntho (I808); DC. Syst. II, p. $493(1821)$.

In Föhrenwäldern an den östlichen Abfällen des (helmos unterhalb des Durduvana-Sattels, sehr selten. llöhe $1000 \mathrm{~m}$.

26. Sisymbrium orientale L. Amoen. acitd. IV, f. 322 (1750). - S. Colmmule Jacy. Fl. Austr. II: P. $12(1776)$.

. Juf wüsten Plätzen hei Sudena. Höhe $1000 \mathrm{~m} .$. 


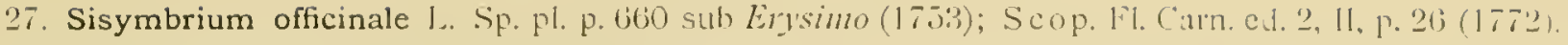
Auf wïsten Plätzen bei Patras und Kilalvyta.

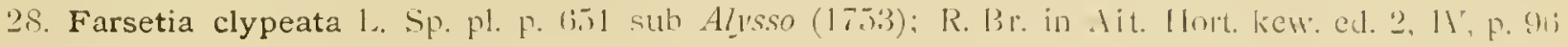
(1812).

In der Tannenregion des Panachaicon. Höhe $1200 \mathrm{~m}$.

29. Aubrietia deltoidea L. Sp. pl. ed. 2, p. 908 sub Alysso (1763); DC . Sy'st. 11, p. 204 (1821).

An Feisen im Thale des Voreikos potamos und des Berges Kastro bei kalaryta. dann in der oheren Region des Chelmos und Panachaicon. Höhe $700-2000 \mathrm{~m}$.

Wie Wettstein in Beitr. Fl. Alban. p. "I nachgewiesen hat, ist auf die Gestalt der Filamente bei der Gattung Aubrictic licin Wesentliches Gewicht zu legen, da dieselhen in verschiedenen Entwicklungs.tadien rertichieden gestaltet erscheinen. Beim Wegfallen der dieshezüglichen Merkmale ist aber A. intermediu Heldr. et Oph. kaum weiter halthar und kann ron $A$. difnided nicht mehr unterschieden werden.

30. Berteroa obliqua Sibth. et Sm. Fl. Graec. Prodr. 11, p. 12 sub Allsen (1813); DC. Syst. 11, p. 292 (1821). - R. gracca Boiss et Heldr. Diagn. Pl. or. Ser. 11, Nr. 1, p. 3in (1853).

Auf lialkfelsen im Orte Lopesi.

Unterscheidet sich ron $B$. mutatilis Vent. Choix de pl. jard. de Cels. p. 85, sub Alysso (180:3)= B. moinubens Portenschl. Enum. pl. Dalm. p. 15) (1824) duich die meist steifere Tracht, häufig röthlich überlaufene Blüthen und durch sternhaarige Schötchen mit aufrechten Stielen: von B. stricta Boiss. et Heldr. Diagn. Pl. or. Ser. II. Nr. 1, p. 35 (1853) durch die elliptischen, nicht verkehrteirundlichen Schötchen.

Zur leichteren Bestimmung der die Balkanhalhinsel bewohnenden brtoroa-Arten diene nachfolgender Schlüssel:

1. Fruchttrauben ziemlich dicht, Schötchen etwas autgeblasen, Samen fast gar nicht oder doch sehr schmal berandet

B illictna. 1.

Fruchttrauhen locker, Schötchen llach. Samen breitberandet, Hautrand 2 mm hreit

2. Kronblätter weiss oder bleichgelhlich. Schötchen verkehrteirundlich bis fast kreisförmig :3

Kronhlätter weiss oder röthlich. Schötchen elliptisch 4

3. Kronblätter weiss, Schötchenstiele aufrecht, länger aJs das Schötchen, letzteres klein, $4-6 \mathrm{~mm}$ lang

B. stricka B. et $\mathrm{H}$.

Kronblätter bleichgelblich, Schötchenstiele auliecht abstehend, sn lang als das Schötchen, letzteres gross, $7-9 m m$ lang

B. orbictlata $\mathrm{DC}$.

4. Kronblätter weiss oder häufig röthlich überlaufen, Schötchen sternharig, ihr Stiel aufrecht

Kronblätter weiss, Schötchen kahl, ihr Stiel aufrecht-abstehend

B. obliqual S. et S.

Dic hicr erörterten fünf naheserwandten Arten haben folgende Verbreitung:

B. inuma. Croatien, Bosnien. Montenegro, Serbien, Walachei, Bulgarien, nördliches Macednnien.

B. Mutabilis. Dalmatien, Hercegovina, Montenegro, nordwestliches Griechenland (Corfu, Actolien, Eury"tanien und nordwestliches Thessalien). Zweifellos in Albanien noch aufzufinden.

B. obliqua. Albanien, Epirus, Aetolien, Peloponnes.

B. stricla. Südöstliches Macedonien (Berg liorthiati) und Thessalien (bei Katerina am Fusse des Olymps und am Pelion).

B. orbiculalu. Südöstliches Nacedonien (bei Kulala, Habinsel Hagion Oros und Berg Kinthiati).

31. Draba affinis Host. Fl. Austr. Il, F. 238 (1831).

In der oheren Region des Chelmos und der Kyllene, selten. Höhe 2000-2300 $\mathrm{m}$.

32. Draba erostra Spec. nov. - Sectio Aizopsis D C. Syst. veg. II. p. 332.

Dense caespitosa, laete rirens; foliis coriaceis, nitidis, linearibus, acutiusculis, pectinato-ciliatis: scapis elongatis, erectis, glabris: lloribus corymbosis, ochroleucis : petalis calycem superantihus; staninibus calycem 
parum excedentibus; siliculis in racemum ovatum vel oblongum dispositis, cum pedicello glabro aequilongo, vel dupio longiore patulis, oblongo-ellipticis, setis simplicibus dense setulosis, stylo subnullo apiculatis.

Maasse: Schaft $5-10 \mathrm{~cm}$ hoch; Schötchen $8-9 \mathrm{~mm}$ lang, $3 \mathrm{~mm}$ breit.

Auf Kalkfelsen des Chelmos oberhalb Sudena. Höhe $2000 \mathrm{~m}$.

Die ebenbeschricbene Art gehört in jene Gruppe der Section Aizopsis, welche durch das Merkmal cines sehr kurzen Griffels charakterisirt ist, und sie ist daher in erster Linie mit I).cretica, compacta und Lacaitac zu vergleichen. D. cretica Boiss. et Heldr. unterscheidet sich von ihr durch den sehr kurzen, beharten Schaft, sehr kurze Traube, längere Staubgefässe und sternharige Schötchen; D. compacta Schott chenfalls durch den sehr kurzen Schaft, durch die compacte Traubc, lüngere Staubgefüsse (so lang als die Blumenkrone), kleinere, spärlich behaarte Schötchen und einen längeren Criffel; D. Lacuitac Boiss. Fl. or. suppl. p. 53 endlich durch ganz kahle Schötchen. Letztere Art, von welcher mir ein im Blüthenstadium hefindliches Originalexemplar vorliegt, wurde erst in neuerer Zeit von L ac a it a, ehenfalls auf dem Chelmos. aufgefunden. Sie ist jedenfalls diejenige, mit welcher D. crostra zunïchst verwandt ist. Ja, es muss sogar dic Mïglichkeit zugegeben werden, dass beide nur Varietïten ein und lerselben Art seien, welche Auffassung durch die bekannte Neigung der Draben, in kahl- und behaartfüchtigen Formen aufzutreten, bekräftigt wird. Der Umstand des Vorkommens auf ein und demselben Gebirge spräche auch hiefür. Da jedoch, abgesehen von der Schötchenbekleidung, einige allerdings mehr nebensächliche Merkmale mit der Diagnose Boissier's nicht völiig im Eirlilange stehen, möchte ich vorderhand beide nicht vereinen. Um dies thun zu können, muss jedenfalls cin reicheres und instructiveres Material von D. Lacaitac vorhanden sein.

33. Draba muralis L. Sp. pl. p. 643 (1753).

In der Tamnenregion des Olenos. Auch am Panachaicon, daselbst schon von Heldreich gesammelt.

24. Koniga rupestris Ten. Fl. Nap. Prodr. 1, p. 37 sub All'sso (1811); Nym. Syll. Fl. Europ. p. 200 $(1854-1855)$.

Auf Felsen der obcren Region der Kỵllene oberhalb Gura, höchst selten. Höhe $2000 \mathrm{~m}$.

3i. Alyssum orientale Ard. Spec. alt. p. 32 (1763).

Auf Felsen bei Kalanistra, Lopezi und Megaspilaeon. Höhe $400-700 \mathrm{~m}$.

Var. alpinum.

Dense caespitosum, culibus pumilis, $3-8 \mathrm{~cm}$ altis, simplicibus; foliis integris subintegrisve; siliculis ovalibus.

In der oberen Region der Kyllene oberhalb Gura. Höhe 2000 m.

Die hervorgehohenen Merkmale bedingen eine sehr auffäligc, von der Grundform gänzlich verschiedene Tracht.

36. Alyssum repens Baumg. Enum. Trans. II, p. 237 (1816).

In der Tamnenrcgion des Olenos. Höhe $1300 \mathrm{~m}$.

37. Alyssum montanum L. Sp. pl. p. 650 (1753).

In der oberon Region des Panachaicon. Höhe $1700 \mathrm{~m}$.

38. Alyssum minutum Schlecht. in DC. Syst. Il, p. 316 (1821).

1 1n der oberen Region des Panachaicon, selten. Höhe $1800 \mathrm{~m}$.

39. Alyssum argenteum Vitm. Summ. IV, P. 430 (1790).

Bei Kalavyta. Höhe $700 \mathrm{~m}$.

40. Alyssum calycinum 1. Sp. pl. p. 908 (1753).

In der Tannenregion des Chelmos oberhalb Sudena. Höhe 1200-1500 $\mathrm{m}$.

Die hier vorgefundenen Exemplare sind ausnahmslos zwergig, ilure Stengel nur 1 -ícm hoch, die liruchttrauben nicht verlängert, die kimblätter ausgerandet bis zweispaltig. In den Exsiecaten wurden sie als var. l'mmilmm ausgegehen. 
41. Thlaspi perfoliatum I. Sp. pl. p. 640 (1753).

1n der Tannen- und oberen Region des Panachaicon und der Kyllene; steigt bis "2000 $\mathbf{m}$ hinatul.

12. Thlaspi graecum lord. Obs. fasc. III, p. 30 (1846).

In der obcren Region des Panachaicon und Chelmos. Höhe $1800-2200 \mathrm{~m}$.

43. Aethionema gracile DC. Syst. II, P. 559 (1821).

In der Tamnen- und oberen Region des Panachaicon und Chelmos. Höhe $1200-2000 m$.

44. Lepidium latifolium I. Sp. pl. p. 644 (1753).

Bci Kallavryta. Höle $700 \mathrm{~m}$.

45. Lepidium nebrodense Guss. Fl. Sic. Syn. II, p. 15+(18t1). - L. microsiylmm Boiss. cet Heldr. Diagr. Pl. or. Scr. JI, Nr. 6, p. 21 (1859).

In der Tannen- und oberen Region des Panachaicon und Olenos. Höhe $1000-1800 \mathrm{~m}$

L. microslylum soll durch den kurzen, aus der Ausrandung des Schötchens kaum hervorragenden Griffel von L. nchrodense verschieden sein. Nach meinen Untersuchungen ist jedoch die Griffellänge bei beiden vermeintlichen Arten variabel, und insbesondere weisen of Exempiare von L. nebrolense, wie sje 2. B. Lojacono in P1. Sic. rar. vom Madonie-Gebirge vertheilte, ehenso kurze Griffel auf, als die griechischen des $L$ microsllmm. Letzteres ist daher, meines Erachtens, als Art nicht aufrecht zu erhaltcn.

\section{CISTINEAE Juss.}

46. Helianthemum salicifolium L. Sp. pl. p. $7+2$ sub Cislo (1762); P'ers. Syn. II, p. 78 (1807). In der unteren Region des Panachaicon und Olenos. Höhe $500-700 \mathrm{~m}$.

47. Helianthemum graecum Boiss. Diagn. Pl. or. Ser. II, Nr. 1. p. 52 (1853).

In der 'Tannenregion des Panachaicon. Höhe $1200 \mathrm{~m}$

48. Helianthemum viride Ten. Fl. Nap. I, p. 299 (I811).

Im Thale des Voreikos bei Megaspilaeon. Höhe $700 \mathrm{~m}$.

\section{VIOLARIEAE DC.}

49. Viola chelmea Boiss. et Heldr. Diagn. Pl. or. Ser. Il, Nr. 1, p. 54 (1853).

In Felsritzen des Chelmos oberhaib Sudena, schi selten. Höhe $1800 \mathrm{~m}$.

50. Viola gracilis Sibth. et Sm. Fl. Graec. Prodr. J, p. 140 (1806).

Var. brevicalcarata Boiss. Fl. or. 1, p. 463 (1867).

11 der oberen Region des Chelmos. Höhe $2000 \mathrm{~m}$.

Identisch mit der Pflanze der Kyllene, wo ich sie ebenfalls beobachtete.

51. Viola Mercurii Orph. Fl. graec. exs. Nu. 401 (1856). - Sectio Melamimm DC. Prodr. 1, p.31)1.

Annua, gracilis, $2-7 \mathrm{~cm}$ alta, indumento papilloso brevi sparsim obsita vel glabrescens, simplex rel basi ramosa; foliis petiolo eis aequilongo vel paulo longiore suffultis, inferioribus ovato-rotundatis, superioribus oblongo-spathulatis, omnibus obtusis repandis subintegrisve; stipulis plerumque tripartitis, segmento medio folio simili, segmentis lateralibus linearibus; pedunculis elongatis; sepalis lanceolatis, integris, appendicibus acutis: petalis calyce duplo longioribus, obovatis, luteis, calcare recto ohtuso, appendices calycis acquante; capsula ovoideo-subglobosa, seminibus fuscis nitidis.

Wurde von Orphanides auf der Kyllene oberhalb Lambani im Jahre 1854 entdeckt. Jch fand sie im vorigen Sommer ebenfalls auf der Kyllene oberhalb Gura, wo sie in einer Seehöhe ron 1500 m im lialkschutte zerstreut vorkommt.

Ich habe hier die Diagnose dieser von Orphanides vor to Jahren in schedis aufgestellten Art gegeben, da von derselben bisher nirgends eine Beschreibung existirt. Wenn auch die der höchst polymorphen Gruppe der 1 . tricolor L. im weiteren Sinne angehörigen Formen oft nur recht künstlich auseinander gehalten werden können, da die meisten durch eine Reihe von Zwischenformen mit einander verbunden sind, so 
finden sich unter ihnen doch wieder eine Anzahl. welche nicht nur habituell, sondern auch durch eine Summe constanter Mlerkmale von den übrigen sich derart als verschieden erweisen, dass sie als gut charakterisirte selbständige Arten betrachtet zu werden verdiencn. Zu letzteren gehört auch die eben beschriebene V. Mercurii. Dieselbe wurde von Boissier in Fl. or. J, p. 466 als var. $\gamma$ Demetria der V. tricolor L. aufgezählt. Boissier erwähnt zwar den von Orphanides aufgestellten Namen daselbst gar nicht; dic von ihm citirte Nummer der Orphanide s'schen Collection jedoch zeigt, dass er thatsächlich diese unter seiner var. $\gamma$ verstanden wissen wollte.

Nit der spanischen V. Demetria Prol. in Bo iss. Voy. p. 73 ist aber meines Dafürhaltens die griechische Pflanze nicht $z$ identificiren, wie nachstehende Tabelle zeigt:

\section{Demetria Prol.}

Blätter gekerbt.

Kelchzipfelanhängsel stumpflich, meist ausgerandet. Blüthen blassgelb.

Sporn gekrümmt, länger als die Anhängsel.

\section{Mercurii Orph.}

Blätter randschweifig oder fast ganzrandig. Kelchzipelanhängsel spitz. Blüthen sattgelb mit violettem Sporne. Sporn gerade, so lang als die Anhängsel.

Mit $V$. Mercurii nahe verwandt ist eine zweite, die griechischen Berge bewohnende Art, nänlich $V$. hymettia Boiss. et Heldr. Diagn. Pl. or. Ser. II, Nr. 1, F. 57 (1853), welche übrigens ausser den von Boissier aufgezählten Standorten am Taygetos und Hymettus auch noch am Parnes und Pentelicon in Attica vorkommt. Diese unterscheidet sich aber von jener hinlänglich durch die gekerbten Blätter, die vielspaltigen, mit fast borstlichen seitlichen Segmenten versehenen Nebenblätter, die stumpfen, ctwas ausgerandeten Kelchzipfelanhängsel und durch die grösseren violettgescheckten Blüthen.

\section{POLYGALEAE Juss.}

52. Polygala vulgaris L. Sp. pl. p. 762 (1753).

In der oberen Region des Panachaicon. Höhe $1800 \mathrm{~m}$.

53. Polygala microcarpa Gaud. Fl. Helv. JV, F. 445 (1829). - P. alpestris Reich cn b. Fl. Germ. exc. F. 350 (1832). Conf. Kern. Schedae ad Fl. exs. Austro-Hung. II, p. 54 et Ill, p. 64.

In Pölstern von Astragalus anğustifolius auf den Gipfel der Kyllene. Höhe 2350 . Neu für Griechenland.

\section{SILENEAE D)(}

54. Silene gigantea L. Sp. pl. p. 418 (1753). - S. italica var. incana Griseb. Spic. Fl. Rumel. et Bithyn. 1, p. 173 (1843). - S. gigantea var. tiridescens Boiss. Fl. or. I, p. 646 (1867). - S. rhodopea Janka in Természetr. Füz. 11, I, (Sep.) p. 1 (1878). - S. psendonntans Panc. Add. Fl. Serb. p. 116 (1884).

In Tannenwäldern bei Megaspilaeon. Höhe $1200 \mathrm{~m}$.

Unbecinflusst noch von Velenovskýs Publication (Sitzungsb. der k. böhm. Ges. Wiss. mathem.-nat. Classe 1893) kam ich zu demselben Resultate, dass nämlich S. hlodopea und S. psendonutans als Synonyme zu S. gigantea L. gezogen werden müssen.

55. Silene congesta Sibth. et Sm. Fl. Graec. Prodr. 1, p. 300 (1806).

Im Thale des Voreikos potamos bei Kalavryta. Höhe $700 \mathrm{~m}$. - Auch bei Mistra am liusse des Taygetus (leg. Orphanides).

56. Silene italica L. Sp. pl. ed 2, p. 593 sub Cucubalo (1763); Pers. Syn. 1, p. 498 (1805).

Var. rubriflora Otth. in DC. Prodr. J, p. 382.

In der Tannenregion des Olenos. Höhe $1500 \mathrm{~m}$.

57. Silene viridiflora L. Sp. pl. ed II, p. 596 (1762).

Bei Saradi nächst Kalavryta. Höhe $700 \mathrm{~m}$. 
58. Silene radicosa Boiss, et Heldr. Diagn. Pl. or. Ser. 1, Nr. 6, p. 24 (1845).

In der unteren Region des Chelmos bei Sudena. Höhe $1050 \mathrm{~m}$.

59. Silene Reinholdi Heldr. Sert. pl. nov. in Atti Congr. Fir. 187t, p. 238.

Im Gerölle am Voreikos potamos bei Megaspilaeon. Höhe $700 \mathrm{~m}$.

60. Silene conica L. Sp. pl. p. 418 (1753).

In der Tannenregion des Chelmos oberhalb Kalavryta. Höhe $900 \mathrm{~m}$.

61. Drypis spinosa 1. Sp. pl. p. 413 (175:3). - D. spinosa subsp. Limnacama M urb. Beitr. zur Kenntn.

Fl. Südbosn. p. 161 (1891). - D. Limnacana Murb. et Wettst. in Biblioth. bot. Heft 26, p. 28 (1892).

In der unteren Region des Chelmos bei Sudena. Höhe $1000 \mathrm{mt}$.

62. Saponaria graeca Boiss. Fl. or. 1, p. 529 (1867).

Im Sande des Voreikos potamos bei Kalavryta. Höhe $700 \mathrm{rm}$

63. Gypsophila polygonoides Willd. Sp. pl. II, p. 690 sub C̈rcubalo (1799). - G. occllatı Sibth. et

Sm. F1. Graec. Prodr. I, p. 281 (1806).

Auf Kallifelsen bei Planideri in der unteren Region des Chelmos. Höhe $1000 \mathrm{~m}$.

64. Tunica illyrica L. Mant. p. 70 sub Saponaria (1767); Boiss. Fl. or. I, p. 520 (1867).

In der unteren Region der Kyllene bei Gura. Höhe $1000 \mathrm{~m}$.

65. Tunica glumacea Bory et Chaub. in Exped. scient. Mor. Ill, 2, p. 340 sub Diantho (1832); Boiss. Fl. or. 1, p. 517 (1867).

Bei Patras und Kalaviyta.

66. Dianthus viscidus Bory et Chaub. in Exped. scient. Mor. Hl, 2, p. 340 (1806).

Grasplätze in der Tannenregion des Olenos. Höhe $1300 \mathrm{~m}$.

\section{ALSINEAE DC.}

67. Cerastium tomentosum L. Sp. pl. p. 440 (1753).

In der Tannenregion des Panachaicon und der Kyllene. Höhe 1200-1500 $\mathrm{m}$.

68. Cerastium Roeseri Boiss, et Heldr. Diagn. Pl. or. Ser. 11, Nr. 1, p. 93 (1853).

In der Tannen- und oberen Region des Panachaicon, Olenos und Chelmos. Höhe 1000-1800 $\mathrm{m}$.

69. Cerastium pedunculare Bory et Chaub. in Exp. scient. Mor. III, 2, p. 130 (1832).

In der Tannenregion des Panachaicon, selten. Höhe $1200 \mathrm{~m}$.

70. Arenaria serpyllifolia L. Sp. pl. p. 423 (1753).

Var. viscida Lois. Not. p. 68 pro spec. (1810).

In der Tannenregion des Panachaicon. Höhe $1000 \mathrm{~m}$.

71. Alsine juniperina L. Mant. p. 72 sub Arenaria (1767); Fenzl Verbr. Alsin. p. 18 (1833). Arenaria nodosa Bory et Chaub. in Exp. scient. Mor. IlI, 2, p. 125 (1832).

In der Tannenregion des Chelmos bei Sudena. Höhe $1300 \mathrm{~m}$.

72. Alsine viscosa Schreb. Spic. Fl. Lips. p. 30 (1771).

In der Tannenregion des Panachaicon. Höhe $1000 \mathrm{~m}$.

73. Alsine globulosa Lab. Pl. Syr. rar. dec. IV, p. 6, tab. 3, fig. 1 sub Arenaria (1812). - Arenaria fasciculata Sibth. et Sm. Fl. Graec. Prodr. 1, p. 336 (1806), non Gouan. - Alsine Smithii Fenzl Verbr. Alsin. p. 57 (1833).

Im Bachkiese bei Patras, in der unteren Region des Panachaicon und in Voreikos-Thale bei Kalavryta. Höhe $30-700 \mathrm{~m}$.

74. Alsine Gerardi Willd. Sp. pl. II, p. 729 sub Arenaria (1799); Wahlent. Fl. Carp. p. 132 (1814). In der oberen Region des Chelmos. Höhe $2000 \mathrm{~m}$. 
75. Queria hispanica L. Sp. pl. p. 90 (1753).

In der unteren Region des Panachaicon und Chelmos. Höhe $1000 \mathrm{~m}$.

\section{LINEAE DC.}

76. Linum pubescens Russell Nat. hist. of Aleppo II, p. 268 (1794).

Lehmberge und W'eingartenränder bei Patras. Höhe $50 \mathrm{~m}$.

77. Linum spicatum Pers. Syn. 1, p. 336 sub var. \% L. stricli L. (180.5).

Weingartenränder bei Patras.

XI. MALVACEAE R. Br.

78. Althaea pallida W. et K. Pl. rar. Hung. 1, t. $47(1800)$.

An Wegen bei Patras und Chalandritza.

79. Malva cretica Cav. Diss. II, p. 67 (1786).

Lehmberge bei Patras. Höhe $50 \mathrm{~m}$.

XII. HYPERICINEAE DC:

80. Hypericum olympicum L. Sp. pl. p. 784 (1753).

Im Aroania-Thale bei Syvista. Höhe $800 \mathrm{~m}$.

81. Hypericum crispum L. Nant. p. 106 (1767).

Bei Patras.

82. Hypericum empetrifolium Willd. Sp. pl. 11I, p. 1452 (1800).

In Macchien bei Patras und in Vorcikos-Thale bei Kalavryta. Höhe $100-700 \mathrm{~m}$.

\section{ACERINEAE DC}

83. Acer monspessulanum L. Sp. pl. p. 1056 (1753).

Var: microphyllum Boiss. F1. or. 1, p. 951 (1867).

In đer unteren Region des Olenos bei Hagios Vlasios. Höhe $7.50 \mathrm{~m}$.

\section{GERANIACEAE DC:}

84. Geranium subcaulescens L'Hér. in DC. Prodr. 1, p. 640 (1824).

In der oberen Region des Panachaicon und Chelmos. Höhc 1700-2200 m.

8.5. Geranium tuberosum L. Sp.pl. p. 680 (1753).

Var. macrostylum Boiss. Diagn. Pl. or. Ser. I, Nr. 1, p. 58 pro spec. (1842): Boiss. Fl. or. 1, p. 873 (1867).

In der oberen Region des Panachaicon, Olenos und Chelmos. Höhe $1500-2000 \mathrm{~m}$.

86. Geranium peloponnesiacum Boiss. Diagn. Pl. or. Ser. 11, Nr. 1, F. 110 (1853).

$1 \mathrm{n}$ der Tannenregion des Olenos, selten. Höhe $1500 \mathrm{~m}$.

87. Geranium asphodeloides Burm. Specim. bot. de Geran. p. 228 (1759).

In Qucrcus coccifcra-Gebüschen bei Manesi. Höhe $700 \mathrm{~m}$.

88. Geranium pyrenaicum L. Mant. p. 97 (1767).

In der Tannenregion des Olenos. Höhe $1500 \mathrm{~m}$.

89. Geranium columbinum L. Sp. pl. p. 682 (1753).

In der Tannenregion des Panachaicon. Höhe $1000 \mathrm{~m}$.

90. Geranium lucidum 1. Sp. pl. p. 682 (1753).

In der 'Pannenregion des Chelmos und Olenns. Höhc $1200 \mathrm{~m}$. 
XY. RHAMNEAE R. Br.

91. Rhamnus Sibthorpianus Roem. et SchuIt. Syst. VI, p. 286 (1817).

An Felsen der oberen Region des Chelmos. Höhe $2000 \mathrm{~m}$.

\section{XYI. PAPILIONACEAE L.}

92. Genista Sakellariadis Boiss. ct Orph. Diagn. Pl. or. Ser. 11, Nr. 6, p. 42 (18.59).

Unter Büschen ron Qucreus coccifera aul dem Panachajcon. Höhe $1000 \mathrm{~m}$.

93. Ononis reclinata L. Sp. pl. ed. 2, p. 1011 (1763).

Var. mollis Savi. in Mem. Soc. ital. IX, p. 351 pro spec. (1802). - O. Cherleri. 1)esf. Fi. Atl. 11, p. 148 (1800) an L. - O. reclinata var. minor Moris Fl. Sard. I, p. 421 (1837).

Bei Patras.

9. Ononis breviflora DC. Prodr. 1I, p. 160 (1825).

In der Tannenregion bei Megaspilacoı. Höhe $800 \mathrm{~m}$.

9.). Ononis pubescens L. Mant. p. 267 (1767).

In Macchien bei Patras.

96. Ononis subocculta Vill. Prosp. hist. pl. Dauph. F. 4I (1779). - O. Colnmunac All. Fi. Ped. I, F. $318(1785)$.

in der unteren Region bei L.opesi.

97. Melilotus neapolitana Ten. F1. Nap. Prodr. Suppl. 1, p. 56.

In Macchien bei Patras.

98. Trifolium Pignantii Fauché et Chaub. in Exp. sciont. Mlor. 111, p. 219 (1832).

In der Tannenregion bei Megaspilacon. Höhe $800 \mathrm{~m}$.

99. Trifolium Cherleri L. Amoen. acad. IV, p. 286 (1759).

Bei Eglikada nächst Patras.

100. Trifolium hirtum All. Auct. Ped. p. 20 (1789).

Bei Hagios Vlasios am Fusse des Olenos. Höhe $700 \mathrm{~m}$.

101. Trifolium stellatum L. Sp. pl. p. 769 (1753).

Bei Patras, Kalavryta und Sudena. Höhe $20-1000 \mathrm{~m}$.

102. Trifolium arvense L. Sp. pl. p. 769 (1753).

Bei Hagios Vlasios am Fusse des Olenos. Höhe $700 \mathrm{~m}$.

103. Trifolium pallidum IV. ct K. Pl. rar. Hung. I, p. 35 (1802).

In Macchien bei Patras.

104. Trifolium physodes Stev. in M. a Bieb. Fl. Taur. Cauc. II, p. 217 (1808). - T. Cupani Ten.

Pl. rar. sic. pug. p. 16 (1817). - T. anomalum Bory et Chaub. in Exp. scient. Mor. III, 2. p. 221 (1832). T. ovalifolium Bory et Chaub. Fl. Pelop. p. 51 (1838).

Auf Lehmbergen bei Patras.

105. Trifolium speciosum Willd. Sp. pl. 111, p. 1382 (1800).

In der Tannenregion des Panachaicon und des Chelmos oberhalb Planideri. Höhe 1100-1400 m.

106. Trifolium aurantiacum Boiss, et Sprun. Diagn. PI. or. Ser. I, Nr. 2, p. 33 (1843).

In Gebüschen von Quercus coccifora auf dem Panachaicon, selten. Höhe $1000 \mathrm{~m}$.

107. Dorycnium herbaceum Vill. Hist. pl. Dauph. III, p. 417 (1789).

In der unteren Region des Chelmos und Olenos. Höhe 300-800 m.

108. Hammatolobium lotoides Fenz.1 Pug. pl. nor. Syriae et Tauri occ. primus p. 3 (1842); I11. et descript. pl. nov. Syriae et Tauri occ. p.1 (1843). H. graccum Heldr. in Boiss. Fl. or. suppl. p. 171 (1888). 
In Föhrenwäldern am Durdurana-Sattel des Chelmos zwischen Planideri und dem Aroania-Thale unweit der Quelle Turcorrysi zerstreut an mehreren Stellen. Höhe $1400 \mathrm{~m}$.

Eine äusserst seltene, für Europa bisher nur vom Taygetus bekannte Art, wo sie Pich ler im Jahre 1876 sammelte und in seinen Exsiccaten als H. graccum Heldr. vertheilte. Unter diesem Namen wurde sie auch von Boissier a. a. O. als eine neue, von $H$. lotoides verschiedene Art beschrieben.

Nach letzterem Autor soll sie sich von H. lotoides durch dreizählige kleinere Blätter, einblüthige Pedunkeln, einfache, meist dreizählige Deckblätter und kleinere, purpurngefärbte Blüthen unterscheiden.

Diese Merkmale treffen allerdings an den mir vorliegenden Exemplaren vom Taygetus zu, sind aber, wie ich mich an zahlreichen, von mir am Chelmos gesammelten Exemplaren überzeugen konnte, nicht durchgreifend. Ich fand hier nämlich Individuen, besonders an steinigen, wenig Humus enthaltenden Stellen, welche vollständig der. Tayget-Pflanze und deren Beschreibung entsprachen, dann aber auch im fetten Erdreiche wieder solche, die sowohl mit der Diagnose, als auch mit den kleinasiatischen Originalexemplaren des $H$. lotoides übereinstimmten. Diese beiden habituell recht verschiedenen Endglieder waren jedoch verschiedenartig durch \%wischenformen verbunden, ja ich fand sogar zuweilen die Merkmale beider, wie z. B. drei- und fünfzählige Blätter, ein-bis vierblüthige Pedunkeln, ein- bis dreizählige Deckblätter an ein und demselben Individuum vor. Auch die Farbe der Corolle variirte, bald war selbe durchaus goldgelb, bald wieder die Fahne intensiv purpurn gefärbt; ebenso war die Grösse der letzteren und auch jene der Blättchen, wie auch endlich die Behaarung aller Theile erheblichen Schwankungen unterworfen.

Mit Berücksichtigung dieser. Umstände kann daher $H$. graecum nicht als selbständige Art betrachtet werden, sondern ist als magere Form des $H$. lotoides aufzufassen.

109. Hippocrepis glauca Ten. Fl. Nap. Il, p. 165 (1811).

Mit voriger Art in Föhrenwäldern des Chelmos.

110. Astragalus angustifolius Lam. Dict. Enc. meth. I, p. 321 (1789).

In der oberen Region des Panachaicon, Chelmos und der Kyllene. Höhe $1800-2300 \mathrm{~m}$.

111. Astragalus creticus Lam. Dict. Enc. meth. I, p. 321 (1789).

In der Tannenregion des Chelmos oberhalb Kalavryta. Höhe $1000 \mathrm{~m}$.

12. Astragalus depressus L. Sp. pl. p. 1073 (1763).

In der oberen Region des Panachaicon und Chelmos. Höhe $1800-2200 \mathrm{~m}$.

113. Onobrychis laconica Orph. in Boiss. F1. or. 11, p. 530 (1872).

Var. caespitosa.

Eine kleine rasige Varietät mit sehr verkürztem Stengel in Astragalus-Polstern auf dem Gipfel der Kyllene. Höhe $2375 \mathrm{~m}$.

Boissier erwähnt sie schon a. a. $O$.

14. Onobrychis ebenoides Boiss. et Sprun. Diagn. P1. or. Ser. 1, Nr. 2, p. 97 (IS43).

Bei Kalavryta und Megaspilaeon. Höhe $800 \mathrm{~m}$.

115. Onobrychis aequidentata Sibth. et Sm. Fl. Graec. Prodr. 11, p. S4 sub Hedysaro (1813); Urv. Enum. p. 90 (1822).

Lehmhügel bei Patras.

116. Lathyrus grandiflorus Sibth. et Sm. Fl. Graec. Prodr. 11, p. 67 (1813).

An Rainen bei Gura. Höhe $900 \mathrm{~m}$.

117. Lathyrus pratensis l. Sp. pl. p. 733 (1753).

An Bachrändern im Aroania-Thale bei Syvista. Höhe $650 \mathrm{~m}$.

118. Orobus hirsutus L. Sp.pl. p. 728 (1753).

In der 'Tannenregion des Panachaicon unu Chelmos, nicht gemein.

119. Orobus niger L. Sp. pl. p. 729 (1753).

An Bachrändern im Aromial-Thale hei Syrvista, sehr selten. Höhe $6.00 \mathrm{~m}$. 
120. Orobus sessilifolius Sibth. et Sm. Fl. (irace. Prodr. 11, p. (it (1813).

In Aer Tannenegion des Panachácon. Höhe $1200 \mathrm{~m}$.

121. Vicia salaminia Heldr. et Sart. in Boiss. Dingn. 1'. or. Ser. 11, Nr. 2, p. 39 (1856).

In Gebüschen bei Patras gemcin.

122. Vicia striata M. a 13. 1il. Taur. Cauc. 11, p. 162 (1808). - 17. pmpurascens DC. Hort. Monspel p. $155(1813)$.

An IVegen bei Saradi.

\section{ROSACEAE Juss.}

123. Prunus pseudoarmeniaca Heldr. et Sart. in Boiss. Diagn. Pl. or. Ser II, Nr. 5, p. 96 (1856). In der unteren Region des Chelmos bei Sudena und Planideri. Höhe $1000 \mathrm{~m}$.

124. Prunus prostrata Lab. Ic. pl. Syr. rar. dec. J, p. 15 (1791).

In der oberen Region des Chelmos. Höhe $2000 m$.

125. Crataegus pycnoloba Boiss. et Heldr. Diagn. Pl. or. Ser. 11, Nr. 2, p. 46 (1856).

In Föhrenwäldern oberhalb des Dorfes Zachuli. Höhe $800 m$.

126. Crataegus Heldreichii Boiss. Diagn. Pl. or. Ser. II, Nr. 2, p. 47 (1856).

Bei Kalavryta. Höhe $700 \mathrm{~m}$.

127. Potentilla micrantha Ram. in DC. Fl. Tr. IV, p. 468 (1805).

In der Tannenregion des Olenos. Höhe $1200 \mathrm{~m}$.

128. Rosa sempervirens L. Sp. pl. p. 492 (1753).

In Macchien bei Patras.

129. Rosa baldensis Kern. in Deségl. Cat. rais. in Bull. soc. roy. bot. Belg. XV, p. 217 (J876).

In Föhrenwäldern bei Syvista unterhalb des Durduvana-Satteis. Höhe 700 m.

130. Rosa arcadiensis. Species nova. - (Sectio Rubiginosae).

Frutex elevatus, ramis subflexuosis, ramulis fertilibus brevibus, aculeis hamato-falcatis, robustis, ad basin dilatatis, in ramulis fertilibus gracilibus aduncis; stipulis lanceolatis, auriculis acutis divergentibus, margine vel inferioribus etiam in lamina glandulosis; foliolis quinis, rarius septenis, parvis (10-18 $m m$ longis, $8-16 m m$ latis) late-ellipticis vel suborbicularibus, subtus ad costam primariam pilosiusculis, ubique glandulis odorantibus et rufescentibus crebre adspersis, supra glabris, in margine composite glandulososcratis, dentibus serraturae acutis, profunde in laminam foliorum incisis; bracteis saepe foliaceis, in lamina cglandulosis; pedunculis brevibus, setis glanduliferis sparsis armatis; receptaculis ellipsoideis glabris, sepalis duobus integris, tribus pinnatifidis, post anthesin reflexis, dorso glandulis stipitatis sessilibusque sparsim praeditis, margine glanduloso-ciliatis tomentosisve; petalis mediocribus, lacteis; disco subconico, stylis villosis; receptaculis fructiferis ellipsoideis.

Die ebenbeschriebene Rose scheint im Chelmos-Gebiete verbreitet zu sein; ich fand sie an mehreren Stellen in der unteren Region dieses Gebirges in einer Seehöhe von $700-1000$ m. so bei Megaspilaeon, Kalavryta und Sudena, wo sie an geschützten Orten ziemlich ansehnliche Sträucher bildet, an von Schafen und Ziegen besuchten Stellen jedoch krüppelhaft wird und in der Tracht dann sehr an $R$. glulinosa erinnert. Ich beschrieb sie als neue Art, weil ich sie mit keiner der mir bekannten Rosenformen identificiren konnte und weil sie auch dem ausgezeichneten Rhodologen Crépin unbekannt war. Sie erinnert in ihrer Serratur an die Sepiaceen, gehört aber der rundlichen Blättchen wegen zu den Rubiginosen. Durch diese Blättchenform weicht sie auch wesentlich ron der, in vielen Jlerkmalen mit ihr übereinstimmenden $R$. dorica Br. et Hal. ab und nähert sich hiedurch zweifelsohne zur R. sicula Tratt., die jedoch von ihr durch die ungleiche Bestachelung schon genügend sich unterscheidet. Die ihr vielleicht zunächst verwandte Rubiginose, die $R$. lacliflora, ist von ihr durch die dichtdrüsigen Pedunkeln, die am Grunde drüsigen Receptakeln und durch die kahlen oder nur wenig behaarten ( $R$. lactiflora $f$. polyacautha B orb.) Griffel abweichend. 
131. Rosa leucadia Braun in Veris. zool. bot. Ges. Wien XXYY, T. 126 (1885).

Wegränder bei Lopesi. Höhe $300 \mathrm{~m}$.

132. Rosa micrantha Sm. Engl. bot. XXYV, t. 2490 (1812).

Im Voreikos-Thale bei Megaspilaeon. Höhe $700 \mathrm{~m}$.

133. Rosa dumetorum Thuill. Fi. Par. p. 250 (1799).

Var. solstitialis Bess. Prim. FI. Gal. I, p. 324 pro spec. (1809); Braun in Beck lil. Nicideröst. p. 795 (1892).

An Hecken bei Hagios Vlasios und Lopesi. Höhe 300-700 $\mathrm{m}$.

Var. amblyphylla Rip. apud 1)eségl. Cat. rais. in Bull. soc. roy. Belg. XV, p. 380 pro spec. (1876) Braun in Beck Fl. Niederöst. p. 795 (1892).

In der unteren Region des Chelmos bei Sudena. Höhe $1000 \mathrm{~m}$.

134. Rosa canina L. Sp. p!. p. 491 (1753).

Var. fissidens B orb. Primit. monogr. ros. in M. T. Akad. math. s természett. közl. AV1, p.413 (1880).

An Hecken bei Lopesi und Megaspilaeon. Höhe $300-700 \mathrm{~m}$.

135. Aremonia agrimonioides L. Sp. pl. ed. 2, p. 642 sub Agrimonia (1762); DC. Prodr. II, p. 588 (1825).

In der Tannenregion des Panachaicon und Olenos. Höhe $1400-1700 \mathrm{~m}$.

XYIII. LYTHRARIAE JUISS.

136. Lythrum Graefferi Ten. F1. Nap. Prudr. suppl. II, p. 27 (1811).

In Gräben bei Patras, häutig.

XIX. PARONYCHIEAE St. HiI.

137. Telephium orientale Boiss. Diagn. Pl. or. Ser. I, Nr. 10, p. 11 (1849).

Am Fusse der Kyllene bei Gura, höchst selten. Höhe $800 \mathrm{~m}$.

138. Herniaria incana L.am. Dict. Ill, p. 124 (1789).

In der oberen Region des Panachaicon. Höhe $1700 \mathrm{~m}$.

XX. SCLERANTHEAE Link.

139. Scleranthus verticillatus Tausch in Flora Xl1, Lrgänzbl. p. 50 (1829).

In der oberen Region des Panachaicon. Höhe $1500 \mathrm{~m}$.

\section{CRASSULACEAE I)(}

140. Umbilicus parviflorus Sibth. et Sm. F1. Grace. Prodr, I, p. 308 sub Cotylidone (1800); UC. Prodr. 1Ii, p. 400 (1828).

An Mauern der Acropolis von Patras.

141. Sedum laconicum Boiss. et Heldr. Liagn. P1. (1). Ser. I, Ni. 6, p. $55(1845)$.

An lielsen bei Ralavryta und auf dem Panachaicon. Höhe $800-1700 \mathrm{~m}$.

142. Sedum eriocarpum Sibtin. et Sm. Fl. Graec. Prodr. 1, p. $310(1806)$.

In der obcren Region des Panachaicon. Höhe $1700 \mathrm{~m}$.

143. Sedum rubens L. Sp. pl. p. 432 (1753).

1 der unteren Region des Olenos. Höhe $800 \mathrm{~m}$.

XXII. GROSSULARIEAE I)

14. Ribes Grossularia 1. Sp. pl. p. 201 (1753).

in der Tannenregion des Olenos und Chelmos. Höhe $1500 \mathrm{~m}$. 


\section{SAXIFRAGACEAE DC}

145. Saxifraga Sartorii Heldr. in Boiss. Fl. or. suppl. p. 248 (1888).

Var. erythrantha. Floribus purpureis.

Mit Ausnahme der Blüthenfarbe mit der Pflanze rom Delphi auf Euboea übereinstimmend.

Auf Kalkfelsen in der oberen Region der Kyllene oberhalb Gura, höchst selten. Höhe $2000 \mathrm{~m}$.

146. Saxifraga Friderici Augusti Bias. Viagg. per l'Istr. Dalm. e Nonten. p. 199 (1841).

in der oberen Region des Chelmos und der Kyllene. Höhe $2000 \mathrm{~m}$.

147. Saxifraga exarata Vill. Hist. pl. Dauph. 1II, p. 674 (1786).

In der oberen Region der Kryllene oberhalb Gura. Höhe $2000 m$.

148. Saxifraga graeca Boiss. et Heldr. in Boiss. Fl. or. II, p. 807 (1872).

In der Tannenregion des Panachaicon. Höhe $1400 \mathrm{~m}$.

149. Saxifraga tridactylites I. Sp. pl. p. 404 (1753).

In der oberen Region des Panachaicon. Höhe $1800 \mathrm{~m}$.

150. Saxifraga parnassica Boiss. et Heldr: Diagn. PI. or. Ser. II, Nr. 2, p, 69 (1856).

In der oberen Region des Chelmos und der Kyllene. Höhe 1200-2000 $\mathrm{m}$.

151. Saxifraga chrysosplenifolia Boiss. Diagn. Pl. or. Ser. I, Nt. 3, p. 20 (1843).

In der oberen Region des Panachaicon. Höhe $1600 \mathrm{ml}$.

\section{UMBELLIFERAE Juss.}

152. Ferula communis L. Sp. pl. p. 246 (1753).

In der Tannenregion bei Nlegaspilacon. Höhe $1000 \mathrm{~m}$.

153. Ferulago monticola Boiss. et Heldr. Diagn. Pl. or. Ser. II, Nr. 2, p. 91 (1856).

In Gebüschen bei Lopesi, Manesi und Kalavryta. Höhe 300-700 $\mathrm{m}$.

154. Ferulago nodosa L. Sp. pl. p. 334 sub Pincedano (1762); Boiss. Diagn. Pl. or. Ser. I, Nr. 10. p. 37 (1849).

In Gebüschen bei Lopesi. Hagios Vlasios, Megaspilaeon und Planideri. Höhe 300-700 $\mathrm{m}$.

155. Bonannia graeca L. Sp. pl. p. 252 sub Sio (1753). - Ferulu mudicaulis Spreng. Neue Ent. Il p. 149 (1821), non Nutt. - Laserpitun resinosum Pres1 Del. Prag. p. 137 (1822). - Bonamia resinifera Guss. Fl. Sic. Syn. 1, p. 355 (18+2). - Bonannia resinosa Strobl Fl. Etna in Öst. bot. Zeitschr.XXXIY' p. $174(1884)$.

Unter Gebüsch bei Lopesi. Höhe $400 \mathrm{~m}$.

156. Johrenia distans Griseb. Spic. Fl. Rum. et Bithyn. I, p. 374 sub Caroselino (1843). - J. graeca Boiss. et Sprun. in Ann. scienc. nat. 3 Ser. 1, p. 305 (1944).

In der Tannenregion bei Megaspilaeon, selten. Höhe $1000 \mathrm{~m}$.

157. Opopanax hispidus Friv. in Flora 1835 p. 333 sub Ferula; Griseb. Spic. Fl. Rum. et Bithyrn. I, p. 378 (1843). - O. orientale Boiss. in Ann. scienc, nat. p. 330 (1844).

Bei Megaspilaeon.

158. Malabaila involucrata Boiss. et Sprun. Ann. sciene. nat. p. 336 (1844).

In der Tannenregion des Olenos. Höhe $1400 \mathrm{~m}$.

159. Malabaila aurea Sibth. et Sm. Fl. Graec. Prodr. I. p. 192 sub Heracleo (1806); Boiss. Fl. or. II, p. $1053(1872)$.

Bei Psadopyrgos (leg. Grimburg), Lopesi und Megaspilaeon. Höhe 50-700 $\mathrm{m}$.

160. Oenanthe pimpinelloides L. Sp, pl. p. 255 (1753).

In Gebüschen hei Sarađi. Höhe $600 \mathrm{~m}$. 
161. Oenanthe incrassans Bory et (hauh. in Exp. scient. Mor. III, 2. p. 87 (1832). - (). incrussuta Boiss. Fl. or. 11, p. 956 (1872).

Sumpfige Orte bei Patras.

162. Scandix grandiflora 1. Sp pl. p. 257 (1753).

In der Tannen- und oheren kegion des Panachaicon, dann bei Kalarryta. Höhe $700-1700 \mathrm{~m}$.

lar. intermedia. Fructibus centralibus umbellularum longe patuleque hispidis, lateralibus scahridis

Mit der Grundform am Panachaicon und bei Kalavryta.

Var. lasiactina Boiss. Fl. or. 11, p. 917 (1872).

Auf dem Panachaicon.

163. Scandix australis L. Sp. pl. p. 257 (1753).

In der Tannenregion des Chelmos. Höhe $1200 m$.

164. Freyera macrocarpa Boiss. Ann. scienc. nat. 3. ser. 1I, p. 12 sub Butiniu (1St4); I)iagn. Pl. ur. Sier. 11, Nr. 2, p. 101 (1856).

Auf Lehmbergen bei Patras, selten.

165. Freyera parnassica Boiss, et Heldr. Diagn. Pl. or. Sel. Il, Nr. 2, p. 102 (1856).

Im Kalkschutte der oberen Region des Panachaicon und Chelmos. Höhe $1700-1900 \mathrm{~m}$.

166. Bulbocastanum ferulaceum Sibth. et Sm. FI. Graec. Prodr. 1, p. 186 sub Bunio (1806); Nyman Consp. Fl. Europ. p. 303 - Bumimm femlatolimm Desf. Choix pl. coroll. inst. Tournef. p. 55 (1808). - Bunimu divaricalmm Ces. in Linnaea Xl. p.31+ (1837), non Bert. - Camm diaricatum koch Syn. Deutsch. und Schweiz. Fl. p. 287 (18:38). - Carmm ferulacfolimm Bois.s. Diagn. Pl. or. Ser. 1. Nr. 10. p. 2'? (1819).

Auf W'iesen in der Tannenregion des Olenos. Höhe $1200 \mathrm{~m}$.

167. Carum multiflorum Sibth. et Sm. Fl. Graec. Prodr. 1, p. 188 sub Athamanla (1806): Bniss. Fl. or. II. p. 882 (1872).

Auf Kalkfelsen hei Megaspilaern. Höhe $800 \mathrm{~m}$.

168. Bupleurum trichopodum Boiss. et Sprun. in Ann. scienc. nat. 3. Ser. I, p. I 45) (ISt4).

In der 'lannenregion bei Sudena und Megaspilaeon.. Höhe 700 - $1000 \mathrm{~m}$.

169. Prangos ferulacea 1. Sp. pl. p. 35s suh Laserpitio (1762): Lindl. in Brandes Joum. P. 37 (1.925).

In der Tannenregion des Olenos. Höhe $1500 \mathrm{~m}$.

Vurde auch in jener der lyyllene von Heldreich gefunden.

170. Conium divaricatum Boiss. et Orph. Diagn. P1. (11. Ser. 11, Ni. 5, p. 103 (1856).

Auf Kalkfelsen bei Lopesi. Höhe $500 \mathrm{~m}$.

Wurde von Boissier in Fl. or. II, p. 922, meines Erachtens mit Unrecht, ils Varietät zu C. maculatum L. gezogen, denn abgesehen lon del 'lracht, unterscheidet es sich von dieser Art ganz ausgezeichnet Aurch die breiteren Blattzipfel, die nur 6-10strahlige Dolde, die viel längeren stark spreizenden Strahlen derselben, den Mangel eines Involucrums und die kleineren Blättchen des Involucellums.

171. Scaligeria cretica Urv. Enum. pl. Archip. p.33 sub. Bunio (1822): Vis. Fl. Dalm. I11, P. 70 (185'2). Auf Lehmhügeln bei Patras.

172. Smyrnium rotundifolium Mill. Gard. dict Nr. 22 (1759).

In der mnteren Region des Panachaicon, bei Lopesi, Lialavyta und Megaspilacon, meist truppenweise. 173. Eryngium multifidum Sibth. et Sm. FI Craec. Prodr. I. p. 175 (1806).

In der Tannenregion des Chelmos bei Sudena. llöhe $1100 \mathrm{~m}$.

174. Lagoecia cuminoides 1 .. Sp. p]. p. $20: 3$ (175:3).

Aul l.ehmhügeln bei Patras. 
XXY. CAPRIFOLIACEAE JuSS.

175. Lonicera etrusca Savi in Santi viatgg. I, p. 11:3 (1795).

Var. Roeseri Heldr. in Boiss. Diagn. Pl. or. Ser. 11, Nr. 2, p. $1(17(18).(j)$.

In der 'Tamnenregion des bei Megaspilaeon. Höhe $900 \mathrm{ml}$.

\section{RUBIACEAE Juss.}

176 Putoria calabrica 1. lil. Suppl. pl. p. 120 sub Asperula (1781); Pers. sy'n. 1, p. 524 (180. $)$.

In Felsritzen oberhalb der Bahnstation Dervenion. Höhe $400 \mathrm{~m}$.

177. Galium firmum '1'ausch in Flora XIV, p. 222 (1831). - G auremu. Vis. Ind. orto. but. Pad. P. $134(1842)$.

An Felsen bei Lopesi und Kalavryta. Höhe $400-700 \mathrm{~m}$.

178. Galium thymifolium Boiss. et Heldr. Diagn. Pl. or. Ser. 1, Nr. 6, p. 67 (1845).

In der Tannenregion des Chelmos oberhalb Planideri. Höhe $1300 \mathrm{~m}$.

179. Galum elongatum Presl Fl. sic. I, p. 59 (1826).

Sumpfige Orte bei Kalavryta. Höhe $700 \mathrm{~m}$.

180. Galium zacynthium Marg. et Reut. Essai d'une flore de l'île de Zante, p. 54 (1838).

In der Tannenregion des Panachaicon. Höhe $1000 \mathrm{ml}$.

181. Galium verticillatum Dant. in Lam. Dict. II, p. 585 (1789).

In der Tannenregion des Panachaicon und Chelmos. Höhe 1000-1200 $\mathrm{ml}$.

182. Valantia aprica Sibth. et Sm. Fl. Graec. Prodr. I, p. 90 sub Galio. (1806).

Im Felsenschutte des Chelmos bei Sudena. Höhe 1000-2000 m.

183. Asperula areadiensis Sims. in Curt. Bot. Mag. p. $2146(1820 \%$

In Felsentitzen ron Megaspilneon an durch das Voreikos-Thal bis Kalavryta, hier besonders aluf dem Berge Kastro sehr häufig; geht bis in die obere Region des Chelmos oberhalb Sudena. Höhe $700-2000 \mathrm{~m}$.

184. Asperula lutea Sibth. et Sm. F1. Graec. Prodr. 1, p. 88 (1806).

An Felsen im Voreikos-Thale bei Kalavryta. Höhe $700 \mathrm{~m}$.

185. Asperula arvensis L. Sp. pl. p. 102 (1753).

Bei Mlegaspilaeon. Höhe $500 \mathrm{~m}$.

\section{VALERIANEAE DC.}

186. Valeriana Dioscoridis Sibth. et Sm. Fl. Graec. Prodl. 1. p. 21 (1806).

lin der Tannenregion des Olenos. Höhe $1500 \mathrm{ml}$.

187. Valeriana olenaea Boiss. et Heldr. Diagn. Pl. or. Ser. II, Nr. 2, p. 118 (1856).

In der oberen Region der Kyllene oberhalb Gum, selten. Höhe 200 m.

188. Centranthus Sibthorpii Heldr. et Sart. in Boiss. Diagn. Pl. or. Ser. II, Nr. 2. p. 119 (1856).

In Felsen bei Lopesi und im Voreikos-Thale bei Kalavryta und Megaspilaeon. Höhe $400-i 00 \mathrm{~m}$.

189. Valerianella echinata L. Sp. pl. p. 47 sub Valerianu (1762); DC. Fl. fr. IV. p. 242 (1805).

An Rainen bei Kalarryta. Höhe $700 \mathrm{~m}$.

190. Valerianella truncata Reichenb. Pl. Crit. 11, p. I sub Fedia (182.4); Betcke Valer. p. 22 (1826). In der Tannenregion des Panachaicon. Höhe $1000 \mathrm{~m}$.

\section{DIPSACEAE Vaill.}

191. Morina persica L. Sp. pl. p. 28 (1753).

In der Tannenregion des Panachaicon, dann bei Kalavryta und Sudena. Höhe 700-1000 $\mathrm{m}$.

192. Pterocephalus plumosus 1. Mant. p. $14 \%$ sub Kinantia (1767); Coult. Dipsae. p. 3131 (1823).

In der Tannenregion bei Megaspilaeon. Höhe $800 \mathrm{~m}$. 
193. Pterocephalus Parnassi Spreng. Syst. 1, p. 384 (1825).

Im Voreikos-Thale bei Kalavryta und in der Tannenregion des Chelmos bei Sudena. Höhe $700-1$ '?ou $m$.

\section{COMPOSITAE Vaill.}

194. Senecio rupestris $\mathbb{W}$. et K. Pl. rar. Hung. II, p. 136 (1805).

In der Tannen- und oberen Region des Chelmos und Panachaicon. Höhe 1200-1800 $\mathrm{m}$.

195. Anthemis tinctoria L. Sp. pl. p. 896 (1753).

An Weingartenrändern bei Patras.

Var. pallida D C. Prodr. VI, p. 11 (18:37).

In der unteren Region bei Lopesi.

196. Anthemis Brachmanni Bo iss. et Heldr. Diagn. Pl. or. Ser. I, Nr. 6, p. 84 (1845).

In der Tannenregion des Panachaicon. Höhe $1000 \mathrm{~m}$.

197. Anthemis montana L. Sp. pl. p. 1261 (1762).

Var. Linnaeana Gr. et Godr. FI. Fr. 11, p. 155 (1850).

In der Tannenregion des Panachaicon und Chelmos. Höhe $1000-1500 \mathrm{~m}$.

198. Anthemis tomentosa L. Sp.pl. p. 893 (1753).

An den Ufern des Golfes von Corinth bei Rhion nächst Patras.

199. Achillea umbellata Sibth. et Sm. Fl. Graec. Prodr. II, p. 192 (1813).

In der oberen Region des Chelmos oberhalb Sudena. Höhe $2000 \mathrm{~m}$.

200. Achillea ligustica All. Fl. Ped. I, p. 181 (1785).

Bei Saradi, Megaspilaeon und Planideri. Höhe 700-1000 $\mathrm{m}$.

201. Matricaria Chamomilla L. Sp. pl. p. 891 (1753).

Häufig bei Patras, steigt hier bis in die Tannenregion des Panachaicun hinauf.

202. Filago spathulata Presl Del. Prag. p. 99 (1822).

In der Tannenregion des Olenos (f. decalians). Höhe $1000 \mathrm{~m}$.

203. Bellis perennis L. Sp. pl. p. 886 (1753).

Var. microcephala Boiss. Fl. or. III, p. 174 (1875).

In der oberen Region des Panachaicon, Chelmos und Ky yllene. Höhe $1800-2000 \mathrm{~m}$.

204. Evax pygmaea L. Sp. pl. p. 1311 sub Filagine (1762); Pers. Syn. III, p. 422 (1807).

Bei Patras.

205. Xeranthemum inapertum Willu. Sp. pl. 111, p. 1902 (1797).

Bei Kalavryta. Höhe $700 \mathrm{~m}$.

206. Onopordon argolicum Boiss. Diagn. pl. or. Ser. 1, Nr. 10, p. 91 (1849).

Wüste Plätze bei Corinth.

207. Onopordon illyricum L. Sp. pl. p. 827 (1753).

Bei Kalavryta. Höhe $700 \mathrm{~m}$.

208. Cynara Cardunculus L. Sp. pl. p. 827 (1753).

Bei Patras.

209. Chamaepeuce stellata 1. Sp. pl. p. 1153 sub Cardno (1753); DC. Prodr. VI, p. 658 (1837).

Bei Megaspilaeon. Höhe $7000 \mathrm{~m}$.

210. Chamaepeuce Afra Jacq. Hort. Schoenbr. 11, p. 180 sub Cardno (1797); DC. Prodr. VI, p. 659 (1837).

In der unteren und Tannenregion des Olenos, Chelmos und der Kiflene. Höhe 1000-1500 $\mathrm{m}$.

211. Galactites tomentosa Moench Meth. p. 558 (1794).

Bei Patras. 
212. Carduus taygeteus Buiss. ct. Heldr. I)iagn. pl. o1. Ser. II, Nr. 3, p. 12 (1856).

In der 'Tannenregion des Olenos. Höhe $1500 \mathrm{~m}$.

213. Jurinea glycacantha Sibth. et Sm. 19. Cracc. 1'rodr. 11, p. 156 sub Cirrduo (181:3): 1) C. P'rodr. Vl, р. 677 (1837).

In der Tamnenregion des Chelmos bei Megaspilaeon. Höhe $1000 \mathrm{~m}$.

214. Centaurea cana Sibth. et Sm. Fl. Graec. Prodr. II, P. 198 (1813).

In der oberen Region des Panachaicon und Chelmos. Höhe 1600-1800m.

215. Centaurea cyanus L. Sp.pl. p. 911 ( (1753).

In der unteren Region des Olenos bei Hagios Vlasios. Höhe $800 \mathrm{~m}$.

216. Centaurea Zuccariniana DC. Prodr. VI, p. 574 (I837).

Bci Sudena. Höhc $900 \mathrm{~m}$.

217. Centaurea hellenica Boiss. et Sprun. Diag1. pl. or. Ser. I, Nr. 6, p. 131 (1845).

In der unteren und Tannenregion des Panachaicon, Olenos und Chelmos rerbreitet. Höhe $700-1500 m$.

218. Centaurea calcitrapa L. Sp. pl. p. 917 (1753).

Bei Patras und Kalavryta.

219. Crupina crupinastrum Moris Enum. scm. hort. Laur. P. I2 sub Cintanvea (1841); Vis. Fl. Dalm. 11, F. $42(1847)$.

Bei Patras, Kalavryta und Megaspilaeon.

220. Hieracium Bauhini Schult. Observ. p. 164 (1809).

In der Tannenregion des Panachaicon. Höhe $100 \mathrm{~m}$.

221. Hieracium sabinum Seb. et Maur. Fl. Kom. Prodr. p. 270 (1818).

In der Tannenregion des Panachaicon und Olenos. Höhe $1400 \mathrm{~m}$.

222. Crepis Sieberi Bois s. Diagn. Pl. or. Ser. I, Nr. 11, p. 53 (1849).

In der Tannenregion des Panachaicon und Olenos. Höhe $1000 \mathrm{~m}$.

223. Crepis rubra L. Sp. pl. p. 806 (1753).

In der Tannenregion des Olenos. Höhe $1000 \mathrm{~m}$.

224. Crepis foetida L. Sp. pl. p. 807 (1753).

Bci Eglikada nächst Patras.

225. Crepis Dioscoridis L. Sp. pl. ed. 2, p. 1133 (1763).

In der unteren Region des Panachaicon und bei Lopesi.

226. Lagoseris bifida Vis. Stirp. Dalm. spec. p. 19 sub Trichocrenide (1826); Koch Syn. p. 435 (1838).

In der Tannenregion des Panachaicon. Höhe $1000 \mathrm{~m}$.

227. Tragopogon australis Jord. Catal. hort. Dijon. p. 32 (1848).

Bei Patras.

228. Tragopogon Samaritanii Heldr. et Sart. in Boiss. Diagn. 11. or. Ser. 11, Nr. 5, 1. 116 (1856). In der Tannenregion des Panachaicon, selten. Höhe $1000 \mathrm{~m}$.

229. Scorzonera crocifolia Sibth. et Sm. Fl. Graec. Prodr. II p. 123 (1813).

In der Tannenregion bei Megaspilaeon. Höhe $800 m$.

230. Scorzonera Jacquiniana Koch Syn. p. 425 sub Podospermo (1837); Boiss. F1. or. 111 , P. 757 (1875).

Var. messeniaca Bory et Chaub. in Exp. scient. Mor. III, 2. p. 231 pro specie (I832).

Auf Lehmbergen bei Patras, dann in der unteren und Tannenregion des Panachaicon, Olenos und Chelmos. Höhe $50-800 \mathrm{~m}$. 
bes starli verdickten 11 urzelstockes, der gedrängten mit kür\%eren Seitemipteln versehenen Blätter und der zumeist kürzeren nackten Stengel wegen von sehr auffälliger 'Jracht: gleichwohl von S. Jacunimikma (Ko 11$)$, zu welcher sie auch von Boissier als Synonym gestellt wird, durch kein wesentliches Merkmal verschieden. Der Pappus ist an den von mir untersuchten Exemplaren weiss, nicht schmutziggelblichweiss, wie an der mitteleuropäischen Pflanze.

231. Picris pauciflora IVilld. Sp. III, p. 1557 (1800).

In Macchien bei Patras.

232. Leontodon cichoraceus 'Te n. Prodr. Fl. Nap. F. NLI1, Fl. Nap. 11, p. 167 sub Apurgia (1811); Boiss. Fl. or. 111, p. T29 (1875. - Apargia fasciculata Bir. Manip. Il, t. 2 (1814).

In der Tannenregion des Panachaicon und Olenos. Höhe 1000-1200 m.

233. Leontodon graecus Boiss. et Heldr. Diagn. Fl. or. Ser. 1, Nr. 11, p. 39 (1849).

Auf Felsen bei Lopesi Höhe $400 \mathrm{~m}$.

234. Hypochaeris cretensis 1. Sp. pl. P. 1139 sub Soriola (1762); Bory et Chal ub. in Exp. scient. Nor. 111, 2-, p. 237 (1832)

In der unteren und Tannenregion des Olenos. Höhe 700-1300 $\mathrm{m}$.

\section{CAMPANULACEAE Juss.}

235. Campanula tomentosa l'ent. Choix pl. jard. Cels. t. 18 (1803).

An Felsen bei Megaspilaeon, im Voreikos-Thale, auf dem Berge Kastro bei kalarryta und bei Sudena. Höhe $700-1000 \mathrm{~m}$.

236. Campanula spathulata Sibtli. ct Sm. F1. Graec. Prodr. J, p. 137 (1806).

In der Tannenregion des Panachaicon, Olenos und Chelmos. Höhe 1000-1300 $\mathrm{m}$.

237. Campanula ramosissima Sibth. ct Sm. Fl. Graec. Prodr. 1. p. i37 (1806).

An Rainen bei Patras gemein.

238. Podanthum limonifolium Sibth. et Sm. F1. Graec. Prodr. 1, p. 114 sub Plytenmale (1806); Boiss. Fl. or. III, p. 95 (1875).

In Föhrenwäldern des Chelmos oberhalb Syrista. selten. Höhe $900 \mathrm{~m}$.

239. Specularia hybrida L. Sp. pl. p.168 sub Campannla (1753); DC. Nonogr. Campan. p.348 (1830). In der unteren Region des Panachaicon. Höhe $500 \mathrm{~m}$.

\section{OLEACEAE Lindl.}

240. Ligustrum vulgare L. Sp. pl. p. 7 (1753).

Bei Sudena und Planideri. Höhe $1000 \mathrm{~m}$.

\section{BORAGINEAE JuSs.}

241. Onosma angustifolium L.ehm. Asperif. p. 363 (1818).

In der Tannenregion des Panachaicon. Höhe $900 \mathrm{~m}$.

242. Alkanna Pulmonaria Ciriseb. Spic. Fl. Rum. et Bithyn. I, 1). 10 (184t).

In der Tamrenregion bei Mlegaspilacon. $110 \ddot{h}$ e $\$ 00 \mathrm{~m}$.

243. Onosma frutescens Lam. 111. 1, p. 407 (1791).

An Felsen bei Kalavryta und in Voreikus-Thale. Höhe $700 \mathrm{~m}$.

244. Myosotis cadmea Boiss. Diagn. Pl. or. Ser. 1, Nr. 11. 1) 122 (1819).

In der oberen Region des Panachaicon. Höhe $1700 \mathrm{~m}$.

245. Myosotis arvensis 1. Sp. pl. p. 131 pro var. . .11. scorpioides (1753); Koth Bot. Abhandl. p. 20 (1786). - M. internedia Link Enum. pl. hort. Berol. 1, p. 164 (18'21).

In der Tamnenregion des Panachaicon. Höhe $1000 \mathrm{~m}$. 
246. Myosotis hispida Schlechtend. in Mlag. Naturlr. Berlin, V111. p. 230 (1818).

Var. pygmaea Bert. Amoen. ital. I. p. 13 pro spec. (18I)).

In der oberen Region des Chelmos. Höhe $1800 \mathrm{~m}$.

247. Myosotis refracta Boiss. Voy. esp. p. 433 (1837).

In der wberen Region des Chelmos. Höhe $1800 \mathrm{~m}$.

\section{SCROFULARIACEAE Lindl.}

2-4. Verbascum macrourum Ten. Fl. Nap. III, p. 216 (1811).

In der Tamenregion des Panachaicon. Höhe $1000 \mathrm{~m}$.

Von I: thapsiforme Schrad., zu welcher Art I: macronrum von Boissier als Synonym gestellt wird, lurch die dicke, äusserst gedrungene und sehr dicht filzige Trauhe und durch Staubfäden, Welche mehrmal länger sind als die herablaufende Anthere, verschieden.

2-40. Verbascum Sartorii Buiss. et Heldr. Diagn. Pl. or. Ser. 1, Nr. 7, p. $38(1846)$.

Bei Kalaverta. Höhe $700 \mathrm{~m}$.

2.70. Verbascum plicatum Sibth. et Sim, Fl. Graec. Prodr. L. p. 150 (1800).

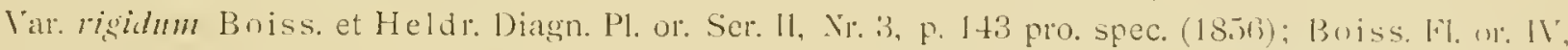
F. 312 (1879).

Bei Patras und Derrenion.

25). Celsia Daenzeri Fauché et ("haub. in Exp. scient. Mor. III, 2, p. 342 (18322).

Bei Mlegaspilaeon. Höhe $800 \mathrm{~m}$.

Ich habe diese Art hier in grosser. Anzahl beobachtet. Sie wird bis anderthalb Neter hoch und ist der grossen goldgelben, am Morgen sich öfnenden Blüthen wegen eine Zierde der V'egetation. Die ron mir in der Öst. bnt.Zeitschr. 1890, p. 405 aufgestellte C. roripifolia aus Bulgarien soll nach Velenovský Fl. Bulg. p. 418 mit ihr identisch sein. Die Unterschiede bejder Arten sind aber derart aulfällig, insbesondere ist. nebst den übrigen a. a. O. hervorgehobenen Merkmalen, die Form der Deckblätter constant so verschieden, dass dieselben unmöglich vereinigt werden können. Hiezu kommt noch, dass die Corollen der C. Dacnzcri fast noch einmal so gross sind, als jene der C. roripifolia.

252. Celsia acaulis Bory et Chaub. in Exp. scient. Mlor. III, 2, p. 177 (1832).

Auf den höchsten Erhebungen des Chelmos oberhalb Sudena. Höhe $2300 \mathrm{~m}$. Von Boissier nur oberhalb des Styx angegeben.

253. Digitalis ferruginea L. Sp. pl. p. 622 (1753).

In der Tannenregion des Olenos und der hyllene. Höhe $1200 \mathrm{~m}$.

254. Veronica thymifolia Sibth. et Sm. Fl. Graec. Prodr. I. p. 6 (1806).

In Polstern von Astrugalus angustifolius an der südlichen Abdachung des Gipfels der Kyllene. Höhe $2350 \mathrm{~m}$.

Eine durch die kriechenden Stämmchen, die kurze dichte Behaarung aller Theile, die gedrängtstehenden linealen, umgeroliten, ganzrandigen Blätter, die kurze, dichte, liopfartige Traube und die kleinen rosenrothen Blüthen ausgezeichnet charakterisirte Art

Die rorliegenden Exemplare stimmen volliommen mit der Cretenser Pllanze rom Lalssiti-Gebirge üherein.

Ich zweifle, dass diese Art bislang ausserhalb Creta's gefunden wurde, obzwar Boissier in der Flora or. IV, p. $44+$ dieselbe auch am Tymphrestus, Parnass und Olymp wachsend angibt. Höchstens könnte die Pllanze des Pamasses, von Welcher ich keine Exemplare sah und über welche Boissier auch nichts Näheres angibt, zu 1'. thymifolia gehören; jene von den beiden anderen exwähnten Bergen aber sicher nicht. Die Tymphrest-Pflanze, welche als 1'.tymphrestea Boiss. ct Sprun. in Boiss. Diagn. Pl. or. Ser. 1, 
Nr. t. p. 7 , beschrieben wurde, kenne ich zwar nicht aus eigener Anschauung, sie kann aber der Diagnose nach, in welcher es heisst "foliis superne obsoletissime et acutiuscule denticulatis, corolla coerulea calyce duplo longiori «, und von welcher Boissier weiter unten sagt: "I. prostruta facie melius refert ", unmöglich mit 1: Ihymifolia identiticirt werden. Ich vermuthe übrigens, dass sie dieselbe Pflanze sei, welche ich als T. prostrata var. filicaulis in Verh. zool. bot. Ges. 1888, p. 761 ron der Kiona beschrieb.

Zweifellos verschieden von ihr ist aber die Pflanze des Olymps, welche als $V$ tencrioides Boiss. et Heldr. I. c. Ser. II, Nr. 3, p. 169 beschrieben wurde, und von welcher ich Originale besitze. Sie ist von I: thymifolia durch fädliche, zweizeilig behaarte Stengel, grössere, kahle, flache, entferntstehende, gekerbte Blätter, kahle Kelche, grasse, blaue Corollen und kahle Kapseln himmelweit verschieden, und es bleibt gänzlich räthselhaft, wieso sie Boissier später in der Flora or. mit l: thlmifolia vereinigen konnte. Sie gehört meiner Ansicht nach in den Formenkreis der 1: prostrata L.

255. Veronica arvensis L. Sp. pl p. 13 (1753).

In der oberen Region des Panachaicon. Höhe $1600 \mathrm{~m}$.

256. Veronica peloponnesiaca Boiss. et Orph. in Boiss. Fl. or. IV, p. 462 (1879).

In der unteren Region des Chelmos bei Kalavryta und in der mittleren Region des Panachaicon. Höhe $700-1500 \mathrm{~m}$.

257. Veronica panormitana Tin. in Guss. Fl. Sic. Prodr. suppl. I, p. 4 (1832).

In der 'Tannenreginn des Panachaicon. Höhe $1200 \mathrm{~m}$.

\section{OROBANCHEAE JuSS.}

258. Orobanche Spruneri F. IV. Schultz in Flora XXVI, p. 130 (1843).

Bei Kalavryta. Höhe $800 \mathrm{~m}$.

2.59. Orobanche minor Sutt. in Trans. Lin. soc. IV, p. 178 (1797).

Vall, adenostyla Vis. Fl. Dalm. II, p. $179(18+7)$. - O. livida Sendtn. Herb. fide Vis. I. c.

lVeingartenrainder bei Patras.

\section{XXXY. LABIATAE JuSS.}

260. Teucrium prostratum Schur. Sert. p. 60 (1853). - T. montanmm i. hirsutmm Boiss. Fl. or. IV, P. $819(1879)$.

In der Tannentegion des Panachaicon und Olenos. Höhe $1000 \mathrm{~m}$.

261. Ajuga orientalis T. Sp. pl. p. 561 (1753).

In der Tannenregion des Olenos. Höhe $1200 \mathrm{~m}$.

262. Salvia Barrelieri 'Ten. Fl. Nap. I, p. 9 (1811).

An Wegrändern bei lliospulos, südlich von Patras und bei Kalavryta. Höhe $100-700 \mathrm{~m}$.

Eine durch die zahlreichen hellazurblauen Blüthen höchst auflälige Art, welche in Griechenland, wic es scheint, ziemlich verbreitet ist, von den Bearbeitern der griechischen Flor:a und auch anderen Autoren aber entweder nicht gekannt oder verkannt und für S. pratensis L. gehalten wurde. Die Confundirung mit letztgenannter Art dürftc hauptsächlich dem Umstande zuzuschreiben sein, dass bei nicht sorgfältigem Trocknen der Exemplare der für den ersten Blick auffülligste Charakter, die himmelblaue Farbe der Blüthen verloren geht und dieselben dann thatsächlich habituell der S. pratensis L. sehr ähnlich werden. Nebst liesem Merkmale in der Blüthenfarbe sind aber noch bei genauerer Untersuchung andere constantbleibende vorhanden, welche die Art ganz präcise unterscheiden lassen. Ich gebe dieselben mit Bezug auf jene ron S. pratensis in nachfolgender Tabelle: 


\section{S. matcusis 1. .}

Stengel cinfach oder ästig, feinflaumig his zottig.

Bläitter verhältnissmäissig klein, dic stengelständigen kurzgestielt, das oberste Paar sitzend.

Deckblätter eiförmig zugrespitzt.

Kelch stieldrüsig.

Blumenkronen violett, stieldrüsig.
S. Barreliori Ten.

Stengel meist ausgebreitet-ästig, von kurzen steifen Haaren rauly.

Bläitter verhältnissmässig gross, die stengelständigen sitzend oder die oberen Paare breit zusammengewachsen.

Deckblïtter rundlich nit kurzer aufgesetzter Spitze. Kelch liurzharig, gelbdrüsig punktirt.

Blumenkronen hell azurblau, gelbdiüsig punktirt.

Nebst den oben angeführten Standorten besitze ich S. Burrlicri aus Griechenland noch aus dem centralen Peloponnes von Zatuna (leg. Orplianides) und von Aetolikon in Actolien (leg. Heldreich), beide mit der Etiquette S. matensis L. var.

$\mathrm{Ob}$ Linné unter seiner S. llacmatodes die gleiche Art verstand, ist schwer zu sagen. Linné crtirt in Spcc. pl. p. 24 (1753) nebst anderen Barrelier Pl. Gall. Hisp. ct 1tal. (1714), wo auf Seite 25 eine Peschreibung und auf Tab. 185 eine Zeichnung zu finden ist, welche immerhin in Einklang mit unserer Pflanze zu bringen sind, von welchen jedoch nicht mit absoluter Sicherheit gesagt werden kann, dass sie mit S. Barrelicri Ten. identisch sind. Auch in Parl. Fl. Ital. VI, p. 250 ist S. hacmatodes L. num mit Fragezeichen als Synonym der S. Barrelicri Ten. erwähnt. Unter solchen Umständen zog ich es vor, den Tenore’schen Namen anzuwenden, welcher Autor die Art a. a. O. ausführlich beschrieben und vorzüglich abgebildet hat, so dass kein Zweifel über dieselbe Platz greifen kann. 'Tenore gibt zwar als Vaterland seiner Art nebst Süditalien auch Spanien an, was jedoch unrichtig ist, da die spanische Pflanze, welche von Etlinger (1777) ebenfalls mit dem Namen S. Burelicri belegt Wurde $=$ S. Mnamocua Vahl. Enum. I p. $269\left(180 \cdot \frac{1}{1}\right)$ ist.

Dem Prioritätsgesetzc nach nüisste letztere eigentlich als Synonym zu S. Barrclicri Etling. gestellt werden und folglich die Tenore'sche S. Barelieri einen neuen Namen erhalten. Insolange jedoch die S. hameatodes L. nicht aufgeklärt ist, welche, wie oben erwähnt, nöglicherweise mit S. Barclieri Ten. zusammenfällt, möchte ich nicht diesc Consequenz gezogen haben, umsoweniger als in S. Tcnorii Spreng. Syst. veg. I, p. 65 (1825) höchst wahrscheinlich schon ein Nane für dieselbe existirt.

263. Salvia virgata Ait. Hort. Kew. I, p. 39 (1789).

In der unteren Region bei Patras, Kalavryta und Planideri. Höhe $30-700$ m.

264. Salvia peloponnesiaca Boiss. et Heldr. Diagn. Pl. or. Ser. 1, Nr. 7, p. 47 (1846).

An Grasplätzen und Weingartenrändern bei Patras.

265. Ziziphora capitata L. Sp. p. p. 21 (1753).

In der unteren Region bei Kalarryta. Höle $700 \mathrm{~m}$.

266. Scutellaria peregrina L. Sp. pl. p. 699 (1753).

Var. Sibthorpii Benth. in DC. Prodr. XII, p. 419 pro rar. S. Colmmmac All.; Boiss. et Reut. Diagr. Pl. or. Ser. I1, Nr. 4, p. 28 (1895).

Am Fusse der liyllene bei Gura. Höhe $700 \mathrm{~m}$.

267. Prunella laciniata L. Sp. pl. p. 600 pro var. P. zulgaris (17.33); L. Sp. pl. ed. 2, p. 837 (1763). - P. alba Pall. in M. a Bieb. Fl. Taur. cauc. II, p. 67 (1808).

Grasige Abhänge bei Patras. Höhe $50 m$.

268. Melittis melissophyllum L. Sp. pl. P. 597 (1753).

In Föhrenwäldern des Chelmos bei Planideri und Syvista. Höhe $900-1000$ m.

269. Lamium nivale Boiss. et Heldr. Diagn. Pl. or. Ser. 1, Ni: 7, p. 54 (1846).

ln der oberen Region des Panachaicon und Chelmos. Höhe $1200-2000 \mathrm{~m}$.

(v. Haläcsy.) 
270. Stachys cretica L. Sp. pl. p. 581 (175:3).

Bei Patras und Megaspilaeon. Höle $20-700 \mathrm{~m}$.

Var. albiflora.

In der Tannenregion bei Megaspilaeon. Höhe $800 \mathrm{~m}$.

271. Stachys graeca Boiss. et Heldr. Diagn. Pl. or. Ser. I, Nr. 12, p. Ti (IS53).

In der Tannenregion des Olenos. Höhe $1200 \mathrm{~m}$.

272. Stachys Parolinii Vis. 1llustr. di alc. piante della Graec. p. Lo (1842).

Auf Kalklelsen im Voreikos-Thale bei Megaspilaeon. Höhe $700 \mathrm{~m}$.

273. Phlomis fruticosa L. Sp. pl. p. 584 (1753).

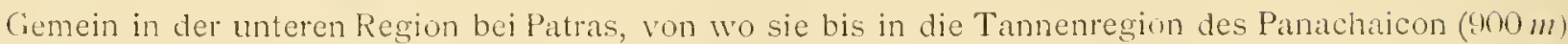
hinaufsteigt, auch am Olenos, dann bei Kalavryta und im Voreikos-Thale.

27. Phlomis samia L. Sp. pl. p. 585 (1753).

In der Tannenregion des Chelmos bei Planideri und IIegaspilaeon. Höhe $800-1000$.

275. Ballota acetabulosa L. Sp. pl. p. 58t sub Marmbio (1753); Benth. Labiat. p. 595 (1832 - 36).

In der unteren Region im Voreikus-Thale, bei Kalarryta und Planideri. Fehlt in der Ungebung ron Patras.

276. Calamintha suaveolens Sibth. et Sm. Fl. Graec. Prodr. I, p. 420 sub Tly'mo (1806); Boiss. Fl. or. $N$, p. $482(1879)$.

In der Tannenregion bei Megaspilaeon. Höhe $900 \mathrm{~m}$.

277. Calamintha alpina L. Sp.pl. p. 591 sub Thymo (1753); Lam. Fl. fr. II, p. 394 (1778).

In der Tannenregion des Chelmos oberhalb Planideri und in der Gipfelregion der Kyllene. Höhe 1500 bis $237+m$.

278. Thymus atticus Čelak. in Flora LXY, p. 56+ (1882). - T. striatus Boiss. Fl. or. IV, p. 557 pru parte (1879), non Vahl.

Im Voreikus-Thale bei Megaspilaeon. Höhe $700 \mathrm{~m}$.

279. Thymus Chaubardi Boiss. et Heldr. Diagin. Pl. or. Ser. II, Nr. 4, p. 6 (1859).

In der unteren und Tannenregion des Chelmos bei Sudena. Höhe 1000-1200 $\mathrm{m}$.

\section{LENTIBULARIEAE Rich.}

280. Pinguicula hirtiflora Ten. Fl. Nap. 11t, p. Is (1811).

An nassen Felsen in Föhrenwäldern bei Zachuli. Höhe $800 \mathrm{~m}$.

\section{PRIMULACEAE I ${ }^{\top} \mathrm{ent}$.}

281. Lysimachia atropurpurea L. Sp. pl. p. 137 (175:3).

Häufig an den sandigen Ufern des Sumpfes bei Kalavryta, auch an Wegräindern bei Manesi. Höhe $700 \mathrm{ml}$

282. Primula acaulis L. Sp. pl. P. 143 pro var. P. veris (1753); Ja cq. Mlisc. I. p. 1.,8 (17-8).

An Bachufern am Fusse des Chelmos bei Syvista. Höhe $700 \mathrm{~m}$.

\section{GLOBULARIEAE DC.}

283. Globularia stygia Orph. in Buiss. Diagn. Pl. or. Ser. 11, Nr. 4, p. 60 (1859).

In Felsenritzen der oberen Region des Chelmos oberhalb Sudena, höchst selten. Höhe $2000 \mathrm{~m}$.

XXXIX. PLUMBAGINEAE Endl.

281. Armeria undulata Bory et Chaub. in Fxp. scient. .10r. I11, 2, p. 93 sub Statice (1832); Bois s. i.1 1) C. Prodr. Nill. p. (39.) (18).

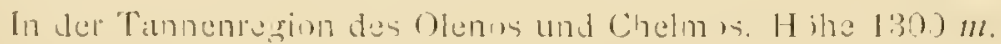




\section{PLANTAGINEAE Juss.}

285. Plantago humilis Jan. Elench. pl. p. $3(1826)$.

In der Tannenregion der kiyllene uberhalb Crura. Höhe $1000 \mathrm{~m}$.

286. Plantago graeca Hal. in Verh. zool. hot. Ges. Wien, XXXY'll1, p. 6131 (1888).

In der oberen Regioa des Panachaicon, Chelmos und der Kyllene, stellenweise ansehnliche libehen beleckend. Höhe $1800-2200 \mathrm{~m}$.

287. Plantago Bellardi Mll. Fl. Ped. 1, p. 82 (1785). P. pilosa Pourr. in Act. Tolos. Ill, p. 324 (1788). Grasplätze bei Patris.

\section{SALSOLACEAE Moq.}

288. Atriplex Halimus 1.. Sp. pl. p. 1052 (1753).

Auf sandigen Hügeln bei l'atras.

\section{XliI. POLYGONEAE Juss.}

289. Rumex graecus Boiss. et Heldr. Diagn. Pl. or. Ser. 11, Nr. t, p. 80 (1859).

Auf wüsten Plätzen in Sudena. Höhc $1000 \mathrm{~m}$.

290. Rumex conglomeratus Murr. Prodr. stirp. Gotting. p. 52 (1770).

An Wegrändern bei Patras.

\section{EUPHORBIACEAE JuSS.}

291. Euphorbia myrsinites 1. Sp. pl. p. 461 (1753).

In Voreikos-Thale. Höhe $700 \mathrm{~m}$.

\section{URTICACEAE Endl.}

292. Urtica dioica L. Sp. pl. p. 984 (1753).

Bei Patras.

\section{XI.V. CUPULIFERAE Rich.}

293. Quercus Farnetto Ten. Cat. Nap. p. 65 (1819).

ln der unteren Region des Olenos und bei Mlanesi. Höhe $700-800 \mathrm{~m}$.

294. Ostrya carpinifolia Scop. Fl. Carn. ed. 2, p. $244(1772)$.

In der unteren Region fles Chelmos oberhalb Syrista. Höhe $800 \mathrm{~m}$.

LXVI. SALICINEAE Rich.

295. Salix purpurea L. Sp.pl. p. 1017 (1753).

Bei Kalavryta. Höhe $700 \mathrm{~m}$.

296. Salix amplexicaulis Bory et Chaub. in Exp. scient. Mor. HI, 2, p. 277 (1832).

An Bachrändern bei Eglikada am Fusse des Panachaicon südlich ron Patras und bei Kalarryta. H. 100-700 m. Bei letzterem Orte schon von Heldreich gesammelt (vergl. Boiss. Fl, or. 11. p. 1187).

Diese Weidenart wurde zuerst von Bory und Chaubard a. a. O. im Jahre 1832, nicht wie Fritsch in Gartenfl. 43. Jahrg., p. 39 meint, im Jahre 1838 in Flor. Pelop. beschrieben und auf der Tafel XXX1V naturgetreu abgebildet. Da die genannten Autoren jedoch nur Exemplare ohne Blüthen vor sich hatten. so konnte die Beschreibung ihrer neuen Art nur eine mangelhafte sein; und da sie weiters selbst in einer der Diagnose angefügten Bemerkung hervorhoben, dass sie anfänglich im Zweifel waren, ob die ihnen vorliegenden Zweige thatsächlich einer Weidenart angehörten, so ist es erklärlich, dass Decennien hindurch eine Unklarheit über die Pflanze unter den Autoren herrschte, und dass dieselbe selbst bis heute noch nicht vollständig aufgeklärt wurde. So sagt Steudel im Nomenclator bot. II, p. 494, „S. amplexicanlis est: Apocynum venetum!! (sec. Buchinger in litt.) " Dieser Ansicht schliesst sich auch Andersson in seiner 
Weidenmonographie an. Erst im Jahre 1879 wurde ron Boissier in Fl. or. N1, p. 1187 , die Pflanze richtig wieder ats Weide declarirt (»certissime salicis species") und als Varietät zu S. purpurea L. gestellt. ln jüngster Zeit hat Dieck endlich in der Gartenfl., 42. Jahrg., p. 674 (1893) nachzuweisen versucht, dass S. amplexicaulis identisch mit S. oppositifolia Host sei und bemerkt, dass er dieselbe im Gebiete des Sardagh und weiters in Nordmacedonien angetroffen habe. Dieser Ansicht ist jedoch bald darauf Fritsch a. a. O. die S. amplexicaulis für eine von S. oppositifolia verschiedene Art erklärend, entgegengetreten. Ich weise auf diese Publication Fritsch's, mit dessen 1nhalt ich mich rollständig einverstanden erkläre, hin. Hinzufügen möchte ich nur noch, dass ich bezweifle, dass S. amplexicaulis im Gebiete des Sardagh oder anderswo in Macedonien vorkomme, und dass Dieck daselbst wohl nur S. oppositifolia angetroffen haben dürfte, da es doch in diesem Falle anzunehmen wäre, dass sie auch in Nittelgriechenland und Thessalien bereits aufgefunden worden wäre. Weder die älteren Floristen, noch Heldreich, der doch am meisten diese Gebiete durchstreifte, haben jedoch S. amplexicaulis daselbst irgendwo beobachtet. Auch ich sah sie hier während meiner Reisen in den Jahren 1888 und 1893 nirgends, sondern fand sie nur im Peloponnes. Bei Kalavryta zwar leider auch nur in Blättern, bei Eglikada an Fusse des Panachaicon, jedoch mit im vorgeschrittenen Stadium befindlichen weiblichen Kätzchen.

Nachfolgend gebe ich einc nach diesen Exemplaren entworfene Beschreibung der Art und möchte nur noch betonen, dass diese, was die Blätter anbelangt, fast congruent mit der oben citirten Abbildung Bory und Chaubard's zu nennen sind:

Fruticosa vel arborescens, ramis vimineis tenuibus rubescentibus, junioribus caesio-pruinosis; foliis oblongis oblongo-lanceolatisve, acuminato-apiculatis, glabris, subtus caesiis, basi rotundato-truncatis vel cordato-amplexicaulibus, marginibus minute denticulatis; amentis femineis lateralibus, praecocibus, sessilibus, oppositis, erecto-patulis, breviter cylindricis, densifloris, basi bracteatis; squamis obovatis, villosis, apice fuscis; capsulis ovato-conicis, obtusis, tomentosis, sessilibus; stigmatibus subsessilibus, divergentibus.

Dass die Pflanze also thatsächlich eine Weide ist, steht ausser jeder Frage; aus dieser Beschreibung ergibt sich aber auch weiters zur Evidenz, dass sie, wie dies schon Boissier vermuthete, zur $S$. purpurea L. in nächster Beziehung steht. Trotz des sehr ähnlichen Fruchtbaues kann sie jedoch meines Dafürhaltens der Bereifung und der höchst charakteristischen Blattgestalt wegen nicht als Varietät zu dieser gestellt werden, sondern muss vielmehr als selbständige Art aufgefasst werden. Die Unterschiede liegen vorderhand, da die männlichen Kätzchen noch immer unbekannt sind, freilich nur in den vegetativen Theilen; dieselben sind jedoch so prägnant, dass beide Arten unmöglich zu einer Art cumulirt werden können. Insbesondere auffällig sind bei S. amplexicaulis die mit einem intensiven hechtblauen Reife überzogenen Triebe und die opponirten, besonders an letzteren mit breiter stengelumfassender Basis sitzenden, jenen von L. implexa oft nicht unähnlichen Blätter, welche oft bei einer Länge von $3-4 \mathrm{~cm}$ eine Breite von $2.5 \mathrm{~cm}$ erreichen. Offenbar haben auch Bory und Chaubard solche Stocktriebe geschen, da sie sagen «folia oblongil obtusissiman.

297. Salix incana Schrank Baier. 11. 1, p. 230 (1789).

An den Ufern des Voreikos potamos bei Kalavryta. Höhe $700 \mathrm{~m}$.

\section{ORCHIDEAE Juss.}

298. Cephalanthera alba Crantz Stirp. Austr. ed. 2, V1, p. 460 sub Epipactide (1769); Fritsch in Üst. bot. Zeitschr. XXYVIll, p. 81 (1888). - C. pallens Rich. Orchid. amont. p. 38 (18+7).

In der Tannenregion des Panachaicon und des Chelmos bei Sudena. Höhe 1000-1100 $\mathrm{m}$.

299. Orchis quadripunctata Cyr. in Ten. Prodr. Nap. p. 53 (1811).

In der Tannenregion des Panachaicon. Höhe $1000 \mathrm{~m}$.

300. Ophrys cornuta Ste 1 in M. a Bieh. Fl. Taur. Cauc. 11, p. $37 n$ (1808).

Auf sandigen Hügeln und an Weingartenrändern bei Patras nicht selten. 


\section{IRIDACEAE Lindl.}

301. Iris Sintenisii Janka Adat. Erd. p. 173 (1876).

In Büschen von Qnercus coccifera in der unteren Region der Kyllene nächst Gura. Höhe $800 \mathrm{~m}$.

302. Crocus Sieberi Gay in Bull. fer. XXY, p. 220 (1831). - C. nivalis Bory et Chatub. in Exp. scient. Mor. III, 2, p. 21 (1832).

An Schneefeldern des Panachaicon und Chelmos. Höhe $1800-2000 m$.

\section{IL. AMARANTACEAE Juss.}

303. Sternbergia colchiciflora W. et K. PI. rar. Hung. II, p. 172 (1805).

In der oberen Region des Chelmos oberhalb Sudena sehr selten. Höhe $1600 \mathrm{~m}$.

\section{LILIACEAE DC.}

304. Anthericum Liliago L. Sp. pl. p. 310 (1753).

In Föhrenwäldern des Chelmos oberhalb Syvista selten, Höhe 1000 m.

305. Fritillaria Guicciardii Held1. et Sart. in Boiss. Diagn. Pl. or. Ser. Il, Nr. 4, p. 102 (1859).

Auf dem Gipfel der Kyllene sehr selten. Höhe $2374 m$.

306. Tulipa ąustralis Link. in Sehrad. Journ. 11, p. 317 (1799).

Var. montana kunze in Flora 1846, p. 637 pro var. T. silvestris; Willk. Prodr. FI. Hisp. 1, p. 219 (1870); Levier les Tulip. europ. p. 104 (I884).

In der oberen Region des Chelmos und der Kiyllene selten. Höhe $2000-2200 \mathrm{~m}$.

307. Gagea arvensis Pers. in Usteri Ann. XI, p. 8 sub Ornithogalo (179 ) ; Roem. et Schult. Syst. VII, p. $5+7$ (1829).

In der Tannenregion des Chelmos. Höhe $1500 \mathrm{~m}$.

308. Ornithogalum fimbriatum Willd. in Neu. Verh. nat. Fr. Berlin Ill. p. 420 (I801).

In der Tannenregion des Panachaicon, sehr selten. Höhe $1100 \mathrm{~m}$.

309. Ornithogalum montanum Cyr. in T'en. FI. Nap. I, p. 176 (1811).

In der oberen Region des Chelmos oberhalb Sudena. Höhe $1700 \mathrm{~m}$.

310. Ornithogalum tenuifolium Guss. Prodr. FI. Sic. I. p. 413 (I 827).

In der Tannenregion des Panaehaicon. Höhe $1000 m$.

311. Ornithogalum oligophyllum CIarke Travels in var. countr. of Eur., Asia and Afr. II, 3, p. 555 (1816).

In der oberen Region des Chelmos oberhalb Sudena. Höhe $1800 \mathrm{~m}$.

Durch kürzere Blüthenstiele von der Balkanpflanze verschieden.

312. Scilla bifolia L. Sp. pl. p. 309 (1753).

Var. nivalis Boiss. Diagn. Pl. or. Ser. 1, Nr. 5, p. 63 pro spec. (1844); Fl. or. V, p. 227 (1881).

An Schneefeldern des Chelmos. Höhe $2000 \mathrm{~m}$.

313. Muscari Heldreichii Buiss. Diagn. Pl. or. Ser. II. Nr, 4, p. 109 (1859).

In der oberen Region der Kyllene. Höle $2000 \mathrm{~m}$.

314. Allium trifoliatum Cyr. Pl. rar. II, P. 11 (1792).

In der Tannenregion des Olenos. Höhe $1000 \mathrm{~m}$.

\section{JUNCACEAE BartI.}

315. Juncus glaucus Ehrh. Beitr. VI, p. 83 (1790).

Bei Kalavryta. Höhe $700 \mathrm{~m}$. 
316. Luzula nodusola Bory ct ('haub. In Exp. scient. Mor. 111. 2. p. 105 sub lmino (1832); E. May

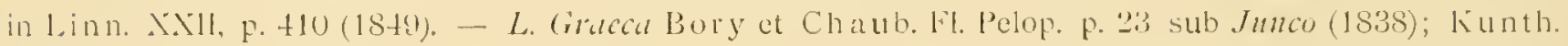
Enum. 111, p. 3101841.

ln der Tannenregion bei Megaspilaeun. Höhe $800 \mathrm{~m}$.

317. Luzula Forsteri Sm. F1. Brit. 11I, p. 9395 sub Jumco (1804); DC. Syn. Fl. Gall. p. 150 (1806). In der Tannenregion des Panachaicon. Höhe $1200 \mathrm{~m}$.

\section{AROIDEAE Juss.}

318. Arum Italicum 11 ill. Dict. ed. 8 Nr. 2 (1768).

In Gebüschen bei Patras.

\section{CYPERACEAE Juss.}

319. Carex laevis kit. in Willd. Sp. pl. IV, p. 292 (1805.

Auf dem Gipfel der Kyllene, selten. Höhe $2374 \mathrm{~m}$.

320. Carex macrolepis D C. Cat. hort. Nonspel. p. 89 (1813).

In Föhrenwäldern des Durduvana-Sattels oberhalb Syvista, selten. Höhe $1200 \mathrm{~m}$.

\section{GRAMINEAE JuSS.}

321. Sesleria coerulans Friv. in Flora X1X, p. 438 (1836).

In der oberen Region der Kyllene oberhalb Gura. Höhe $2000 \mathrm{~m}$.

32:- Avena filifolia Lag. Elench. p. 4 (1816). - A. convoluta Presl Cyp. et Gram. sic. p. 31 (1820). - A. fallar Ten. F1. Nap. 111, p. 96 (1824). - A striata Vis Fl. Dalm. 1, p. 70 (1842). - A. Heldreicliii Parl. H. Palerm. I, p. $111(18+5)$.

In der Tannenregion des Panachaicon. Höhe $1000 \mathrm{~m}$.

32:3. Aira capillaris Host. Gram. IV, p. 20 (1809).

In der Tannenregion des Panachaicon. Höhe $1000 \mathrm{~m}$.

32.4. Melica uniflora Retz. Observ. 1, p. 10 (1779).

In Föhrenwäldern am östlichen Abhange des Chelmos oberhalb Syvista. Höhe $800 \mathrm{~m}$.

325. Dactylis glomerata L. Sp. pl. p. 71 (1753).

Var. hispanica Roth Cat. bot. 1, p. 8 pro spec. (1797); Boiss. Fl. or. V, p. 596 (1884).

Bei Patras, auf dem Panachaicon und Chelmos bei Kalavryta und Sudena, steigt bis in die Tannenregrion. Höhe $50-1200 \mathrm{~m}$.

326. Bromus matritensis L. Am. acad. IV. p. 265 (1750).

Bei Patras.

327. Bromus intermedius Guss. Prodr. Sic. I, P. 114 (1827).

Auf sandigen Hügein bei Patras.

328. Bromus squarrosus L. Sp. pl. P. 76 (1753).

In der unteren und Tannenregion des Panachaicon. Höhe $500-1000 \mathrm{~m}$.

320. Festuca laevis Hack. Mon. Fest. curop. p. 107 pro subspec. Fo ovina (1882).

Var. Heldreichii Hack. 1. c. p. p. 109 pro subvar. F. ozinac.

In der unteren Region des Chelmos bei Sudena. Höhe $1000 \mathrm{~m}$.

330. Festuca valesiaca Schleich. in Gaud. Agrost. helv. I, p. 242 (1811).

Bei Megaspilaeon und Hagios Vlasios. Höhe $700 \mathrm{~m}$.

331. Poa alpina L. Sp. pl. p. 67 (1753).

Var. parnassica Boiss. Fl. or. V, p. 605 (1884).

In Jer oberen Region des Chelmos. Höhe $1800 \mathrm{~m}$. 
332. Poa Timoleontis Heldr. in Boiss. Fl. or. V, p.605 (1884).

In cler Tannenregion des Chelmos. Höhe $1200 \mathrm{~m}$.

333. Gaudinia fragilis 1.. Sp. pl. p. 80 sub Avena (175.3); P. (le Beauv. Agrost. p. 95 (1812).

Bei Patras.

334. Lolium temulentum 1. Sp. pl. p. $8: 3$ (1753).

Unter Getreicle bei Kalarryta. Höhe $700 \mathrm{~m}$.

\section{CONIFERAE JUSS.}

335. Pinus nigra Arn. Kenise nach Mariazell, p. 8 (1785).

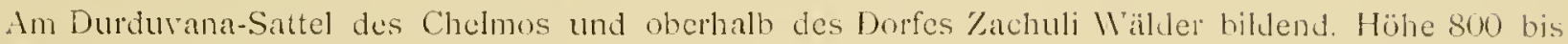
$1200 \mathrm{~m}$

\section{GNETACEAE Blum.}

336. Ephedra campylopoda C. A. Mley. in Bull. Pétersb. V. p. 34 (1847).

Bei Negaspilaeon. Höhe $700 \mathrm{~m}$.

\section{EQUISETACEAE DC:}

337. Equisetum palustre 1. Sp. pl. p. 1061 (175:3).

Sumpfiviesen bei lialarryta. Höhe $700 m$.

\section{Moose.}

338. Eucladium verticillatum L. Sp. pl. p. 1120 sub Bryo (1753); Brynl. europ. I, t. 40.

Auf Kalksinter bei Patras, steril.

339. Leptotrichum flexicaule Schleich. Plant. crypt. helv. Cent. 4, Nr. 9 sub Didymodonte (1807); Hampe in Linnaea XX, p. $7 \pm$ (1847). - Cymodonlimm flericamle Schwägr. Suppl. 1, 1, p. 115, t. 20 (1811).

Auf dem Chelmos, steril.

340. Didymodon rubellus Hoffm. Deutschl. Fl. II, p. 33 sub Bryo (1796: Bryol. europ. II, t. 1S.) (1846).

Auf dem Chelmos, steril.

341. Didymodon luridus Hornseh. in Spreng. Syst. JV, 1, p. 173 (1827); Bryol. ellop. 1J, t. 1S6 $(18+6)$.

Auf dem Chelmos, steril.

342. Trichostomum crispulum Bruch in Flora X11, 2, p. 395, t. 1, f. 4 (1829).

Bei Patras.

343. Trichostomum nitidum Lindb. Om de europ. Trichostom. p. 45, sub Tortula (1S64); Schinp. Syl]. ed. 2, p. $579(1876)$.

Bei Patras.

344. Barbula tortuosa L. Sp. pl. p. 1119 sub Bryo (1753); W" b. et Mor. Bot. Tasehenb. p.205 (1807). ln der Tammenregion des Olenos, steril.

345. Barbula cylindrica Tayl. in Mack. Fl. hib. II, p. 26 sub Zygotydria (1836); Schimp. in Hedwigia 1873, p. 47 et Syn. ed. 2, p. 208 (1876).

In der Tannenregion des Olenos, steril.

346. Barbula revoluta Schrad. Syst. Samml. Kiypt. Gew. 1, Ni. 54 sub Tor/mla (1796); Brid. in Schrad. Journ. I11, 2, p. 299 (1801).

Auf dem Chelmos mit Didymodon rubcllus, steril.

1 Liarbeitet von J. Breialler. 
347. Barbula muralis L. Sp. pl. p. 1117 sub Bryo (1753); Timm. Fl. Mlegalop. Prodr. p. 240 (1788).

Bei Patras, c. fruct.

348. Barbula inermis Brid. Bryol. univ. I, p. 581 pro var. \% Syutrichiac subulatae (1826); C. Nüll, Syn. 1, p. 624 (1849).

Auf dem Olenos und Chelmos, c. fruct.

349. Barbula intermedia Brid. Bryol. univ. I, p. 586 sub Syntrichic (1826); Milde Bryol. siles. p. 129 (1869).

In der obcren Region des Panachaicon mit Ortlotrichum cuptulatum, steril; auf dem Kastro bei Kalavryta, c. fruct.

350. Barbula ruralis L. Sp. pl. p. 1116 sub Bryo (1753); Hedw. Fund. II, p. 92 (1782).

In der Tannenregion des Olenos, c. fruct. und auf dem Panachaicon, steril.

351. Grimmia apocarpa L. Sp. pl. p. 1115 sub Bryo (1753); Hedw. Descr. I, p. 104. t. 39 (1787).

Auf dem Chelmos und Olenos, c. fruct.

352. Grimmia pulvinata L. Sp. pl. p. 1120 sub Bryo (1753); Sm. Engl. Bot. t. 1728 (1807).

Auf dem Kastro bei Kalarryta und auf dem Chelmos, c. fruct.

353. Grimmia trichophylla Grev. Scott. crypt. Fl. Nr. 20, t. 100 et Fl. Edinb. p. 235 (1824).

In der Tannenregion des Olenos spärlich und steril zwischen Fartula ruralis.

354. Zygodon viridissimus Dicks. Fasc. pl. crypt. IV, p. 9, t. 10, f. 18 sub Bryo (1801); Brown in Trans. of Linn. soc. Xill, 1, p. 575 (1819).

Aul dem Olenos zwischen Homalothecium sericcum, steril.

355. Orthotrichum cupulatum Hoffm. Deutschl. Fl. Il, p. 26 (1706).

In der oberen Region des Panachaicon.

356 Funaria hygrometrica L. Sp. pl. p. 1110 sub Mnio (1753); Sibth. Fl. Oxon. p. 285 (1794).

Bei Megaspilacon, reichlich fructificirend.

357. Bryum alpinum Huds. Fl. angl., p. 415 (1762).

Auf dem Panachaicon, steril. ?

358. Bryum capillare L., Sp. pl. p. 1121 (1753).

Var. cavifolium. Planta humilis dense caespitosa. Folia breviora, obovata et subpathulata, valde concava, latiuscule limbata, margine anguste recurvo, apicem versus plano minute serrato, costa in apiculum flexuosum excurrente, in sicco sinistram versus contoryuata. Capsula in pedicello breviore minor, cum collo et operculo $2 \cdot 5-3$ mm longus.

Auf Felsen und steinigem Boden auf dem Olenos.

359. Bryum bimoideum de Not. Epil. p. 383 (1869).

In der oberen Region der kyllene.

Bryum bimoidcum de Not. ist eine noch wenig bekannte, nicht aufgeklärte Art. Der Autor vergleicht sic mit Brytum pallescens, Br. psendotriquetrum und Br. bimmm. Zum Schlusse sagt er: "Num varictas Bryi bimi dioica? - Venturi und Bottini stellen dieselbe in "Enumerazione critica dei Muschi italiani «, p. 34 (1884) mit einem? als Synonym zu Br. cuspidatum Schimp. - Limpricht in Rabenh. Krypt. Fl. IV. Band, Il. Abth., p. 442 (1893), vermuthet darin eine kleinere Form von Br. pseudotriquctrum.

Die hier angeführte Pflanze von der Kyllene, die nur junge Fruchstiele ohne Kapseln trägt, ist habituell dem Br. cuspidatum oder dichtrasigen Formen des Br. bimum ähulich, letzterem gleicht sie auch in den Blättern, unterscheidet sich jedoch von demselben durch den diöcischen Blüthenstand.

360. Philonotis laxa Limpr. in Rabenh. Kirypt. Fl. IV, 2, p. 563 (1893).

Auf nassen lielsen bei Megaspilacon, steril. 
361. Philonotis calcarea Schimp. Coroll. p.86 (1856). - Barmama calcarat Bryol. europ. IV, t.325 $(1886)$.

Aul dem Chelmos und Panachaicon, steril.

362. Leptodon Smithii Dicks. Fasc. plant. crypt. II, p. 10, t. 5, 1. 4 sub Hypno (1790); Moln ()bs. bot. p. 27 (1803).

Auf dem Olenos, spärlich zwischen Homalothecinm sericenn; c. fruct.

363. Leucodon morensis Schwïgr. Suppl. II, I, p. 82, t. 125 (1823). - IIInmmm morense Schleich. in Sched. - Lcucolon scinroides P. mormsis De Not. Sillab. musc. p. 79 (1838).

In der 'Tamnenregion des Olenos, reich fruchtend.

304. Isothecium myurum Brid. Bryol. univ. II, p. 367 (1827). - Hypmmm mummm Poll. Hist. pl. Palat. III, Nr. 1054, f. 8 (1877).

In der Tannentegion des Olenos, mit Homalothecinm sericenm steril.

365. Isothecium viviparum Neck. Delic. gallo-belg. II, p. 475 sub Hypno (1768); Lindb. Rev. crit. icon. 11. Dan. in Actil soc. scient. fenn. X, p. 12. (1870). - I. mymmm Brid. Bryol. univ. II, p. 367 (1827) In der 'Tamnenregion des Olenos, mit Homalohlecinm sericcum, steril.

366. Homalothecium sericeum L. Sp. pl. p. 1127 sub Hyllo (1753); Bryol. europ. V, t. 456.

An Baumstämmen in der Tamnenregion des Olenos, c. fruct. und des Panachaicon, steril.

367. Scleropodium illecebrum Schwägr. Suppl. I, II, p.225 sub Hypno (1816); Bryol.europ. VI, t. is.57. In der Tannemegion des Olenos, steril.

368. Hypnum falcatum Brid. Musc. Recent. II, II, p. 63, t. I, f. 6 (I801).

Auf dem Chelmos, mit Philonotis calcarea, steril.

369. Hypnum cupressiforme I. Sp. pl. p. 1129 (1753).

In der Timnenregion des Olenos, mit Homalotlecimm sericamm, steril.

37.0. Radula complanata Dum. Recueil d'obs. p. 14 (1835).

Auf Baumrinden in der Tannenregion des Olenos, c. 1 . $q$ et $\sigma^{7}$.

371. Madotheca rivularis Nees Naturg. 11I, p. 196 (1838).

In der Tannenregion des Panachaicon, steril.

372. Frullania dilatata L. Sp. pl. p. 1133 sub Jungermamia (1753); Dum. Recueil d'obs. P. I3 (1835). In der Tannenregion des Olenus, c. perianth.

\section{Flechten. ${ }^{1}$}

Die Flechten stammen von Patras, Kalavryta und vom Chelmos, Olenos, von der Kyllene und dem Panachaicon. Die Substrate sind Kalke und Kalkconglomerate, Kalk mit Quarz, hornsteinartige ${ }^{2}$ und opalartige Kieselausscheidungen, ausserdem ein Ziegelstück von Patras und ein Stück Sandstein vom Chelmos, Rindenstücke von Olea, Jugluns, Ailanlhus und Platanus von Patras, Fichtenrinde vom Olenos, Kiefernrinde vom Chelmos, Erde von Patras und dem Koloster Megaspilaeon. Die auf diesen Substraten gefundenen lilechten sind im Nachfolgenden aufgezühit.

373. Collema furfum Ach. Prodr. p. 132. - Ny1. Syn. 1, p. 107.

Steril auf Kalk vom Olenos.

374. Collema cristatum Nyl. Syn. 1, p. 109. - Linn. Spec. pl. p. 1610 sub Lichene.

Steril auf Kalk von Kalavryta.

1 Bearbeitet von J. Steiner.

2 Das in Bd. LXI, p. 252 der Denkschriften irrthümlich als Feldspath rom Peristeri angefühte Mineral ist ebenfalls ein eigenthümlicher hornsteinartiger ?uarz. 
275. Synechoblastus nigrescens Trevis. Caratt. Collem. 1853. - Arn. Jur. Separ. p. 279. - Huds. Fl. Angl. p. 450.

Steril auf Fichten rom Olenos, auf Pinus-Rinde rom Chelmos.

276. Usnea ceratina A ch. Univ. p. 619.

Steril auf Fichten vom Olenos.

377. Evernia prunastri Ach. Univ. p. 442 (exi. 5). - Lin11. Spec. Pl. p. 1147 sub Lichenc.

Olenos.

378. Sticta linita Ach. Syn. p. $234-$ Nyl. Syn. J, p. 353.

Steril häutig an Fichtenıinde vom Olenos.

379. Peltigera canina Nyl. Syn. 1, p. 324. - Li nn. Fl. Suec. p. 324.

Steril auf moosiger Rinde vom Olenos.

380. Parmelia acetabulum Dub. Bot. Gall. II, p. 601. - Neck. Delic. p. 506 sub Lichenc.

Thallus subtus mox rufofuscus. KHO sanguineo rubet. Pycnides numerosae mox tuberculiforme emersae nigrae. Arthrosterigmata ramosa. Pycnosporae rectae $4.5-7$ \% 1 g., 0.5 थ. 1 t.

Melrere Exemplare, sterile und mit Apothecien auf Fichtenrinde vom Olenos.

381. Physcia (Sect. Anaptychia) ciliaris DC. Fl. fi. IJ, p. 396. - I inn. Spec. plant. p. 114t sub Lichcnc. Steril zwischen und über Sticta linita rom Olenos.

382. Physcia pulverulenta Schreb. f. venusta A ch. Meth. F. 211 sub Parmiclia.

Auf Rinden rom Olenos.

383. Physcia stellaris Nyl. Prodr. p. 307. - Linn. Sp. plant. p. 1144 sub Lichcne.

Forma adpressa quaedam optime evoluta. KHOthallus extus intusque non mutatur v. sero levissime tantum lustescit. Apothecia et sporae speciei. Pycnides atrae mox tuberculiforme emersae. Arthrosterigmata ramosa, sterilia elongata supra incrassata saepe immixta, pycnosporae $2-3.5 \% \lg .0 .5-0.7 \% 1$.

Auf Juglans, Ailanthus und Plantanms von Patras. Im Herb. Eggerth (Univers. Wien) befindet sich bei Pl. dimiliata (rergl. Arn. Fl. 1887, p. 1445) ein nicht näluer bezeichnetes Exemplar einer stellaris auf Oelbaumrinde von Corfu, welches der Flechte von Patras nahe entspricht.

Die f. leptalca Th. Fr. Sc. p. 140. - Ach. Prodr. p. 108 sub Lich. auf Pinus-Rinde vom Chelmos.

384. Xanthoria parietina Th. Fr. Arct. p. 67. - Linn. Sp. pl. p. 1143.

Auf Pinus-Rinde vom Chelmos, auf Olea, Ailanthus von Patras. Die f. aurcola Ach. Univ. p. 47s auf Kalk von Kalaviyta. F. imbricata Mass. exs. 32 auf Ailautlus.

385. Caloplaca (Sect. Amphitonia) elegans Th. Fr. Scand. p. 168. - Link. Ann. d. Bot. I, P. 37 sub Lich.

Nur einige Thalluslappen auf Kalk vom Chelmos.

386. Caloplaca (Sect. Amphiloma) callopisma Th. Fr. Sc. p. 169 - Ach. Unir. p. 437 sub Lecan. Aul Kalk von Patras und Kalavryta. F. centrolenca Mass. von Patras.

387. Caloplaca (Sect. Apliloma) aurantia f. centrifuga Mass. in Sched. 1855 p. 66 exsicc. n. 94.

Ein kleines Exemplar auf Kalk von kalavryta, welches am besten Mlass. exs. 94 dext. entspricht.

388. Caloplaca (Sect. Amphilomı) granulosa Mïill. Arg. Princ. p. 40 sub Amplitl. - Arn. Jura Scp. sub Plyyscia.

Der Thallus noch spärlicher entwickelt, als ihn das untere Exemplar in Hepp cxs. 908 zeigt.

Neben Cal. callopisma auf kalk von Patras.

389. Caloplaca aurantiaca Th. Fr. Sc. p. 177 - Lightf. Fl. Scot. Il, p. 810 sub Lich.

Auf Sandstein rom Chelmos. Die f. placidia Mass. Symm. p. 32 auf Kalli von Kalaryta.

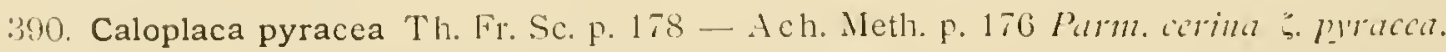

Auf Olea von Patias und auf Pinns-Rinde rom Chelmos. 


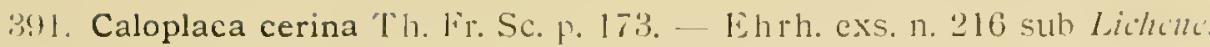

Dic f. Ehrhati auf Pinns-kinde rom Chelmos.

392. Caloplaca (Sect. Pyronodesmia) chalybaea T'h. Fr. Sc. p. 17'2. - Duf. in E. 1'r. Lich. Eur. p. 125 sub Parmelia.

Die nomale Pllanze auf halk von kalavryta und dem Panachaicon.

393. Caloplaca (Sect. Pyrenodesmia) variabilis Th. Fr. Sc. p. 172. - Pers. Ust. Ann. 1794, p. 26 sulh Lichenc.

Die normale Pflanze auf Kalk von Kalavryta und rom Panachaicon. Eine auffallende Form, allerdings in sehr kleinen Exemplaren, neben Cal. callopisma auf kalk von Patras:

Thallus areolatus albescens, discus apotheciorum albo-pruinosus, margo albofarinosus, involutus, primum saltem radiatim striatus, crassus. Die Pflanze entspricht einem Exemplare aus dem Nachlasse von Loylia im Herb. des k. k. Hofmuseums in Wien, welches als Lecan. Tariabilis f. dealbata Ny 1.13 .1 ine dit. bezeichnet ist. Ein sehr spärliches Exemplar findet sich auch auf einem Stücke Kalk, welches Herr Oberstlieutenant Hartl auf dem Tringia sammelte. Vielleicht ist es dieselbe Form, ron der Th. Fr. Sc. p. 173 sagt, dass sie der Lecan. Agardhiana Ach. beigemischt sei. Thre Apothecien sind äusserlich denen der Lecan. Agardhiana Ach. sehr ähnlich.

394. Caloplaca (Sect. Pyrenodesmia) fulva Müll. Arg. Fl. 1872, p.470. - Anzi Symb. p. 7 sub Ziora Exs. n. 393.

Auf Kalk vom Olenos und rom Panachaicon.

305. Caloplaca (Sect. Pyrenodesmia) intercedens Stnr. Sitzh. d. li. Ak. d. Wiss. Wien, math.-1naturw: C1. Bd. C11, Abth. I, p. 163. - Trevis. Lich. Venet. n. 33 sub Pyronodesmia.

Die normale Pflanze auf Kalk von Kalarryta.

Var. albomarginata Stnr. Denkschr. d. k. Akad. d. Wiss. Wien, Bd. LII, p. 263. Vom Panachaicun.

Hicr auch eine Form, welche wegen der zuerst deutlich eingesenkten, dann rortretenden Apothecien (0.7 $\mathrm{mm}$ diam. oder kleiner) und ihrer thallodischen, weissen und dicken Berandung zu albomarginala gehört, deren Thallus aber den Kalk violettgrau färbt, und deren Discus schon trocken, mehr oder weniger dunkel grauviolett elscheint, benetzt aber braunlichgrau oder violettgrau wird. Sie kann als f. cinereovinosu m. der var. albomarginala untergeordnet werden.

Das freundliche Entgegenkommen des Herm Appellgerichtsrathes Dr. Arnold, für das ich hier meinen Dank ausspreche, hat es mir möglich gemacht, in die von ihm und von Körber unterschielenen Formen durch die Originalexemplare Einsicht zu erhalten. Darnach unterscheidet sich allomarginata m. von alpina Hepp-Arn. Verh. d. z.b. G. Wien 1869, p.640 (die alpina Arn. Verh. 1879, p. 376, sowie die nigricans Arn. V'erh, 1869, p. 640 und gramulosa Arn. ibid. sind nach ihrem Thallus Formen der Cal. arabilis) durch die zuerst eingesenkten Apothecien, verhält sich also zu alpina so, wie die Hauptform zu frandulentak k.

396. Caloplaca (Sect. Blastemia) percrocata Stnr. - Aln. Lich. Exs. $1859-1893$. p. 26 und p. 29 sub blastenia. - Arn. Exs. 924 Blast. arenaria var. pererocita.

Auf Hornstein rom Panachaicon.

39\%. Gyalolechia aurella Arn. Jur. Sep. p. 92. - Hoffm. D. F1. 197 sub I'orrucuria.

Häufig vom Chelmos, Olenos, von Kalarryta und vom Panachaicon, theils über andere Flechten, theils auf dem Gestein zerstreut.

398. Gyalolechia lactea Aln. FJ. 1881, p. 311 et exs. Monoc. n. 95. - Nass. in Sched. 1856, p. 133 und exs. 236.

Auf lialk mit Hornstein von Patras.

Die Sporen ron Mlass. exs. '236 (Herb. Univers. Vienn.) sind abgerundet tönnchenförmig, mit dicker Scheidewand $12-1+\mu$ (selten $16 \mu$ ) lg., $75-9 \mu$ lt., entsprechen also denen der Gattung nicht. Arn. exs. 


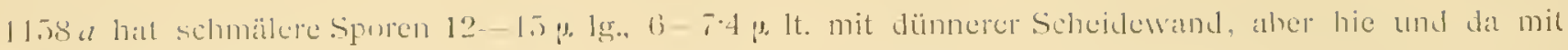
Mittelporus.

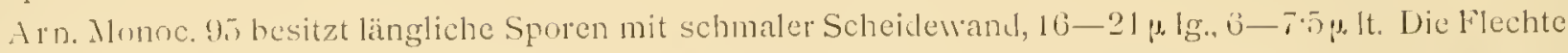
von Patras, in der Tracht der Apothecien der Münchener gleichend, hat längliche Sporen mit durchaus

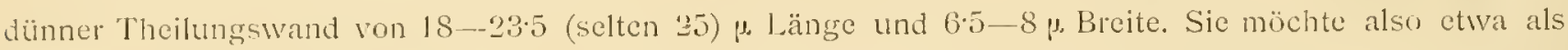
f. macrospora zu lacter (Mass.) Arn. gestellt werden, rorausgesetzt, dass lactea Mass. anderer Herb. zu laclea Arn. stimmt.

399. Gyalolechia (Sect. Candelaria) vitellina Th. Fr. Spitz. p. 19. - Ehrh. exs. n. 155 sub Lichene.

Vom Chelmos, Patras, dem Panachaicon und Kyllene vorliegend, sowohl auf dem Gestein als auch über verschiedenen filechtenkiusten.

100. Rinodina exigua Arn, Jur. n. 170. Ach. Prodr. p. 69 sub Lichene.

Auf Pinns-Rinde vom Chemos.

401. Rinodina corticola A r1. Verh. l. zoul. bot. Ges. Wien 1879, p. 370. - Arn. ibid. 1868, p. 952. sub Rinol. teichoph. var. - Anzi exs. 377 Rin. metabolica var.

Thallus et margo apotheciorum cinereus. Sporae $18-23.5 \mu \mathrm{lg}$., $9-12 \mu \mathrm{lt}$. Sporoblast. subcuadratis,

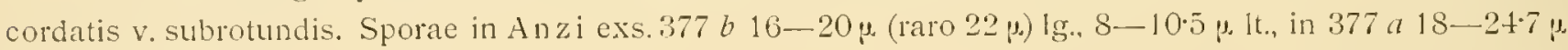
$\lg ., 10-14 \mu_{.} 1 \mathrm{t}$.

Auf Pinns-Rinde vom Chelmos. Die Pflanze gleicht im Habitus der Rin. Travisani Hepp. exs. So.

402. Rinodina Bischoffii Hepp. Lich. Eur. exs. 11. 81.

Auf kalk rom Panachaicon. Auf Kalk vom Chelnos sehr spärlich, eine auffallende Form mit ockerig bestäubten Areolen.

403. Rinodina immersa Arn. Jur. Sepr. p. 104. -- Krb. Par. p. 75 sub Rin. Bischoffii P. immersa.

Auf mehreren kalkstücken vom Panachaicon.

404. Rinodina ocellata Th. Fr. Sc. P. 204 Obs. - Hoffm. Pl. lich. 92 sub Ierrnc. videlur - Ach. Prodr. p. 61 sub lich.

Die normale Pflanze mit braungrauem Thallus auf Kalliconglomerat von Kialavryta.

405. Acarospora smaragdula Arn. Jur. Sep. p. 101. - Walilb. in Ach. Meth. Suppl p. 29 sub Endocarp.

Auf Hornstein vom Panachaicon, vorherschend reihenweise geordnete Areolen in den Ritzen des Gesteines. Die Farbe der Areolen gleicht der von A. l'crmonensis $\mathrm{Ml}$ as S. auffallend, die Form ist aber die von rufescins. Sporae $2-4 \mu .1 \mathrm{~g} ., 1.5-2 \mu 1 \mathrm{t}$.

406. Acarospora fuscata Arn. Jur. Sep. p. 101. - Schrad. Spic. p. 8?.

Sehr spärlich vom Panachaicon. Sporae $3.5-6.5 \mu . \lg ., 1 \cdot 8-2.5 \mu .1 t$.

407. Lecania (Sect. Dimerospora) Rabenhorstii Arn. Jur. Sep. p. 124. - Hepp. Eur. p. 75.

Auf Kalk vol Patras.

408. Lecanora (Sect. Placodimm) crassa A c1. Univ. p. 413. - Huds. Fl. Angl. 11, p. 530 sub Lichonc. Auf Kalkconglomerat von Kalaryta. Die f. caespitosa Vill. Dauph. p. 976, t.55 auf Erde beim Ǩloster Megaspilaeon bei Chelmos.

109. Lecanora (Sect. Placodimm) muralis Schär. Enum. 4 higg. ¿2 (sec. Nyl. Scand. p. 1:33). - A rn. Jur. Sep. 97 sub Placod. - Schrcb. Spic. p. 130.

Vorherrschend var. diffracta Ach. Prodr. p.63. - Nyl. Scand. p. 133, rom Chelmos, Olenos und dem Panachaicon. Von der Kýllene liegt dic form mit vollständig krustenförmigem, am Rande nicht effiguriertem Lager und eingesenkten, aus dem Lager nicht vortretenden Apothecien vor. Vollständig übereinstimmende Exemplare sammelte Unger neben der gewöhnlichen diffracla auf dem St. Croce auf Cypern (Herb. d. k. k. Hofmus. Wien, sub Plac. sax. Var. diffractuml). Vielleicht entspricht sie der mir unbekanuten f. areolala Leigth. in Herb. Hepp (comp. Stitzenb. Helv. p. 88). 
Ich bin der. Mesnung dass diese form ron diffirata Ach. unterschieden werden sollte und nach ver Beschreibung l. c. auch getrennt werden kann.

Dic var. virsiculor Ach. vom Panachaicon.

410. Lecanora (Scct. Macodium) subcircinata Nyl. Fl. 1873, p. 18.

Thallus KHO rubescit. Pycnides majores, compositae, tandem emergentes thallo obvallatac, supra atrac. Stcrigmata fertilia simplicia v. subsimplicia ramosa, sterigmata sterilia septata, supra sacpe incrassata, immixta. Pycnosporae rectae 35-7 p. lg., 1-1.8 \%. 1t. Auf homsteinartigem Quarz von Olenos und dem Panachaicon.

411. Lecanora (Scct. Placodium) albescens Arn. Jur. Sep. p. 114. - Th. Fr. Sc. p. 252 p.p. - Hoffm. D. Fl. 11, p. 165 sub Psora.

Thallus suborbicularis ambitu cffiguratus.

Auf Kalk von Patras.

412. Lecanora atra Ach. Univ. p. 344 p. p. - Huds. Fl. Angl. p. 530 sub Lichene.

Pycnides supra coeruleo virides. Pycnosporae ad $18 \mu \mathrm{gg}$. Auf Kalk mit Hornstein vom Olenos und Panachaicon.

413. Lecanora subfusca Ach. Univ. p. 393. - Lin 11. Suec. p. 409 sub Lichene.

Var. pinastri Schär. Spic. p. 390 auf Pinus-Rinde vom Chelmos. Var. rugosa Pers. in Ach. Univ. p. 304 auf Ölbaumrinde von Patras. Eine kleinfrüchtige Form, die der glabrata Ach. Univ. p. 393 nahc steht, auf Ficus-Rinde von Patras.

414. Lecanora Hageni Ach. Univ. p. 367 p. p. - Ach. Prodr. p. 57 sub Lichene.

Die Var. umbrina Ehrh. mit unbereiften Apothecien auf Pinus-Rinde vom Chelmos.

415. Lecanora angulosa Ach. Univ. p. 364. - Schreb. Spic. p. 136 sub Lichone.

Discus apotheciorum $\mathrm{CaCl}_{2} \mathrm{O}_{2}$ citrinus. Auf Fichtenrinde vom Olenos.

416. Lecanora pallida Arn. Jur. Sep. p. 112. - Schreb. Spic. p. 133.

Discus apothecium $\mathrm{CaCl}_{2} \mathrm{O}_{2}$ non mutatur. Auf der Rinde von Pimus-Zweigen vom Chelmos.

417. Lecanora sordida Th. Fr. Arct. p. 115 p. p. Th. Fr. Scand. p. 246. - Pers. Ust. Ann. VIIJ, p. 26.

Auf Hornstein vom Chelmos. Daselbst auch die var. bicinclu Th. Fr. Sc. p. 246. Pycnides inmersae, atrae. Sterigmata ramosa subsimplicia v. simplicia.

Pycnosporac falcatae, arcuatae v. flexuosac $18-25 \mu \mathrm{gg}, 0.5 \mu \mathrm{kt}$.

418. Lecanora polytropa Nyl. Sc. p. 164. - Ehrh. exs.n. 294 sub Lichene.

Auf Hornstcin vom Chelmos und Panachaicon.

419. Lecanora dispersa Flk. D. Fl. IlI, p. 4. - Pers. in Ust. Ann. VIl, p. 27 sub Lichcne.

Apothecien auf dem Gestein und über Flechtenkrusten von Patras, Kalavryta und von der Kyllenc.

420. Lecanora crenulata Arn. Jur. Sep. P. 115. - Dicks. Crypt. 3, p. 14. - Syn.: Lecan. caesioalba lirb. Par, p. 82.

Discus fuscescens v. obscure lividus plus minus pruinosus, margo tumidulus demum crenatus.

Auf Kalk vom Panachaicon. Nicht selten auch die f. macta Sommerf. Comp. Th. Fr. Sc. p. 253.

421. Lecanora Agardhiana Ach. Syn. p. 152. - Syn. Lccan. Agurdhianoides Mlass. Ric. p. 11.

Auf Kalk des Panachaicon. Epithecium $\mathrm{KH} H \mathrm{H}$ non mutatum, $\mathrm{HNO}_{3}$ adh. rubescit. Sporae $7-11 \mu \mathrm{lg}$., $4 \cdot \bar{\tau}-6 \mu$ 1t. Pycnidcs supra coeruleo-virides. Sterigmata simplicia breviora. Pycnosporae arcuatae $\checkmark$. varie flexuosae $14-16.5 \mu$ ig., $0.7-1 \mu .1 \mathrm{t}$.

422. Lecanora (Sect. Aspicitia) calcarea Sommerf. var. concrelı Schär. Spic. p. 73.

Auf kalk von Kalarryta und dem Panachaicon.

Die var. viridescons Krb. Par. p. 95. - Mlass. Ric. p. 40 häufig auf Jaalk vom Panachaicon, ein Exemplar, auch der Tracht nach, 11 ass. exs. 263 vollständig entsprechend. Sterigmata simplicia v. subsimplicia 
lamosil, sterigmata sterilia. supra incrassata, silepius immixta. Pycnusprate rectae v. raro levissime curvala 7-10 p. Ig. Dic f. ochraca Anzi mit grimlichen mehr oder weniger ockerig bestäubten Areolen häulig lon Olenos, Patrats und Kalavryta. Alle formen rerschiedene Syntrophen bcherbergend.

422. Lecanora (Sect Aspicilia) farinosa Nyl. Fl. 1878, p. 248. - Flk. in Berl. Mag. 1810, p. 125.

Auf lialk rom Panachaicon die normale l’flanze.

423. Lecanora (Sect. Aspicilia) trachytica Arn. F1. 1887, p. 150. - Mass. Ric. F. 44.

Thallus kHO extus intusque optime sanguineo rubet, $\mathrm{CaCl}_{2} \mathrm{O}_{2}$ et 3 non mutatur.

Discus apotheciorum madefactus fuscescit $v$. non mutatur. Pycnides immersac supra atrae varie rufofuscac. Stergmata fertilia simplicia v. subsimplicia ramosa. Sterigmata sterilia filiformia, crassiora, scptata saepius immixta.

Pycnosporae bacillares rectae $v$ raro leviter curvulae $5-10 \mu .1 \mathrm{~g} .1-1 \cdot 8 \mu \mathrm{lt}$.

Vom Panachaicon und von der Kyllene; von ersterem in mehrereren Exemplaren.

Die Apothecien und Pycniden behalten, wenn sie benetzt werden, hine schwarze Farhe oder verändern sie, und zwar an denselben Exemplaren, in Braun, ohne dass dabei das Alter von einem Einfluss wäre, ganz so, wie es auch bei $M$ ass. exs. 260 der Fall ist.

Nachdem ich nun trachytica Mass, in gut entwickelten Exemplaren von verschiedenen Standorten kennen gelernt, bin ich sicher, dass die in Sitzungsb. d. k. Ak. d. Wriss. Wien, math.-nat. C1. Bd. Cll, Abth. 1, p. 165 als fragliche trachytica Mass. vom Hymettus angeführte Flechte nicht zul dieser Art gehört.

In ihrer auffallenden Reaction mit kHO, welche darin besteht, dass nur die Markschichte roth wird, während die Rindenschichte ungefübt bleibt, und in der Tracht der Arcolcn mit Ausnahme der Randareolen stimmt sie ganz mit Pacle. calcarea f. bullosa Mass. exs. 266 übercin. Dagegen hat die bullosa punktförmig geöfnete Apothecien, während sic bei der Flechte vom Hymettus einen deutlich erweiterten Discus bilden.

424. Lecanora (Sect. Aspicilia) olivacea Bagl. e Car. in Comm. Crit. Ital. 1, p. 441 (1864).

Areolae primum subrotundae tandem mutua pressione angulosae, cervino-fuscae albo marginatic tandem nigrescentes, hic inde cincrascentes, opacae, prothallo ohscuro vestitac, madefactae olivaceae ad $0.8 m m$ diam. v. minores. Apothecia immersa. parva, tandem subrotunda disco plus minus obscure sanguineo rufo, saepe linea alba thalli decorticati marginata. Excipulum mere thallodes, stratum gonidiale sub hypothecio crassum. Paraphyses crassiores, septatae, supra plus minus incrassatae et rufo-fuscae.

Sporae raro evolutae $12-14 \mu, \mathrm{lg}, 7-9.5 \mu .1 \mathrm{t}$. Thallus $\mathrm{kHO}$ v. Ca $\mathrm{Cl}_{2} \mathrm{O}_{2}$ non mutatus, J leviter violascit.

Auf Kalk mit Hornstein von der Kyllene. Die Flechte stimmt mit der Beschreibung und Abbildung in Bagl. Car. Anacr. 'dei Lich. della Valsesia 1880 . p. 225 und tab. 11, Fig. 24 und cinem Originalexemplar im Herb. Eggerth (Univers. Wien).

Aspic. olizacea f. cerrinocuprea Arn. in Verh. d. zool.-bot. Ges. Wien 1876, p. 357 und ibiu. 1879 p. 381. cxs. 754; in Arn. Lich. cxicc. 1859-1863 mit cuprocatra Nyl. vereinigt, unterscheidet sich durch etwas kleincre. besonders aber dickere öfter grauliche Areolen und etwas mchr vortretende Apothecien.

Lecan. cupre-atra Ny1. Fl. 1866, p.417, steht der olizacea noch näher. Arn. exs. 1114. Loyka 44 und ZW. 715 erschienen von der normalen olizaca nur dadurch etwas verschieden, dass der dunkle Prothallus vorherscht, die noch dünnen Areolen öfter inselartig auf ihm erscheinen und die Apothecien sehr klein und meistens heller gefürbt sind. Vergl. Arn. Verh. d. zool.-bot. Ges. Wien 1893, p. 405.

Es liegt aber bei Zw. 715 (Herb. Univ. Vienn). ein Exemplat, Welches von olizaca Bagl. Wohl kaum irgendwic zu unterscheiden wäre. Der innere Bau der Apothecien ist überall übereinstimmend, die Sporenbildung nur bei Arn, 754 eine reiche. Was die Reaction der Markhyphen gegen J anlangt, ist ein Unterschied nur in Bezug auf deren Intensitä vorhanden. Die Hyphen der Gurgler Flechte Arn. 754 färben sich schwarzviolett, wïhrend sonst eine leichte, aher deutliche und gleichmilssige Fürbung eintritt. Je dicker dic Markschichte ist, umso stärker tritt die Reaction ein. Pycniden fand ich nur bei Arn. 754. 
Pycnides tanden tuberculiformes, emersae atrac, sub micr. olivaceo-virides. Sterigmatil $14-16 \mu$ gg. simplicia r: subsimplicia, ramosa, tenuia. Sterigmata sterilia crassa, septatia supra clavata.

Pycnosporae rectae $4.5-7 \mu . \lg ., 05-1.8 \mu_{0} \mathrm{it}$.

426. Lecanora (Sect. Aspicilia) cinereorufescens Th. Fr. Sc. p. 284 (Arct. p. 134 p. p.). - Ach. Univ. p. 677 sub trecolaria.

Thallus cinereo-plumbeus, plus minus ochraceo-suffusus. KHO adh. lutescit, $\mathrm{CaCl}_{2} \mathrm{O}_{2}$ non mutatur.

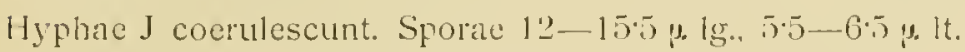

Zwei kleine Exemplare auf Kalkhornstein vom Chelmos, die nach der Fürbung des Lagers als f. ochraced Krh. Syst. p. 162 bezeichnet werden können.

427. Lecanora (Sect. Aspicilia) Prevostii Th. Fr. Sc. p. 288. - Var. affinis 1]ass. Symm. p. 23 et exs. 330 .

Ein kleines Exemplar auf Kalk rom Panachaicon, welches zwar del typischen affinis Mass. nicht voltständig entspricht, weil die Apothecien etwas grösser und mehr gerundet sind, daher auch mehr vortreten, aber dieser doch näher als der normalen Prevostii steht

Pycnides apotheciis jurenilibus similia. Sterigmata simplicia ramosa, pycnosporae bacillares, rectae

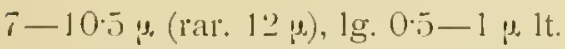

\section{Diploschistes violarius Nyl. - f. graccus Stnr.}

Thallus crassus, verrucoso bullatus caesius v. plumbeo cinereus, madefactus viride cinereus, ad marginem extenuatus albidus. Apothecia hic inde gyrose congesta iis scruposae simillima margine subcrenulato. Sporae magnae, multo-septatae, utroque apice acutatae non raro ad $35 \mu$. lg. 21 p. It., membrana juniorum 1 coertlescit. Pycnides immersae. Sterigmata sterilia filiformia, septata, fertilia simplicia, ramosa. Pycnosporae breviter baculiformes rectae $3 . \overline{0}-6 \%$ Ig., $1-1.4 \% \mathrm{lt}$.

Thallus $\mathrm{KHO}$ lutescit, $\mathrm{Ca} \mathrm{Cl}_{2} \mathrm{O}_{2}$ intense violascit, J passim levius coerulescit. In Bezug auf die Reactionen stimmt die Flechte vollständig mit Arn. exs. 890 überein (vergl. dagegen Hue Add. und L amy d. C. Chap. Cat. p. 94, wo J - angegeben ist). Da violurius Ny., so weit ich zu finden vermag, nur durch die Farbenreactionen von scruposus verschieden ist, so dürfte diese Art. weitere zustimmende Funde vorausgesetzt, wohl nur als geographische Einheit aufzufassen sein, Woraus sich wieder ergiht, dass die Bezeichnung für die f. graecus nur eine provisorische sein kann.

429. Pertusaria communis DC. F1. fr. IJ p. 3:20.

Steril auf Fichtenrinde vom Olenos.

\section{Pertusaria subinquinata Stn $\mathrm{r}$.}

Thallus mediocris, areolatus, viride-cinercus, madefactus viridis, reag. solit. caeterum non mutatus, $\mathrm{KHO}$ add. $\mathrm{CaCl}_{2} \mathrm{O}_{2}$ lutescit. Areolac fertiles nunquam elatae. Apothecia 2-3 in quavis areola, primum subrotunda disciforme dilitata, deinde confluentia pseudodiscum non interruptum ad $1 \mathrm{~mm}$ latum, reliquiis areolae marginatum, formant.

Discus primum obscurus mox fuscus, madefactus dilute fuscus. Epithecium fuscescens KHO leviter violascit.

Sporae octonae late ellipticae apicibus attenuatis ad $33 \mu .1 \mathrm{~g} ., 20 \mu$. It. $\mathrm{v}$. minores, p. m. p. uniserialiter in ascis cylindricis. $J$ adls. asci tantum coerulescunt. Pycnides frustra quaesivi.

Ein ziemlich dürftiges Exemplar neben Rhiz. geographicum vom Panachaicon.

Die Flechte steht der inquinuta (Ach) Th. Fr. Sc. p. 311 nahe, weniger deren kleinfrüchtigen Formen personata Th. Fr. 1. c., chiodectonoides Bagl. Mass. Misc. p. 26 und nolens Nyl. Sie unterscheidet sich aber durch ihren Thaflus, die bald hell gefürbten grossen Apothecien und die fast immer einreihigen Sporen. Dic Sporen selbst zeigen in der ganzen Gruppe keine haltbaren Unterschiede.

431. Bilimbia coprodes Krbb. Par. p. 166. - Comp. Stitzenb. Lec. sabulet. p. 60 et Th. Fr. Sc. p. 385. Thallus inconspicuus granulosus, fuscus. 


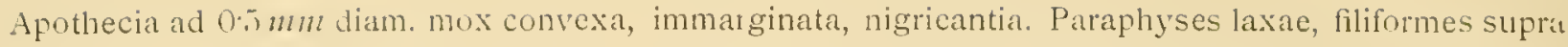
incrassatae et septatae epithecium obscure fusco-viride $r$. coeruleo-viride formant. Hypothecium rufo-fuscum v. violaceo-rufum. Sporae $16-20 \mu 1$ g., $3-4.5 \%$ it. 3-septatae, rectae v. leviter curvulae.

Nur cinige Apothecien auf Kalk von Patras.

432. Bilimbia episema A rn. Fl. 1874, p. 94. - Nyl. Prodr. p. 125 sub Lecidea.

Epithecium obscure fuscum $r$ atro-fumosum, excipulum atro-violaceum, hypothecium rufum v. violaceorufum, hymenium non raro subviolaceum. $\mathrm{kHO}$ hypothecium magis violascit. $\mathrm{HNO}_{2}$ epithecium et p. p. hymenium rubro-violascunt. Asci clavati ad $35-40 \mu 1$ g.. $12 \mu$ t., membrana supra incrassata. Sporae octonae elliptico-elongatae $9-14 \mu \lg ., 4-5.8 \mu .1$. 1 -septatae $v$. raro 3 -septatae.

Auf Lecan. calcarea von Patras. Die Flechte gleicht in ihrer Tracht und Wachstumweise sowohl als den inneren Fruchtmerkmalen Arn. exs. 1194 vollständig.

Die Sporen sind, genau wie dort, zweizellig, selten undeutlich, noch seltener deutlich vierzellig (vergl. dagegen Rabenh. Cryptog. Fl. Bd. 1, Abth. 111, p. 329). Der Gattungsname stützt sich allerdings nur auf die seltenen viertheiligen Sporen, im Übrigen bleibt es fraglich, ob die Verwandtschaft mit Patell. aspiciliae II üll. Arg. F1. 1872, p. 488 und Lecid. supermula N.yl. F1. 1876, p. 574 nicht ebenso gross ist, als die zu einer Bilimbia.

433. Lecidea (Sect. Psora) decipiens Ach. Meth. p. 80. - Ehrh. in Hedw. Stirp. Crypt. 11 (1819), p. 7.

Die normale Pflanze substeril auf Erde von Patras.

434. Lecidea (Sect Bialora) fuscorubens N yl. Bot. Not. p. 183.

Paraphyses crassiores, optime et saepe constricto-septatae, ramosae. Pycnides s. mic. rufofuscae.

Sterigmata tenuia, simplicia v. crassiora, septata (Arthroster.) sed cellula apicali tantum fertili.

Sterigmata sterilia, supra saepe incrassata, immixta. Pycnosporae breviter baculiformes rectae $4-5$ ». lg. $07-1 \mu .1$ t.

Auf Kalk vom Panachaicon.

435. Lecidea parasema Arn. Jur. Sep. p. 165. - Ach. Prodr. 1798, p. 64 p. p.

Auf Olea von Patras. Die f. ruğulosa Ach. Univ. p. 176 Syn: grandis Fiv. in Krb. Syst. p. 244 vorherrschend mit graulich ockerfarbigem Thallus, häufig auf Pinus-Rinde vom Chelmos. Pycniden und Pycnosporen normal.

436. Lecidea latypea Ach. Meth. Suppl. p. 10.

Vom Chelmos und Panachaicon. Hier wächst die Flechte auf dem 'T'hallus der Lccan. calcurea, hat aber ihre eigenen wohlausgebildeten Areolen.

437. Lecidea enteroleuca Arn. Jur. Sep. p. 164. - Ach. Syn. p. 19 p. p.

Die normale Form vom Panachaicon und vom Chelmos. Dic Pflanze vom Chelmos besitzt rorherrschend hogenförmige, selten lockenartige Pyenosporen und etwas verlängerte Sterigmen. Die f. atrosanguinea Hepp Eur. n. 252 von der Kyllene und dem Panachaicon. Die f. pungens Kr b. auf Krieselkalk von Olenos.

438. Lecidea alba Sch 1. Cat. p. 51.

Fin schr kleines Exemplar auf Pinus-Rinde vom Chelmos.

439. Lecidea atrobrunnea Schär. Spic. p. 14. - Ram. in DC. Fl. fr. 11, p. 367 sub Rhizocarp.

Myelohyphae J coerulescunt. Hypothecium incoloratum, luteolum v. rufo-fuscum. Sporae ellipticae $7-11.5 \mu$ lg., $4.7-5.5 \mu .1 \mathrm{t}$.

Zwei kleinere Exemplare auf Hornstein vom Chelmos, von welchen das eine durch das dunkle Hypothecium und die etwas grösseren Sporen der fumosa Hoffm. näher stelit.

440. Lecidea ecrustacea Arn. Verh. d. zool. bot. (ies. Wien 1874, p. 239. - Anzi exs. 903 sub Lec. polycarpa f. ecrust. 
Thallus depauperatus, myelohyphate J coerulcescunt. Apothecia salepe in rimis saxi sermata. 1lypa-

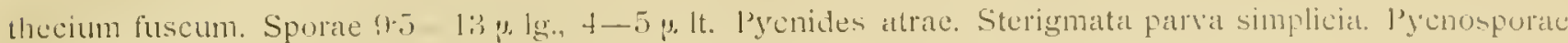

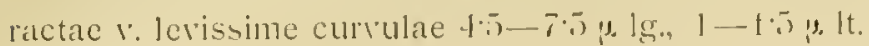

Auf Hornstein von Panachaicon.

141. Lecidea polycarpa Anzi exs. 17. 478. - Ann. Fil. 1871, p. 15̈2. - Noril. exs. 339.

Mycelohpphae J coeulescunt. Thallus albido-cincreus, habitu molliore, KH() primum lutescit, deinde sanguineo rubet. Hypothecium incolos $\because$. tandem luscidulum. Sporac non bene evolutac $11.5-14$ po lg.. $1.5-6 \mu .1 t$

Auf Kalkhornstein des J'anachaicon.

412. Lecidea meiospora Nyl. Sc. 225, F1. 1881, p. 5:34.

Thallus tenuis areolatu hydrate ferrico paullo tinctus madidus subvirescens. Apothecia minora mox

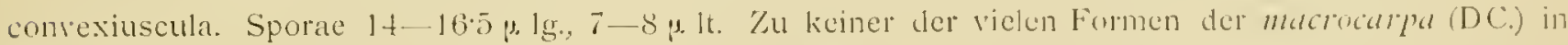

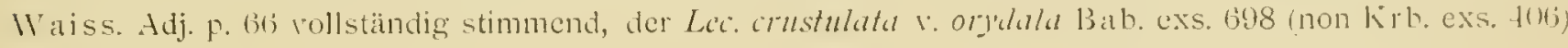
nahestehend.

Aul Hornstein rom Olenus.

44.3. Lecidea vitellinaria Ny. Bot. Not. 185: p. 17\%.

Die normale Pflanze aul Cal. rilcllima rom (helmos.

144. Lecidea Halácsyi Stnr.

Hyphac thallinae tenerac, incoluratae $i$. levissime tantum infuscatac areolam alienam percurrunt camque denecant et decolorant. Apothecia parva vix ad $0.4 m m$ diam.: erumpentia tandem adpressa crinvexiuscula $r$ convexa, immarginata, impure lusconigra, upaca, singula v. 3 - 4 congesta. Paraplyses fililormes, septatae, curvatulae et ramosac, laxiusculae, supra modice incrassatae.

Epithecium et excipulum obscure fuscum, atroviolaceun v. atroviride. Hymenium et hypothecium incoloratum, luteo-aurantiacum v. varie violaceofuscum. Asci elliptici $r$ clarati, membrana apicali incrassata ad $50 \mu . \lg ., 16-20 \mu .3 t$. Sporae octonae late ellipticae, rotundato-cylindricac 1 . elongatac passim leriter

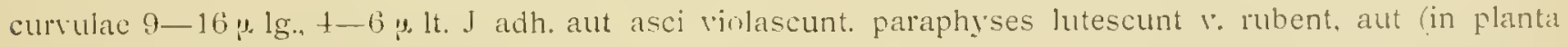
Epirot. conf. inf.) hymenium viulascit $r$ vinose rubet. KHO epithecium non mutatur, hynenium et hypothecium plus minus vinuse rubent. $\mathrm{HNO}_{3}$ colorem epithecii, praesertim atro-riride colorati, in violaceum sertit.

Dic Areolen ron Rhiwoc. gcograplicum von der Kyllene bewnhnend.

Die Areolen des WVirthes ändern fleckenweise oder reihenförmig ihre Farbe. indem sie zuerst grau ouler röthlichgrau und endlich braun werden, zugleich etwas einsinken und sich körnig zertheilen. Die Veränderung scheint bei ihrem Meiterschreiten zuerst die Markschichte zu treffen, deren Hyphen noch sehr lange ihre charakteristische Reaction gegen I beibehalten. Die Hyphen der Lec. Halácsyi sind in der Nähe des Hypotheciums als larbloses Fadennetz leicht zu verfolgen, dagegen im .larkgewebe des Wirthes nur nach Behandlung mit $\mathrm{KHO}$ und $\mathrm{HNO}_{3}$ als reiches, aber sehr zarthäutiges, kaum merkbar braunlich gefärbtes, toruböses Gewebe aufzufinden.

Der innere Bau der. Apothecien gleicht dem der Lec. supcrsparsa Nyl. Arn. exs. 1249 auffallend, su selur auch Wachsthumsweise und Tracht der Apothecien verschieden sind. Ausserdem fand ich bei supersparsa die Paraphyscnenden durch reichliche Gallerte verklebt, welche eine deutliche structurlose Deckschichte bildet, die der Lcc. Halicsyi lehlt. Die Sporen beider Arten sind öfter einander sehr ähnlich, doch bei Halacswi in ihrer Gestalt melu wechselnd, bei superspursa, soweit mir hekannt, immer gerade.

Wie oben angedeutet wurde, kommt Lec. Halácsiy auch auf Rliz. geogr. rom Peristeri in Epirus vor. Diese epirotische Pflanze ist es. Welch das dunklere Hypothecium und oft auch Hymenium und dic ctwels verschiedene Reaction gegen J zeigt.

Doch hängen diese Farben nur von einer Verfärbung des Pasma ab. Walche durchaus unconstant ist und die Jodreaction scheint mir nicht zu genügen, um eine besondere Fol m abzutrennen. 


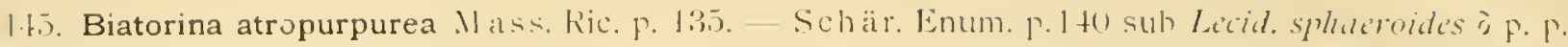
Ipothecia tenuiter marginata ad I $m m$ diam. disco passim papillato. Hyputhecium incoloratum 1 . luten-

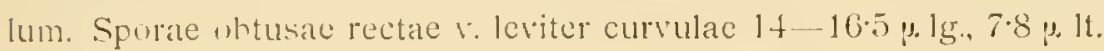

Ein wenig entwickeltes Exemplar auf Pinns-Rinde vom Chelmos.

146. Biatorina (Sect. Catillaria) athallina H Cpp. cxs. 409. - Arn. Jur. Sep. p. Iて̈.

Aul Kalkmergel rom l'anachaicon mit Hepp. exs. 499 vollständig ühereinstimmend.

4t7. Biatorina(Sect. Citillaria) pleiospora Stnt.

Thallus tenuis verrucoso-granulosus, cincren-ochraceus, lissuris lupis adpressus, reag. solitis non mutatus.

Apothecia parvis, primum urceolata, marginata, deinde atd $0.4 m m$ diam. deplanata, tandem convexiuscula, immarginata, atra. Paraphyses filiformes. laxae, supra septatae et cirpitulatae.

Epithccium apotheciorum juniorum obscure riride, hymenium et pars superior hypothecii fusci dilute coeruleo-viride. tandem epithecium obscure fusco-viride, hymenium decoloratum. Asci clavati membrana apicali incrassata ad $60 \mu .1$ g. $21 \%$. 1t. Sporae 16 in asco, ellipticae $v$. elongatae, obtusac rectac v. curvulac $7-12 \mu .1$ g., $4-5 \mu$. It. incoloratre, 1-septatae. Pycnides non vidi.

Die Farbenerainderung im hohen Hymenium während des Heranwachsens und dic grossen Asci mit je 16 Sporen dürten die Art genügend kennzeichnen

Der Thallus in kleinen Gruben und Ritzen des Gesteines ist neist dürtig, nur an ciner Stelle ctwas mehr entwickelt.

Auf Hornstein des Panachaicon.

448. Buellia verruculosa Th. Fr. Sc. p. 600. - Borr. in Engl. Bot. 1811, t. 2317.

Areola convexiusculae, stramineate $r$. decoloratae. Hypothallus obsoletus. Apothecia tandem cmersa. Die Flechte kommt der R. jugomm Arm. nahe

Auf der opalartigen liesclausscheidung rom Olenos mehrere, abor wenig entwickelte Thallusfleckichen.

449. Buellia (Sect. Diplolomma) epipolium Th. Fr. Sc. p. 600. - Arn. Jur. Scp. p. 195 sub Diplot. Ach. Prodr. p. 58 sub Lichene.

Auf lialk mit Hornstein vom Panachajeon.

Daselbst auch die f. margaritaced Somm r. I.ap p. F. 148 und murormm Mass. Ric. p. 98.

150. Rhizocarpon geographicum DC. Fil. Fr. II, p. 365. Linn. Spec. Fl. I. p. 1063 sub Lichenc.

Häufig die normale Pflanze auf Kicselkalk von der liyllene und dem Panachaicon und aul Sandstein rom Chelmos.

451. Rhizocarpon obscuratum kỉb. Syst. p. 216. - Ach. Lnir. p. 156 sub. Lec. petrata obscur.

Ein klcines Exemplar vom Olcnos mit Placospora rimosicolu.

452. Rhizocarpon distinctum Th. Fr. Sc. P. 625. - Sy'n. Lec. alboulsa ambigma lirplh.

Thallus cinereus, fuscocinereus v. p. m. p. plumbeo obscuratus, myelohyphae J violascunt v. pas. sim violascunt. Sporae ellipticae v. varie deformatac $28-33 \mu .1 \mathrm{~g}, 15-17.5 \mu .1 t$. diu incoloratae, tandem dilute viridescentes et fuscescentes, fauce septatac. Epithecium KHO magis violascit, $\mathrm{HNO}_{3}$ roseo violascit.

Hläulig auf lialk mit Quarz vom Panachaicun.

453. Rhizocarpon concentricum 'Th. Fr. Sic. p. 627. - Jar. in Trans. Linn. Soc. 2. p. 284.

Nach dem Originalexemplar im Herb. Eggerth (Univers. Wien) genau dieselbe Flechte, welche Arn. Flor. 1887, p. 151 rom Mt. Deca aul Corfu anfülurt.

Auf kalk mit Hornstein rom Panachaicon.

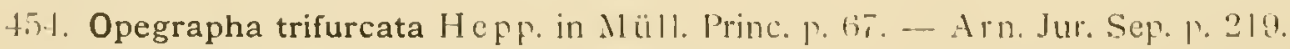

Nur einige Apothecion auf Kalk l'om P'anachaicon. 
455. Melaspilea oleae Stn 1 .

Thallus epiphloeodes tenuissimus, ohscurus constat ex hyphis fuscis subrotundato cellulosis. (innidiat palmellea fortuito tantum instrata. Apothecia Melasp. mearalynate A 11. li1. 1881, p. 205. - Ach. Univ. p. 2-14 similiat.

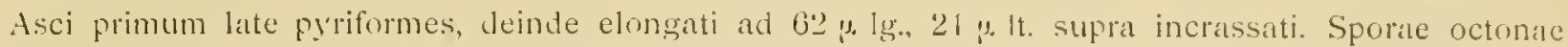
ad 19 y. $\lg ., 7-95$ j. 1t. incolores 1 -septatae cellula altera subrotunda. Paraphyses crassae, rotundato-cellulosae, supra infuscatac epithecium fuscum cellulosum formant. Hypothecium quoque cellulis rotundis instıuctum plus mimus luteofuscescens.

In der äusseren Form, der Gruppirung und Farbe der Apothecien und ehenso in Form und Grösse der Sporen gleicht die Pllanze der Mel. Mlgg alyar. Dagegen ist sie durch die rundlichen, abgeschnürten Zellen des Hypotheciums und besonders der Paraphysen, die im Epithecium eine Dicke von j-6 chen, von allen mir bekannten Aiten del Gattung Mclaspilea hodatend verschieden, auch von . M. opgraphoide's Bagl., welche ebenfalls aur Ölbaumrinde wäichst.

Auf glatten Ölbammrinden bei Patras.

456. Arthonia vagans var. Kürlerti Alm 4. Artl. p. 51.

Auf Kalk von lialarryta.

Hyphae thallinae J coerulescunt. Pycnosporac breviter hacillares. 3- 4.8 is lg., $0.8-1$ is it.

457. Placidium compactum Mass. Misc. p. 32. - Arn. e.s. n. 267.

Asci primum elliptici subpedicellati, deinde elongati. Sporae $8-13$ u. $1 \mathrm{~g} . .7$ - 9 p. It. late ellipticae v. lele subrotundae. Die Sporen sind allerdings etwas kleiner und oft breiter als in Arn. 267 , aber im Ganzen entspricht die Flechte genügend.

Auf kilk des Panachaicon.

458. Placidium rufescens var. hiperiforme Mass. Sched. p. 111.

Sporae elongato ellipticae $16-17$ ig., 7 i. It.

Nur einige Areolen auf lialk von kalavryta.

459. Dermatocarpon pusillum Arn. Jur. Sep. p. 239. - Hed11. Stirp. Crypt. 1789. p. 36 sub Endoc. Gonidia hymenialia rotunda $v$. subquadrata $4-5.5 \%$ diam.

Auf Erde bei Patras.

460. Verrucaria (Sect. Lilloicial) trachytica Hazsl. in Rab. exs. suh Endoc.

Aul kalkconglomerat rou kalavryta.

461. Verrucaria (Sect. Lithoicea) nigrescens Nyl. Pyren. p. 23. - Pers. Ust. Ann. 1795. p. 36 F. F. Auf lialk von Patras und lialaryta.

462. Verrucaria (Sect. Lithoicea) controversa Mass. Ric. p. 17\%, Fig. 358 et exs, n. $21,195$.

Sporac forma varia sed praesertim late ellipticae $23 .-26 \mu . \lg ., 12-15 \mu .1 t$. Gelatina hymenea et asci J rubent.

Auf Kalk bei Patras.

463. Verrucaria (Sect. Lilhoicea) cataleptoides Nyl. Prodr. p. 18:2. - Syn.: Vert. calalepla sichäir. Enum. p. 211 p. p.

Sporac ellipticae 18-23\% lg., 9-12 It. Der diurftige Thallus in die Gesteinsritzen gedrückt. Die Pllanze gleicht $Z w$. exs. n. 150.

Auf Hornstein vom Olenos.

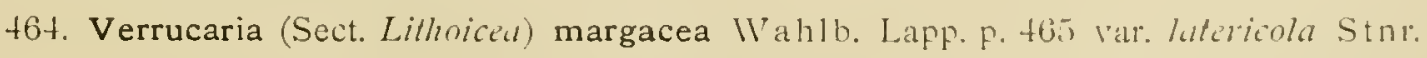

Thallus tenuis effusus cinerco-argillaceus subfarinosus, circa perithecia rimoso-areolatus, areolae tandem ad marginem a latere páullo solutae. Perithecia mediocria dimidiatim denudata, atra. Sporae octonae, late ellipticae v. subrotundae $19--31$ \%. Ig., $1+-18$ \%. 1t. Hymenium .J primum coerulescit. deinde praesertim gelatina vinose decoloratur. 
Von der normalen margacea durch den Thallus und theilweise die Perithecien verschieden.

Auf einem Stück Ziegelstein hei Patras.

465. Verrucaria (Sect. Amplintidium) Veronensis krh. Par. p. 361. Mass. Ric. p. 173 et lig. 3.t3 suh Amplior:

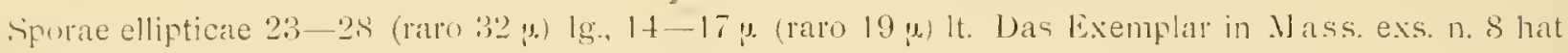
etwas kleinere, mehr eingesenkte Perithecien, dagegen entsprechen Exemplare ron den wberbairischen Alpen aus dem Herb. Eggerth (Univ. Wien) vollständig.

Auf kalk von Patras.

466. Verrucaria (Sect. Amphorid.) dolomitica Krb. Par. p. 362. - Mass. Sym. p. 80 sub Amphurid.

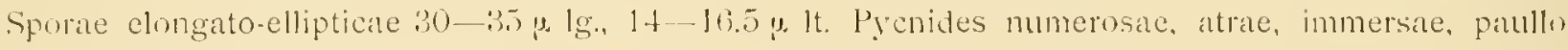
emergentes. Arthrosterigmata cellulis apicalibus tantum fertilibus. Pycnusporate breviter bacillares., rectile v. leviter curvulae $35-5-7 . \lg .05 \%$. It.

Auf Kalk von Kalarytat und dem Panachaicon.

467. Verrucaria (Sect. Amphoridimm) tetanocarpa Stmi.

Thallus in calce maculam albilam formans.

Perithectia immersa, integre nigra, apice tantum convexiusculo et pertuso emersa, cylindrica 1 . subprismatica at $0 .+13 m$ dians. et $0.5 \mathrm{~mm}$ alta.

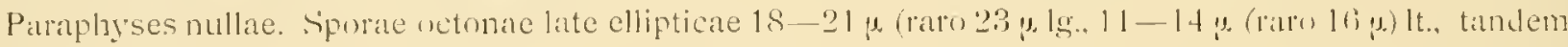
luscidulae.

$\mathrm{J}$ coerulescit $\mathrm{v}$. gelatina vinose ruhet

Auf Kalk vom Panachaicon.

Die kleinen, bald leicht braunlichen. Sporen gleichen denen der l'orr. Kïrbri; dureh die Form der Perithecien aber scheint mir die Art genügend gekennzeichnet.

468. Verrucaria maculiformis Kiplh. Fl. 1858 . p. 303.

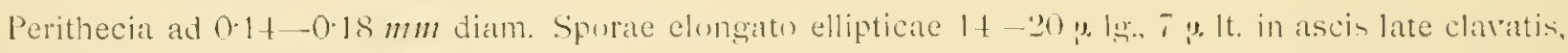

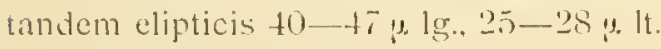

Auf Kalli vom Panachaicon.

160. Verrucaria calciseda 1) ( : 1.1. Ir. 1, 180, , p. :317

1)ie normale Pllanze aul Kalk rom Panachaicon.

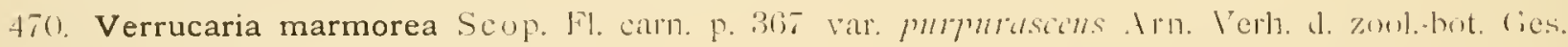
Wien 18i2, p. 307. - Hoffm. Pl. Lich. p. 7 t.

Auf Kalk vom Panachaicon.

471. Arthopyrenia Persoonii Mass. Symm. p. 410.

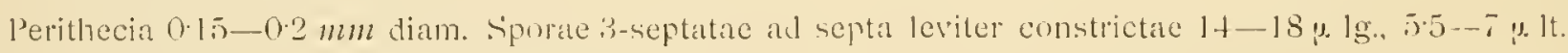
in ascis elongatis. supra attenuatis ad $54 \mu, \lg , 16 \mu, 1 \mathrm{t}$.

Aul glatter Ölbaumrinde von Patras.

472. Pharcidia congesta Krb. Pill. p. 470.

Perithecia minima vix ad $(1)$ mm diam. luscal v rufofusca. Sporae octonae graciliter subbacillares usque

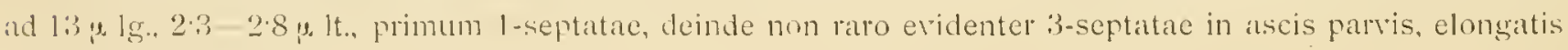
$32-38 \mu .1 \mathrm{~g} ., 9-12 ; 1 \mathrm{t}$.

Aul Lecam. anguhlusa rom Olenos, das Hymenium des Wirthes bewohnend und bräunend. Die Perithecien nehmen den Disculs endlich filst vollstïndig in Besitz.

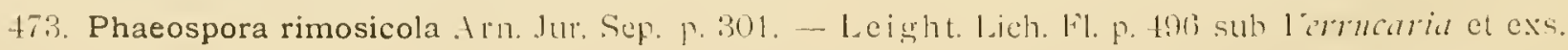
ก. $25 \% 3$. 
Sporae octonae, elongato-ohlongate i3-septatae ad septa levited constrictac, incolores, tandem fuscate

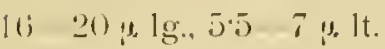

Aul dem Thallus ron Rhizocarp. obscuratmm vom Olenos.

473. Tichothecium pygmaeum krh. Par. p. 467. - lir. Lich. Spitzb. 51 suh Endococco.

Die normale Pllan\%e auf dem Thallus der Lace. declinasens rom Chemos und der Lecam. calíaral rar.

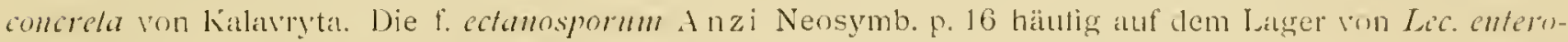
lanca, Calopl. intercedens, Lecan. Agardhiana und Rinod. Bischofiii iom Panachaicon.

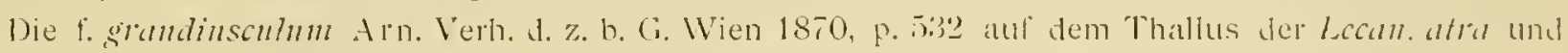
Léci. caliarà var. virilescens rom Panachaicun.

Perithecia gregatim congesta ad $(1.25)$ mm diam.

47. Tichothecium erraticum Mass. Symm. p. 94.

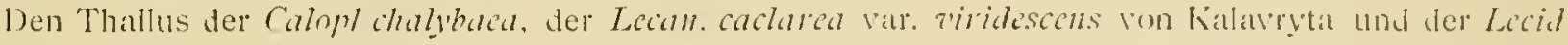
cmlimlinia ion chemos berrohnend.

Asci mo. elongati, magni, tandem ad 70 y. lg., $18-2: 3$ y. It. Sporae numerosae magnituline varia, medin non constrictale $6-12 \mu 1 \mathrm{~g} ., 4-i \mu .1 t$.

475. Tichothecium macrosporum Arn. Verh. d z. b. (j. Wien 1868, q. 900.

iporac octonae, elongatae, apice altero r. utroque attenuatae, medio sapius leviter cunstrictac 18 usque ad $23 \% \lg .7-9$ \%. it.

Auf dem Thallus der Lci: chlerolenca lom P'anachaicon.

476. Tichothecium calcaricolum Arn. Verh. 4. \%. b. G. Wien 1873, p. 521. - Mudd. Man. F. :30ti suln Vicrothelia.

Perithecia ad $0.25 \mathrm{~mm}$ diam. Sporae ellipticae, medio leviter constrictae, dilute fuscae, $12-15 \mu .1 \mathrm{~g}$. $7 \%$. It.

Auf dem Thallus der Lecan. calcarea valr. concrela rom Panachaicon.

47. Müllerella dilatata Stnr.

Perithecia primum subglobosa, thallo alieno immersal ad $0.35 \mathrm{~mm}$ diam., integre rufo-fusca, circa porum valde incrassata nigro-fusca, deinde dilatata pseudodiscum atrum, thallum altitudine vix superantem, formantia. Paraphyses filiformes, ramosae et septatae saepe ad $0 \cdot 4 \mu 1 \mathrm{t}$. tandem supra infuscatae. Asci primum late elliptici stipite brevi instructi ad $54 \mu$. Ig., $33 \mu$ lt., deinde elongati ad $100 \mu .15 ., 19 \mu .1 t .$, memblana apicali

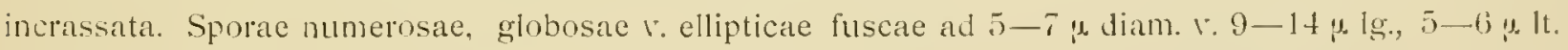

Auf dem Thallus der Lecan. trachytica rom Panachaicon und ron der Kýllene.

Von den Perithecien aus sieht man, wie die Hyphen das Gewehe des Wirthes durchwachsen, ohne es zu verandern. Die Art ist durch die grossen Perithecien ausgezeichnet, deren Purus sich endlich so erWeitert, dass sie kreiselförmig werden, oflenfrüchtig scheinen und in liadialschnitt den Apothecien mancher Calicien gleichen. 


\section{Verzeichniss der Gattungen.}

Dic erste 7ahl bezieht sich auf die betreffende Seite des Separatabdruckes, die zweite (in Klammern hefindliche) auf die fortlatufende Paginisung des Bandes der Denkschriften der kais. Akademie.

\begin{tabular}{|c|c|c|c|}
\hline Acarospura.... 40 [526] & Calamintha $\ldots .30[514]$ & Farsetia ...... $11[495]$ & Lecaniar....... to [52t \\
\hline Heer . . . . . . . 16 16[500] & Caluplaca ..... .38 [522] & Ferula.......21 [505] & Lecanora ..... to $\mid 524]$ \\
\hline$\therefore+4[508]$ & Campanula ...20 [510] & Ferulago...... 221 [505] & Lecidea \\
\hline Aclonis........ 9 [493] & Carduus . . . . 25 [509] & Festuca ...... .34| & Leontici....... 9 $9[493$ \\
\hline Aethionema ... $13[497]$ & rarex ........34 [518] & Filago ........24 [508] & Liontodon . . . . .26 [5]101 \\
\hline Aira $\ldots . . . .34[518]$ & $\ldots \ldots 22[506]$ & Freyeral . . . . ..22 [506] & Lepidium . . . . 1:3 [497] \\
\hline Ajugat .........28 [512] & Celsicl $\ldots \ldots \ldots 27[511]$ & Fritillaria.....33[517] & Leptodon . . . . 37 [5:211 \\
\hline Alkanur . . . . 26 [510] & Centaturat......25 [509] & Frullaniu ... . .37 [ & Leptotrichum ...35 \\
\hline Alliaria . . . . 10 [494] & Centrunthus. ...23 [507] & Fumaria .....10 & Lencodon . . . .37 [521] \\
\hline $.4 l l i m m \quad \ldots \ldots . .33[517]$ & Ceplualanthror ...32 [516] & Funaria .......36 & Ligustrum ....26 \\
\hline Alsine . . . . . $15[499]$ & Cerastium..... I5 [499] & Gagea ..........33 & Linnm,.......10 \\
\hline Allhaea ........ $16[500]$ & Ceratoceplualus. . 9 [493] & Galactites.....24 & Lolium ........35 \\
\hline$A / y ' s s u m \ldots . .12[496]$ & Chamaepouce ...24 [508] & Galium ........23 & Lonicira ......2: \\
\hline Anсmonс. . . . . 9 $9[493]$ & Clematis.......9 9 [493] & Gaudinia ......35 & Lusula ........34 \\
\hline Anthemis .....24 [508] & Collema .... & Genista .......17 & Lysimachia .....30 \\
\hline Anthericum ... .33 [517] & Coninm ........22 [506] & Geranimm.....16 & Lythrum .....20 \\
\hline Arabis......... 10[494] & Coryclalis ......10 [494] & Globularia ....30 & Madotheca \\
\hline Aremonia .... & Cratalegus . . . 19 [503] & Grimmia . . & Malabata. . . .21 [.50s] \\
\hline Arencuria . . . . 15 [494] & Cropis . . . . . . 25 [509] & Givalolectriat & Malcolmia $\ldots \ldots 10[494]$ \\
\hline Armeria .......30 [514] & Crocus.........33 [517] & phila. ... 15 & Malia...... \\
\hline Arthonia.......47 [531] & Crupina .... & olobium 17 & Matricarial ...2415081 \\
\hline Arthoplremia . . 48 [532] & Cyrarara .....24 & Helianth & Melaspila . \\
\hline Arum .........34 [518] & Dactylis........34 & Herniaria.....20 [504] & Melica. .......34 $\lfloor 518 \mid$ \\
\hline Asperulat. & Delphinimu $\ldots .99$ & Hicracium ... 25 & Melitotus.. \\
\hline Astrugalus & Dermatocarpon . 17 & $s \ldots 18$ & Melittis. . . . . 29 2513$\rfloor$ \\
\hline Atripler & Diantlius.... . . $15[499]$ & Homalothecium .37 & . . . .23 $[50 \overline{5}]$ \\
\hline Aubrietia ......11 [495] & Didy'modon . . . . 35 & icnm . . . 16 & Museari . . . .33 [517] \\
\hline$\ldots 34[522]$ & Digitalis ......27 [511] & Hypmum . . & Miillerella... \\
\hline Ballota ........30 & Diploschistes. . & Hypochaeris. & Myosotis . \\
\hline $\begin{array}{l}\text { Barbula ... } \\
\text { Bellis..... }\end{array}$ & $\begin{array}{l}\text { Dorycuiam .....17[501] } \\
\text { Drata........11[495] }\end{array}$ & Iris .... & Nasturtium... \\
\hline Berteroa ......11[495] & Drypis. . . . . . 15 [499] & Isothecium & Oenantlic \\
\hline Biatorina . . & Ephedra .......35 [519] & | & Onobrichis . . . 18 $\left[50^{\circ}\right]$ \\
\hline Bilimbiat . . . . . 43 [527] & Equisetum......35 [519] & & $.17[501]$ \\
\hline Bonanmia .......1 [505] & Eryugium . . . . .222 [506] & & Onoporilon. \\
\hline Bromus.......34[518] & Erysimum $\ldots .10[494]$ & 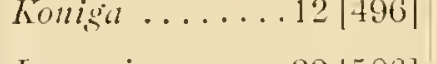 & Onosmat \\
\hline$\ldots \ldots 36[520]$ & Encladium .....35 [519] & Lagoccia. & Opegraplat... \\
\hline Buclliu .......46 [530] & Euphorlia ....31 [515] & Lagoseris & Ophris . . . . . 32 [516] \\
\hline Bulbocastatnum .22 [506] & $.2+[.500]$ & Laminn & Opopana... \\
\hline & & lathiverss & rchis \\
\hline
\end{tabular}




\begin{tabular}{|c|c|c|c|}
\hline 11111 & -1 & Scitc & (ni \\
\hline$\cdots \cdot{ }^{3}>{ }_{3}$ & Primula & Scilla........33[517] & Trichustommm ... \\
\hline 02$]$ & $\ldots 29$ & uthus. ...20 & $m m$. \\
\hline$m \ldots .36$ & Prumus .......1s & $m m \ldots 3 t$ & Tulipu.... \\
\hline .31 & lus . . 293 & $\cdots$ & .1 \\
\hline .38 [522] & $P u t$ & Sculcllarial . ..29 & l'mh \\
\hline$\ldots 38$ [522] & On- & Sednm.... & ... \\
\hline$\ldots \ldots+3[527]$ & Qnet & .24 & $L^{\circ}$ \\
\hline $14 \ldots+8$ [532] & & Sesleria... & \\
\hline ........+8|532| & 21] & $.14\lfloor+98 \mid$ & ma \\
\hline totis .....36|520] & ic $>$ & Sisymbrinm & Valcriuncllu \\
\hline$\ldots . .30[514]$ & & Smbrninm . & Verbasiam \\
\hline [4......38|522] & & Specularia & Vero \\
\hline $.26[510]$ & 6241 & Stacly's .... & .47 \\
\hline $.30[514]$ & Rosa & Sternbergia. & Vicis \\
\hline $.35[519]$ & Rumex & 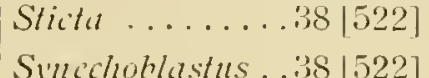 & l'iola. \\
\hline $.47[53$ & & .381 & $X_{a l}$ \\
\hline 311 & 12] & $\ldots 20 \mid 50$ & . \\
\hline 18] & iaponaria & $m \ldots \ldots 28$ [512] & \\
\hline .26 & aponaric & $\ldots 49[533]$ & Zigodon. \\
\hline 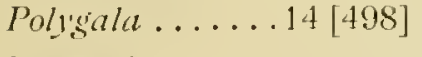 & $\begin{array}{l}05] \\
06]\end{array}$ & i...... & \\
\hline Hentilla & 06] & Thy'mus. . & \\
\hline
\end{tabular}








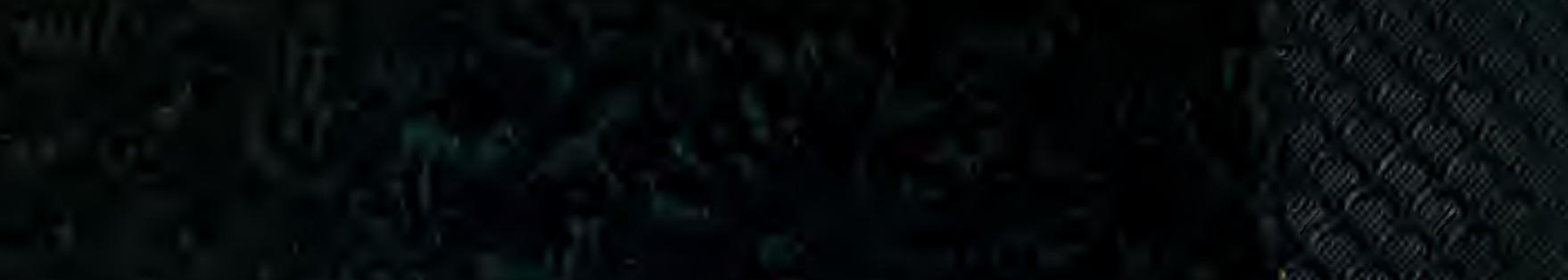

18\%ors

Ton 